\title{
Auditory associative learning and its neural correlates in the auditory midbrain
}

\author{
Dissertation \\ for the award of the degree \\ "Doctor rerum naturalium" \\ of the Georg-August-Universität Göttingen \\ within the IMPRS Neuroscience program \\ of the Georg-August University School of Science (GAUSS)
}

submitted by

Chi Chen

born in

Hunan, China

Göttingen 2018 



\section{Thesis Committee}

\section{Dr. Livia de Hoz (Supervisor)}

Department of Neurogenetics, Max Planck Institute for Experimental Medicine

Neuroscience Research Center, Charité - Universitätsmedizin Berlin

\section{Prof. Dr. Robert Gütig (Reviewer)}

Department of Neurogenetics, Max Planck Institute for Experimental Medicine

Mathematical Modeling of Neural Learning, Charité - Universitätsmedizin Berlin and the Berlin Institute of Health (BIH)

Prof. Dr. Tim Gollisch (Reviewer)

Sensory Processing in the Retina, University Medical Center Göttingen

\section{Prof. Dr. Alexander Gail}

Sensorimotor Neuroscience and Neuroprosthetics, German Primate Center

\section{Members of the Examination Board}

\section{Prof. Dr. Tobias Moser}

InnerEarLab, Institute for Auditory Neuroscience, University Medical Center Göttingen Synaptic Nanophysiology, Max Planck Institute for Biophysical Chemistry Auditory Neuroscience, German Primate Center

Auditory Neuroscience, Max Planck Institute for Experimental Medicine

Prof. Dr. Julia Fischer

Cognitive Ethology Lab, German Primate Center

Dr. Camin Dean

Trans-synaptic Signaling, European Neuroscience Institute Göttingen

Date of oral examination: 21 th January 2019 


\section{Table of Contents}

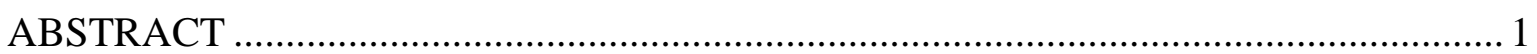

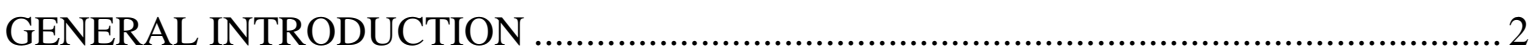

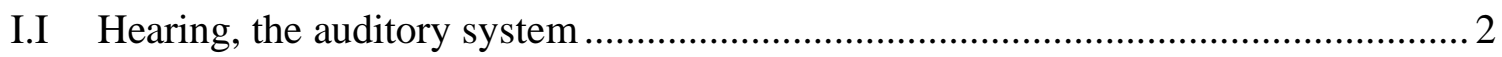

I.II Learning-induced plasticity in the auditory system........................................... 2

I.III Task-related modulation in the auditory system.............................................. 5

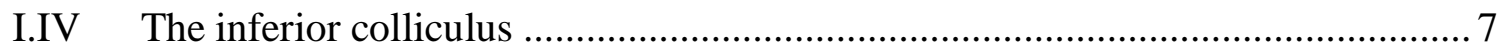

I.IV.I The hub of auditory integration in the midbrain .......................................... 8

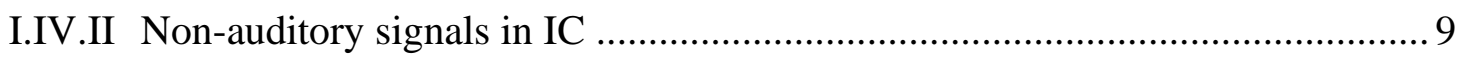

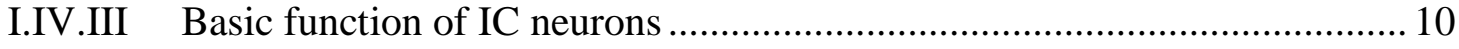

I.V The general question and the particular questions we want to address .................. 10

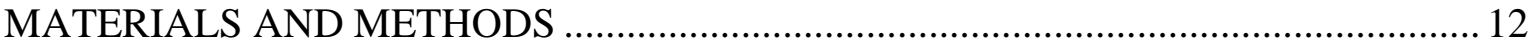

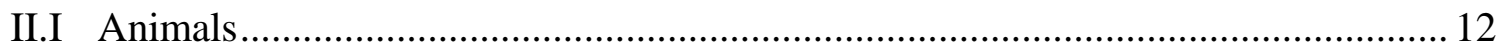

II.II Memory-based discrimination learning and generalization in the Audiobox ..... 12

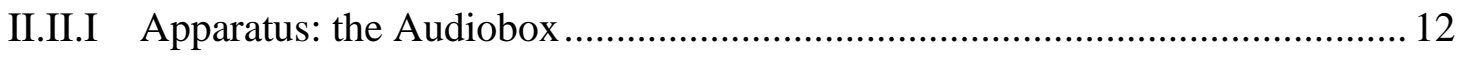

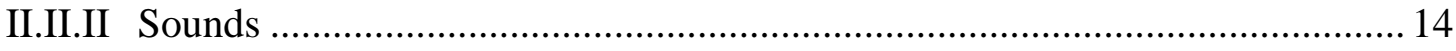

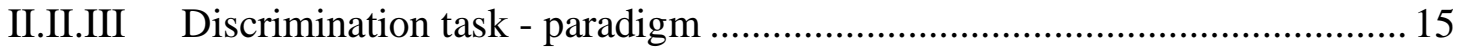

II.II.IV Generalization gradients for tone frequency ......................................... 15

II.II.V Multidimensional generalization measurement ........................................... 16

II.II.VI Analysis of performance in the Audiobox ................................................ 16

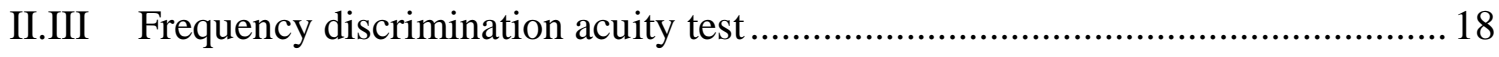

II.IV Electrophysiological recording with chronic implanted tetrode microdrives .....21

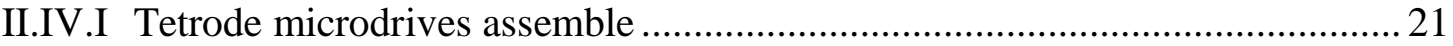

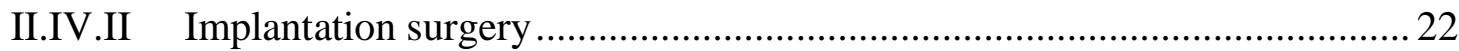

II.IV.III Discrimination training in the Soundterrace .............................................. 24

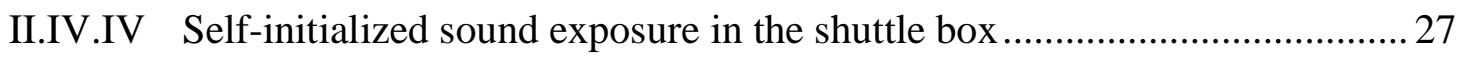

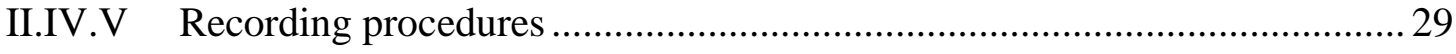

II.IV.VI Analysis of electrophysiological recordings............................................. 30

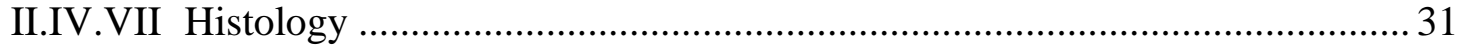

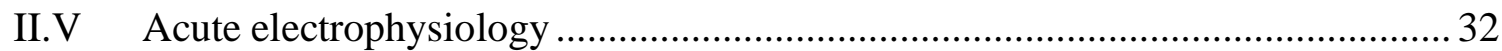




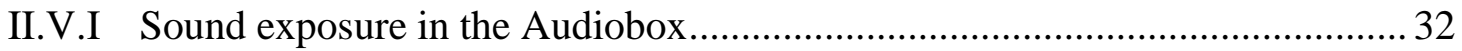

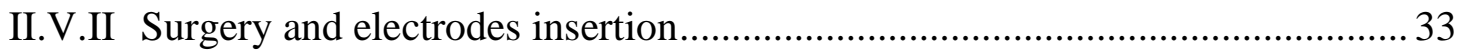

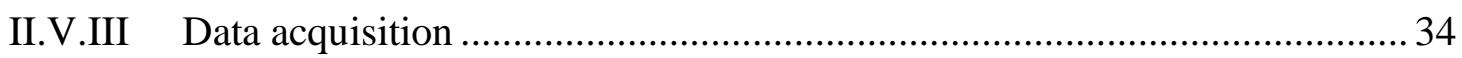

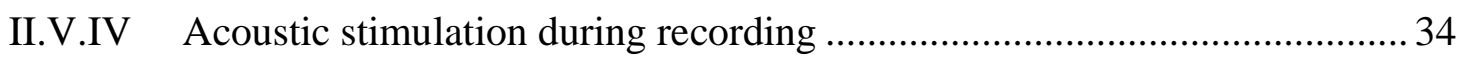

II.V.V Analysis of electrophysiological Recordings .............................................. 34

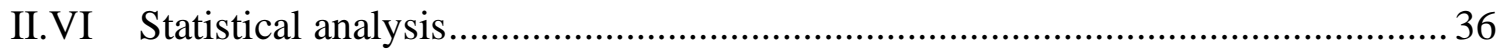

Chapter 1 Wide sensory filters underlie performance in memory-based discrimination

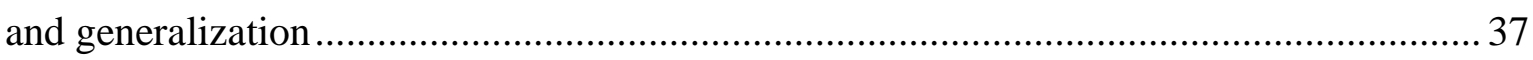

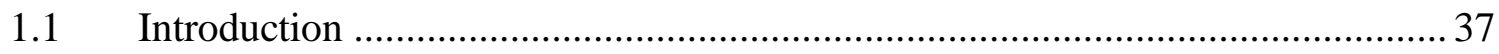

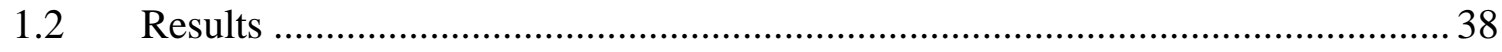

1.2.1 Mice associated acoustic stimuli with different behavioral outcomes and generalized the learnt association to novel stimuli.

1.2.2 Discrimination task performance deteriorated as the safe-to-conditioned $\Delta \mathrm{F}$

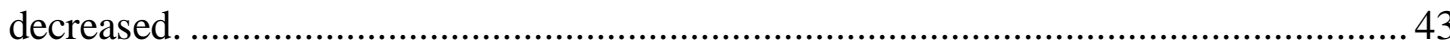

1.2.3 Shifts in the psychometric threshold with decrease in $\Delta \mathrm{Fs}$..........................46

1.2.4 Neuroligin 2 knockout mice showed impaired discrimination performance but normal generalization gradient.

1.2.5 The direction of conditioning along the frequency axis influences discrimination learning but not generalization

1.2.6 Previous experience with the task did not facilitate discrimination but shifted generalization gradients

1.2.7 Discrimination acuity was increased around the conditioned tone after Audiobox learning in a $\Delta \mathrm{F}$-specific manner. ............................................................ 57

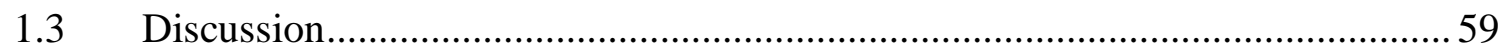

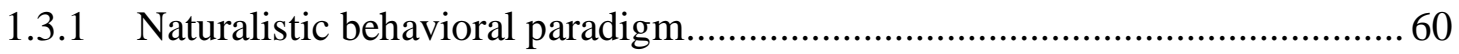

1.3.2 The physiological constrain is prioritized in perception ................................61

1.3.3 Valence has a secondary modulation influence on generalization ..................62

1.3.4 Discrimination performance and generalization are differentially modulated by

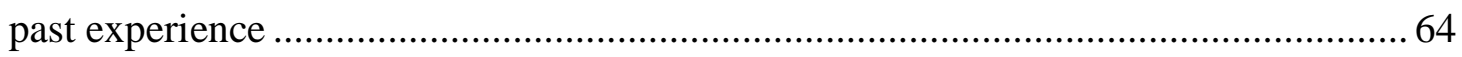

1.3.5 The dissociation between perceptual discrimination and generalization ........64

Chapter 2 Bidimensional generalization reveals hierarchical organization of acoustic dimensional processing

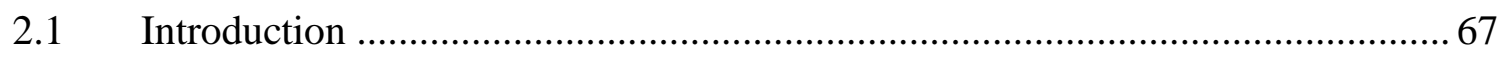

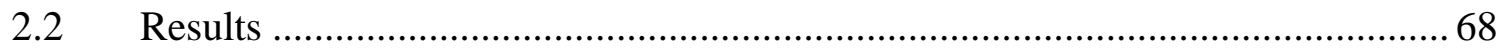

2.2.1 Mice generalized mainly along the dimension of frequency range, but not FM

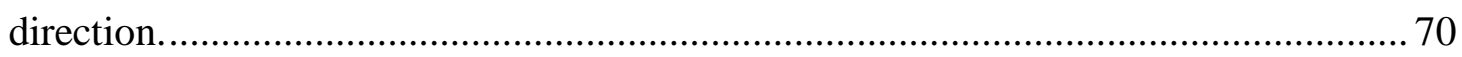


2.2.2 Hierarchical organization of FMs perception in mice .72

2.2.3 Mice had the flexibility to be trained to do unidimensional categorization. ... 74

2.2.4 When the discrimination task was along the non-preferred dimension, learning becomes more localized. .76

2.2.5 Hierarchical organization of 'envelope' perception in mice 77

2.2.6 The combination of sound dimensions used in the task influenced task performance.

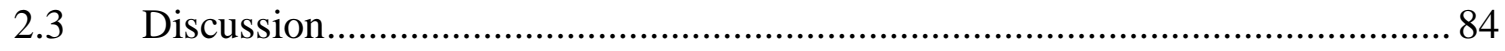

2.3.1 Hierarchical organization of the low-level acoustic dimension processing .... 85

2.3.2 Neurophysiological consideration of dimension perception .86

2.3.3 Methodology considerations. .87

Chapter 3 Auditory learning modulates neuronal activity in the Inferior Colliculus... 89

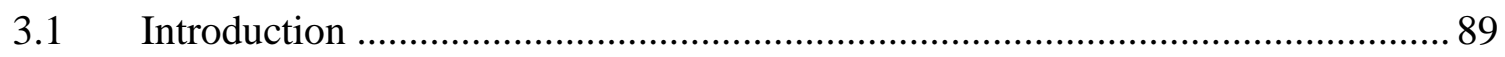

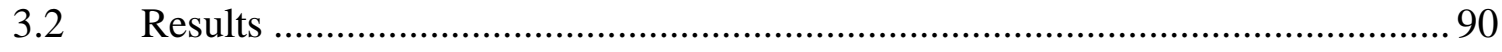

3.2.1 Pure-tone frequency discrimination task in the Soundterrace ........................ 90

3.2.2 Task engagement increases spontaneous activity in IC.................................92

3.2.3 Task engagement selectively suppressed the sound-evoked responses to the conditioned tone

3.2.4 Global increase in gain and signal-to-noise ratio after a discrimination task.. 97

3.3 Discussion. 100

3.3.1 What is the cause of the effect associated with task engagement?. 101

3.3.2 Mechanisms of modulation induced by task engagement ............................ 102

3.3.3 The persistent plasticity in periods immediately following behavior............ 103

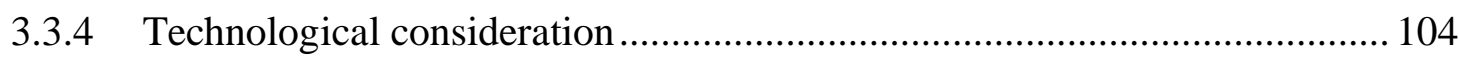

Chapter 4 Acoustic consequence of movement is the key factor for IC plasticity

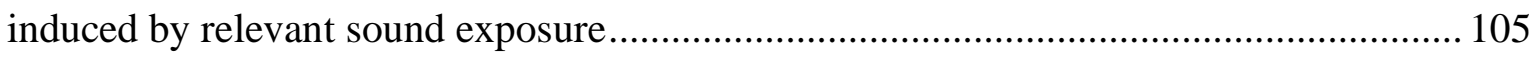

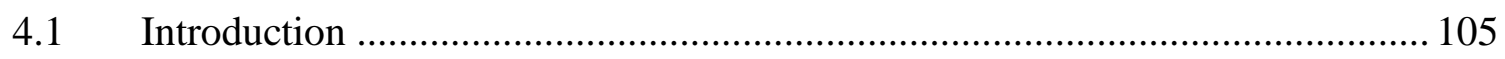

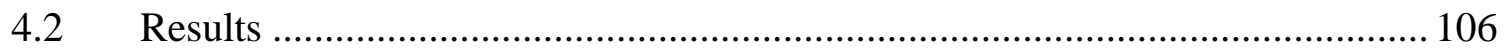

4.2.1 Sound exposure in the corner elicits IC plasticity independent of water association .....

4.2.2 Exposure to unpredictable sound in the corner does not elicit plasticity in the inferior colliculus

4.2.3 Sound exposure as a consequence of movement is the key factor for IC plasticity induced by sound exposure. 
4.2.4 Sound exposure does not change sound-evoked or spontaneous activity in IC of freely behaving mouse

4.2.5 Movement that initiates sound exposure suppresses ongoing local field

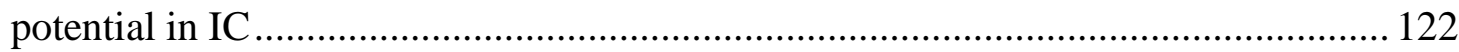

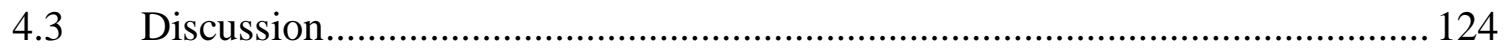

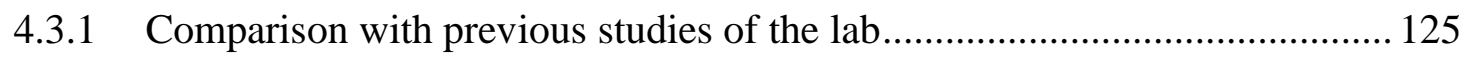

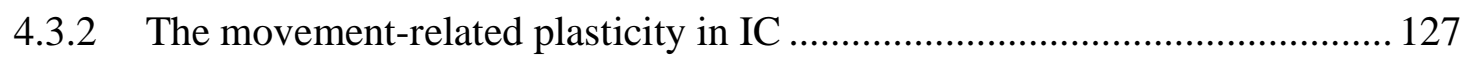

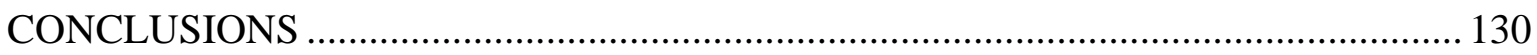

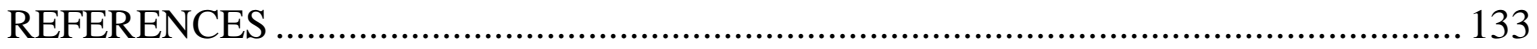

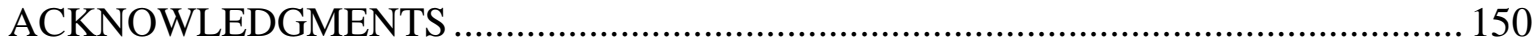

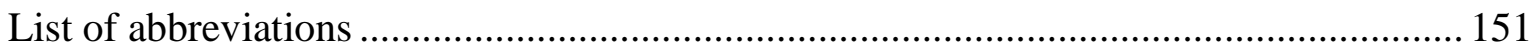

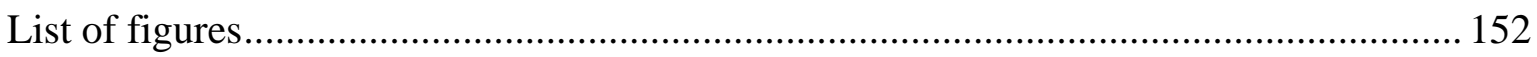

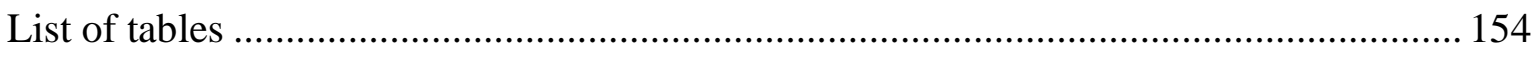

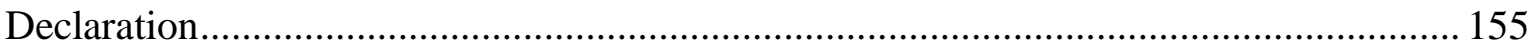




\section{ABSTRACT}

Interpreting the meaning of environmental stimuli to generate optimal behavioral responses is essential for survival. Simply sensing a sound, without accessing prior knowledge in the brain, will not benefit behavior. How sensation and memory interact to form behavior is one of the fundamental questions in the field of neuroscience. In this thesis, I have addressed this question from two perspectives: I investigated the behavioral outcome of this interaction using discrimination, and the circuit underlying this interaction using electrophysiological recordings in the behaving mouse.

Behaviorally, we found that the physical difference between to-be-discriminated sounds, had a constraining effect on discrimination. This effect occurred even though physical differences were significantly larger than reported discrimination limens, thus reflecting a high overlap between the memory traces of the relevant stimuli. The results suggest a strong role of pre-wired tonotopic organization and the involvement of peripheral stations with wider tuning (Ehret and Merzenich, 1985; Taberner and Liberman, 2005). To further understand the influence of sensation on behavior, we tested the interaction between sound features with generalization. Using sounds that differed in two dimensions, we found that bi-dimensional generalization can be either biased towards a single dimension or an integration of both. Whether it was one or the other depended on the two dimensions used.

As the first convergence station in the auditory system (Casseday et al., 2002), the role of the inferior colliculus in encoding behavioral relevant information is not well understood. Recording from freely behaving mouse, we found task engagement modulated neural activity in the IC in a stimulus-specific manner. Our lab found previously that relevant sound exposure induced enhancement in neural activity and shifts in tonal representation in the IC (Cruces-Solís et al., 2018). As a continuation, we found that movement-sound association is essential for this plasticity. Furthermore, recording in freely behaving mice also found that this association modulated the ongoing LFP in the IC, suggesting a new role of IC in filtering movement-related acoustic stimuli.

To conclude, our results support the view that the IC is not simply an auditory structure that relays auditory information into the cortex, but plays important role in interpreting the meaning of the sound. The new role of IC in encoding movement-related information suggests that the filtering function of the auditory system starts already in subcortical stages of the auditory pathway. 


\section{GENERAL INTRODUCTION}

\section{I.I Hearing, the auditory system}

To survive, an organism must have the ability to sense and interpret the external environment to form optimal behavioral responses. Sensation relies on the sensory systems to transduce information of the external environment into neural signals that can be further processed in the brain. In the auditory system, how a sound stimulus is sensed has been well studied. The mechanical vibration, a sound, enters the ear canal causing vibration of the auditory ossicles which, in turn, cause a vibration of the liquid that fills the cochlea. Hair cells in the cochlea transduce the mechanical information into electrical signals which travel along the auditory nerve to the brain. Before reaching the auditory cortex, the reencoded electrical information passes through multiple intermediate auditory stations, including the midbrain and thalamus.

The auditory system is remarkable in that it senses continuously and detects stimuli when they are far away, which makes it an optimal warning system. However, how the sensation of auditory information is interpreted to generate meaningful perception is not well known. Interpretation should require prior knowledge, which can be acquired through associative learning where two elements are linked in the brain (Wasserman and Miller, 1997), such as when the sound of a bell is linked to food.

Traditionally, the auditory system was viewed as a purely sensory organ that provides raw information about the environment, and whose response characteristics remained unchanged except during the so-called "critical" period before adulthood (Hensch, 2005). However, accumulating evidence shows that associative learning can induce stimulispecific changes in the sensory system. Plasticity at the sensory system indicates an interaction between sensation and learning, which further suggests that the role of auditory system is not only to pass sensory information but to filter meaningful information.

\section{I.II Learning-induced plasticity in the auditory system}

The effect of learning on plasticity in the auditory system was first described in 1956 (Galambos et al., 1956). Response in both cochlear nuclear and auditory cortex was increased after pairing an electrical shock with clicks (conditioned stimuli, CS). In the auditory system, most of neurons have a frequency receptive field (RFs) and respond 
optimally to what is called the cell's best frequency (BF). It has been frequently reported that conditioning a tonal stimulus can lead to shifts in RF such that a cell's BF, if different from the conditioned tone, shifts towards it (Weinberger, 2004, 2007). In the auditory cortex $(\mathrm{AC})$ of the guinea pig, tone-shock pairing increased sound-evoked responses to the $\mathrm{CS}$ frequency and decreased responses to other frequencies, therefore shifting BF towards the frequency of CS (Bakin and Weinberger, 1990). The absence of shift in animals with unpaired tone-shock indicates that the stimuli-specific RF shift is caused by associative learning.

The topography of neurons in the auditory system are systematically organized in a socalled "tonotopic map". The ordered distribution of BFs in tonotopic maps can be found in each auditory station. The RF plasticity of neurons is, therefore, accompanied by modification of tonotopic map, reflected by reorganization of the auditory representation (Bieszczad and Weinberger, 2010). Cortical map reorganization is depended on the specific acoustic feature that is trained. Polley et al. (2006) trained rats to do discrimination tasks with a given set of stimuli that varied in both frequency and intensity. Some rats were asked to do a frequency-based discrimination while others were required to do an intensitybased discrimination. Interestingly, attending to frequency expanded the cortical representation of the target frequency, whereas attending to intensity increased the area that tuned to the target intensity while leaving the tonotopic map intact (Polley, 2006).

Further studies that used reward, instead of electric shock, show that the level of reward is strongly correlated with the magnitude of plasticity. Rats with different level of water deprivation were trained in an auditory task in which the conditioned stimulus (CS) was coupled with water reward. The deprivation level was found to be positively correlated with performance. Mapping of the primary auditory cortex (A1) showed an expansion in CS representational area. The level of expansion also related to the level of water deprivation (Rutkowski and Weinberger, 2005). This, therefore, indicates the amount of representation change in the auditory system may code for the behavioral relevance of the acoustic stimulus.

Learning induced plasticity in the auditory cortex is usually long lasting, i.e. days or weeks (Weinberger, 2004) and can develop rapidly. Early studies in the guinea pig found that shift in RF can be obtained within the first five conditioning trials (Edeline and Weinberger, 1993). More recently, training ferrets to detect a pure tone from broadbandnoise background can elicit a rapid change in the spectro-temporal receptive field (STRF) of the neurons in the auditory cortex (Fritz et al., 2003). 
So far, long lasting plasticity is mainly observed in the cortex, while in the subcortical areas, including auditory thalamus (medial geniculate body, MGB) and the inferior colliculus, short-lasting RF plasticity has been found. In guinea pig, RF of MGB neuron can be specifically shifted towards the conditioned stimulus after classical fear conditioning (Cruikshank et al., 1992; Edeline and Weinberger, 1991). This change appeared immediately after training and lasted only about an hour. A similar pattern has been reported in the inferior colliculus. In bat, the tone-shock pairing induced CS-specific shift in RF in both auditory cortex and the inferior colliculus. The RF shift in the IC developed faster and only lasted about three hours, whereas the shift in the cortex lasted more than a day (Gao and Suga, 1998, 2000). The RF shift in IC was found to be depended on the corticofugal projection from the cortex (Gao and Suga, 1998), even though the shift in IC started earlier than the cortex (Gao and Suga, 2000). The results led the authors to hypothesize that the pairing of a sound with a shock during conditioning activates auditory cortex and elicits RF shift in the IC through the corticofugal projection, which in turn produces RF shift in the cortex through a positive feedback loop. Termination of this positive feedback loop is accompanied by reversal of plasticity in the IC. This view remained to be challenged (Weinberger, 2004), however, it points that plasticity does happen in the inferior colliculus. Recent study in the lab also shows that even long-lasting plasticity can occur in the inferior colliculus after exposure to predictable sounds (CrucesSolís et al., 2018).

It should be noted that although sensory plasticity has been extensively studied and has been found to occur in multiple sensory modalities, our understanding of how those changes link to perception and influence behavior is still limited. The plasticity in the auditory system is usually a tradeoff, such that the increase in responses or representation areas to a given stimulus is also accompanied by a decrease in responses or representation areas to flanking stimuli (Weinberger, 2004). It may, therefore, lead to imbalance in detection or discrimination abilities. Shift in RF could well be a byproduct of selective increase in neuronal responses to behavioral relevant stimulus to facilitate learning and memory processes. At the synaptic level, Long-term potentiation (LTP), which is believed to be involved in information storage, occurred more strongly when presynaptic inputs got stronger and more synchronized (Caporale and Dan, 2008). This might explain why the greater the reward, the stronger the changes in the auditory system, inducing more salient learning and better performance (Rutkowski and Weinberger, 2005). 
The environment is complex, an organism needs to constantly learn and update learnt information. Indeed, plasticity could also happen in a context-dependent manner, such that the organism can adapt quickly according to its current behavioral context. However, this can only be examined when the organism is actively performing. So far, most of those reviewed studies come from anesthetized or passive listening awake animals, in the next part we focused on how plasticity occurred in behaving animals.

\section{I.III Task-related modulation in the auditory system}

A number of works has described that neural responses, mostly in the auditory cortex, can be modulated by various behavioral and cognitive factors, such as task-engagement, context, arousal state, decision-making, and etc. (Bagur et al., 2018; Fritz et al., 2003; Osmanski and Wang, 2015; Otazu et al., 2009; Rodgers and DeWeese, 2014; Scheich et al., 2011; Schneider et al., 2018; Shimaoka et al., 2018). Many studies show increased stimulus-driven activity during behavior (Scott et al., 2007), while suppression in response has also been reported (Otazu et al., 2009).

Early work in monkeys found that sound-driven activity of single neurons was enhanced in both primary and secondary auditory cortex of animals that were actively performing an auditory detection task (Miller et al., 1972). Less or no enhancement was observed in trained but not-performing or untrained animals, respectively. The task required the monkey to keep pressing a telegraph key and only release it after a sound stimulus. A later study by the same group further investigated how activity of single neurons was modulated in different task conditions (Beaton and Miller, 1975). They trained monkeys to do similar key-release tasks, in one condition the task required detection of any tonal stimuli, while in another condition the monkey needed to selectively respond only to one tone (discrimination). $25 \%$ of the sampled cortical neurons showed alteration in the evoked response to the same tonal stimulus when switching from simple detection to discrimination task. Similar changes have also been observed in A1 of rodents (Rodgers and DeWeese, 2014). Rats were trained in two task conditions with the same set of stimuli, a combination of broadband noise played from either left or right speaker and warble of either high or low pitch played from both speakers. The task required the rat to make left-right choices based on the sound stimuli. In one condition the rat had to localize the broadband noise, whereas in another one they needed to discriminate the pitch of the warble. Some neurons selectively fired more in either of the task conditions. The selective 
neurons were found in both A1 and the prefrontal cortex, with a larger proportion in the latter, suggesting a "top-down" mechanism.

Suppression in neural activity during task engagement has also been reported. Otazu et al. (2009) trained mice to do a two-alternative choice auditory discrimination task, in which the localization of the target sound, a broadband noise, indicated the position of the reward, either left or right. A non-target stimulus, a train of clicks, appeared prior to the target stimulus. Compared to when the mouse was passive listening to the same stimuli, task engagement suppressed sound-evoked responses to both target and non-target sounds. Different from studies which found stimulus-specific modulation, this suppression was unspecific. Similar results have been reported in other studies (Atiani et al., 2009; Beitel et al., 2003; Fritz et al., 2003).

The diverse observation of task-related modulation in the auditory system may be caused by the various acoustic stimuli used in the task, task demands, training paradigm, and so on. For example, the different features of stimuli will activate distinct neuron populations and probably in different auditory stations, i.e. the tonotopic axis of amplitude modulation rate is in an orthogonal map with respect to the frequency tonotopy (Langner et al., 1997).

Apart from neural response change, rapid change in RF has also been confirmed in experiments in which the STRF was characterized during behavior (Atiani et al., 2009; David et al., 2012; Fritz et al., 2003). Water deprived ferrets were trained in an avoidance task in which they could lick continuously when a reference sound was playing but had to stop drinking when the target sound, a pure tone of a fixed frequency, appeared. The presentation of the reference sound, broadband noise-like stimuli with spectro-temporally modulated envelopes, helped to characterize the STRF during task performance. It is worth noting that the frequency of the target tone used in the task varied from day to day. Task engagement has been found to locally facilitate activity to a specific target frequency in $72 \%$ of A1 single neurons during task engaging (Fritz et al., 2003). The change in STRF happened in two ways either increasing the response to the target frequency or suppression responses to its neighbor frequencies. In some of the neurons, this change can persist for hours after the end of the task. More recent, David et al. (2012) found that the direction of change in STRF is dependent on the task demands. They trained ferrets to do either avoidance or approach task with the same set of task stimuli. The ferret needed to stop licking (avoidance) or start licking (approach) when detecting the target tone. As reported by Fritz et al. (2003), engaging in an avoidance task specifically facilitated the response to 
the target sound, whereas attending an approach task decreased that response. It indicates a connection between the change in the sensory system and the motor output (go/no-go), suggesting feedback projections from brain regions involved in decision-making.

Task-related change in neural activity has also been reported in subcortical structures, including the auditory thalamus (Jaramillo et al., 2014; Otazu et al., 2009) and the inferior colliculus (Slee and David, 2015). Jaramillo et al. (2014) observed changes in the medial geniculate body (MGB; auditory thalamus) in auditory categorization task. Rats were trained to do a two-choice frequency discrimination task, in which left was paired with low-frequency and right was paired with high-frequency. In a different block of trials, a different set of sounds was tested: 6 and $14 \mathrm{kHz}$ or 14 and $31 \mathrm{kHz}$. Thus, the same tone of $14 \mathrm{kHz}$ was paired with different behavioral choice in different blocks. In both AC and MGB, about $16 \%$ of neurons showed action-related modulation which was similar between regions. However, comparable changes occurring in both cortical and subcortical regions have not always been observed. Otazu et al. (2009) found engaging in an auditory discrimination task increased spontaneous activity in the thalamus of rats, while the evoked activity in the auditory cortex was suppressed. In a recent study in the inferior colliculus, head-fixed ferrets were trained to stop drinking when a pure tone target sound was played among reference noise. Engaging in the auditory detection task induced local and global suppression in the IC (Slee and David, 2015), whereas in the same task both suppression and facilitation have been reported in the A1 (David et al., 2012). Those results may suggest a task-related modulation in neural activity along the hierarchy of the auditory system. However, where the modulation originates and the mechanisms of the differential modulation in different stations are issues that are not yet clear.

\section{I.IV The inferior colliculus}

The inferior colliculus (IC) locates in the midbrain tectum immediately caudal to the superior colliculus. The IC can be subdivided into three main areas: a central nucleus (CNIC), a dorsal cortex (DCIC) that surrounds the CNIC dorsally and caudally and an external cortex (ECIC) that is lateral to the CNIC. The subdivision is mainly based on molecular methods but can also be identified electrophysiologically. CNIC neurons have low thresholds to simple acoustic stimuli, and the sharp tuning and short latency also distinguish them from neurons in DCIC (Winer and Schreiner, 2005).

Neurons in the CNIC show a characteristic laminar organization that has been found across species (Winer and Schreiner, 2005). The laminar structure composed of parallel 
oriented dendrites and afferent lemniscal fibers provide the substructure of the tonotopic map. Neurons in the same lamina show similar RFs. The low-frequency to high-frequency laminae are organized in the dorsal-ventral axis of the CNIC, thus creating the tonotopic map of CNIC. Tonotopy also exist in the surrounding cortex of IC. However, the border between subdivisions is not characterized by a reversal of the direction of tonotopy as is the case in cortical areas.

\section{I.IV.I The hub of auditory integration in the midbrain}

Auditory information carried by the cochlear nerve is sent to a number of different cochlear neurons, which innervate a number of parallel ascending projections to more than 10 brainstem auditory centers. Projections from those centers together with direct input from the cochlear nuclear further converge on the auditory midbrain, the inferior colliculus (reviewed in Casseday et al., 2002). Many of these inputs are inhibitory and they terminate more densely in the ventral portion of the IC (Malmierca, 2004).

The IC on both hemispheres is connected through a large number of commissural fibers. The commissural inputs terminate densely in the dorsal portion of the IC. CNIC projects to the surrounding cortex of IC of both sides but with heavier projection to the ipsilateral side. The commissural and intrinsic projections tend to follow the laminar arrangement (Casseday et al., 2002).

In addition to the feed forward sensory input, the IC receives even heavier innervations from the auditory cortex (Doucet et al., 2003; Malmierca, 2004), which arise from pyramidal cell in layer V. Similar to commissural inputs, the afferent input from AC terminates densely in the dorsal IC, and to less extend in the CNIC. Like the ascending input into the IC, corticofugal projections terminate in a tonotopic manner, that are parallel to the fibrodendric iso-frequency laminar (Casseday et al., 2002). The major corticofugal projections are glutamatergic (Ono et al., 2017; Potashner et al., 1988). However, electric stimulation experiments in cats show that AC elicits both excitatory and inhibitory effect on IC neurons projecting to MGB (Mitani et al., 1983), suggesting a direct cortical control on colliculogeniculate neurons. Further experiments in echolocating bats revealed that enhancing cortical activity led to increase of threshold, sharper tuning, and a decrease of rate-intensity functions (Suga et al., 2000), whereas suppression of cortical activity led to a reversed effect (Sun et al., 1996). Comparing electrophysiological and pharmacological methods to inhibit or facilitate IC neurons, corticofugal projection may selectively target IC inhibitory circuits (Jen et al., 1998). Activating corticofugal projection can also lead to 
frequency-specific change in the spectral tuning of collicular neurons (Suga, 2012; Suga et al., 2002; Wu and Yan, 2007; Yan and Ehret, 2001, 2002). The change in tuning depends on the similarity between the tuning of neurons in the cortex and IC. Shift in neuron best frequency (BF) in IC only occurred when its BF was different from the neuron in the cortex (Suga, 2012). Inactivation of the auditory cortex had no effect on tuning properties of IC neurons (Gao and Suga, 1998; Suga, 2012).

The inferior colliculus integrates ascending auditory inputs, descending projections from the auditory cortex as well as non-auditory projections (see below). The ascending projections from the IC terminate in its main target, the medial geniculate body (MGB), as well as lower auditory centers, including the cochlear nucleus and superior olivary complex (SOC), and some non-auditory nuclei. The CNID projects most strongly to the laminated ventral division of $\mathrm{MGB}$ in a tonotopic manner. Although most of colliculogenicular input is glutamatergic, there are also GABAergic projections. In rats, about $45 \%$ of neurons in the CNIC that projects to the MGB are GABAergic (Peruzzi et al., 1997). Those neurons have a unique morphology structure (larger in diameter) and have dense glutamatergic inputs that distinguish them from the other gamma-aminobutyric acidergic (GABAergic) neurons (Ito and Oliver, 2012; Ito et al., 2009; Ono et al., 2017). The dense excitatory inputs and their large size indicate that these neurons may show distinct neuronal response to sound and might be important in regulating sound-evoked activity in the MGB.

\section{I.IV.II Non-auditory signals in IC}

Anatomical and physiological evidence show neurons in the IC also receive input information from non-auditory centers, including the somatosensory system, ventral tegmental area (VTA), and visual cortex (reviewed in Gruters and Groh, 2012). For example, the ECIC receives bilateral projections from the VTA (Herbert et al., 1997), while CNIC and the surrounding cortex receive ipsilateral inputs from the basal nucleus of the amygdala (Marsh et al., 2002). These regions have been reported in numerous studies to be related to reinforcement learning, motivated behavior, and emotion (Davis, 1992; Fields et al., 2007; Hu, 2016; LeDoux, 2000; Salzman and Fusi, 2010). Given that auditory cortex can be modulated by amygdala (Chavez et al., 2013), these projections to IC may suggest that behavioral relevant information coding occurs in the IC. 


\section{I.IV.III Basic function of IC neurons}

Electrophysiological recording to pure tone stimuli revealed a fundamental tonotopic organization in the IC (Winer and Schreiner, 2005). Within each iso-frequency lamina, a narrow range of BFs are represented (Schreiner and Langner, 1997). Moreover, both spectral and temporal acoustic features are represented within laminae with highly organized structure (Ehret and Merzenich; Hage et al., 2003; Schreiner and Langner, 1997; Winer and Schreiner, 2005). Neuron selectivity for a certain spectral and temporal features is found to originate in the IC, like frequency modulation direction and rate (Hage et al., 2003; Kuo and Wu, 2012). Binaural processing which encodes interaural intensity and time difference starts in the SOC, and further processing in the IC (Kelly et al., 1991).

To conclude, associative learning has been shown to interact with sensation in the auditory system: both long-term and short-term plasticity occurs in the auditory system; behavioral state and relevance of stimuli can also influence neural activity. The changes in the auditory cortex have been extensively studied. However, relative little attention has been put on the subcortical areas along the information processing hierarchy. We are particularly interested in the inferior colliculus, a major processing center in the auditory midbrain. As the first convergence center of ascending and descending auditory information (Malmierca, 2004), the inferior colliculus processed dense intrinsic connections, also receives a projection from non-auditory areas (Gruters and Groh, 2012), such as the somatosensory and motor cortex. Accumulating physiological evidence show that a lot of processing already starts in the IC. The anatomical and physiological evidence suggests an integration role of IC, which may serve as a good candidate for the interface between sensation and associative learning during the encoding of behavioral relevant auditory information.

\section{I.V The general question and the particular questions we want to address}

The general question that we are interested in is how and where sensation and memory interact in the auditory system. In the current doctoral project, we aim to understand how the pre-wired auditory circuits influence learning and at which auditory station this interaction originates. With this aim we performed behavioral experiments to better understand how mice perceive auditory stimuli and experiments in which behavior and 
acute and awake electrophysiology were combined to better understand plasticity in the inferior colliculus. Behaviorally, we investigated how the physical properties of acoustic stimuli used in memory-based auditory task influenced animals' behavior, like learning and generalization (chapter 1 and 2). On the other hand, we hypothesize that the IC is the site where behavioral relevant information starts to be encoded in the auditory system. We used electrophysiological techniques to investigate whether and how neural activity in the IC was modulated by reinforcement learning and non-reinforcement learning. More specifically, we recorded neural activity of freely behaving mice trained in an auditory discrimination task to study task-related modulation in the IC (chapter 3). We also studied the effect of movement-related sound exposure on neural activity and tonal representation in the IC in both anesthetized and freely behaving mice (chapter 4).

Chapter 1 is a modified version of a submitted manuscript. The supplementary figures are embedded in the results and the numbering of figures has been reorganized. The manuscript was written by me and then edited together with my supervisor Livia de Hoz. 


\section{MATERIALS AND METHODS}

\section{II.I Animals}

Female C57BL/6JOlaHsd (Janvier, France) mice were used for the majority of experiments. In one experiment (chapter 1), Neuroligin 2 knockout (Nlgn2 KO) mice (Babaev et al., 2016; Varoqueaux et al., 2006) were used. Nlgn2 KO mice were generated on a 129/Sv background and were backcrossed onto a C57BL/6J background for at least six generations. Female wildtype (WT) and homozygous (KO) littermates were obtained from Nlgn2 heterozygous breeding pairs and provided by Dilja Krueger-Burg (Dept. Molecular Biology, MPI Experimental Medicine). All mice were 5-6 weeks old at the beginning of the experiment. Animals were housed in groups and in a temperaturecontrolled environment $\left(21 \pm 1^{\circ} \mathrm{C}\right)$ on a $12 \mathrm{~h}$ light/dark schedule $(7 \mathrm{am} / 7 \mathrm{pm})$ with access to food and water ad libitum.

\section{II.II Memory-based discrimination learning and generalization in the Audiobox}

\section{II.II.I Apparatus: the Audiobox}

The Audiobox is a device developed for auditory research and based on the Intellicage (NewBehavior, Switzerland). Mice were kept in groups of 6 to 10 animals. At least one day before experimentation, each mouse was lightly anaesthetized with Avertin i.p. $(0.1 \mathrm{ml} / 10 \mathrm{~g})$ or isoflurane and a sterile transponder (PeddyMark, $12 \mathrm{~mm} \times 2 \mathrm{~mm}$ or $8 \mathrm{~mm} \times$ $1.4 \mathrm{~mm}$ ISO microchips, $0.1 \mathrm{gr}$ in weight, UK) was implanted subcutaneously in the upper back. Histoacryl (B. Braun) was used to close the small hole left on the skin by the transponder injection. Thus, each animal was individually identifiable through the use of the implanted transponder. The Audiobox served both as living quarters for the mice and as their testing arena.

The Audiobox was placed in a soundproof room which was temperature regulated and kept in a $12 \mathrm{~h}$ dark/light schedule. The apparatus consists of three parts, a home cage, a drinking 'corner', and a long corridor connecting the other two parts (Figure 0-1A). The home cage serves as the living quarter, where the mice have access to food ad libitum. Water is available in two bottles situated in the drinking 'corner', which is positioned inside a sound-attenuated box. Presence of the mouse in the 'corner', a 'visit', is detected by an antenna located at the entrance of the corner. The antenna reads the unique 
transponder carried by each mouse as it enters the corner. A heat sensor within the corner senses the continued presence of the mouse. An antenna located at the entrance of the corner detects the transponder in the back of the mouse as it enters. The mouse identification is then used to select the correct acoustic stimulus. Once in the 'corner', specific behaviors (nose-poking and licking) can be detected through other sensors. All behavioral data is logged for each mouse individually. Access to water is controlled by opening or closing the doors behind nose-poking ports. Air puff is delivered through an automated valve which is place on the ceiling of the 'corner'. A loudspeaker (22TAF/G, Seas Prestige) is positioned right above the 'corner', for the presentation of the sound stimuli. During experimentation, cages and apparatus were cleaned once a week by the experimenter.

A
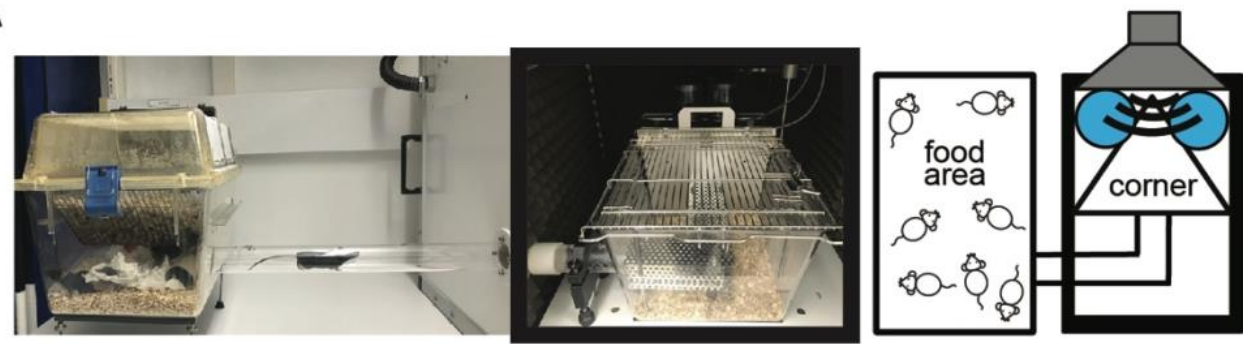

B
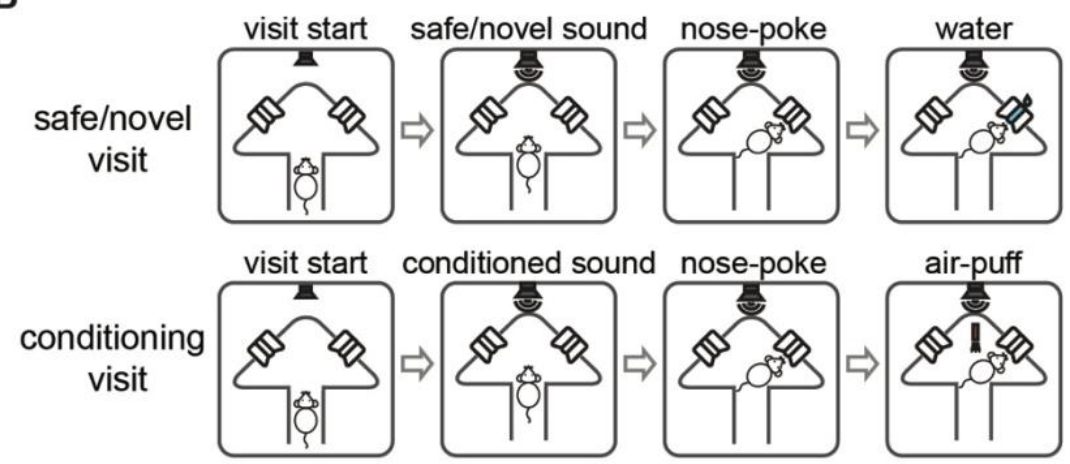

C
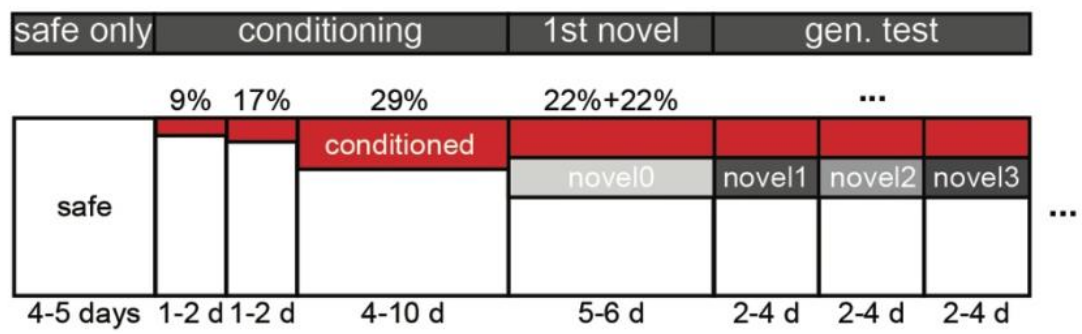

Figure 0-1 Absolute-judgement based discrimination protocol and Audiobox apparatus. (A) Photos (left) and schematic representation (Right) of the Audiobox. (B) Shema of a single safe/novel (top) and conditioning (bottom) visit. Subjects initiated a visit by entering the corner. Pure tone pips of fixed frequency presented for the duration of each visit predicted whether nose- 
poking was followed by access to water (top) or an air-puff (bottom). (C) Experimental design. The horizontal axis of the box represents time and the vertical axis represents the percentage of visits that were safe (white), conditioned (red), or novel (gray).

\section{II.II.II Sounds}

Sounds were generated using Matlab (Mathworks) at a sampling rate of $48 \mathrm{kHz}$ and written into computer files. Intensities were calibrated for frequencies between 1 and 18 kHz with a Brüel \& Kjær (4939 1/4" free field) microphone.

For experiments in chapter 1, stimuli consisted of $30 \mathrm{~ms}$ pure tone pips, with $5 \mathrm{~ms}$ rise/fall linear slopes, repeated at a rate of $3 \mathrm{~Hz}$. Tones with frequencies between 4 and 18 $\mathrm{kHz}$ were used and presented in the corner throughout the visit. Stimuli presented within a given visit had the same frequency and an intensity that roved between 67 and $73 \mathrm{~dB}$. In experiments in which we explored the effect of relative physical properties, i.e. frequency distance between the safe and the conditioned tone, on discrimination learning, we trained different group of animals (8 to 10 mice per group) with different pairs of the safe and conditioned tones. The safe tone was either 5885 or $7000 \mathrm{~Hz}$. And the conditioned tone was $0.25,0.5,075$, or 1 octave higher than the safe tone $(7000,8320,9800,11770 \mathrm{~Hz}$ for $5885 \mathrm{~Hz}$ safe tone; 8320, 9800, 11770 , or $14000 \mathrm{~Hz}$ for $7000 \mathrm{~Hz}$ safe tone). In experiments in which we investigated the effect of the relative direction of the discrimination, i.e. the difference in behavior when either the high or the low sounds are conditioned, we used either 14000 or $11770 \mathrm{~Hz}$ tone as the safe tone. The conditioned tone was in these cases 0.75 or 1 octave lower than the safe tone.

For experiments in chapter 2, mice were trained with pair of frequency-modulated (FM) sweeps or amplitude-modulation sounds or pure tone pips. Sound pairs used in training as safe and conditioned differed in two out of four chosen dimensions. For example, when the two dimensions were the frequency range of FMs and the sweep direction, the safe could be an upward sweep in the high frequency range while the conditioned would be a downward sweep in the low frequency range. For FMs, tested dimension were frequency range, sweep direction, velocity and duration. Frequency was modulated logarithmically from low to high frequencies (upward sweep) or from high to low frequencies (downward sweep). FM sweeps had a duration of $20 \mathrm{~ms}$ (default) or $40 \mathrm{~ms}$, including $5 \mathrm{~ms}$ rise/decay, and one of four modulation velocity $(50,62.5,87.5$ or 100 octave/sec; with 50 octaves/s being the default). FMs stimuli were presented at roving-intensity paradigm (68 $\mathrm{dB} \pm 3$ $\mathrm{dB})$. For AMs, tested dimensions were carrier frequency and modulation rate. AM sounds 
were $100 \%$ sinusoidal and had one of four carrier frequencies $(6670,8404,10588$ or 13340 $\mathrm{Hz}$ ), as well as one of four modulation rates $(5,8,12$ or $20 \mathrm{~Hz})$. For pure tone pips, the dimension of tone frequency and repetition rate were tested. Similar to AM, pure tones in the repetition rate experiments had one of four frequencies $(6670,8404,10588$ or 13340 $\mathrm{Hz}$ ) and one of four repetition rates $(2,3,5 \mathrm{or} 8 \mathrm{~Hz})$. Pure tones had a length of $20 \mathrm{~ms}$, with a $5 \mathrm{~ms}$ rise/fall ramp. Pure tone pips were presented with intensities that roved between 67 and $73 \mathrm{~dB}$.

\section{II.II.III Discrimination task - paradigm}

The task resembles a go/no-go discrimination task with long inter-trial intervals. Throughout the duration of the experiment, one sound (i.e. $7000 \mathrm{~Hz}$ tone) was always 'safe', meaning that access to water during these visits was granted upon nose-poke. For the first 4 days only the safe tone was played in each visit. The doors giving access to the water within the corner were open on the first day of training and closed thereafter. A nose-poke from the mouse opened the door and allowed access to water. Then the training started. Another tone of a different frequency, a 'conditioned' tone, was introduced in a small percentage of 'conditioned' visits and a nose-poke during these visits was associated with an air puff and no access to water (Figure 0-1B). The probability of conditioned visits was $9.1 \%$ for 2 days, increased to $16.7 \%$ for 2 days, then stayed at $28.6 \%$ until they showed steady discrimination performance for at least 3 consecutive days. The remaining visits continued to be safe (Figure 0-1C).

Mice that failed to learn the task, defined by no differential responses to the safe and the conditioned tone for 3 consecutive days after one week of conditioning, were excluded from the analysis. For the memory-based discrimination and generalization tests presented in chapter 1, 19 out of 177 mice were excluded. For the two-dimensional experiments presented in chapter 2,12 out of 105 mice were excluded.

\section{II.II.IV Generalization gradients for tone frequency}

Once the probability of conditioned visits reached $28.6 \%$ and animals showed stable discrimination performance (no change in performance for 3 days), we tested generalization.

During generalization testing, we introduced novel tones in a small percentage of the visits. Novel tones differed from the safe and the conditioned tone in frequency. Nose poking during the presentation of the novel tone resulted in opening of the doors that gave 
access to water. Initially, only one novel tone was presented, with a tone frequency that was at a safe-to-conditioned distance of $25 \%$ from the safe and $75 \%$ from the conditioned frequency. The first novel tone was presented for 5-8 days until mice acquired stable performance (no change in performance over 3 consecutive days) in response to the novel tone. The remaining novel sounds were introduced in pairs, two per block, with pseudorandom order and tested for 4 days each ( $\sim 50$ visits). A mouse would be thus exposed to $55.6 \%$ of safe visits, $22.2 \%$ of conditioned visits and $22.2 \%$ of novel visits $(11.1 \%$ for each of the two novel sounds) per day (Figure 0-1C). In one of the replications in which mice were trained with the $7000-14000 \mathrm{~Hz}$ pair, novel tones was presented singly for 2-3 days each with a probability of appearance of $22.2 \%$.

\section{II.II.V Multidimensional generalization measurement}

To investigate whether and how mice generalized along multi-dimension, generalization gradients were measured after stable discrimination as before.

During generalization testing, visits consisted of $55.6 \%$ of the safe visits, $22.2 \%$ of the conditioned visits and $22.2 \%$ of the novel visits in which a novel sound was presented. Since the safe and conditioned sound differed in two dimensions, novel sounds represented all possible combinations of values used (see sounds) along each dimensions. For example, when using $9 \mathrm{kHz}$ to $18 \mathrm{kHz}$ upward FM as the safe sound and $6 \mathrm{kHz}$ to $3 \mathrm{kHz}$ downward FM as the conditioned sound, tested stimuli (including the safe and conditioned sounds) resulted from factorially combining 4 different frequency ranges with 2 different sweep directions.

On each testing day, only two novel sounds were presented (in $11.1 \%$ of the visits each). Nose poking during the presentation of the novel tone resulted in opening of the doors that gave access to the water. The two diagonal sounds, each identical to either the safe or conditioned sounds in one dimensions and different in the other dimension, were always used as the first pair of novel sounds. Each novel sound was tested for 4 days with $11.1 \%$ probability ( $\sim 50$ visits).

\section{II.II.VI Analysis of performance in the Audiobox}

Data were analyzed using in-house scripts developed in Matlab (Mathwork). Performance traces for different stimuli were calculated by averaging the fraction of visits without nose-pokes over a 24-hour window. Discrimination performance was quantified by the standard measures from signal detection theory, the discriminability (d'). It was 
calculated with the assumption that the decision variables for the safe and conditioned tone have a Gaussian distribution around their corresponding mean and comparable variance. The d' value provides the standardized separation between the mean of the signal present and the signal absent distribution. It is calculated as:

$$
d^{\prime}=Z(H R)-Z(F A R)
$$

Where $Z(p), p \in\left[\begin{array}{ll}0 & 1\end{array}\right]$, is the inverse of the cumulative Gaussian distribution, HR is the hit rate, where a hit is the correct avoidance of a nose-poke in a conditioned visit, and FAR is the false alarm rate, where a false alarm is the avoidance of a nose-poke in a safe visit. Since d' cannot be calculated when either the false alarms reach levels of $100 \%$ or $0 \%$, in the few cases where this happened we used $95 \%$ and $5 \%$ respectively for these calculations. This manipulation reduced d' slightly, and therefore our d' estimates are conservative.

To evaluate the psychometric threshold and slope for pure tone generalization, we fit a sigmoid function to the normalized performance Stimuli were represented by their relative distance to the safe (-1) and conditioned (1) tones. Relative stimuli distance was calculated as:

$$
\Delta S=\frac{\left(f-f_{s}\right)+\left(f-f_{c}\right)}{f_{c}-f_{s}}
$$

Where $f$ is the frequency of the stimulus, $f_{s}$ is the frequency of the safe tone and $f_{c}$ is the frequency of the conditioned tone. In analysis for the retraining, since more than one conditioned tone was used, fitting was done to the performance in response to different frequency. Briefly, a constrained maximum likelihood method was used to fit a logistic function with 4 parameters: $\alpha$ (the $50 \%$ threshold), $1 / \beta$ (the slope of the curve), $\gamma$ (the lower asymptote), and $\lambda$ (the higher asymptote).

$$
\begin{gathered}
\psi(x)=\gamma+(1-\gamma-\lambda) \frac{1}{1+\exp (-g(x))} \\
g(x)=\frac{\mathrm{x}-\alpha}{\beta}
\end{gathered}
$$

Animals with a goodness of fit $\left(\mathrm{R}_{2}\right)$ below 0.7 were excluded from statistical analysis of threshold and slope. This was the case for 15 out of 118 animals. 


\section{II.III Frequency discrimination acuity test}

We used a modified pre-pulse inhibition (PPI) of the startle reflex protocol to measure frequency discrimination acuity as previously described (Basavaraj and Yan, 2012; Clause et al., 2011). Measurements were performed in a sound attenuated room. A schematic of the experiment setup is illustrated in Figure 0-2A. The sound was synthesized using Matlab (Mathworks), and played in a free-field 705 ultrasonic speaker (Ultrasonic Dynamic Speaker Vifa, Avisoft, Germany) through an interface (Octa capture, Roland, 704 USA) and an amplifier (Portable Ultrasonic Power Amplifier, Avisoft Germany). Simultaneously generated triggers were detected through an analog-to-digital converter data acquisition system (NI SCB-68, National Instruments, TX). The animal was positioned in a custommade chamber adjusted to the size of the mouse (length $10 \mathrm{~cm}$, inner diameter $4.2 \mathrm{~cm}$, outer diameter $5.0 \mathrm{~cm})$. The chamber rested upon a piezoelectric sensor $(30 \mathrm{~V}, 717770$, TRU COMPONENTS) for movement detection. The speaker was placed $15 \mathrm{~cm}$ away from the head of the animal. 
A

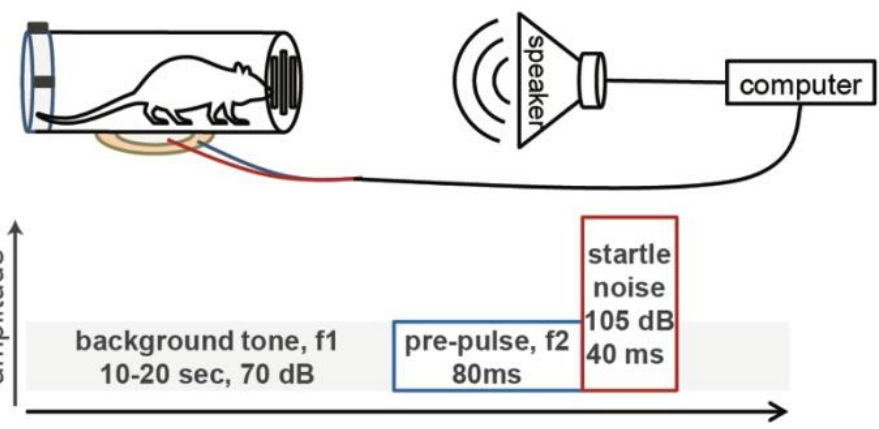

B

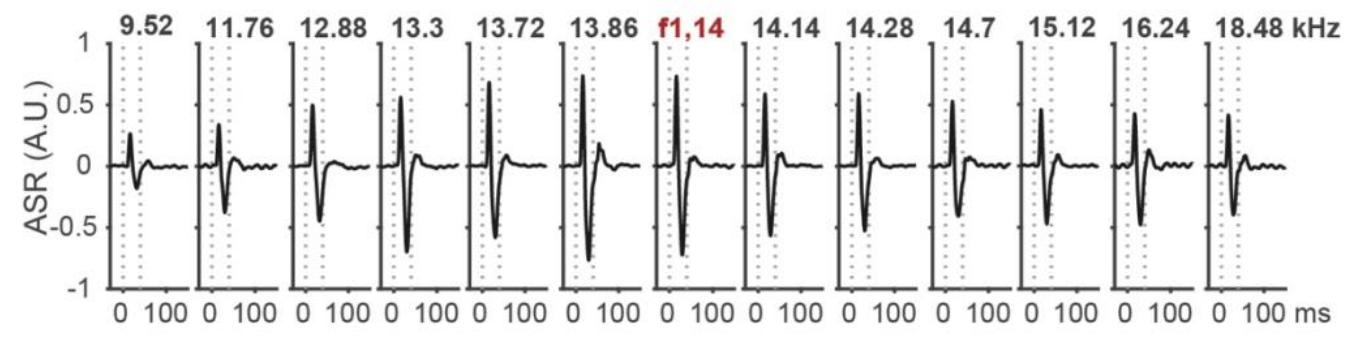

C

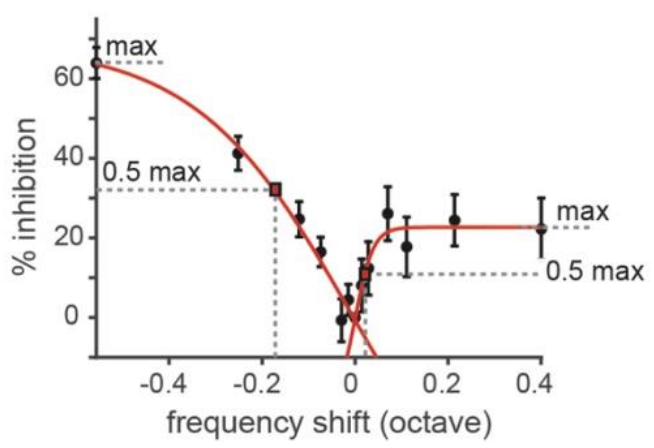

Figure 0-2 Acoustic startle apparatus for frequency discrimination acuity measurement. (A) Scheme of the acoustic startle setup (top) and a single PPI trial (bottom). PPI protocol consisted of three stimuli: background tone (f1), pre-pulse tone (f2) and the startle noise used to evoke a startle response. On each trial, a pre-pulse tone with a frequency shift of between - 0.56 and 0.4 octaves from the background tone was pseudo-randomly chosen from 13 frequencies. (B) Example average traces for one mouse represented the force measured on the platform during the PPI test for each pre-pulse tone. Background tone (labeled in red, f1) was $14000 \mathrm{~Hz}$. The magnitude of the startle response decreased as the frequency shift between the background and prepulse tone became bigger. $(\mathbf{C})$ Sample PPI curve for naïve mice $(n=10)$ tested with background tone of $14000 \mathrm{~Hz}$. Red line is the logistic fit curve (see Methods). Discrimination threshold (-0.173 and 0.022 octave for frequency below and above $\mathrm{f} 1$, respectively) was defined as a frequency shift that elicited $50 \%$ of the maximum inhibition (dash line).

The startle stimulus was a $40 \mathrm{~ms}$ broad-band noise at $105 \mathrm{~dB}$ SPL (Figure 0-2A). A background tone (f1, 70dB SPL) was presented continuously between the end of startle stimulus and the start of the pre-pulse stimulus. The pre-pulse stimulus (f2, 70dB SPL) was $80 \mathrm{~ms}$ long and consisted of a frequency change consisting of a $1 \mathrm{~ms}$ linear ramp from 
background tone, $\mathrm{f} 1$, to the pre-pulse tone, f2, also at $70 \mathrm{~dB}$ SPL. In each session, 13 frequencies were used as pre-pulse stimuli, corresponding to frequency changes $(\Delta \mathrm{f}=\log 2$ (f2/f1)) of $-0.56,-0.25,-0.12,-0.07,-0.03,-0.01,0,0.01,0.03,0.07,0.11,0.21$ and 0.40 octave, respectively.

At the start of each session, the mouse was placed in the chamber and allowed to habituate for $5 \mathrm{~min}$. This was followed by another $5 \mathrm{~min}$ of acclimation to a constant background tone (f1). The acclimation period was followed by 10 startle-only trials, 130 pre-pulse trials, and lastly by 10 startle-only trials. In startle-only trials, startle stimulus appeared directly after the background tone. In pre-pulse trials, the startle sound was immediately preceded by one of the pre-pulse stimuli. Each pre-pulse stimulus was presented 10 times in pseudo-random order. All trials had lengths varying randomly between 10 and 20 seconds.

The amplitude of acoustic startle response (ASR) was measured as the maximal vertical force exerted by the animal on the sensor during the $500 \mathrm{~ms}$ window beginning at startle stimulus onset minus the average baseline activity in the $500 \mathrm{~ms}$ window before the prepulse stimulus. The level of startle-only ASRs was calculated by averaging ASRs after f1 as pre-pulse stimuli (Figure 0-2B). To calculate PPI, the 7 strongest ASRs for each prepulse stimulus out of 10 were averaged. The level of inhibition for each pre-pulse frequency was calculated as follows:

$$
\operatorname{PPI}(\%)=100 \times \frac{\operatorname{ASRpps}_{\mathrm{f} 1}-\mathrm{ASRpps}}{\mathrm{ASRpps}_{\mathrm{f} 1}}
$$

In which $\mathrm{ASRpps}_{\mathrm{f} 1}$ is the response when pre-pulse frequency is equal to the background frequency and ASRpps is the response after pre-pulse stimulus. Discrimination threshold was defined as a frequency shift that elicited $50 \%$ of the maximum inhibition, calculated from a parametric fit to a generalized logistic function:

$$
P P I=-\frac{a}{2}+\frac{a}{1+\exp (b+c \Delta f)}
$$

The fitting was done separately for pre-pulse frequency higher or lower than the background frequency (Figure 0-2C). Curves with a fit coefficient $\left(\mathrm{R}_{2}\right)$ below 0.6 were excluded from statistical analysis. 7 lower curves and 13 upper curves out of 40 were excluded. 


\section{II.IV Electrophysiological recording with chronic implanted tetrode microdrives}

\section{II.IV.I Tetrode microdrives assemble}

The tetrode fabrication protocol was optimized based on the manual from Neuralynx. Insulated $17 \mu \mathrm{m}$ (0.00067") diameter core platinum-iridium wire (California Fine Wire) was cut to obtain four wires of $7.5 \mathrm{~cm}$ in length. The wires were twisted together using a motorized Tetrode Spinner (Neuralynx, USA). About 45 clockwise rotations were applied followed by 20 counterclockwise rotations in order to release tension on the tetrode. The final number of rotations per length of wire was about 7-8 rotations per centimeter for a 3$4 \mathrm{~cm}$ long tetrode.

In order to fuse the wires together by melting the VG bond coat, we blew air at $585^{\circ} \mathrm{C}$ using a heating gun (Neuralynx, USA) held about $5 \mathrm{~cm}$ from the wire for about $5 \mathrm{sec}$ from three different angles. To avoid melting the HML insulation and causing the wires to fuse together within the bundle we kept the heating gun moving up and down throughout. Then, we blew air at $400^{\circ} \mathrm{C}$ from about $2 \mathrm{~cm}$ distance for $10 \mathrm{sec}$ at each of three different angles.

Four tetrodes were assembled in a VersaDrive4 (Figure 0-3A; Neuralynx, USA) microdrive such that they were independently moveable. Each wire of the tetrode was gold (Gold Non-Cyanide, Sifco) plated to reduce the impedance to $200-350 \mathrm{~K} \Omega$ at $1 \mathrm{kHz}$. The tetrodes were then implanted in the inferior colliculus. 
A

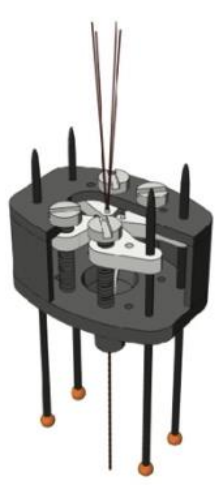

D

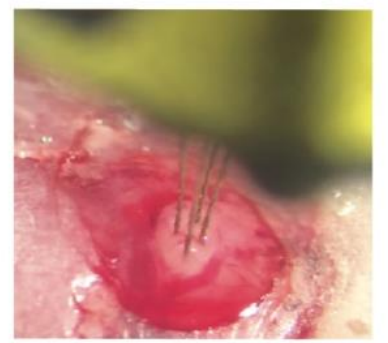

B

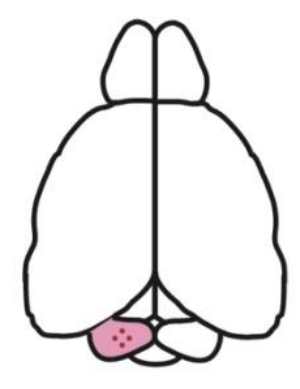

C

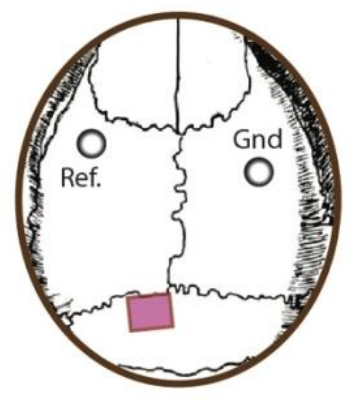

E

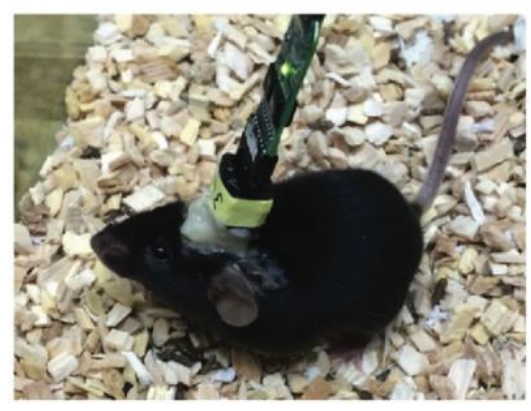

Figure 0-3 Individually moveable tetrodes implantation

(A) Schema of a semi-finished VersaDrive4, from https://neuralynx.com. (B) Four moveable tetrodes (red dots) were implanted in the left inferior colliculus (pink). (C) Shema of the craniotomy to access the left inferior colliculus. (D) Assembled VersaDrive4 with tetrode tips positioned as they would be in the central nuclei of the left IC. (E) Implanted C57BL/6j mouse plugged to the recording cable and moving freely in the recording cage.

\section{II.IV.II Implantation surgery}

To implant 4 tetrodes in the left inferior colliculus of the mouse (Figure 0-3B), animals were initially anesthetized with an intraperitoneal injection of Avertin $(0.15 \mathrm{ml} / 10 \mathrm{~g})$. Additional smaller doses of Avertin $(0.03 \mathrm{ml} / 10 \mathrm{~g})$ were added as needed to maintain anesthesia during surgery. Surgical level of anesthesia was verified by pedal-withdrawal reflex. Body temperature was maintained at $36{ }^{\circ} \mathrm{C}$ with a feedback-regulated heating pad (ATC 1000, WPI, Germany). 
After anesthesia, the animal was fixed with blunt ear bars on a stereotaxic apparatus (Kopf, Germany). Vidisic eye gel (Bausch + Lomb GmbH, Germany) was used to protect the eyes from drying out. An incision was made to expose the skull. Periosteum connective tissue that adheres to the skull was removed with a scalpel. Bone surface was then disinfected and cleaned with hydrogen peroxide. Bone suture junctions Bregma and Lamda were identified to help determine the location for tetrodes placement. Before making the craniotomy, two screwholes were drilled (OmniDrill 35, World Precision Instruments, USA) to place the ground and stabilizing screws (Figure $0-3 \mathrm{C} ; 1 \mathrm{~mm}$ in diameter). A craniotomy of $0.8 \mathrm{~mm} \times 1.0 \mathrm{~mm}$ with the center $0.85 \mathrm{~mm}$ lateral from the midline and 0.75 caudal to Lamda was made to access the left inferior colliculus (Figure 0-3C). We identified the inferior colliculus by its position which is posterior to the transverse sinus and anterior to the sigmoid sinus (Figure 0-3D). The dura was carefully removed and the brain was protected with Saline (B. Braun, Germany). We then lowered the tetrodes with a micromanipulator (Kopf, Germany), tetrodes were inserted vertically and slowly advanced (2-4 $\mu \mathrm{m} / \mathrm{sec}$, to minimize damage to the tissue) to a depth approximately $300 \mu \mathrm{m}$ from the brain surface (Figure 0-3D). The ground and the reference wires were connected to the screws, and silver paint (Silberleitlack, Ferro GmbH, Germany) was used for ensure a good connection.

After 10 to 20 minutes waiting to allow the brain to recover from the mechanical penetration, we soaked out most of the saline and covered the craniotomy and part of the tetrode cannula that extend out of the microdrive with triple antibiotic ointment (IsoptoMax, Alcon, USA). This aimed to protect the tissue from inflammation and minimize the over-growing of connective tissue, as well as avoid fixing the tetrodes by the dental resin. Finally, we applied several layers of methyl methacrylate resin (Unifast TRAD, GC). The first layer of the resin was relatively liquid to allow flowing around the insertion site. The second and subsequent layers were more viscous, leading to fast curing. After each layer of resin, we waited for 5 to 10 minutes to ensure full hardening before the next one was applied.

After the resin has completely hardened, the incision was closed with suture behind the microdrive. Antiseptic ointment (Rivanol Salbe, Dermapharm DG, Germany) was applied around the wound. $1.0 \mathrm{ml}$ warm sterile saline was bilaterally injected subcutaneously to avoid dehydration. $0.1 \mathrm{ml}$ Buprenorphine (1:30 in sterile saline) was injected as analgesia. The animal was kept in a warm place in the first few hours after surgery until full awakening. Each implanted mouse (Figure 0-3E) was housed in a separate cage to recover 
for one week. During those days, Enrofloxacin (Baytril 2.5\%, Bay Vital GmbH, Germany) water (1:125 dilution) was supplied. And mice got $0.1 \mathrm{ml}$ Buprenorphine per day in the first two days post-surgery.

\section{II.IV.III Discrimination training in the Soundterrace}

\section{II.IV.III.I Water restriction}

Mice were under water restriction protocol after complete recovery from surgery. The daily water consumption was controlled to be around 1.0 to $1.5 \mathrm{ml}$ (depending on the body weight). When animals were not trained during the weekends, ad libitum access to water was allowed (typically from Friday afternoon to Sunday afternoon). During training, mice obtained water by performing the task. If the water intake during task training was less than the designed amount of water, the rest water was given to each mouse 30 min after recording. Signs of possible dehydration were monitored (reduced skin tension, sunken eyes, and marked variation in general behavior) and were absent in all animals. To ensure adequate hydration, each animal was weighted at the beginning of daily experiment and the weight was compared to the standard weight of C57BL/6j female mice. If the weight was $<80 \%$ of the standard weight, the animal would be temporarily taken out of the study and given ad libitum water until the weight recovered. This condition never happened

\section{II.IV.III.II Apparatus: the Soundterrace}

The Soundterrace is a home-built open apparatus designed for recording in freely behaving mice, and was based on the structure of the Audiobox (TSE, Germnay). It consists of three parts: the 'arena' $(30 \mathrm{~cm} \times 30 \mathrm{~cm})$, the drinking 'corner' $(8.5 \mathrm{~cm} \times 9.5 \mathrm{~cm})$ and a corridor $(30 \mathrm{~cm} \times 5 \mathrm{~cm})$ which connects the above two parts (Figure $0-4)$. The square arena serves as the passive listening area where recordings were done when mice were not engaged in task. Water was delivered in the drinking corner though two home-built passive dispensers which use gravity as the driving force for water delivery. And that was gated by 'Normally Closed' solenoid pinch valves (225P011-11, NResearch, USA), which allow precise control of water quantities ( $\mu$ l resolution). Aversive air-puff can be delivered through a tube controlled by another solenoid valve (CH-1290 Versoix, Fluid Automation Systems, Switzerland). Two pairs of infrared sensors were mounted on two ends of the corridor (Figure 0-4, red triangles), which detected the start (close to the corner) and the end (close to the arena) of each visit to the corner, respectively. The presence of nose- 
pokes was monitored by light barriers on each nose-poke port. Experiments were controlled and monitored by custom MATLAB software together with a data acquisition board (National Instruments, NI PCIe-6321).

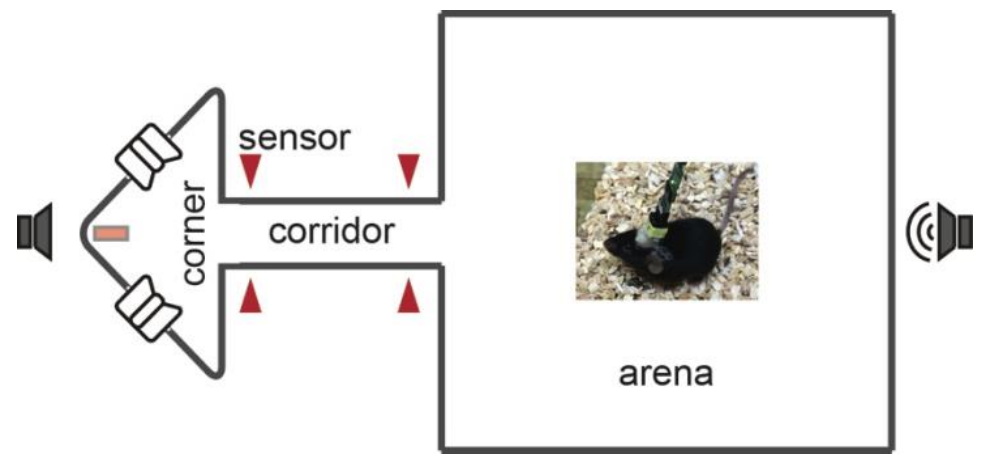

Figure 0-4 Schematic representation of the Soundterrace

The Soundterrace is home-built open apparatus designed for recording in freely behaving mice, and based on the structure of the Audiobox (TSE, Germany). It consists of three parts: the 'arena' (30 $\mathrm{cm} \times 30 \mathrm{~cm})$, the drinking 'corner' $(8.5 \mathrm{~cm} \times 9.5 \mathrm{~cm})$ and a corridor $(30 \mathrm{~cm} \times 5 \mathrm{~cm})$ which connects these two parts

\section{II.IV.III.III Sounds}

The sound was synthesized using MATLAB, produced by an USB interphase (Octa capture, Roland, USA), amplified (Portable Ultrasonic Power Amplifier, Avisoft, Germany), and played with a free-field ultrasonic speaker (Ultrasonic Dynamic Speaker Vifa, Avisoft, Germany). During behavioral training, the speaker was located $12 \mathrm{~cm}$ above the corner at 35 degree angle. During passive listening, the speaker was placed on the other side of the Soundterrace and $25 \mathrm{~cm}$ away from the center of the arena (Figure 0-4). The sound intensity was calibrated inside the corner, in the middle of the corridor as well as at the center or edge of the arena, with a Brüel \& Kjær (4939 1/4" free field) microphone. Compared with sound intensity measured inside the corner, the sound intensity in the middle of corridor was about $3 \mathrm{~dB}$ lower and the intensity at the center of the arena was about $3 \mathrm{~dB}$ lower. Within the arena, sound intensity was relatively stable, reflected by a \pm 3 $\mathrm{dB}$ differences comparing the intensity measured on the edge of the arena with intensity measured at the center.

Acoustic stimuli used for discrimination training were $30 \mathrm{~ms}$ pure tone pips with one of two frequencies, $7000 \mathrm{~Hz}$ for the safe tone and $11430 \mathrm{~Hz}$ for the conditioned tone, at $70 \mathrm{~dB}$. The onset and offset of each tone pip were smoothed with a $5 \mathrm{~ms}$ ramp. 


\section{II.IV.III.IV Discrimination training}

We trained mice to perform a pure tone frequency discrimination task in the Soundterrace. Mice were water deprived for at least ten days before the training. Similar to the Audiobox, mice trained in the Soundterrace were required to differentially respond to a safe sound that was associated with water reward and a conditioned tone that was associated with an aversive air-puff (Figure 0-5A). To increase the number of trials we achieved in one experimental day, instead of defining one visit as one trial, each visit consisted of several 13-17 seconds trials, defined by bouts of sound presentation and separated from each other by 2-5 seconds of silent breaks (Figure 0-5B). Thus, entering the corner started a visit and a series of trials, until the mouse left the corner and ended the visit. Nose-poking during silent gaps would lead to neither water reward nor air-puff punishment.

A

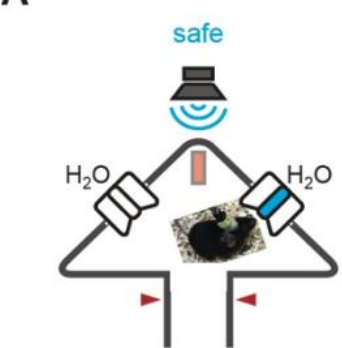

C

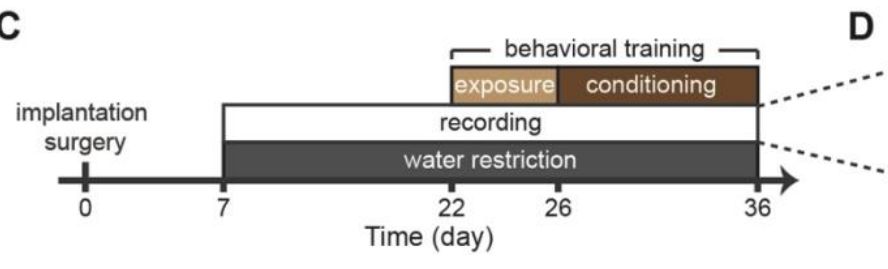

B

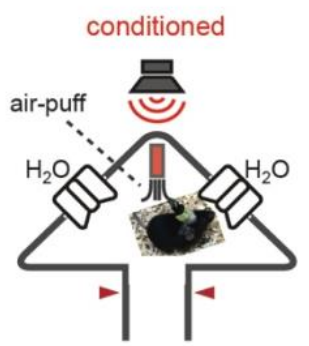

exposure-1

exposure-II

conditioning

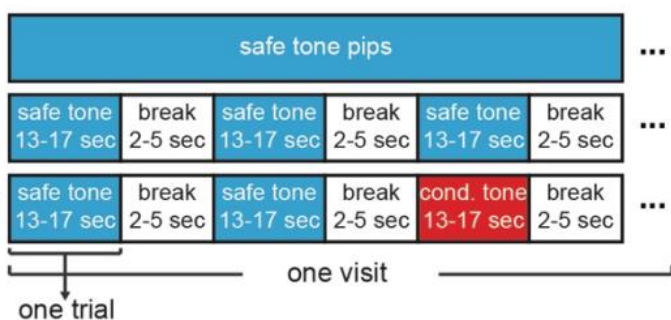

D

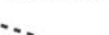

Figure 0-5 Auditory discrimination task in the Soundterrace

(A) A nose-poke during the safe tone presentation was followed by a 7-8 $\mu 1$ water reward (left), while nose-poking during the conditioned tone presentation elicited an aversive air-puff and no water reward (right). (B) Training paradigm for the exposure and the conditioning phase. (C) Experimental timeline. (D) Daily recording paradigm.

Animals were first trained on a protocol (exposure-I; Figure 0-5B)) in which only the safe sound, $7000 \mathrm{~Hz}$ tone pips, was played. This phase allowed mice to learn to access the water with a nose-poke. Detection of a nose-poke led to short opening of the valve, and 7-8 $\mu 1$ water was delivered. After two days, silent gaps were introduced to divide each visit into trials of 13 to 17 seconds. This phase (exposure-II) continued for two days, and then conditioning started. A Conditioned tone, $11430 \mathrm{~Hz}$, was played in $12.5 \%$ of the trials. Nose-poking in conditioned trials were punished with an air-puff. Once animals' 
performance reached the criteria defined as a d' value above 1, the probability of the conditioned trials was increased in steps, i.e. $12.5 \%, 14.3 \%, 16.7$, and $20 \%$.

The daily training lasted for $20 \mathrm{~min}$ for each mouse, at the same time each day. Behavioral data acquired from the Soundterrace were analyzed similarly to data analysis for the Audiobox, using in-house scripts developed in Matlab (mathwork).

\section{II.IV.IV Self-initialized sound exposure in the shuttle box}

\section{II.IV.IV.ISounds}

The sound was synthesized using MATLAB, produced by an USB interphase (Octa capture, Roland, USA), amplified (t.amp S75 mk2, Thomann, UK), and played with one of two loud speakers (22TAF/G. Seas Prestige). During sound exposure, the speakers were located on either side of the shuttle box and $15 \mathrm{~cm}$ above the box-base at 35 degree angle. During passive listening and frequency tuning measurement, an ultrasonic speaker (Ultrasonic Dynamic Speaker Vifa, Avisoft, Germany) was used and placed $15 \mathrm{~cm}$ above the base of home cage at 35 degree angle (Figure 0-6A). The sound intensity was calibrated on both sides of the shuttle box, as well as inside the home cage, with a Brüel \& Kjær (4939 1/4" free field) microphone.

A

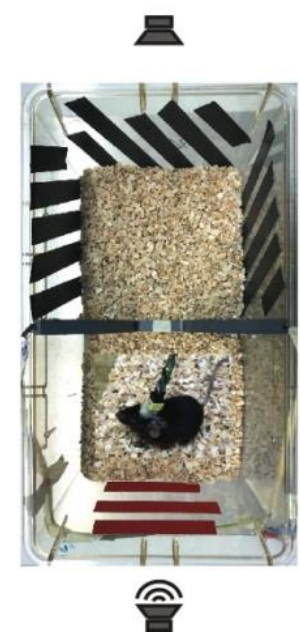

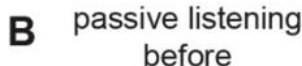
before

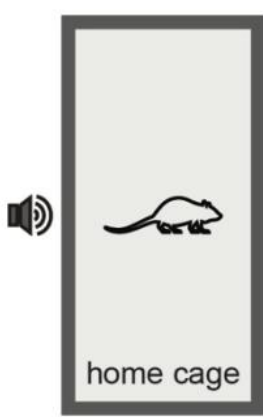

shuttle box exposure engaged 且

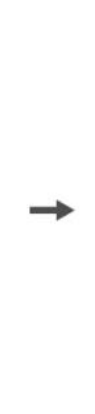

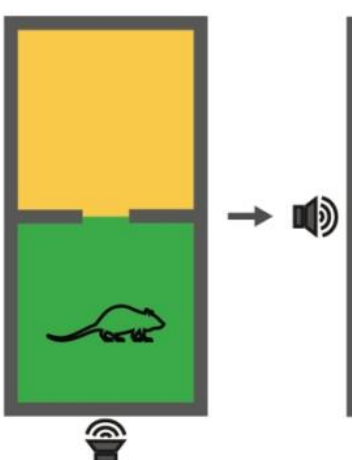

passive listening after

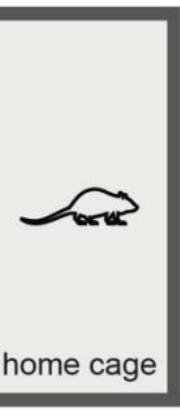

Figure 0-6 Sound exposure in the shuttle box

(A) Photo of the shuttle box. (B) Experimental design of recording in freely behaving animals during both passive listening and active sound exposure phase.

Stimuli used for sound exposure in the shuttle box were frequency-modulated (FM) sweeps. Frequency was modulated logarithmically from low to high frequencies (upward sweep) or from high to low frequencies (downward sweep). The range of frequency used 
for each mouse was chosen based on the frequency response area of the recording sites, which was $5000 \mathrm{~Hz}$ to $200000 \mathrm{~Hz}$ for mouse 1132 and $6500 \mathrm{~Hz}$ to $26000 \mathrm{~Hz}$ for mouse 1136. FM sweeps had duration of $20 \mathrm{~ms}$ or $80 \mathrm{~ms}$, including $5 \mathrm{~ms}$ rise/decay.

\section{II.IV.IV.II Sound exposure}

During sound exposure, the subjects were exposed to sounds that can be predicted by their own behavior and sounds that were not predictable. A home-built shuttle box was used for sound exposure. It was made by using a standard mouse home cage $(19 \mathrm{~cm} \times 3 \mathrm{~cm})$ as the base of the box. The box was then divided into two halves by adding a board in the middle. A $4 \mathrm{~cm}$-wide opening, the 'door', was made on the center of the board to allow animals to freely 'shuttle' from one side to the other side of the box (Figure 0-6A). Infrared sensor was mounted on the door to detect the crossing behavior. On the wall of each sub compartments, black or red stripes were taped as visual cue to distinguish those two contexts.

Each mouse was exposed to four FMs of the same frequency range (either $5-20 \mathrm{kHz}$ or 6.5-26 kHz): $20 \mathrm{~ms}$ upward FM, $80 \mathrm{~ms}$ upward FM, $20 \mathrm{~ms}$ downward FM and $80 \mathrm{~ms}$ downward FM. Each sound was played at $70 \mathrm{~dB}$ repeated at $3 \mathrm{~Hz}$ for 4 to 8 seconds. Two of the sounds were played in a predictable manner, whenever the mouse crossed the division and entered one side of the box. Thus, presentation of this 'predictable' sound was triggered by the animal's movement. The predictable sounds were always presented by a speaker close to the side being entered, the short upwards sweep when the mouse entered side A and the long downward sweep when she entered the side B (Table 0-1). The other two FM sounds (Table 0-1) were used as 'random' sounds and were presented for 4-8 seconds, 10 to 40 seconds after a crossing in a random manner independently of the movement of the animal. The random sound was played at 10 to $40 \mathrm{~s}$ intervals for as long as the animal was on a given side. Thus, the random sound could not be predicted by the mouse. In rare cases, the animal stayed in the middle of the shuttle box and did not enter either side. Under this condition, the predictable sounds for both sides were played in turns until the animal finished the crossing and stayed in one side. 


\begin{tabular}{|ccccc|}
\hline FM & upward & downward & $20 \mathrm{~ms}$ & $80 \mathrm{~ms}$ \\
\hline Predictable A & + & & + & \\
\hline Random A & & + & + & + \\
\hline Predictable B & & + & & + \\
\hline Random B & + & & & \\
\hline
\end{tabular}

Table 0-1 Acoustic stimuli used for sound exposure in the shuttle box.

Each mouse was trained in the shuttle box for 15 to 20 min every day. Experiments were controlled and monitored by custom MATLAB software together with a data acquisition board (National Instruments, NI PCIe-6321).

\section{II.IV.V Recording procedures}

One week after surgery, animals were water deprived and plugged every day to optimize the spiking activity recorded through implanted tetrodes. Each day, each tetrode was independently advanced daily about $50 \mu \mathrm{m}$ until stable spiking activity was observed.

Then animals were trained in the Soundterrace or the shuttle box to perform discrimination task or to be exposed to sounds, respectively. Immediately before and after each day's behavioral training, animals were placed in the area (Figure 0-4; for Soundterrace training, corridor was blocked) or the home cage (Figure 0-6B left; for shuttle box exposure) and passively listening to sounds. Sound stimuli consisted of $30 \mathrm{~ms}$ pure tone pips with $5 \mathrm{~ms}$ rise/fall slope repeated at a rate of $2 \mathrm{~Hz}$. For experiments in the Soundterrace, 19 frequencies were used $(6.04 \mathrm{kHz}$ to $14.6 \mathrm{kHz}, 0.071$ octave spacing) at different intensities ( $65 \mathrm{~dB}$ to $80 \mathrm{~dB}$ with steps of $5 \mathrm{~dB}$ ) played in a pseudorandom order. For experiments in the shuttle box, 24 frequencies were used $(2 \mathrm{kHz}$ to $31 \mathrm{kHz}, 0.172$ octave spacing) at different intensities ( $0 \mathrm{~dB}$ to $80 \mathrm{~dB}$ with steps of 5 or $10 \mathrm{~dB})$ played in a pseudorandom order. Each frequency-intensity combination was played 5 times. We recorded from freely behaving animals while they were performing the task, as well as during passive listening (Figure 0-5D, Figure 0-6B). After recording, animals returned to their home cage until the recording session in the next day.

The electrophysiological signal was amplified (HS-18-MM, Neuralynx, USA), sent to an acquisition board (Digital Lynx 4SX, Neuralynx, USA), and recorded with a Cheetah32 
32 Channel System (Neuralynx, USA). The raw data was acquired at $32 \mathrm{kHz}$ sampling rate with a band-pass filter $(0.1 \pm 9,000 \mathrm{~Hz})$.

\section{II.IV.VI Analysis of electrophysiological recordings}

The raw signals (Figure 0-7A) were high-pass filtered at $500 \mathrm{~Hz}$. To improve the signalto-noise ratio in the recording, the common average reference was calculated from all the functional channels and subtracted from each tetrode (Ludwig et al., 2009).
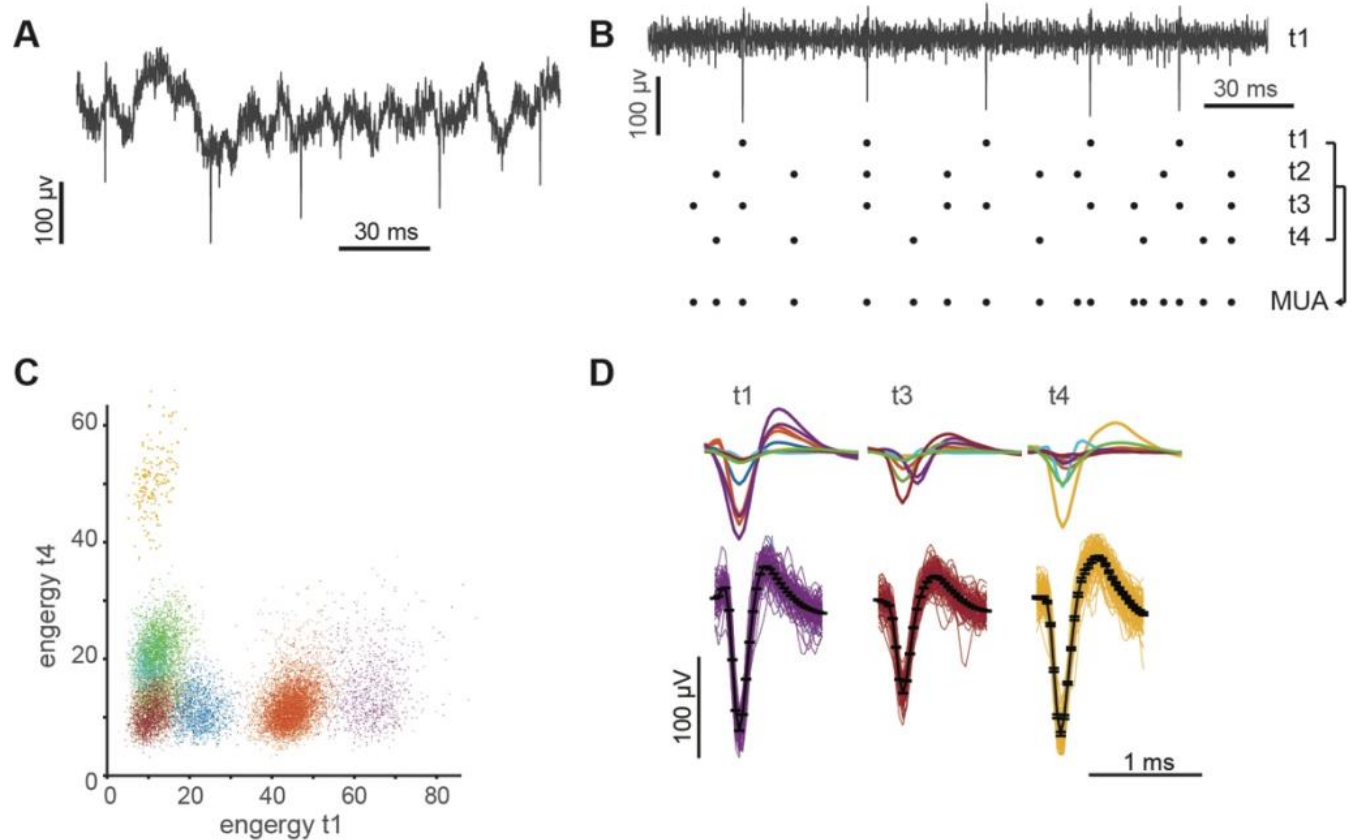

Figure 0-7 Neural activity recorded in the left IC with tetrodes

(A) Raw data trace from one wire of a tetrode. (B) Using a thresholding method to extract spikes from the high-pass filtered signal. Multiunit activity was measured by including spikes occurred in any of the four channels of a tetrode. (C) Clusters for distinct single units. (D-top) Average waveforms of distinct single units in (C) for each channel. Here, one channel of this tetrode was defected and removed from the analysis. (D-bottom) Individual raw waveforms in each channel corresponding with sorted waveforms identified in $(\mathbf{C})$. For each channel, only the cluster generated strongest negative peak was shown in the figure.

Spikes were detected as local minima below a threshold of 6.5 times the median absolute deviation of each channel (Figure 0-7B). If the calculated value was higher than $40 \mu \mathrm{V}$, the threshold was set to $-40 \mu \mathrm{V}$. When a threshold crossing event was detected in any of the four wires which belonged to the same tetrode, a $1 \mathrm{~ms}$ waveform was captured at $32 \mathrm{kHz}$ on all four channels. Automatic spike sorting was done using KlustaKwik (http://klustakwik.sourceforge.net/), with Energy (L-2 norm of the acquired waveform), Peak 6 to11 (minimum of the value in samples 6 to samples 11), Valley (maximum of the 
voltage deflection) and Peak (minimum of the voltage deflection) as clustering features. Sorted clusters were subsequently checked and adjusted manually (Figure 0-7C, D) using MClust (http://redishlab.neuroscience.umn.edu/MClust/).

For multiunit analysis, spikes were detected using the same thresholding method used for single unit analysis. Spikes were included if they occurred in any of the four channels of the tetrode (Figure 0-7B, bottom). Multiunit sites were included in the analysis (except the analysis for spontaneous activity) only if they were classified as sound driven, defined by significant excitatory evoked responses (comparison of overall firing rate in $80 \mathrm{~ms}$ windows before and after stimulus onset for all frequencies and intensities). For LFPs, we used the raw signal from one of the wires in each tetrode (Figure 0-7A).

To analyze the sound-driven responses, signals were aligned at $200 \mathrm{~ms}$ before each stimulus onset. For spikes, spontaneous activity and evoked firing rates were calculated by averaging the signal in an $80 \mathrm{~ms}$ window before and after each stimulus onset, respectively. The temporal pattern of the responses was compared using the peri-stimulus time histograms (PSTHs). The PSTHs in the figure were smoothed by a 10ms hamming window. For LFP, evoked firing rates were calculated by calculating the negative peak in an $80 \mathrm{~ms}$ window after each stimulus onset. The best frequency (BF) was selected as the frequency which elicited the highest responses when averaging across all intensities. In the rare cases where more than one frequency elicited the highest response, BF was calculated as the mean of those frequencies.

\section{II.IV.VII Histology}

After finishing the behavioral and electrophysiological experiments, the position of the electrodes was marked by generating a small lesion at the end of each tetrode. This was achieved by passing $12 \mu \mathrm{A}$ direct current for $8-10 \mathrm{sec}$ through one of the channels in each tetrode (Moshitch et al., 2006; Townsend et al., 2002). The current was derived from NanoZ (Neuralynx, USA). The magnitude of the current was set in the NanoZ software. After lesion, decapitation was used to sacrifice the mouse. The brain was gently removed from the skull and fixed in $4 \%$ paraformaldehyde. After 24 hours of fixation, the brain was cryoprotected in $30 \%$ sucrose solution and sectioned in the coronal plane at a thickness of 80-100 $\mu \mathrm{m}$ on a Vibratome. Brain slices were stained with DAPI to facilitate identification of cytoarchitectural boundaries. Digital photographic images were taken by ApoTome (Carl Zeiss Microscopy GmbH, Germany), after staining (Figure 0-8). 


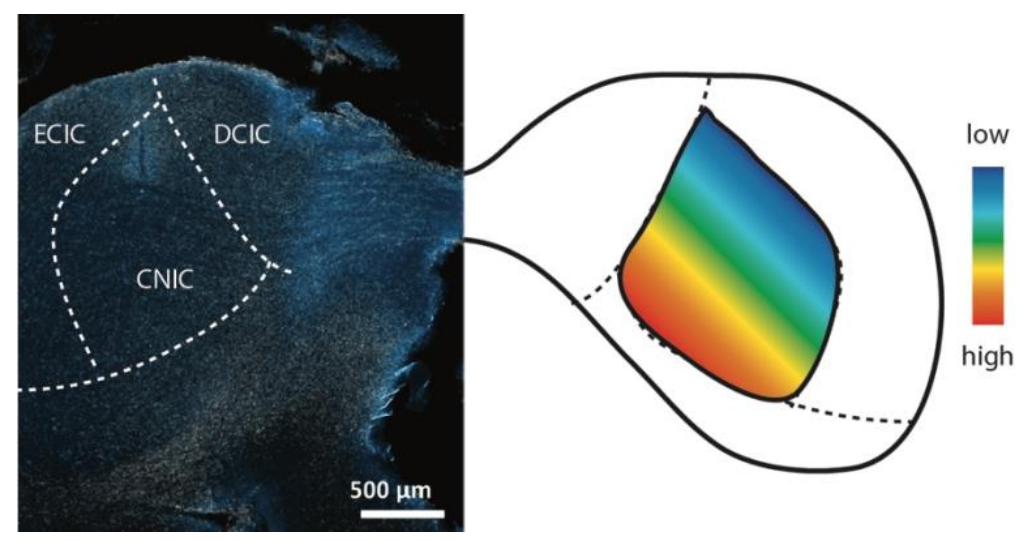

Figure 0-8 Post-recording histology

(Left) Representative dorsoventral tetrode penetration track. (Right) Schematic representation of the tonotopic map of the inferior colliculus.

\section{II.V Acute electrophysiology}

\section{II.V.I Sound exposure in the Audiobox}

Five experimental groups were used in chapter 4 for acute electrophysiological recording. All mice had ab libitum food in the home cage, as well as free access to water through nose-poking in the corner, except the 'no water exposure' group (see below) which had water in the home cage instead. There was no conditioning (air-puffs) in the Audiobox at any stage. All groups were first habituated to the Audiobox for 3 days without sound presentation. After the habituation phase, four of the groups were exposed to $16 \mathrm{kHz}$ pure tone pips in the corner with different exposure protocol.

The 'normal exposure' group had similar sound exposure paradigm as in a previous study (Cruces-Solís et al., 2018). The mice were exposed to $16 \mathrm{kHz}$ tone pips for the duration of each visit, regardless of nose-poke activity and water intake.

The 'no-water exposure' group was treated the same as the normal exposure group, i.e. it was exposed to $16 \mathrm{kHz}$ tone pips for the duration of each visit intervals. However, there was no water in the corner, and water was, unlike in all other groups, accessible in the home cage.

For the 'unpredictable exposure' group, $16 \mathrm{kHz}$ tone pips were presented in $80 \%$ of the visits and only after a random 5 to 25 seconds delay from the time of entering the corner. Once the sound started, it continued until the mouse left the corner. Thus, the starting of the sound could not be predicted by the animal

For the 'entry-only' group, $16 \mathrm{kHz}$ tone pips were triggered by the entry into the corner, but only for $3 \mathrm{sec}$. If the visit length was shorter than $3 \mathrm{sec}$, the sound stopped once the mouse left the corner. 
The control group lived in the Audiobox for the same amount of time as the other groups, but without sound presentation. All animals can hear environmental sounds, i.e. their own vocalization and the sound of the ventilation fan.

\section{II.V.II Surgery and electrodes insertion}

The surgical part of the acute recording was similar to the one for chronic implantation. Animals were initially anesthetized with Avertin $(0.15 \mathrm{ml} / 10 \mathrm{~g})$. Additional smaller doses of Avertin $(0.03 \mathrm{ml} / 10 \mathrm{~g})$ were added as needed to maintain anesthesia during surgery. Surgical level of anesthesia was verified by pedal-withdrawal reflex. Body temperature was maintained at $36{ }^{\circ} \mathrm{C}$ with a feedback-regulated heating pad (ATC 1000, WPI, Germany).

After anesthesia, the animal was fixed with blunt ear bars on a stereotaxic apparatus (Kopf, Germany). Vidisic eye gel (Bausch + Lomb GmbH, Germany) was used to protect the eyes from drying out. The scalp was removed to expose the skull. Periosteum connective tissue that adheres to the skull was removed with a scalpel. Bone surface was then disinfected and cleaned with hydrogen peroxide. Bone suture junctions Bregma and Lamda were aligned. A metal head-holder was glued to the skull $1.0 \mathrm{~mm}$ rostral to Lamda with methyl methacrylate resin (Unifast TRAD, GC). A craniotomy of $0.8 \mathrm{~mm} \times 1.0 \mathrm{~mm}$ with the center $0.85 \mathrm{~mm}$ to the midline and 0.75 caudal to Lamda was made to expose the left inferior colliculus. After the craniotomy, the brain was protected with Saline (B. Braun, Germany). The area which is posterior to the transverse sinus and anterior to the sigmoid sinus was identified as the inferior colliculus.

The borders of the left inferior colliculus became visible after the craniotomy. The electrode, a single shank 16-channel silicon probe $(177 \mu \mathrm{m} 2$ area/site and $50 \mu \mathrm{m}$ spacing between sites; Neuronexus, USA), was placed in the center of the inferior colliculus after measuring its visible rostrocaudal and mediolateral borders. The aim was to target the same position of the inferior colliculus across animals. Before lowering the electrode, a ground wire was connected to the neck muscle as a reference for the recordings. With a micromanipulator (Kopf, Germany), the electrode was vertically lowered and slowly advanced (2-4 $\mu \mathrm{m} / \mathrm{sec}$, to minimize damage to the tissue) to a depth of $750 \mu \mathrm{m}$ from the brain surface. The final depth was double checked by making sure that the most dorsal channel was aligned with the dura (Figure 0-9). After one recording session, the electrode was further advanced $500 \mu \mathrm{m}$ to a depth of $1250 \mu \mathrm{m}$. 


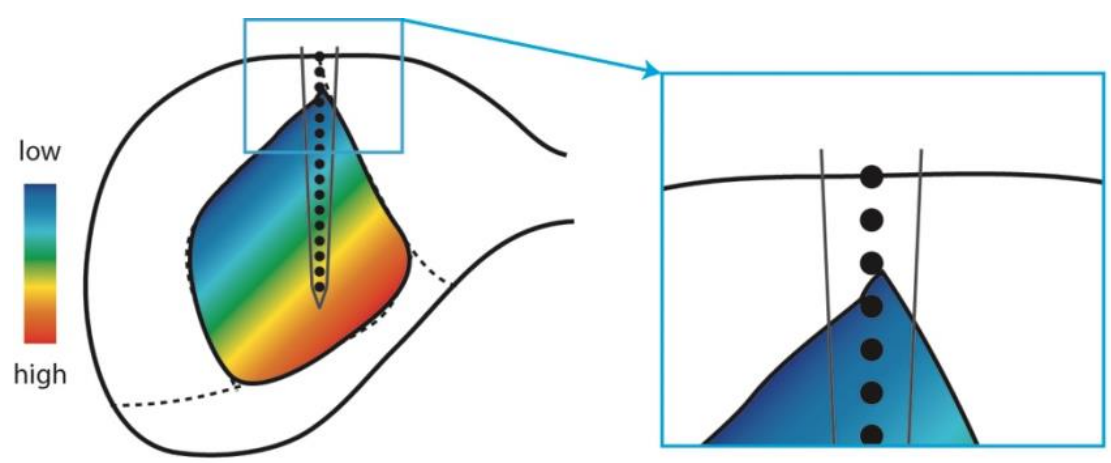

Figure 0-9 Schematic representation of recording with silicon probe in the left IC (Left) Schematic representation of the single-shank silicon probe placing during recording. (Right) Schema of positioning the most superficial recording site which was aligned with the dura.

\section{II.V.III Data acquisition}

As for the awake recording that was described above, the electrophysiological signal was amplified (HS-18-MM, Neuralynx, USA), sent to an acquisition board (Digital Lynx 4SX, Neuralynx, USA), and recorded with a Cheetah32 32 Channel System (Neuralynx, USA). The voltage traces were acquired at a $32 \mathrm{kHz}$ sampling rate with a wide band-pass filter $(0.1 \pm 9,000 \mathrm{~Hz})$.

\section{II.V.IV Acoustic stimulation during recording}

The sound was synthesized using MATLAB, produced by an USB interphase (Octa capture, Roland, USA), amplified (Portable Ultrasonic Power Amplifier, Avisoft, Germany), and played with a free-field ultrasonic speaker (Ultrasonic Dynamic Speaker Vifa, Avisoft, Germany). The speaker was $15 \mathrm{~cm}$ away from the right ear. The sound intensity was calibrated with a Bruël \& Kjaer microphone. For measuring the frequency response area, we used sound stimuli which consisted of $30 \mathrm{~ms}$ pure tone pips with $5 \mathrm{~ms}$ rise/fall slope repeated at a rate of $2 \mathrm{~Hz}$. Thirty-two frequencies were used $(3.4 \mathrm{kHz}$ to 49.4 $\mathrm{kHz}, 0.125$ octave spacing) at different intensities ( $0 \mathrm{~dB}$ to $80 \mathrm{~dB}$ with steps of 5 or $10 \mathrm{~dB}$ ) played in a pseudorandom order. Each frequency-intensity combination was played 5 times.

\section{II.V.V Analysis of electrophysiological Recordings}

The recorded voltage signals were high-pass filtered at $500 \mathrm{~Hz}$. To improve the signalto-noise ratio of the recording, the common average reference was calculated from all the functional channels and subtracted from each channel (Ludwig et al., 2009). 
For multiunit analysis, spikes were detected as local minima below a threshold of 6 times the median absolute deviation of each channel. If the calculated value was higher than $-40 \mu \mathrm{V}$, the threshold was set to $-40 \mu \mathrm{V}$. Multiunit sites were included in the analysis only if they were classified as sound-driven, defined by significant excitatory evoked responses (comparison of overall firing rate in $80 \mathrm{~ms}$ windows before and after stimulus onset for all frequencies and intensities). The raw signal from each channel was used as local field potential (LFP).

To analyze the sound-driven responses, signals were aligned at $200 \mathrm{~ms}$ before each stimulus onset. Spontaneous activity and evoked firing rates were calculated by averaging the signal in an $80 \mathrm{~ms}$ window before and after each stimulus onset, respectively. Signalto-noise ratio (SNR) was calculated as the ratio between evoked responses and spontaneous activity. The temporal pattern of responses was compared using peri-stimulus time histograms (PSTHs). PSTHs in the figure were smoothed by a $10 \mathrm{~ms}$ hamming window.

Sound-driven responses at a given intensity as a function of frequency were used to generate the iso-intensity tuning curves. The combined frequency-intensity responses were used to generate tonal receptive field (TRF).

The best frequency (BF) was selected as the frequency which elicited the highest responses when averaging across all intensities. In the rare cases where more than one frequency elicited the highest response, BF was calculated as the mean of those frequencies.

Bandwidth was calculated from $3 \times 3$ median filtered TRF ( $3 \times 3$ median filtered). The base bandwidth was the width of frequencies that evoked more than $20 \%$ of the max responses at a given intensity. The half-maximum bandwidth was the width of frequencies that evoked more than half of the max responses at a given intensity.

Reliability was calculated for each frequency-intensity combination as the percentage of trials that showed more than 1 spike in the $80 \mathrm{~ms}$ window after stimulus onset.

Intensity threshold was defined as the lowest sound intensity that elicited significant responses. Characteristic frequency was the frequency that elicited strongest response at threshold.

Neural activities at the depth from $500 \mu \mathrm{m}$ to $750 \mu \mathrm{m}$ were measured in two recording sessions, first when the probe tip was at $750 \mu \mathrm{m}$ depth and then, when it was at $1250 \mu \mathrm{m}$. When analyzing responses at given depth, recordings for the same depth were averaged. 


\section{II.VI Statistical analysis}

Group comparisons were made using multiple way ANOVAs after testing for normality distribution using the Shapiro-Wilk test. Samples that failed the normality test were compared using Wilcoxon signed rank test. Multiple comparisons were adjusted by Bonferroni correction. For analysis of data consisting of two groups we used either paired t-tests for within-subject repeated measure- ments or unpaired t-tests otherwise. For data consisting of more than two groups or multiple parameters we used, repeated-measures ANOVA. All multiple comparisons used critical val- ues from a t distribution, adjusted by Bonferroni correction with an alpha level set to 0.05. Means are expressed \pm SEM. Statistical significance was considered if $p<0.05$. 


\section{Chapter 1 Wide sensory filters underlie performance in memory-based discrimination and generalization}

\subsection{Introduction}

An animal's response to external stimuli depends largely on the animal's capacity to identify the current stimulus as the same or similar to previously encountered stimuli. Often, experimental design is such that stimuli are presented in relatively quick succession (e.g. (Delhommeau et al., 2002; Grimault et al., 2003; Kurt and Ehret, 2010; McShefferty et al., 2015)). In these conditions the memory of the physical characteristics of the stimulus presented in the previous trial might still be active and a relative-judgement can be made. For example, the study of just-noticeable-differences (JNDs) or difference limens, i.e. the minimum physical differences that still allow discrimination, has received a lot of attention across modalities and is based on experiments that rely on relative-judgement (Ehret, 1975; Fay, 1974; Johnson et al., 1993, 2002; Laming, 2010; Regan and Beverley, 1985; Skottun et al., 1987; Stone and Bosley, 1965; Talwar and Gerstein, 1998; Westheimer et al., 1976; Zanker, 1995). The general finding is that across species, animals can make fine discriminations. In the auditory domain, for example, humans and rodents can differentiate between frequencies that differ in small $\Delta$ Fs of a few percent (Ehret, 1975; Fay, 1974; Talwar and Gerstein, 1998). Similarly, in the visual domain, orientations differing in a fraction of a degree can be discriminated (Regan and Beverley, 1985; Skottun et al., 1987; Westheimer et al., 1976). From a different perspective, the study of absolute judgement (Miller, 1994), which tests the capacity of a subject to order a given stimulus among a group of stimuli varying along a single dimension, also relies on experimental designs in which stimuli are presented successively. In nature, however, animals often have to decide how to respond to a stimulus that is presented in spatial and temporal isolation from others that resemble it. This type of memory-based judgement is reflected in some forms of stimulus categorization, which has been the subject of substantial research in several species, including humans, monkeys and pigeons (Ashby and Rosedahl, 2017; Smith et al., 2016) but not in simpler discriminations. Understanding how perception of current stimuli is affected by the memory of other stimuli can help us understand the sensory filters that are in place during memory-based judgements, and infer the interaction between the 
involved neuronal populations. In the auditory domain, little is known about the role played by differences in a given physical dimension, such as sound frequency, in memorybased discriminations. In humans, tone frequency judgements, in isolation, are generally difficult unless the subject has absolute pitch but can be ameliorated with the use of reference frequencies (Smith and Schmuckler, 2008).

To further our understanding of the role played by the stimulus physical properties on memory-based discrimination in mice, we used sounds that varied along a single dimension, frequency, in a memory-based task in the Audiobox (TSE, Germany), an automatic apparatus in which mice live in groups and perform ad libitum for the duration of the experiment. We used two measures of performance to infer perception: discrimination learning and generalization of this learning to new and similar stimuli. While our main focus was on the role of a sound frequency distance in sound perception, we also measured the role of sound valence and previous training as well as the effect that training had on spontaneous frequency discrimination using the startle reflex.

\subsection{Results}

C57BL/6JOlaHsd mice were trained to perform auditory tasks in a behavioral apparatus, the Audiobox (TSE; Figure 0-1A), in which mice live for the duration of the experiment (several weeks) while performing the task ad libitum. Initially, we trained mice in a puretone frequency discrimination task that resembled a go/no-go discrimination paradigm. Mice could obtain water only in the 'corner' of the Audiobox (Figure 0-1A). On each visit to the corner, and for the duration of the visit, mice were presented with a sound stimulus consisting of a train of pure tone pips of the same frequency. In those visits in which the 'safe sound' was presented (safe visits), mice had access to water through a nose-poke on either side of corner (Figure 0-1B-top), whereas when the 'conditioning sound' was played (conditioned visits), nose-poking was followed by an aversive air puff and no access to water (Figure 0-1B-bottom). Importantly, most visits, over two thirds, are spaced by intervals of more than 1 minute (Cruces-Solis et al., 2018) and frequency judgement must, therefore, rely on memory. 


\subsubsection{Mice associated acoustic stimuli with different behavioral outcomes and generalized the learnt association to novel stimuli.}

The frequency discrimination paradigm used in our experiments is outlined in Figure 0 -1B-C. In the first experiment, mice $(n=9)$ were trained with two frequencies 1 octave apart: $7000 \mathrm{~Hz}$ as the safe sound and $14000 \mathrm{~Hz}$ as the conditioned sound. Conditioning visits were introduced after a phase of only safe visits. We calculated the average percentage of visits without nose-pokes across mice in blocks of 24 hours separately for safe and conditioned visits as a main performance index (Figure 1-1A). On average, mice did not nose-poke in about $30 \%$ of the safe visits, which is the typical baseline performance of the Audiobox (de Hoz and Nelken, 2014). Once conditioning began, mice learnt the task fast and successfully as revealed by a clear nose-poke avoidance during conditioned visits and a significant difference in the nose-poking behavior during safe and conditioned visits (6 days; a 3-way ANOVA on tone frequency, animal and day, revealed a main effect of frequency $F(1,93)=1110.41, p=0$; effect of animal $F(8,93)=2.78, p=$ 0.0084; effect of day $F(5,93)=5.1, p=0.0004)$. Already in the first day of conditioning, avoidance responses to the conditioned tone reached $79 \%$. This increased to $94 \%$ on the following day, while the avoidance responses to the safe tone remained at approximately at $35 \%$ throughout. The training continued until the probability of conditioned visits reached $29 \%$ and mice achieved a stable discrimination performance, defined by a lack of significant change in d' value for at least three consecutive days $(p>0.05)$. 


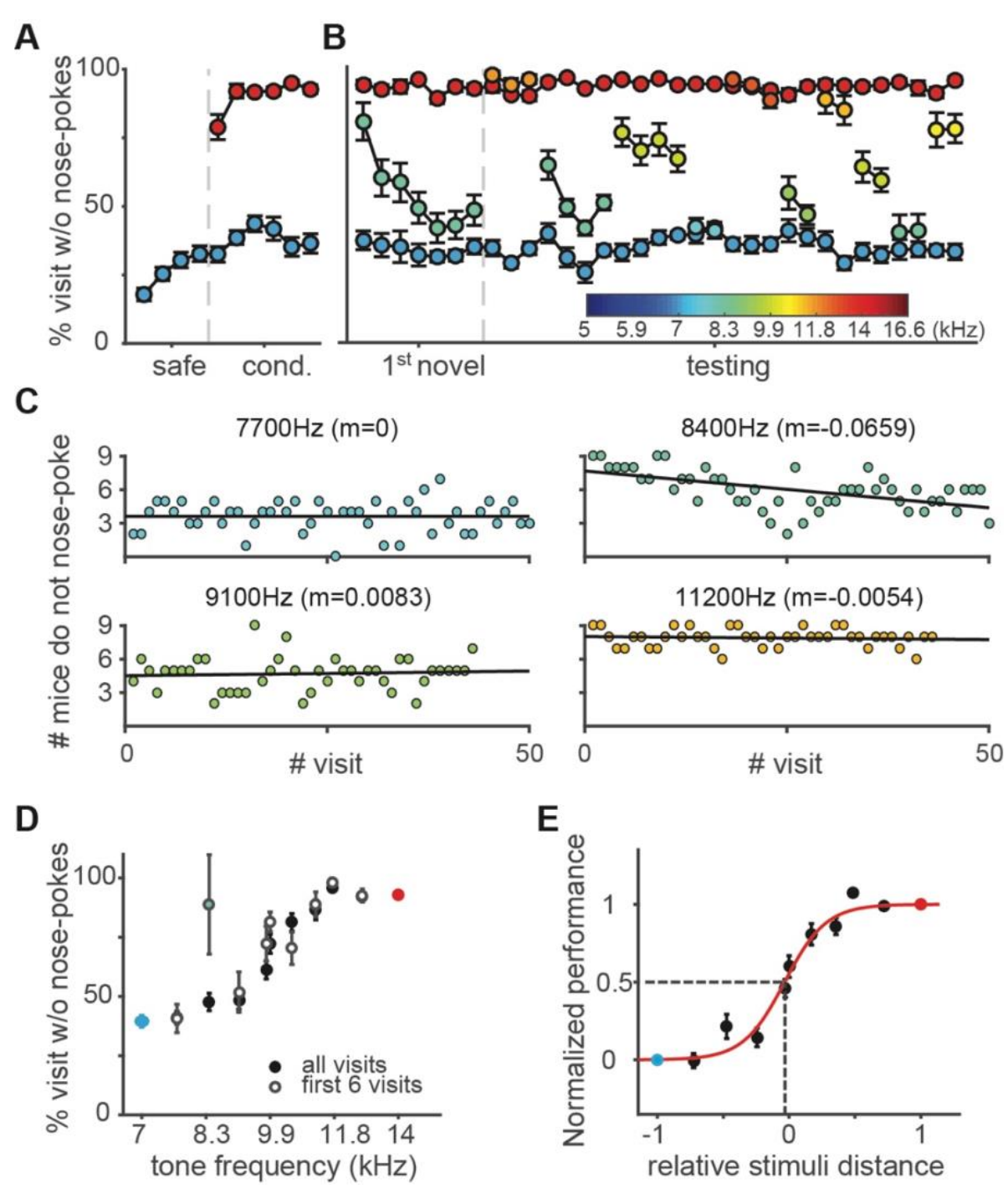

Figure 1-1 Discrimination training and generalization testing in the Audiobox

(A) Performance during discrimination training. Mean daily performance expressed as the fraction of visits without nose-pokes for the safe (blue) and conditioned visits (red). (B) Performance during generalization test. Mean daily performance for the safe (blue), conditioned (red), novel visits (color-coded by novel tone frequency). (C) Single trial performance analysis as \# mice $(n=9)$ that avoided nose-poking in each novel visit for novel visits with tones of $7700 \mathrm{~Hz}$ (top-left), $8400 \mathrm{~Hz}$ (top-right, 1 st novel tone presented), $9100 \mathrm{~Hz}$ (bottom-left) or $11200 \mathrm{~Hz}$ (bottom-right). Linear regression shown as black line, and slope, on the title. (D) Generalization gradient, average performance as a function of tone frequency, for all visits (black) and the initial 6 visits of each visit type (white). Here and in subsequent figures, red and blue closed dots indicate responses to the conditioned and safe tone, respectively. (E) Normalized performance (black dots) and fitted psychometric curve (red). Evaluated psychometric threshold as the stimulus strength for which performance is at the midpoint (dash line).

To measure generalization, we introduced 'novel' visits in $22 \%$ of the total visits. During these visits a novel tone was presented (Figure 0-1B-top). Safe visits and conditioned visits constituted now 56\% and $22 \%$ of the total visits (Figure 0-1C). Novel 
visits were actually safe, and nose-poking on either side resulted in opening of the nosepoke door and access to water. These novel tones were typically of a frequency somewhere in-between the safe and conditioned sounds and were introduced in a pseudo-random order (see Methods for detail). We started with a novel tone of $8400 \mathrm{~Hz}$ in frequency, equivalent to 0.26 octave above the safe sound. Because the conditioned sound is different in both frequency and probability of appearance, mice often treat other rare sounds as conditioned (de Hoz and Nelken, 2014). For this reason, the first novel sound was relatively close in frequency to the safe sound and was presented during at least 6 consecutive days until the animals reached a stable response in its presence. Indeed, mice avoided nose-poking during novel visits on the first day (Figure 1-1B, 1st novel). But this avoidance gradually decreased and stabilized around 50\%. Figure 1-1C shows the number of mice that avoided nose-poking in each individual novel visits for different frequencies. It is clear that while most mice avoided nose-pokes on the first presentations of the first novel sound, avoidance decreased over successive visits (Figure 1-1C, $8400 \mathrm{~Hz}$ ). For the remaining novel sounds, the level of avoidance tended to be either low or high (Figure 1-1B, testing). This was already visible from the first visit on which a novel sound was presented, and it remained at that level throughout (Figure 1-1C; $7700 \mathrm{~Hz}, 9100 \mathrm{~Hz}$ and $11200 \mathrm{~Hz}$ ), suggesting that the decision whether to approach or avoid the water port was made based on the frequency of the sound. The introduction of novel sounds did not affect the level of performance during either safe or conditioned visits (Figure 1-1B, blue and red points respectively).

To better visualize the data, we constructed psychometric curves for individual mice by plotting average percentage of visits without nose-pokes against the tone frequency. The psychometric curve took the shape of a sigmoid-function. Individual animals showed similar level of generalization around trained frequencies and more variable performance in-between them (Figure 1-2A). Mice showed generally a high level of avoidance responses to novel tones with high frequency and low level avoidance to tones with low frequency (Figure 1-1D), and this effect was present already during the first 6 visits of each sound-frequency (red dots). A 2-ways ANOVA on tone frequency and animal, revealed a main effect of frequency $(F(10,80)=66.73, p<0.0001)$ and animal $(F(8,80)=6.32, p<$ 0.0001). Therefore, learning generalized from the trained sounds to the other tones in a frequency-specific manner, independently of the fact that water was available during all novel visits and no air-puff was delivered. For frequencies below $7700 \mathrm{~Hz}$ or above 11200 $\mathrm{Hz}$, mice responded as if those visits were equivalent to safe or conditioned visits, 
respectively (Figure 1-1D; paired t-test, p7000,7700 > 0.05, p14000,11200 > 0.05, p14000,12700> 0.05 , the remaining comparisons had a p-value lower than 0.05 ).
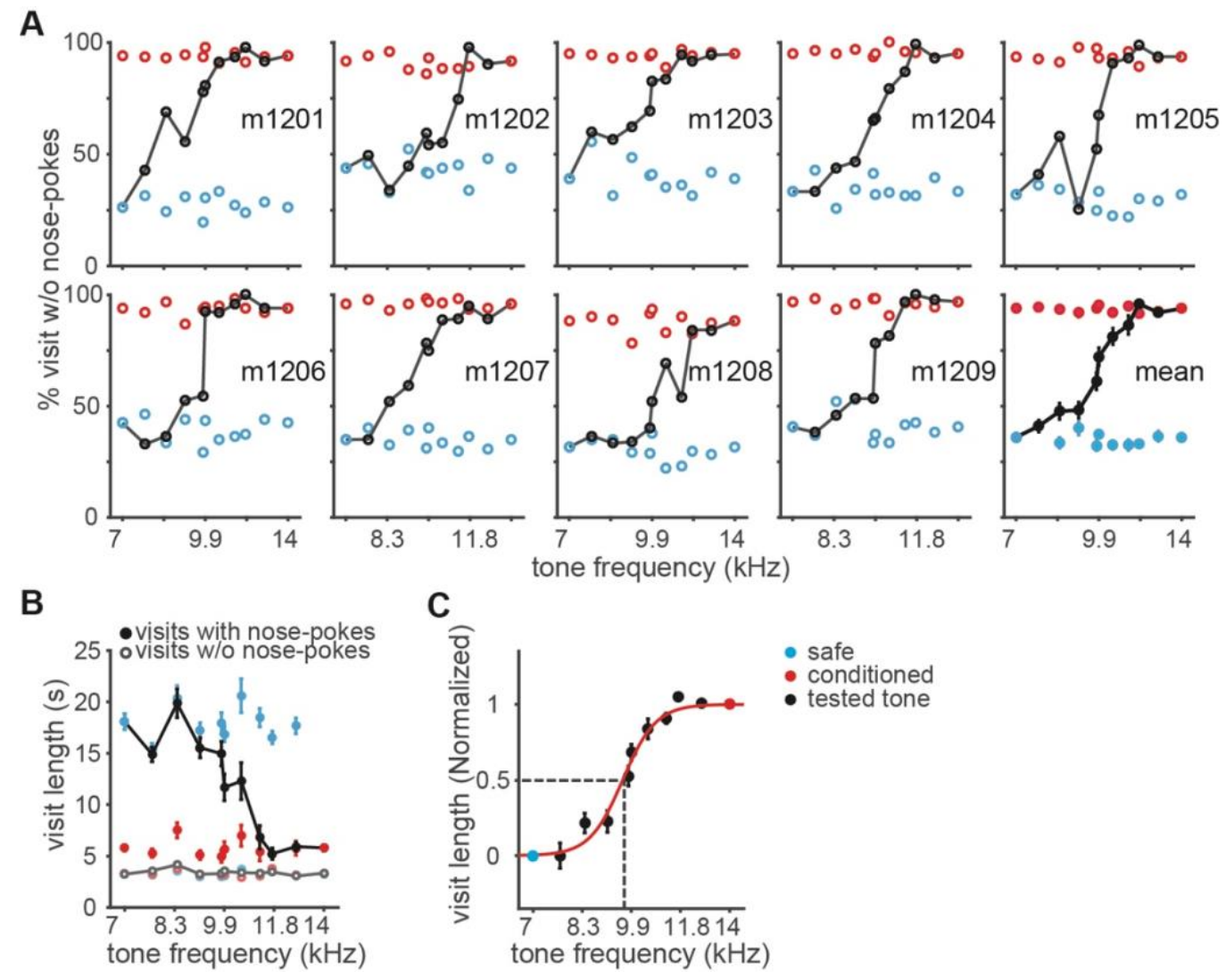

Figure 1-2 Individual performance and visit length analysis

(A) Average response to the safe (blue), conditioned (red) and novel (black) tones as a function of tone frequency for individual mouse. Bottom right: mean responses. (B) Mean visit length of the safe (blue), conditioned (red) and novel (black) visits as a function of tone frequency. Calculation was done separately for visits with (closed dots) and without (open dots) nose-pokes. (C) Normalized inversed visit length (black dots) and fitted psychometric curve (red). Dash line: psychometric threshold or stimulus strength for which performance is at the midpoint.

We estimated the subjective generalization threshold by fitting a logistic function to the normalized psychometric performance and calculated the psychometric threshold, i.e. the relative stimulus distance for which performance is at the midpoint. The psychometric threshold was -0.01, a stimulus level almost equidistant to the safe and conditioned frequencies (Figure 1-1E). This indicates that generalization around the safe and conditioned stimuli was symmetrical.

To understand whether learning and generalization was reflected in other aspects of the behavior other than nose-poking avoidance, we measured the duration of the visit as 
performance index. The average duration of visits in which mice did not nose-poke was relatively short, approximately 3 seconds, independently of whether visits were conditioned, safe, or novel (Figure 1-2B, empty circles). However, for visits in which mice made nose-pokes, the psychometric curve of visit length was again sigmoidal. Conditioned visits with nose-pokes were longer than safe visits with nose-pokes, reflecting the fact that mice were unsure before they made an error and nose-poked (Figure 1-2B, filled circles). The normalized psychometric curve was identical to the one shown before (Figure 1-2C; compare with Figure 1-1E).

\subsubsection{Discrimination task performance deteriorated as the safe-to- conditioned $\Delta \mathrm{F}$ decreased.}

We next investigated how discrimination between positive and negative stimuli was influenced by the physical distance between trained stimuli. The frequency distance between the safe and the conditioned tone was calculated in octaves, defined as $\Delta \mathrm{F}$. We trained different cohorts of naïve mice $(n=77)$ to discriminate between safe and conditioned tones that were $0.25,0.5,0.75$ or 1 octave away from each other (with $4,1,2$ and 3 replications respectively). The safe tone was either $5885 \mathrm{~Hz}$ or $7000 \mathrm{~Hz}$ and the conditioned tone was above the safe tone in frequency. Cohorts have been grouped according to $\Delta \mathrm{F}$ since there was no difference in the behavior for different safe tones. The animals already described above are included within the 1 octave group.

Animals in all groups learnt the task fast and successfully and achieved a significant difference in the nose-poking behavior between safe and conditioned visits in the conditioning phase. To compare the efficiency of learning, we calculated the average percentage of visits without nose-pokes in blocks of 4 visits starting after the first conditioned visit (Figure 1-3A). We did this separately for conditioned visits (red), safe visits that followed a conditioned visit (blue), and safe visits that preceded one (gray). The division of safe visits intended to test the effect of a conditioned visit on the safe ones. When comparing across groups, it became evident that learning to avoid nose-poking in conditioned visits was faster for larger safe-to-conditioned $\Delta \mathrm{Fs}$ and that the conditioning time before meeting the criterion of stable avoidance of the conditioned tone (90\% of visits without nose-poke) increased as the $\Delta \mathrm{F}$ decreased. 


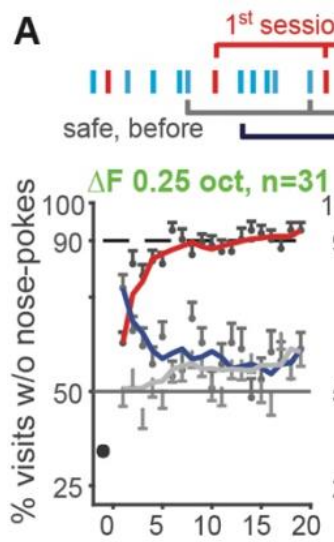

session, conditioning
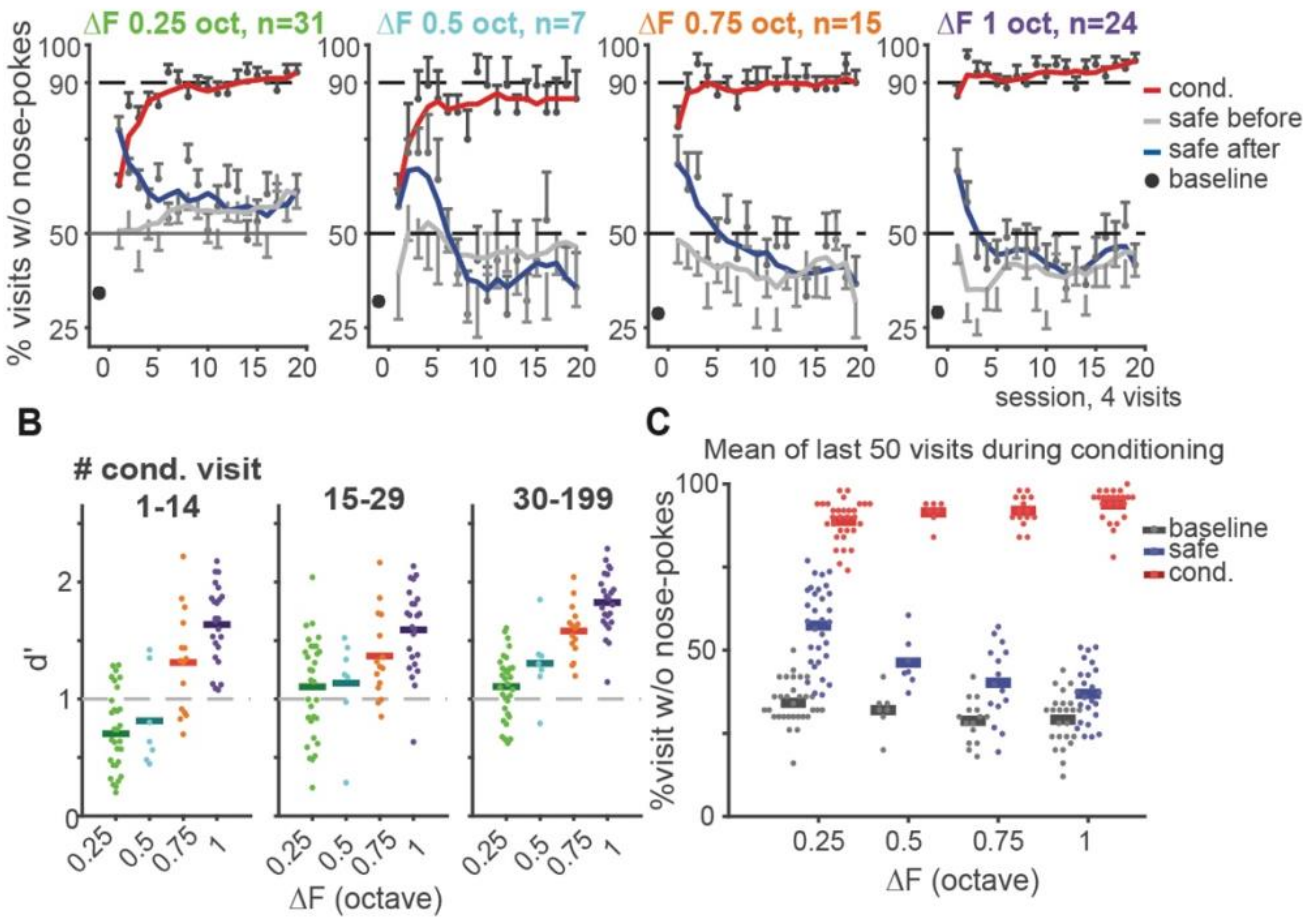

C

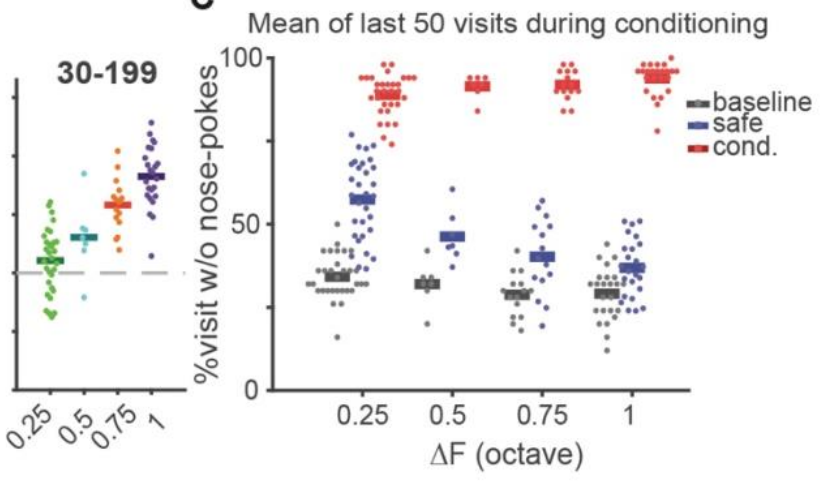

Figure 1-3 Discrimination performance deteriorated as the safe-to-conditioned $\Delta F$ decreased

(A) Top: Schema of learning curve analysis in sessions: average of blocks of 4 visits starting after the first conditioned visit (red) separately for safe visits (blue) that follow conditioned visits (blue brackets), safe visits that precede conditioned visits (gray brackets), and conditioned visits (black brackets). Bottom: performance analysis pooled across groups trained with same safe-toconditioned $\Delta \mathrm{F},(\Delta \mathrm{F} 0.5$ group, 1 replication only). Baseline performance (black dot) calculated as the mean response over the last 50 safe visits before the first conditioned visit. Mean performance in each session was plotted for safe visits following conditioned visits (dark blue), safe visits preceding conditioned visits (gray), and conditioned visits (red). Learning curves were smoothed using a 5 moving average filter. (B) $\mathrm{d}$ ' calculated as a function of the $\Delta \mathrm{F}$ for conditioned visits number 1-14 (left), 15-29 (middle) and 30-199 (right) starting after the first conditioned visit. (C) Mean responses to the safe (blue) and conditioned (red) tones during the last 50 conditioned visits in the conditioning phase across groups trained with different $\Delta \mathrm{Fs}$. Baseline (black) was calculated the same as (A). Here and in subsequent figures, dots in scatter plots represent result for an individual mouse and horizontal bars describe average across animals.

One interesting finding was that discrimination learning was not limited to conditioned visits. The behavior of the mice in the safe visits during the conditioning phase was affected as well. Following each conditioned visit, mice showed increased avoidance of nose-poking in the subsequent safe visit compared with the baseline performance before conditioning began (Figure 1-3A, blue line vs baseline). However, this increase was 
temporary and gradually diminished reaching a plateau after about 20 conditioned visits. In animals trained with the smallest $\Delta \mathrm{F}$, but not the other groups, the plateau remained above baseline throughout (Figure 1-3A, left, gray and blue line).

Thus, as expected, it took longer time for mice trained with smaller $\Delta F$ s to learn to discriminate safe from conditioned sounds. This was reflected in the d' values (Figure 1-3B), a standard measurement of discriminability adopted from signal detection theory, that reflected the separation between the mean of the correct target responses and the false alarms. While animals trained to discriminate across larger $\Delta \mathrm{Fs}$ showed d' values well above 1 already in the first 15 trials, animals trained with smaller $\Delta F s$ required progressively more trials or never achieved this level. This resulted from a combined effect of the speed at which avoidance during conditioned visits was attained and the generalization of this avoidance across to safe visits over slightly longer time-windows, as reflected in Figure 1-3B.

The effect that conditioning had on the safe visits was clear even after overtraining. We quantified animals' steady responses during safe and conditioned visits using the average performance during the last 50 conditioned visits window (Figure 1-3C; ca. last 2 days of conditioning, after 80 to 300 conditioned visits). The baseline performance, which was calculated as the mean performance during the last 50 visits in the safe phase (gray dots), was not significantly different between groups $(p>0.05)$. For both safe and conditioned visits, we observe significant difference in nose-poking behavior between the groups (1way ANOVA of performance on delta $\mathrm{f}$ : for the safe visits $F(3,73)=19.25, p<0.0001$ and for the conditioned visits $F(3,73)=4, p=0.011$; Figure 1-3C). Mice trained with stimuli 0.25 octave apart showed significant lower avoidance in nose-poking in conditioned visits and higher avoidance in the safe visits compared to mice trained with $\Delta \mathrm{F}$ of 1octave. This deterioration in performance was more pronounced for the safe visits compared with conditioned visits ( $29.44 \%$ vs. $4.93 \%$ with respect to baseline).

Notably, since rodent discrimination thresholds lie typically somewhere between $3 \%$ and $6 \% \Delta \mathrm{F}$, all the tested stimulus-pairs were easily discriminable (Clause et al., 2011; Ehret, 1975; Heffner and Masterton, 1980; de Hoz and Nelken, 2014; Syka et al., 1996; Talwar and Gerstein, 1998). Yet, the frequency distance between the conditioned and the safe tone still affected discrimination performance dramatically. This wide-reaching effect of one stimulus over the other was possibly caused by the absolute judgement nature of the task, i.e. the fact that decisions were not based on the comparison between the present sound and the previous one but rather on the present sound alone. These results suggest 
that the dynamics of learning depend mainly on the physical distance between the trained stimuli.

\subsubsection{Shifts in the psychometric threshold with decrease in $\Delta F s$}

The valence associated with a stimulus influences the width of generalization such that often negative stimuli generate more generalization around them than positive stimuli (Schechtman et al., 2010). Here we quantified the effect of the $\Delta \mathrm{F}$ between the safe and conditioned tones on the width of generalization. As before, once mice reached stable discrimination between the safe and conditioned tones, we tested their responses to novel tones. As was the case with the largest $\Delta \mathrm{F}$, during generalization testing mice maintained stable responses to the safe and the conditioned sounds (Figure 1-4B, D, F, blue and red dots respectively). Animals avoided nose-poking during the first visits in which a novel sound was presented (Figure 1-4A, C, E, 1st novel), and later responded to other novel sounds according to their frequency similarities to either the safe or conditioned sound (Figure 1-4B, D, F, testing). 

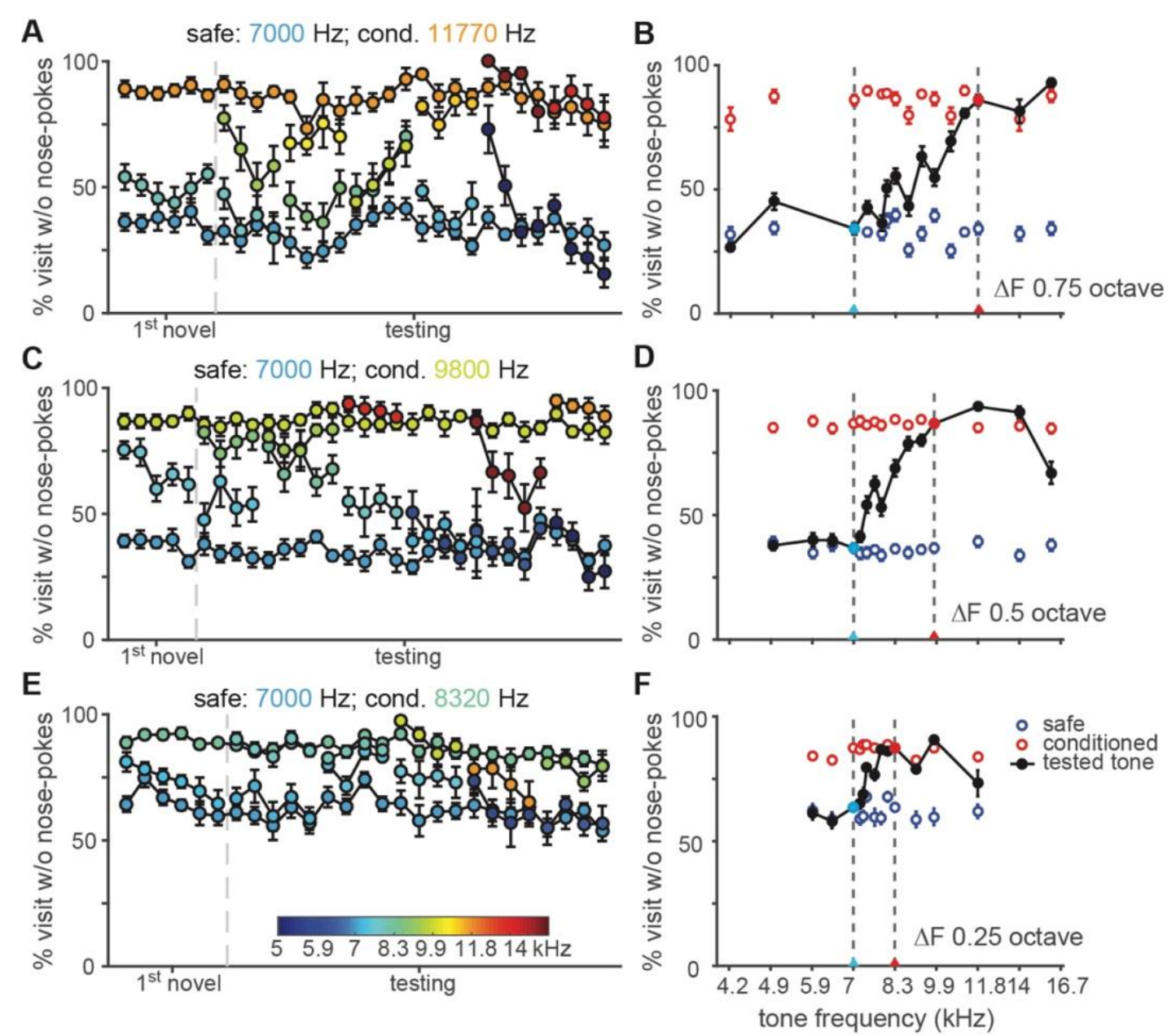

Figure 1-4 Generalization test for mice trained with different $\Delta \mathrm{F}$

(A) Mean daily performance for the safe (blue, $7000 \mathrm{~Hz}$ ), conditioned (orange, $11770 \mathrm{~Hz}$ ), and novel (color based on the frequency) visits during generalization test. Group trained with $\Delta \mathrm{F}$ of 0.75 octaves. (B) Average performance across all visits as a function of tone frequency. Responses to the safe (blue open circles), conditioned (red open circles) tones averaging over testing days of each novel visit type. The triangles on the $\mathrm{x}$-axis indicate the frequency of the safe (blue) and conditioned (red) tone. (C-D) Same as (A-B) for mice trained with $7000 \mathrm{~Hz}$ safe tone and $9800 \mathrm{~Hz}$ conditioned tone. Group trained with $\Delta \mathrm{F}$ of 0.50 octaves. (E-F) Same as (A-B) for mice trained with $7000 \mathrm{~Hz}$ safe tone and $8320 \mathrm{~Hz}$ conditioned tone. Group trained with $\Delta \mathrm{F}$ of 0.25 octaves.

We found that shortening the frequency distance between trained stimuli narrowed the generalization gradient around both safe and conditioned stimuli. This can be seen in Figure 1-5A-B where the flat portion of the psychometric curve becomes shorter as the $\Delta \mathrm{F}$ becomes smaller. There was however a shift in the psychometric curve towards the left (Figure 1-5C) and a decrease in threshold (Figure 1-5D) suggesting that generalization was no longer symmetrical around the safe and conditioned tones, but rather was relatively narrower around the safe tone. No change in slope was observed (Figure 1-5E). 


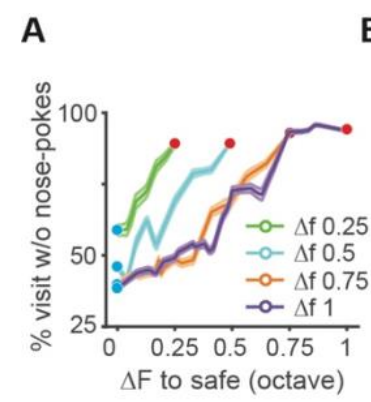

B
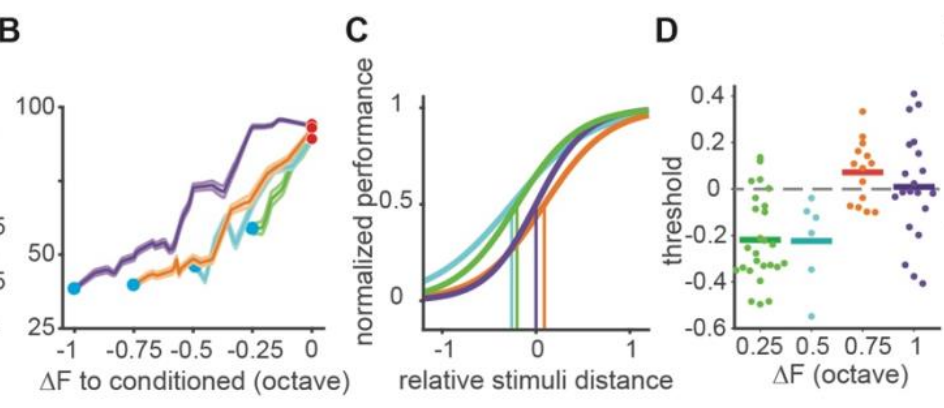

E

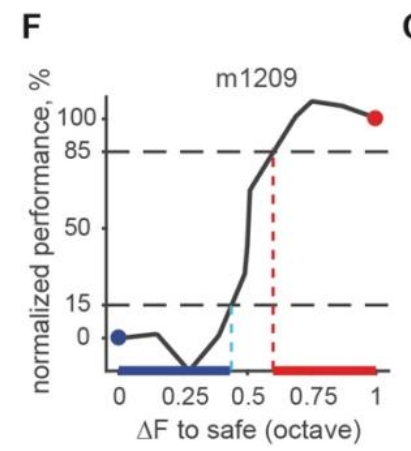

G

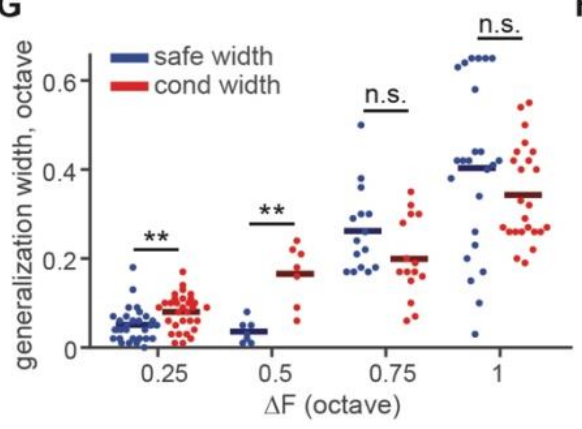

H

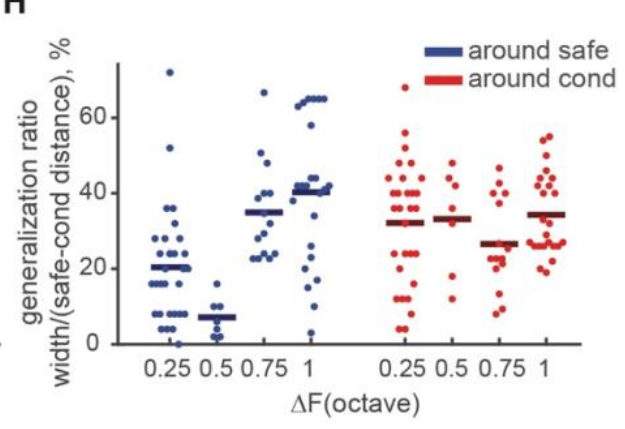

Figure 1-5 Asymmetrical generalization gradients in mice trained with small $\Delta F s$

(A) Average performance as a function of frequency distance to the safe tone across groups trained with different $\Delta$ Fs. (B) Average performance as a function of frequency distance to the conditioned tone across groups trained with different $\Delta \mathrm{Fs}$. (C) Fitted psychometric curves and thresholds (vertical line). (D-E) Individual mouse threshold and slope of the psychometric curves in (C). (A-E) Color coding is based on the $\Delta \mathrm{F}$. (F) Example generalization width around the safe (blue line) and conditioned (red line) tone estimated for one mouse. (G) Generalization width for all mice. Here and in subsequent figures, ${ }^{*} p<0.05,{ }^{*} p<0.01$, ${ }^{* *} p<0.001$, $* * * * p<0.0001$; n.s., not significant $(p>0.05)$. (H) Relative generalization width as percentage of the safe-toconditioned distance for all mice.

To quantify the relative change in generalization, we estimated the generalization width in octaves for individual mice, defined as frequency difference to the safe or conditioned stimuli eliciting more than $15 \%$ change in normalized performance (Figure 1-5F). Corroborating what we saw in the psychometric curves, for both safe and conditioned stimuli mice showed shorter generalization width when the stimuli were physically close. Generalization width around the safe tone and conditioned tone was comparable for safeto-conditioned $\Delta \mathrm{Fs}$ above 0.5 octave. Smaller $\Delta \mathrm{Fs}$, however, led to significantly wider generalization around the conditioned stimuli than around the safe stimuli (Figure 1-5G). However, when generalization width was measured, not in octaves, but as a percentage of the safe-to-conditioned tone distance, the pattern changed (Figure 1-5H). The relative generalization width around the conditioned sound was constant across $\Delta F$ s with a value of about $30 \%$ (1-way ANOVA of relative generalization width on $\Delta \mathrm{F}: F(3,73)=1.04, p=$ 
0.38). However, the relative generalization width around the safe sound was significantly lower for mice trained with $\Delta F$ s below 0.5 octave (1-way ANOVA of relative generalization width on delta f: $F(3,73)=12.79, p<0.0001)$. Thus, a decrease in the safeto-conditioned $\Delta \mathrm{F}$ does not have an effect on the relative generalization around the conditioned sound, nor on the slope of the generalization but it has an effect on the level of generalization around the safe sound.

Generalization was not limited to frequencies in between the safe and conditioned tones, but also happened for flanking frequencies which were either below the safe tone or above the conditioned tone. Within the range of the frequencies tested, animals showed similar performance in response to the flanking frequencies close to the safe tone independently of which $\Delta \mathrm{F}$ was used (Figure 1-4B, D, F). However, we observed, as others have done before (Ghirlanda and Enquist, 2003; Verzijden et al., 2007), a shift in the peak of the generalization gradient for the conditioned tone for mice trained with a $\Delta \mathrm{F}$ of less than 1 octave. As shown in Figure 1-4, the avoidance response was strongest at the conditioned tone for mice trained with $\Delta \mathrm{F}$ of 1 octave, but was displaced from the conditioned tone away from the safe tone for other groups. This is further indication that an interaction between the safe and the conditioned tone happened only for lower $\Delta \mathrm{Fs}$.

\subsubsection{Neuroligin 2 knockout mice showed impaired discrimination performance but normal generalization gradient.}

There are two main possible reasons for the narrower generalization around the safe sound for smaller safe-to-conditioned $\Delta F$ s: it can be a result of an increased valence interaction per se; or it can be a byproduct of increased anxiety, i.e. stressed mice tend to be more cautious (Dibbets and Evers, 2017; Laufer et al., 2016). If anxiety leads to wider generalization around the negative stimulus than around the positive one, increasing the anxiety level in mice trained with a large $\Delta \mathrm{F}$ (e.g. 1 octave) should elicit a shift in the psychometric threshold.

Therefore, we tested generalization in Neuroligin 2 knockout (KO) mice, as a model of anxiety. Neuroligin 2 (Nlgn2) is a synaptic adhesion protein that is thought to be exclusively localized at inhibitory synapses (Varoqueaux et al., 2004). The loss of function of Nlgn2 in mice leads to relative selective increase in behavioral anxiety (Babaev et al., 2016; Blundell et al., 2009). In this experiment, the safe sound was $7000 \mathrm{~Hz}$ and the conditioned, $14000 \mathrm{~Hz}$. Nlgn2 KO mice showed comparable baseline behavior compared 
to WT littermates (Figure 1-6A-B, baseline). Unexpectedly though, when conditioning began, Nlgn2 KO mice showed less avoidance during conditioning visits than WT animals (Figure 1-6A-B, red line). Interestingly, the effect that the first two dozen conditioned visits had on subsequent safe visits, reflected in the increase in avoidance during safe visits following conditioned visits (see blue line in Figure 1-6A and, as previously described, Figure 1-3A), was absent in the Nlgn2 KO mice. The overall effect was a reduction in d' in these mice, which was below 1 throughout the time of testing (Figure 1-6C; 2-way ANOVA of d' on training days and genotype revealed main effects on training days, $F(7,135)=3.61, p=0.0014$; and main effects on genotype, $F(1,135)=101.96, p=0)$. But while the decrease in $d^{\prime}$ in animals trained with small $\Delta \mathrm{Fs}$ was a result of an increase in avoidance during safe visits, in the Nlgn2 $\mathrm{KO}$ mice it was caused by a decrease in avoidance during conditioned visits $(92.98 \%$ avoidance for the WT, $72.26 \%$ for Nlgn2 KO; unpaired t-test for the mean performance during the last 3 days of conditioning, $p<$ $0.0001)$.
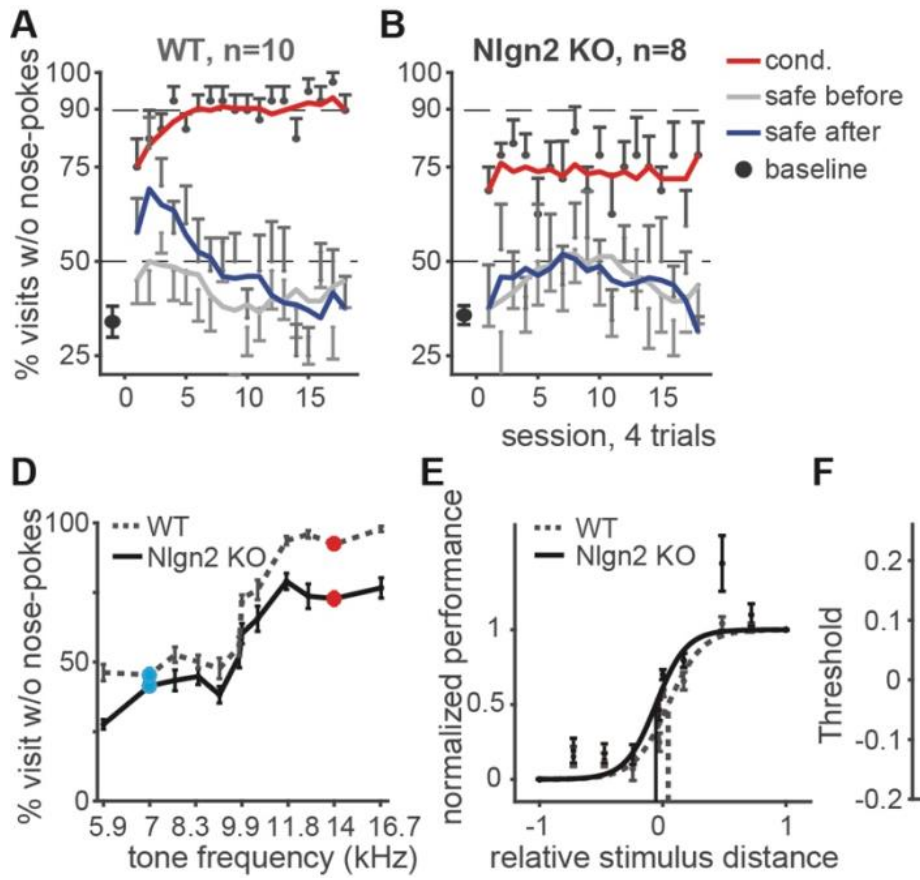

C

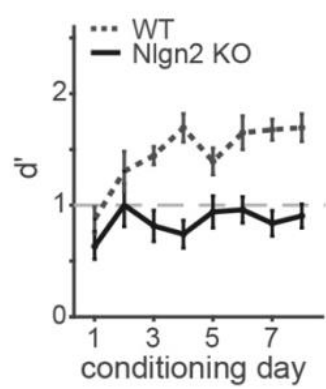

G
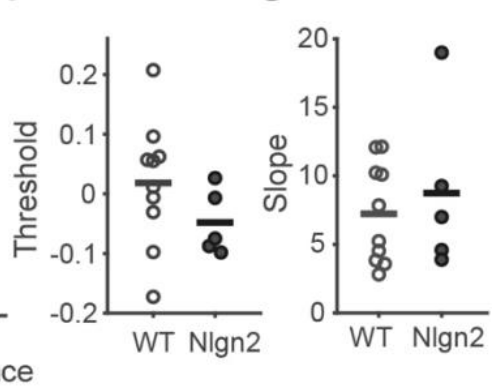

Figure 1-6 Neuroligin 2 knockout mice showed impaired discrimination but normal generalization gradients

(A) Baseline performance (black dot) and learning curves for Nlgn2 WT mice. (B) Same as (A) for Nlgn2 KO mice. (C-E) Comparison between Nlgn2 WT (gray dash line) and KO (black solid line) mice. (C) Mean d' calculated as a function of conditioning day. (D) Generalization gradients based on average performance as a function of visit tone-frequency. (E) Fitted psychometric curves and thresholds (vertical line). (F-G) Threshold and slope of the psychometric curves, respectively. Mice did not pass the goodness of fit were excluded in the individual plot. 
This was not the result of increased generalization around the safe tone since the shape of the psychometric curves in Nlgn2 KO and WT mice was very similar despite the very different end points (Figure 1-6D; full two-way ANOVA model revealed a significant effect of genotype (Nlgn2 KO vs WT, $p=0.0005)$, significant effect of stimuli $(F(9,140)=$ 79.61, $p=0)$, and no interaction $(p=0.17)$ ). There was no significant difference in the psychometric threshold between the groups, which was equidistant from the safe and conditioned tones (Figure 1-6E-F; unpaired t-test, $p=0.21$; average threshold for Nlgn2 $\mathrm{KO}$ is -0.048; for WT is 0.018). This indicates that, like in the WT, the Nlgn2 KO mice generalized equally around the safe and conditioned tone and that the width of the generalization was similar to that of WT animals. There was also no difference in the slope of the psychometric curve between the groups (Figure 1-6G; $p=0.56$ ).

Thus, Nlgn2 KO mice showed impaired discrimination during learning and yet both the slope and width of the generalization gradient was comparable to that of WTs, indicating that while valence assignment in these mice is affected, sensory acuity is not. These results indicate that the width of the generalization gradient is not a byproduct of the anxiety level of the animal and confirm the results described above that the width of generalization depends on the physical distance between the to-be-discriminated stimuli.

\subsubsection{The direction of conditioning along the frequency axis influences discrimination learning but not generalization}

Cells in the auditory system often have asymmetrical tuning curves, with shallower slopes for frequencies below that which elicits the strongest response (Egorova et al., 2006). Potentially, this asymmetrical tuning could have an effect on discrimination learning and generalization (Bang et al., 2008). Since the conditioned tone was above the safe tone in the experiments described so far, the shallower tuning towards the safe tone might have affected the pattern of results. We now trained three groups of mice with $\Delta \mathrm{F}$ of either 0.75 or 1 octave in the Audiobox as we did before but using the low frequency tone as conditioned and measured avoidance during conditioned visits as well as during safe visits before and after conditioning began (Figure 1-7A-C; right data are replotted from the subset in Figure 1-3C which was trained with corresponding frequencies). A comparison with data described before, confirmed that mice showed slightly better discrimination in tasks where high frequency tone was conditioned (Figure 1-7D; overall unpaired t-test, $p<$ 0.0001). Combining all groups, a 3-way ANOVA revealed a significant main effect of 
conditioning $(F(1,100)=765.46, p=0)$, a main effect of whether conditioned tone was higher than the safe one $(F(1,100)=11.96, p=0.0008)$, and a significant interaction between the two $(F(1,100)=10.93, p=0.0013)$ but no effect of $\Delta \mathrm{F}(F(1,100)=1.76, p=$ $0.19)$.
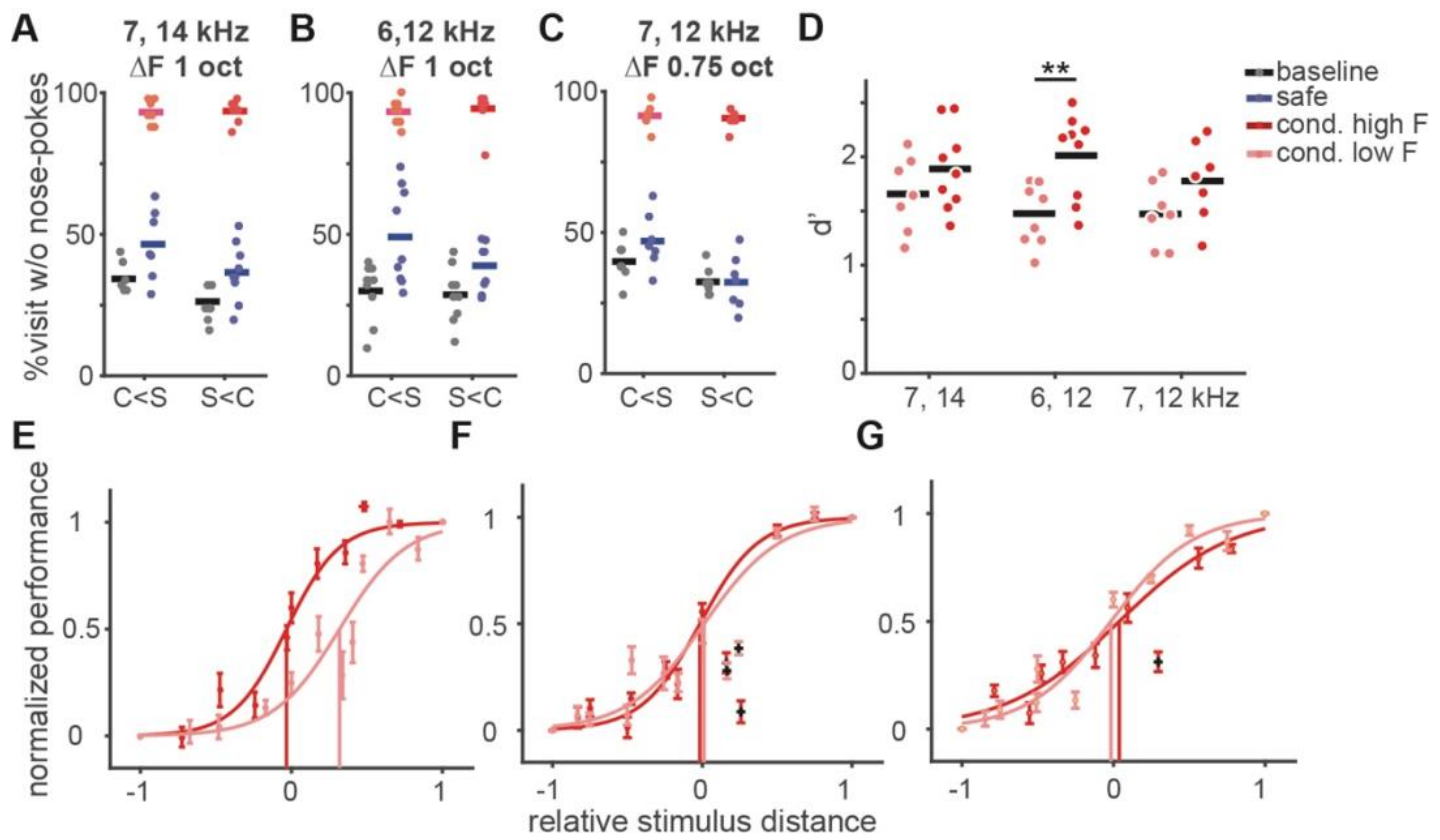

Figure 1-7 The direction of conditioning along the frequency axis affected learning, but not generalization

(A-C) Comparison between mice trained with different direction of conditioning (conditioned tone was higher or lower than safe) along the frequency axis. Baseline (black) and average responses to the safe (blue) and conditioned (pink/red) tones during the last 50 conditioned visits in the conditioning phase. All mice in a plot were trained with the same pair of stimuli, (A) $7000 \mathrm{~Hz}$ and $14000 \mathrm{~Hz}$ tone, (B) $5885 \mathrm{~Hz}$ and $11770 \mathrm{~Hz}$ tone, and (C) $7000 \mathrm{~Hz}$ and $11770 \mathrm{~Hz}$ tone. (D) Mean d' during the last 50 conditioned visits in the conditioning phase. (E-G) Fitted psychometric curves and thresholds (vertical line) for mice described in (A-C), respectively. Black dots indicate the outliers in the fitting.

Generalization on the other hand was overall more invariant (Figure 1-7E-G). Only in one of the three groups, trained with 1 octave $\Delta \mathrm{F}$ (safe $=14000 \mathrm{~Hz}$ and conditioned $=7000$ $\mathrm{Hz}$ ), we found a significant shift in the psychometric threshold towards the conditioned sound compared to the opposite group (Figure 1-7E).

These findings suggest that discrimination learning is influenced by both the $\Delta \mathrm{F}$ and the polarization, whereas for generalization only the first one matters. These data support again the idea that generalization gradients depend strongly on the physical distance of the trained stimuli. 


\subsubsection{Previous experience with the task did not facilitate discrimination but shifted generalization gradients}

We investigated how previous experience in the Audiobox influenced learning of new conditioned sounds, existing generalization gradients, and discrimination acuity. After training and testing animals with one pair of safe and conditioned tones, we replaced the initial conditioned tone for a novel conditioned tone, without changing the safe tone. Mice were then trained with only the safe and new conditioned tone for a few days before generalization curves with tones in between were measured. In this second phase of generalization testing the first conditioned tone was not conditioned anymore (Figure $1-8 \mathrm{~A})$.

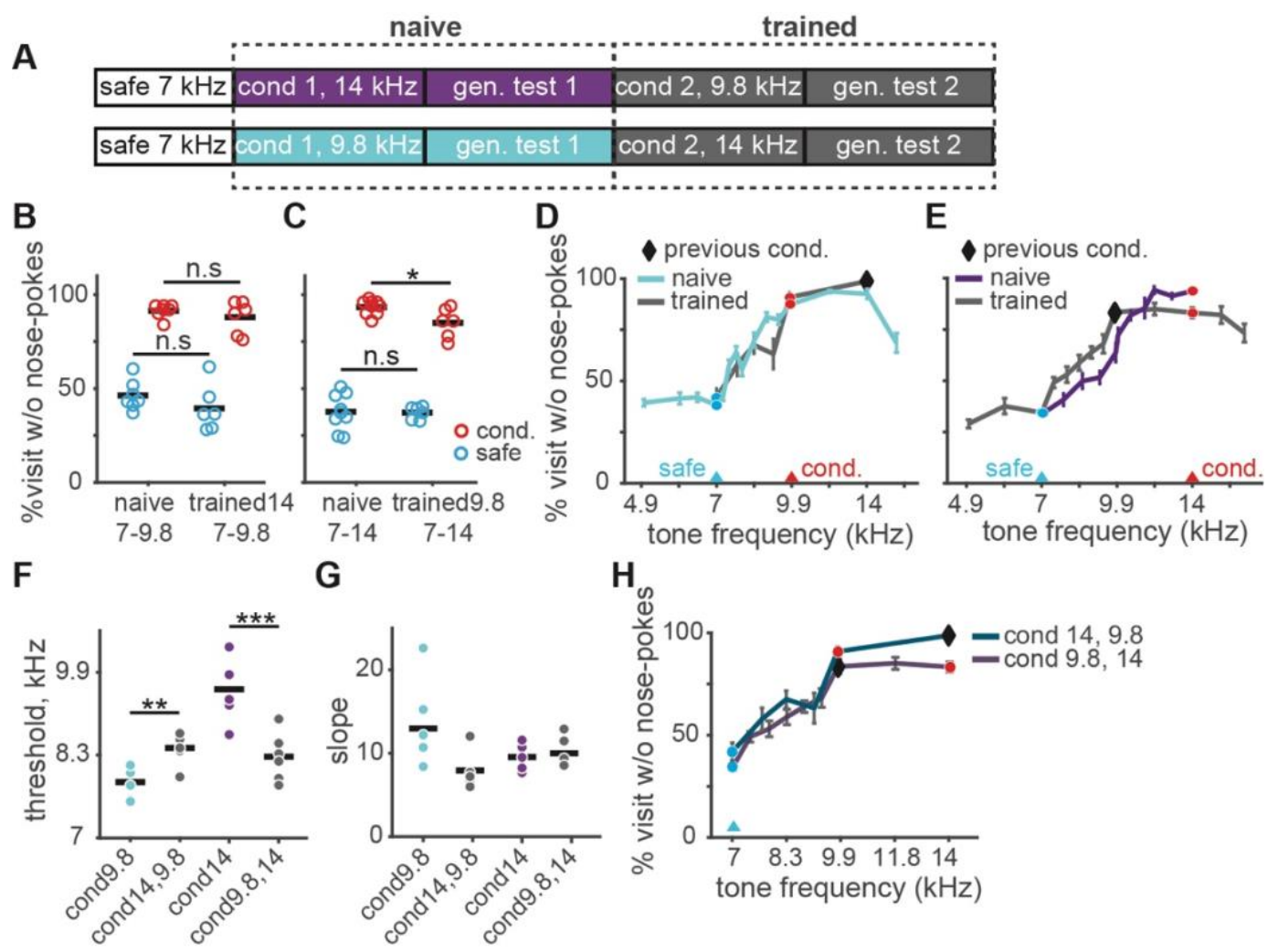

Figure 1-8 Previous task experience did not facilitate discrimination but shifted generalization gradients

(A) Experimental design: two groups of mice were initially conditioned with $14000 \mathrm{~Hz}$ or $9800 \mathrm{~Hz}$ tone, and then retrained to $9800 \mathrm{~Hz}$ or $14000 \mathrm{~Hz}$ tone, respectively. The safe tone remained at 7000 $\mathrm{Hz}$ throughout. Generalization gradients were measured following each conditioning. (B) Comparison of discrimination performance between naïve ( $1_{\text {st }}$ conditioning) and trained ( 2 nd conditioning) mice conditioned to $9800 \mathrm{~Hz}$ tone. Average responses to the safe (edge color blue) and conditioned (edge color red) tones during the last 50 conditioned visits in the conditioning phase. (C) Same as (B) for naïve and trained mice when conditioned to $14000 \mathrm{~Hz}$ tone. (D) 
Generalization gradients for naïve and trained mice following conditioning to a $9800 \mathrm{~Hz}$ tone. (E) Same as (D) for mice following conditioning to a $14000 \mathrm{~Hz}$ tone. (F-G) Threshold and slope of the psychometric curve for all mice in each testing. (H) Comparison of generalization gradients between mice conditioned to $14000 \mathrm{~Hz}$ then $9800 \mathrm{~Hz}$ and those conditioned to $9800 \mathrm{~Hz}$ then 14000 Hz. (D, E, H) The safe (blue circle), conditioned (red circle), as well as previously conditioned tone (black diamond) was marked respectively.

First, we compared the performance of mice that had been trained with a $\Delta \mathrm{F}$ of 1 and 0.5 octaves before being retrained with, respectively, a $\Delta \mathrm{F}$ of either 0.5 or 1 octave (Figure $1-8 \mathrm{~B}-\mathrm{C}$, right), with the performance of naïve mice trained directly with a $\Delta \mathrm{F}$ of either 0.5 or 1 octave (Figure 1-8B-C, left). Mice in all groups showed similar responses to the safe tone (unpaired t-test, $p>0.05$ for all groups). Interestingly, despite their different training history, mice first conditioned with an easier high $\Delta \mathrm{F}$ (1 octave; Figure 1-8B right) did not facilitate discrimination relative to naïve animals when further conditioned with a lower $\Delta \mathrm{F}(0.5$ octave; Figure $1-8 \mathrm{~B})$. This suggests that there is no knowledge transfer from an easy to a hard discrimination. When we first trained mice with a more difficult low $\Delta \mathrm{F}(0.5$ octave), however, there was also no facilitation of a subsequent discrimination with a high $\Delta \mathrm{F}$ (1 octave, Figure 1-8C right) relative to naïve mice (Figure 1-8C left). Performance was, in fact, subtly but significantly worse (unpaired t-test, $p=0.016$ ). Overall the data suggest that previous training did not facilitate discrimination learning but rather set the maximum level of avoidance.

We then investigated how generalization was shaped by previous experience. We compared the generalization gradients of naïve and experienced mice and measured the psychometric threshold (Figure 1-8D-F). The tone that was first conditioned is marked in the generalization gradient but is no longer conditioned during this second generalization testing. In Figure 1-8D-E it is evident that previous training has an effect on the generalization gradient, which is subtly different from that in naïve mice. Indeed, the psychometric threshold, measured here in frequency values rather than relative distance as before, was significantly different from that of naïve mice (Figure 1-8F; unpaired t-test, conditioned at $9.8 \mathrm{~Hz}, p=0.006$; conditioned at $14 \mathrm{kHz}, p=0.0002)$. There was no significant difference in slope (Figure 1-8G). Interestingly, the order of conditioning did not influence generalization gradient (Figure 1-8H). Animals conditioned with $14 \mathrm{kHz}$ tone followed by $9.8 \mathrm{kHz}$ showed similar psychometric threshold than animals trained in the reversed order, first $9.8 \mathrm{kHz}$ then $14 \mathrm{kHz}$ (unpaired t-test, $p<0.05$ ). This indicates that 
even though previous experience did not lead to better discrimination, it elicited shifts in the generalization gradient. Generalization is a summation of the animals training history.

We then compared the first and second generalization gradient for the same animals after the first and second conditioning respectively. More groups were included in this analysis, with $\Delta \mathrm{F}$ ranging from 0.125 to 1 octave. In all groups, we found that retraining shifted generalization gradients towards the second conditioned frequency (Figure 1-9A-E, leftmost column). This effect was especially strong in animals that were initially trained with $\Delta \mathrm{F}$ above half an octave and retrained to a lower $\Delta \mathrm{F}$ (Figure 1-9A, C, D, middle column; comparing thresholds; paired t-test, $\left.p_{s 7 A}=0.007, p_{s 7 C}=0.0005, p_{s 7 D}=0.005\right)$. For mice first trained with relatively small $\Delta \mathrm{F} 0.25$ octave and retrained to a smaller $\Delta \mathrm{F}, 0.125$ octave, their psychometric thresholds tended to shift but without statistical significance (Figure 1-9E, middle column; paired t-test, $p=0.15$ ). Conditioning the mice to a higher $\Delta \mathrm{F}$ than the first conditioning also moved the psychometric threshold away from the safe tone but with no statistical significance (Figure 1-9B, middle column; paired t-test, $p=0.053$ ). For all groups, we found that there was no consistent change in slope (Figure 1-9, rightmost column; paired t-test, $p_{A}=0.57, p_{B}=0.16, p_{C}=0.16, p_{D}=0.81, p_{E}=0.33$ ). These results confirmed that generalization gradients for animals trained with multiple conditioned sounds reflects the history of training and not simply the level of avoidance of the last conditioned tone. 


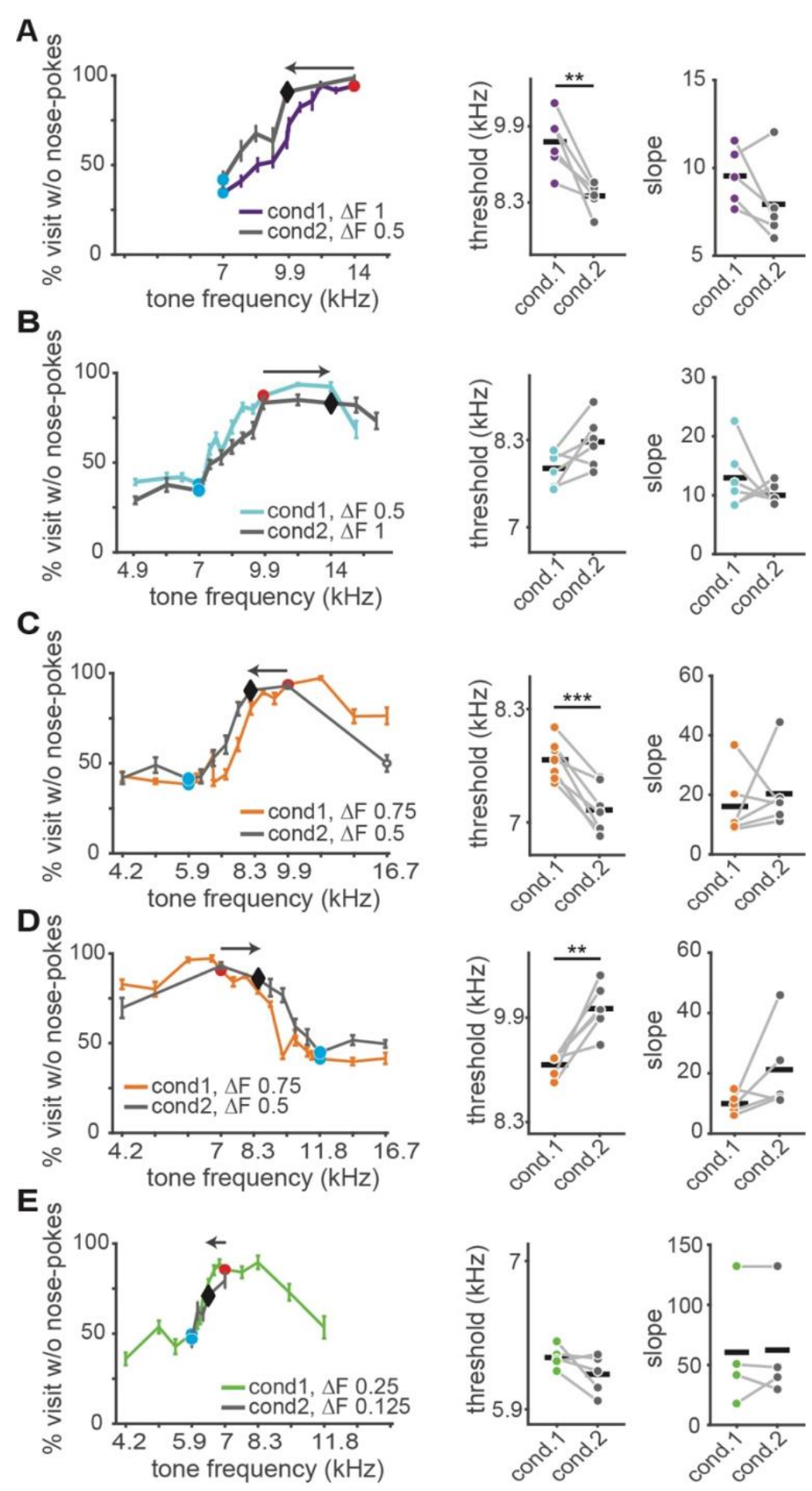

Figure 1-9 Retraining to another conditioned tone shifted psychometric threshold

(A-left) Average generalization gradients following the first (purple; $\Delta \mathrm{F}$ 1octave) and the second (gray; $\Delta \mathrm{F} 0.5$ octave) conditioning. (A-middle and right) Threshold and slope calculated from fitted the psychometric curve. (B-E) Same as (A) for the remaining groups. The safe (open circle) and conditioned (closed circle) tone used in each conditioning was marked respectively. The gray arrow indicates the direction in which the second conditioned tone moved away from the first one. The safe-to-conditioned $\Delta \mathrm{F}$ for each generalization gradient was shown in the label. 


\subsubsection{Discrimination acuity was increased around the conditioned tone after Audiobox learning in a $\Delta \mathrm{F}$-specific manner.}

In order to test whether discrimination training in the Audiobox led to changes in sensory perception we tested acuity around both the conditioned and safe tones. To avoid task-specific influences we tested acuity using the pre-pulse inhibition (PPI) of the acoustic startle response (ASR) protocol. We aimed to understand, for example, whether the narrowing in the generalization gradient observed in animals trained with a small $\Delta \mathrm{F}$ was a result of a change in discrimination acuity per se. In the PPI protocol, ASR can be partially inhibited by a preceding warning pre-pulse tone. The inhibition effect of the pre-pulse tone on ASR magnitude is highly depended on the saliency of this tone. If a constant background tone is present, the disparity in frequency between the background and the prepulse tone will determine the efficacy of the latter in inhibiting the ASR (see Methods, Figure 0-2A-B; (Aizenberg and Neimark Geffen, 2013; Basavaraj and Yan, 2012; Clause et al., 2011)). Discrimination acuity was quantified in terms of frequency discrimination threshold, defined as the difference between background and pre-pulse frequency that generated $50 \%$ of the maximum inhibition (see Methods, Figure 0-2C). Since there are innate differences in discrimination acuity for different frequencies, we focused on the change in discrimination thresholds triggered by training (before versus after) and tested individual animals using either the Audiobox safe or conditioned tones as the background (f1, constant across the two PPI tests for a given animal). We focused on groups with safeto-conditioned $\Delta \mathrm{F}$ of 0.25 and 1 octave, which were trained with $7 \mathrm{kHz}$ as safe and either $8.32 \mathrm{kHz}$ or $14 \mathrm{kHz}$ as conditioned.

Training led to different effects on discrimination acuity around the safe and conditioned sounds. Animals in both groups, showed a significant increase in inhibition that was surprisingly specific for the tone 0.03 octave above the safe tone (Figure 1-10A-B, middle). The overall discrimination threshold around the safe tone tended to decrease, but without statistical significance (Figure 1-10C-D, 7-up; paired t-test, $p=0.17$ ). Thus, training tends to improve discrimination acuity around the safe tone, but only for frequencies above this tone (towards the conditioning tone). Animals in the $0.25 \Delta \mathrm{F}$ group showed a non-significant tendency for decreased PPI for pre-pulse tones around the 8.32 $\mathrm{kHz}$ conditioned tone (Figure 1-10A, bottom) and increased threshold (Figure 1-10C, 8down; paired t-test, $p=0.10$ ). The strongest effect was seen for pre-pulse tones when the $14 \mathrm{kHz}$ conditioned tone was used as background in the 1 octave group. Following 
Audiobox discrimination training PPI was significantly enhanced for pre-pulse tones both above and below the $14 \mathrm{kHz}$ conditioned tone (Figure 1-10B, bottom). The result was a significant decrease in threshold around $14 \mathrm{kHz}$ that was more pronounced for pre-pulse tones below the conditioned tone (towards the safe tone; Figure 1-10D, 14-down; paired ttest, $p=0.03)$.

A
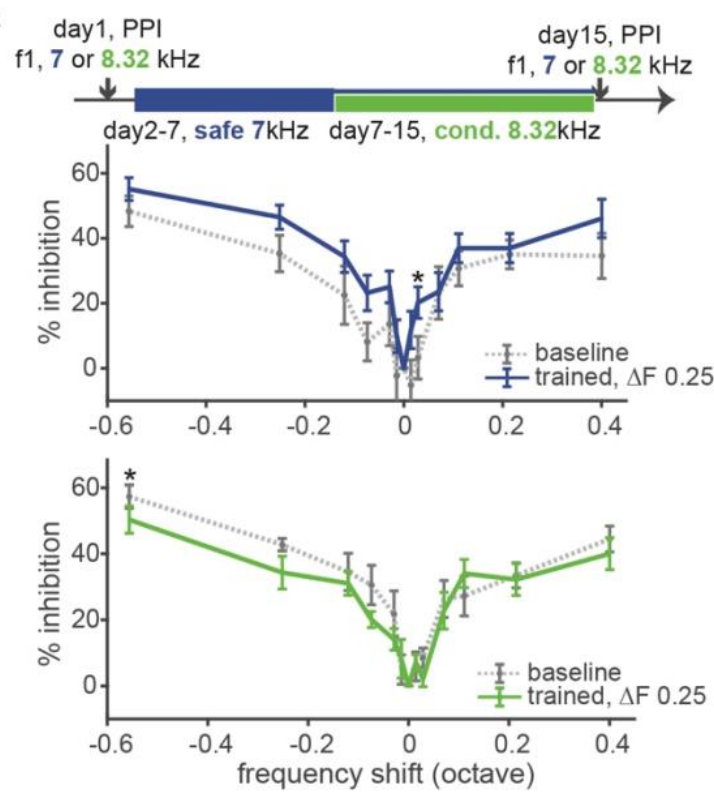

C

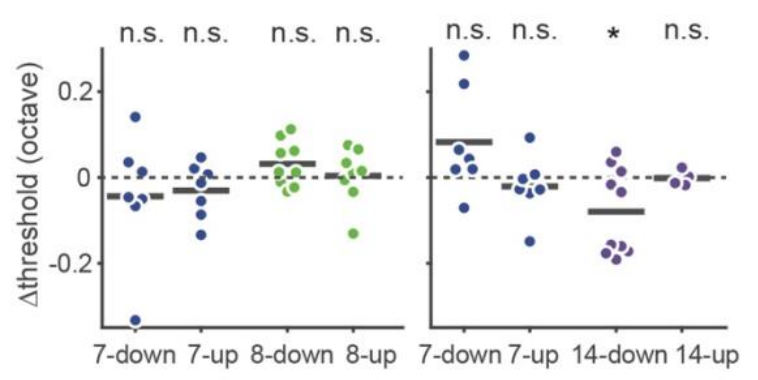

$E$
B
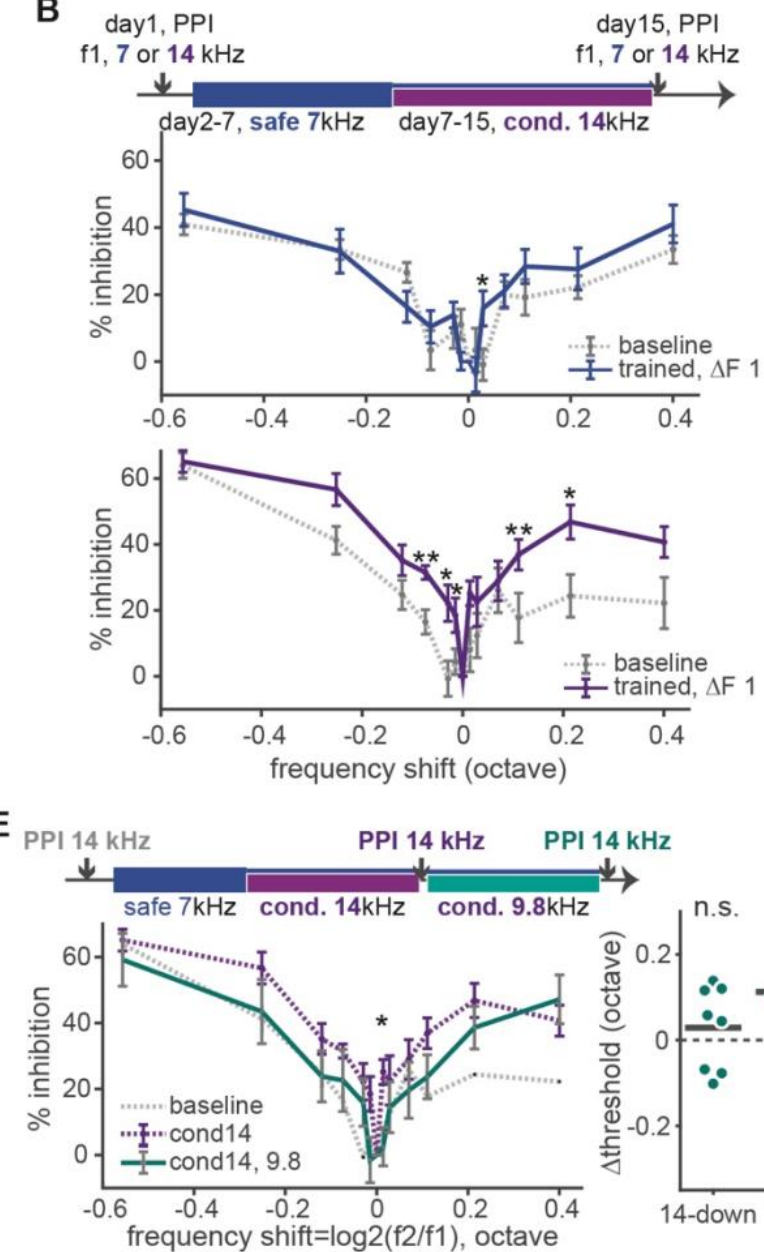

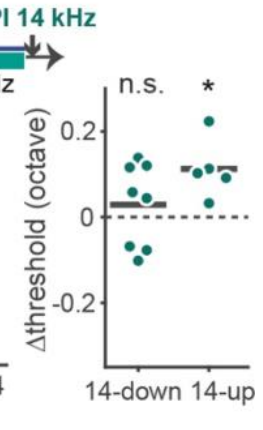

Figure 1-10 Audiobox learning led to $\Delta \mathrm{F}$-specific increase in discrimination acuity around the conditioned tone

(A-top) Experimental design: mice were tested for PPI-based discrimination acuity both before and after the Audiobox training. Two groups of mice were tested separately using either the safe or the conditioned tone as the background sound in both PPI tests. (A-middle) Baseline PPI (gray dash line) and PPI following Audiobox training (blue solid line) as a function of frequency shift from background (f1) where $\mathrm{f} 1$ was the same frequency as the safe tone $(7000 \mathrm{~Hz})$. (A-bottom) Baseline PPI (gray dash line) and PPI following Audiobox training (green solid line) as a function of frequency shift from $\mathrm{f} 1$ where $\mathrm{f} 1$ was the frequency as the conditioned tone $(8320 \mathrm{~Hz})$. (B) Same as (A) for mice conditioned to $14000 \mathrm{~Hz}$. (C) Change in discrimination threshold around the safe $(7000 \mathrm{~Hz})$ and conditioned $(8320 \mathrm{~Hz})$ tone following Audiobox training. Calculation was done separately for $\mathrm{f} 2$ lower (down) and higher (up) in frequency than the background tone. (D) Same as (C) for mice trained with $7000 \mathrm{~Hz}$ and $14000 \mathrm{~Hz}$ tone. (E-top) Experimental design: mice went through two conditioning and PPI was tested before and after each conditioning using $14000 \mathrm{~Hz}$ as 
background tone throughout. (E-bottom left) Baseline PPI (gray dash line) and PPI following the first (purple dash line) and second (cyan solid line) conditioning as a function of frequency shift when $\mathrm{f} 1$ was the same in frequency as the first conditioned tone $(14000 \mathrm{~Hz})$. (E-bottom right) Change in Discrimination threshold around the first conditioned $(14000 \mathrm{~Hz})$ tone following the second conditioning.

We then investigated how subsequent conditioning affected the change in acuity induced by the initial conditioning (Figure 1-10E, top). For mice that were initially conditioned with a $14 \mathrm{kHz}$ tone and subsequently conditioned with a $9.8 \mathrm{kHz}$ tone, we found that the effect of initial conditioning on acuity was partially reversed by the second conditioning (Figure 1-10E-F). This suggests that the modulation of acuity induced by learning is very dynamic.

Taken together, wider generalization gradients in the Audiobox were not the result of diminished perceptual discrimination. On the contrary and paradoxically, in the high $\Delta \mathrm{F}$ group, the wider generalization observed in the Audiobox was accompanied by increased discrimination acuity. In turn, narrower generalization in the low $\Delta \mathrm{F}$ group was not accompanied by improved acuity.

\subsection{Discussion}

In the present study, we assessed learning and generalization in C57BL/6J mice in a tone frequency discrimination task in an automatic and naturalistic environment, the Audiobox. The task required the mice to make memory-based decisions and had the characteristics of a go/no-go discrimination task. We investigated how (1) the frequency distance between trained stimuli, and (2) the mice past experience with these stimuli, affected leaning speed, discrimination performance and generalization gradients. First, we found that the physical distance between stimuli, the difference in frequency between the safe and conditioned sounds, was the main contributor to discrimination performance while the past experience with the same sounds exerted a weaker influence. Second, we found that while the generalization slope was constant throughout the different manipulations, generalization width, was influenced both by the physical distance between the to-bediscriminated tones and the mice past experience. Third, valence had a modulatory influence on the generalization width only when the distance between the safe and conditioned sounds was decreased such that task difficulty was increased. In this case, although generalization was overall narrower, it became asymmetrically wider around the 
conditioned sound. Fourth, generalization around a sound did not reflect a decrease in sensory acuity around the same sound.

In conclusion, the tonotopic organization of the auditory system in mice is the main determinant of discriminative task performance in the auditory processing. In relative judgement tasks, where discrimination is based on the difference between the current stimulus and the one immediately preceding, the history of activation of the involved neuronal populations is likely to have a strong impact on discrimination. Memory-based discriminations might be inherently harder (Smith and Schmuckler, 2008) because the pattern of activity triggered by, say, the conditioned tone cannot be directly compared to the pattern of activity triggered by the presentation of the safe tone. Based on our results, we conclude that performance in memory-based discriminations relies on wide sensory filters. One possible explanation is a strong influence of the wide tuning typical of subcortical structures (Egorova et al., 2006; Taberner and Liberman, 2005).

\subsubsection{Naturalistic behavioral paradigm}

In our study, we reliably measured leaning speed, discrimination performance and generalization gradients through an Audiobox paradigm that mimics the natural environment to the extent that it is possible in a well-controlled experimental setting. As mentioned in a previous study (de Hoz and Nelken, 2014), the Audiobox allows training and testing in the animal's living quarters. Mice live for days at a time in groups of 8 to 10 subjects and are neither food-nor water- deprived, reproducing the social environment that is natural for rodents. Moreover, the automatic detection system (see methods) allows mice to initiate stimulus exposure at will, unlike in other operant protocols (Jaramillo and Zador, 2011; Kurt and Ehret, 2010). Task attendance was driven by innate curiosity and water demand, allowing us to simplify the procedural learning. Overall, this led to fast learning and stable performance.

The animal's innate curiosity results in a high number of corner visits per day (on average 110 during conditioning and 150 during generalization phases). As a result, there is a high false alarm rate: in about one third of safe visits mice do not nose-poke. Performance was quantified using nose-poking behavior, but visit duration correlates well with nose-poking, with the shortest visits (about 3 seconds) being those that are accompanied by the conditioned sound. 


\subsubsection{The physiological constrain is prioritized in perception}

The present data show that learning speed, discrimination performance, as well as generalization gradients are dependent on the physical distance between the trained stimuli. Lower safe-to-conditioned $\Delta \mathrm{F}$ elicited slower learning, worse discrimination and narrower generalization gradients around trained stimuli. Just-noticeable differences in mice were previously reported to be around 2-5\% in relative judgement tasks (Clause et al., 2011; Ehret, 1975; Heffner and Masterton, 1980) or 4-7\% in a comparable absolute judgement task (de Hoz and Nelken, 2014), all well below the $\Delta$ Fs used here. Had the main constrain in our task been the discriminability of the sounds, we would have expected discrimination performance to remain at high-level and only drop, relatively, suddenly, when the $\Delta \mathrm{F}$ was near the JND. What we found, however, is that even as the $\Delta \mathrm{F}$ decreased from 1 octave (100\%) to 0.25 octave (19\%), both well above the JND, discrimination performance deteriorated dramatically. This could mean that the internal representation along the frequency axis of the safe and conditioned sounds, which is probably the basis of absolute judgement discriminations, interact with each other in ways that influence this type of task performance. The increase in avoidance during safe visits under small $\Delta \mathrm{F}$ conditions could be caused by the internal representation of the conditioned tone encompassing to a certain extent that of the safe tone. Since the effect is already observed in the group trained with the 0.75 octave $\Delta \mathrm{F}$, the width of this internal representation extends at least 0.75 octave below the conditioned sound. This is unusually wide given the critical bandwidth in the auditory cortex of C57BL/6 mouse (Ehret, 1976; King et al., 2015) but is comparable to the generalization width of latent-inhibition observed using the same Audiobox paradigm in a previous study (de Hoz and Nelken, 2014).

Mice showed better discrimination performance when the conditioned tone was of higher frequency than the safe tone. This asymmetry in behavior is unlikely the result of a differential innate/prior knowledge about the stimuli's frequency, since baseline activity was comparable across all tested frequencies. We hypothesize that the asymmetry was caused by an asymmetrical stimulus generalization, that the generalization around the conditioned tone was stronger towards the higher frequency. Similar asymmetrical generalization has been reported by Bang et al. (Bang et al., 2008) in rat using fear conditioning. They found that rats showed differential responses to the CS+ and CS- only 
when $19 \mathrm{kHz}$ pips, but not $4 \mathrm{kHz}$, were conditioned, although both sound stimuli were equally neutral before conditioning and elicited similar level of freezing behavior.

Overall the data support the hypothesis that perception of isolated stimuli (in a memory-based task) is constrained by a pre-wired circuitry underlying auditory processing. The uncommon wide and asymmetrical generalization, in line with our previous results (de Hoz and Nelken, 2014) and those of others (Ehret, 1976; Klink et al., 2010), suggests that pure tone frequency perception may be determined by the tonotopic organization of peripheral and subcortical areas, where neurons with wide and asymmetrical tuning curves have been recorded (Egorova et al., 2006; Taberner and Liberman, 2005). The fine acuity of behavioral discrimination reflected in low JNDs values can be achieved through the integration of information across wide tuning curves, as has been suggested for both the visual and the auditory system (Ehret and Merzenich, 1985; Kittel et al., 2002; Regan and Beverley, 1985). The steep slopes of tuning curves can also convey substantial information about frequency differences (Skottun et al., 2001; Taberner and Liberman, 2005). In either case, in order to extract discriminative information, the comparison between the responses elicited by different frequencies is necessary, and this comparison is not available in the same manner in a memory-based task where one of the frequencies needs to be pulled from memory. That these comparisons modulate behavior is reflected in the effect that previous history has on current performance (Chambers et al., 2017; Heffner and Masterton, 1980). Neurons with ultra-fine tuning have been described in the auditory cortex of humans (Bitterman et al., 2008). While these are not the norm, it is also not clear how their activity would help since a memory trace of previous activation would rarely overlap with the current response.

\subsubsection{Valence has a secondary modulation influence on generalization}

A stimulus of negative valence tends to have stronger behavioral and psychological impact on aspects such as speed of task learning (Chen and Bargh, 1999), generalization width (Dunsmoor and Paz, 2015; Schechtman et al., 2010) and perceptual learning (Åhs et al., 2013; Aizenberg and Neimark Geffen, 2013; Li et al., 2008; Resnik et al., 2011; Shalev et al., 2018). As a result, we would expect to observe wider generalization gradient towards the conditioned stimuli than the safe stimuli. Our findings are consistent with this view but only for $\Delta \mathrm{F}$ below 0.75 octave, suggesting that valence has a secondary modulation 
influence on generalization that becomes visible only as the discrimination becomes more difficult.

Responses towards the safe and conditioned tones varied differently with changes in $\Delta \mathrm{F}$. While for the conditioned tone decreases in $\Delta \mathrm{F}$ affected mainly the speed of avoidance learning but not the final level of avoidance, for the safe tone the same changes had a dramatic effect on the final level of nose-poking response. Also, smaller $\Delta \mathrm{F}$ had a more prominent narrowing effect on generalization gradients around the safe tone. Thus, positive and negative associations affect behavior differently. Physiological data suggest that negative and positive associations are processed in distinct but overlapping networks (Beyeler et al., 2016; Janak and Tye, 2015; Nieh et al., 2013). In mice, recent research on the sense of taste found that anatomically separated projections imposed different valence on sweet or bitter tastes (Wang et al., 2018).

Anxiety influences learning and generalization (Dibbets and Evers, 2017; Dunsmoor and Paz, 2015; Resnik and Paz, 2014). In the PPI data, acoustic startle responses were larger in mice trained in the Audiobox with a smaller $\Delta \mathrm{F}$ when the conditioned sound was used as the PPI background. Since greater startle reflex is associated with higher level of anxiety (Poli and Angrilli, 2015), the increased startle with smaller $\Delta \mathrm{F}$ could reflect the differential level of stress in the Audiobox between the groups trained with small $\Delta \mathrm{F}$ and those trained with larger $\Delta \mathrm{F}$. This might have caused the shift in the generalization gradient. However, no shift was observed in the Nlgn2 KO mice, a model of anxiety (Babaev et al., 2016; Parente et al., 2017). These mice showed decreased avoidance of the conditioned tone, a finding consistent with recent observations of learning impairments in mice that have an equivalent KO of the Nlgn2 protein (Chen et al., 2017; Jiang et al., 2018). However, and independent of possible learning impairments, mice lacking Nlg2 are highly anxious, making it safe to argue that overgeneralization does not always accompany increased anxiety. The asymmetrical generalization of animals trained with small $\Delta F$ s need not be the result from increased anxiety levels, a finding inconsistent with studies that have suggested that overgeneralization can underlie anxiety disorders (Laufer et al., 2016; Lissek et al., 2010). 


\subsubsection{Discrimination performance and generalization are differentially modulated by past experience}

In our paradigm, mice did not benefit from their past training experience when a new conditioned tone was introduced, independently of whether this increased or decreased the new safe-to-conditioned $\Delta \mathrm{F}$ distance. After re-training mice reached similar, sometimes even worse, performance than mice without prior training (e.g. animals trained first with $14 \mathrm{kHz}$ as conditioned tone and then with $9.8 \mathrm{kHz}$, versus mice trained directly with 9.8 $\mathrm{kHz}$ as conditioned tone). Human and animal studies on learning transfer under different stimulus classes suggest that the influences by initial training were based on implicitly learned integration of information about the stimuli, procedure, and cognitive skills (Kurt and Ehret, 2010; Liu et al., 2008; Ortiz and Wright, 2009; Spiering and Ashby, 2008). In our task, the different aspects of the experimental design (group housing for long period of time, ad libitum performance, absence of deprivation and automatic detection) meant that the task required very little procedural learning on the part of the mice. This may explain the small effect from past experience on subsequent training.

Generalization, however, was found to be affected by the summation of the animals' training history, consistent with findings obtained in pigeons on light wavelength generalization (Blough, 1969). However, it is interesting to notice that this summation effect did not depend on the chronological order of training, since we observed identical generalization gradients in mice trained with 9.8 and $14 \mathrm{kHz}$, independently of which tone was conditioned first.

\subsubsection{The dissociation between perceptual discrimination and generalization}

Training in the Audiobox had an effect on discrimination acuity measured in a subsequent PPI test. In line with previous studies (Amitay et al., 2006; Resnik et al., 2011; Watanabe et al., 2001), we saw a moderate improvement in acuity around the safe tone. Aversive learning, however, only led to an improvement in acuity around the conditioned tone when the safe-to-conditioned $\Delta \mathrm{F}$ was large. Overall, the wider generalization of the large $\Delta \mathrm{F}$, but not the narrow generalization of small $\Delta \mathrm{F}$, was accompanied by increased acuity. Our results are not consistent with the view that it is a decrease in discrimination acuity caused by aversive learning that leads to over-generalization (Laufer and Paz, 2012; Resnik et al., 2011; Schechtman et al., 2010). For example, in a recent study in which 
discrimination acuity was measured in the same way, wider generalization was accompanied by decreased acuity, whereas a training protocol that induced narrow generalization led to increased acuity following aversive learning (Aizenberg and Neimark Geffen, 2013). It is possible that the decreased acuity observed in other studies following aversive learning is a result of other factors, such as the use of different training paradigm (fear conditioning vs. avoidance learning) that might invoke different brain mechanisms. Fear conditioning may rely on a fast, nonconscious-operated, amygdala-based, system (Kim and Jung, 2006; LeDoux, 2000, 2014), whereas avoidance learning may rely on a more complex, consciousness-involved, cortical system (Barberini et al., 2012). Indeed, the physiological changes induced by classical or instrumental conditioning vary, which leads to the argument that plasticity also depends critically on the task characteristics (Irvine, 2018a).

The modulation in acuity is probably very local and quite dynamic as suggested by the fact that retraining to a new conditioned tone led to a reversal of the observed changed in acuity. Physiological plastic changes associated with behavioral learning have also been suggested to be very dynamic (Reed et al., 2011). The change in discrimination acuity might be epiphenomenal and not serve as the substrate of improved performance or learning itself (Irvine, 2018b; Sagi, 2011; Watanabe and Sasaki, 2015).

In conclusion, decisions based on absolute judgements, i.e. in the absence of a recently perceived comparative stimulus, are constrained by the physical distance between the tobe-discriminated stimuli. This suggests that wider representation filters are in place during absolute judgment decisions. As the $\Delta \mathrm{F}$ between the trained stimuli diminishes, safe and conditioned tones activate progressively more overlapping neuronal populations. In a relative judgement task, the recent activation of one neuronal population (responding to a safe sound, for example) might help detecting the differences in firing pattern of the currently activated neuronal population (responding to a conditioned sound, for example). Mechanisms such as adaptation of the recently activated neurons would decrease the overlap in the firing of the two populations and enhance the difference. In an absolute judgement task, however, the discrimination must rely on the memory of the representation of the safe and conditioned sounds. Based on our data, we argue that the memory traces for $\Delta \mathrm{F}$ of 0.5 octave or less overlap sufficiently for discrimination to become difficult. In this situation the animal becomes more conservative in its decision and its behavioral responses are more cautious. That discrimination is substantially impaired already at $\Delta \mathrm{F}$ of 0.5 
octaves suggest a strong role of pre-wired tonotopic organization and the involvement of subcortical areas, with wider tuning curves. Nevertheless, generalization of training to other similar stimuli depends on an interaction between the conditions of the training $(\Delta F)$, stimulus valence and past experience. This suggests that to perceptually distinguish stimuli (discrimination) and to respond to similar stimuli based on prior knowledge (generalization) are two processes that may rely on somewhat distinct circuits. 


\section{Chapter 2 Bidimensional generalization reveals hierarchical organization of acoustic dimensional processing}

\subsection{Introduction}

Sensory systems are sensitive to changes in the surrounding environment. The changes that organisms encounter during their daily life are essentially multidimensional. Indeed, a scene contains information that is not only blended across different modalities (Ahrens et al., 2015; Aluisi et al., 2018; Brosch et al., 2015), but also across different features of the same modality. Auditory stimuli, for example, can vary in duration or the way their frequency is modulated in amplitude or direction, among other things. A key question in the study of perception is how animals detect and use features from this multidimensional environment to make predictions. One way to investigate how animals perceive sensory stimuli and extract their features is through sensory discrimination tasks in which animals associate individual stimuli with behavioral outcomes (Kurt and Ehret, 2010; Ohl et al., 2001; Schulze and Scheich, 1999; Schulze et al., 2002; Wetzel et al., 1998).

The ability of animals to discriminate auditory stimuli varying along a single dimension has been extensively studied in many species, including rodents. Mice and rats have been trained to discriminate changes in sound frequency (Ehret, 1975; Fay, 1974; Kurt and Ehret, 2010; Rohrbaugh et al., 1971; Syka et al., 1996) or intensity (Kobrina et al., 2018; Rohrbaugh et al., 1971; Syka et al., 1996; Terman, 1970). Besides, discrimination can be done on the basis of modulation in frequency or amplitude. Frequency modulation refers to changes in the direction of the frequency change, i.e. upwards or downwards sweeps (Mercado et al., 2005; Ohl et al., 2001; Rybalko et al., 2006; Wetzel et al., 1998). Amplitude modulation refers to changes in the rate of the amplitude modulation (Kurt and Ehret, 2010; Schulze and Scheich, 1999). Despite abundant knowledge about the unidimensional discrimination, our understanding on multidimensional perception in rodents is still limited.

Rats are able to selective attend to one dimension of a stimulus (Mercado et al., 2005; Polley, 2006). Nevertheless, stimulus discrimination along single dimension could be influenced by stimulus features outside that dimensions. For example, intensity discrimination varies in acuity depending on the frequency of the sounds used (Florentine, 
1983; Heffner and Masterton, 1980). Similarly, duration (Nabelek and Hirsh, 1969; Schouten, 1985) and modulation rate (Gaese et al., 2006) affect direction discrimination of frequency modulation sweeps. These results suggest that while each dimension can be selectively perceived it can also interact with others.

Little is known about the way these dimensions are processed during auditory perception and the extent to which they are treated independently when identifying a sound. In the visual modality, where perception has been most intensively studied (Shibata et al., 2014; Watanabe and Sasaki, 2015), different stimulus dimensions (e.g. shape or orientation) are processed at different speeds (Ahissar and Hochstein, 1997). This finding led to the reverse hierarchy theory, which postulates that this differential response latency is caused by different stimulus dimensions being processed at different stations in the visual system. In the auditory modality, for example, this has not been studied as thoroughly but it is known that discriminations along the frequency dimension are easier than those along the loudness dimension (Polley, 2006) or the modulation rate of amplitude modulation (Kurt and Ehret, 2010).

In this series of behavioral experiments we used generalization, the ability to bundle different stimuli together based on their similarity, as a means to infer interactions between dimensions, as well as the dimension-hierarchy underlying auditory perception in mice. We trained mice in an absolute discrimination task with sound stimuli that differed in two dimensions. We then changed both dimensions independently of each other and used generalization to measure how mice perceived these changes and, therefore, the underlying dimensions. The sounds we used were logarithmic non-periodic frequency modulation (FM) sweeps varying in the following dimensions: frequency range of the sweep, direction of modulation (upwards or downwards), velocity of modulation, and duration. In an additional experiment we used, instead of sweeps, a sinusoidal amplitude-modulated (AM) tone which could vary along two dimensions: the carrier frequency and the modulation rate.

\subsection{Results}

A total of 77 female C57BL/6JOlaHsd mice were trained in an absolute sound discrimination paradigm in the Audiobox (TSE, Germany; Figure 0-1A). The task resembled a Go-No-go paradigm. In the Audiobox, mice live for the duration of the experiment (several weeks) while performing the task ad libitum. We first studied the perception of FM sweeps that differed in two out of four dimensions (Figure 2-1A; 
frequency range, direction, velocity of change, and duration). We then extended our research to periodic sound envelopes that differed in frequency and the rate of periodicity.

A
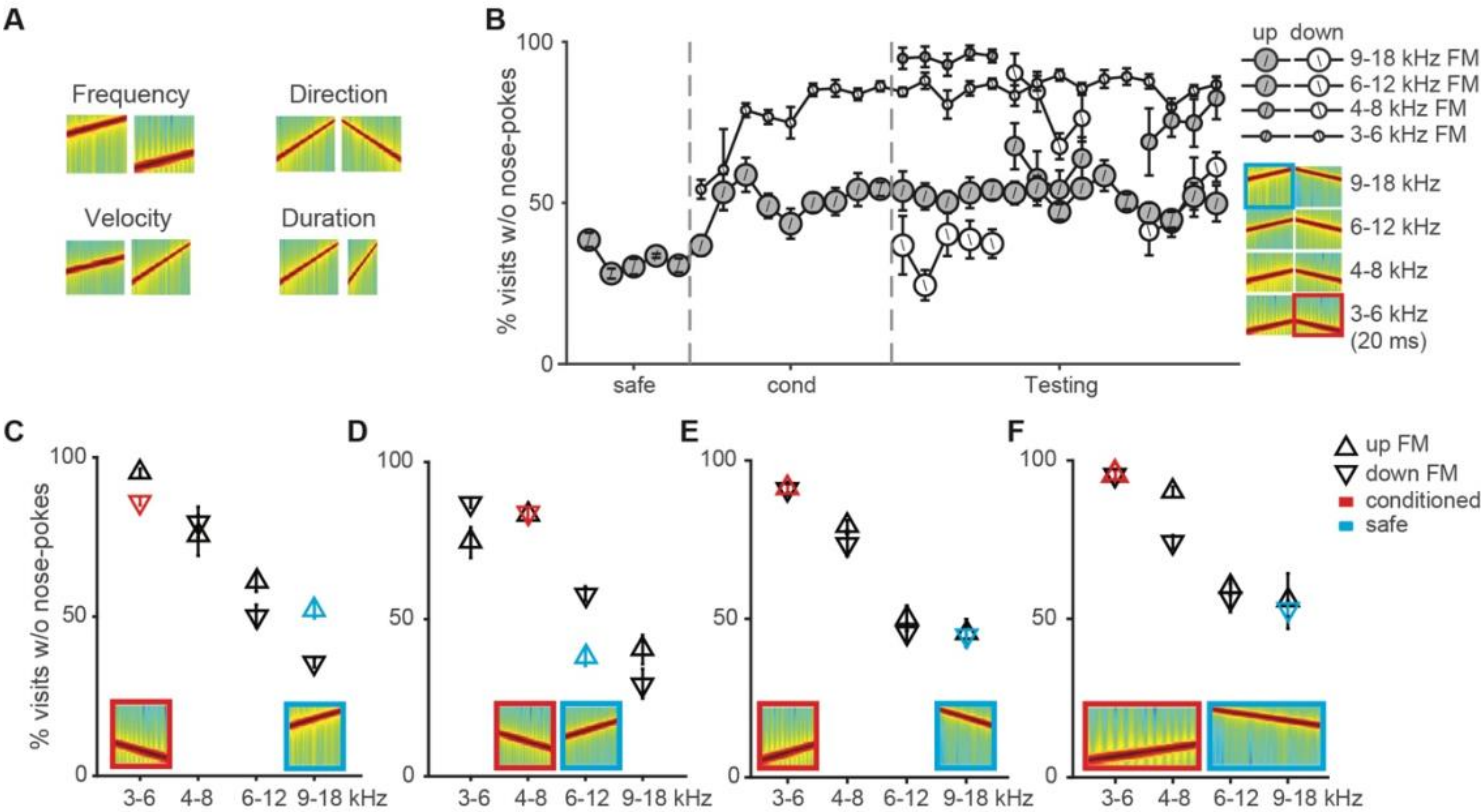

D
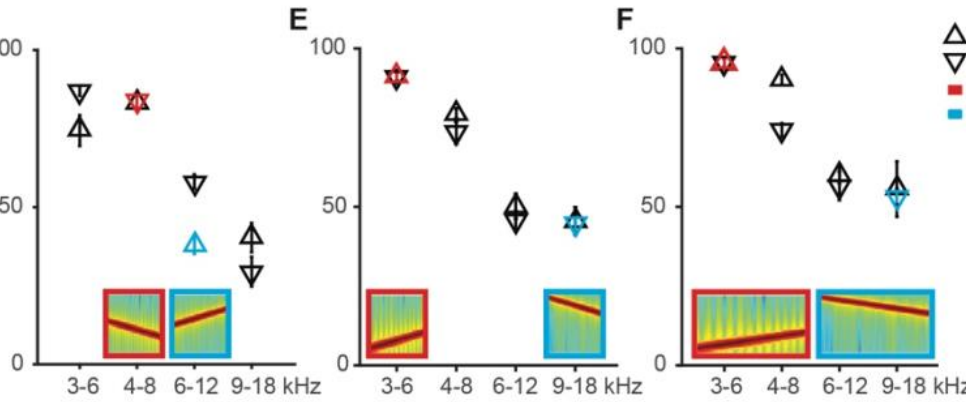

$\triangle$ up FM

$\nabla$ down FM

= conditioned - safe

$\hat{\psi}$

Figure 2-1 Generalization of FMs was along the frequency dimension, but not the direction

(A) 4 pairs of spectrograms of frequency-modulated sweeps differing in frequency (top-left), direction (top-right), velocity (bottom-left) or duration (bottom-right). (B) Performance during discrimination learning and generalization test of frequency-direction combinations. Mean daily performance expressed as the fraction of visits without nose-pokes for different type of visits. Spectrograms of tested stimuli are shown in bottom-right. The safe sound was 9-18 kHz upward FM pips (blue rectangle) and the conditioned sound was 6-3 kHz downward FM pips (red rectangle). (C) Average generalization gradients showing the mean response to each of the stimuli presented in (B). (D) Average generalization gradients for mice trained to discriminate FMs with partially overlapping frequency range. The safe sound was 6-12 kHz upward FM pips (blue rectangle) and the conditioned sound was $8-4 \mathrm{kHz}$ downward FM pips (red rectangle). (E) Average generalization gradients for mice trained to discriminate FMs with frequency-dependent preferred direction. The safe sound was $18-9 \mathrm{kHz}$ downward FM pips (blue rectangle) and the conditioned sound was 3-6 kHz upward FM pips (red rectangle). Sound duration used for (B-E) was $20 \mathrm{ms,} \mathrm{and}$ frequency modulation was at 50 octaves/s. (F) Average generalization gradients for mice trained to discriminate FMs with duration of $40 \mathrm{~ms}$ and frequency modulation at 25 octave/s. The safe sound was 9-18 kHz upward FM pips (blue rectangle) and the conditioned sound was 6-3 kHz downward FM pips (red rectangle). 


\subsubsection{Mice generalized mainly along the dimension of frequency range, but not FM direction.}

Mice were trained to discriminate FMs that differed in two dimensions, frequency range and FM direction (9 to $18 \mathrm{kHz}$ upward vs. 6 to $3 \mathrm{kHz}$ downward FMs). As reviewed in the introduction, the dimensions were chosen because they are known to be perceivable by rodents (Hage et al., 2003; Mercado et al., 2005; Wetzel et al., 1998; Zhang et al., 2003). Based on the ABR measurement in C57BL/6j mice, where lower thresholds were found for frequencies between 4 and $16 \mathrm{kHz}$ (Zhang et al., 2013), as well as the finding described in chapter 1 that a frequency distance of at least 0.75 octave elicits good discrimination, we mainly tested frequency ranges between 3 and $18 \mathrm{kHz}$. The choice of velocity of modulation was based on both physiology (Zhang et al., 2003) and behavioural (Gaese et al., 2006) data which showed high discriminability in rodents when using a velocity of 50 octave/s. Well trained mice, that showed stable discrimination performance (6 out of 9), were then tested with novel sounds varying along both dimensions (Figure 2-1B). Mice tended to either avoid or approach the novel sounds, reflecting whether they perceived them as similar to the 'conditioned' or the 'safe' sound respectively. Average generalization gradient is shown in Fig. 1C. We found that generalization occurred mainly across the dimension of frequency range, but not FM direction. Regardless of the direction, mice approached novel sounds of high frequency range $(6-12 \mathrm{kHz}$ and 9-18 kHz), and avoided novel sounds of low frequency range (3-6 kHz and 4-8 kHz). A two-way ANOVA revealed a significant main effect of frequency range $(F(3,40)=74.67, p=0)$, an effect of sweep direction $(F(1,40)=10.36, p=0.003)$ and a significant frequency $\times$ direction interaction $(F(3,40)=3.15, p=0.035)$. Indeed, a direct comparison between upward and downward sweeps (Figure 2-1C) revealed that, although frequency range was clearly the main determinant of behavior, for low frequency ranges, downward sweep elicited less avoidance than upward sweep of the same frequency range. One possible explanation for the minimal effect of direction is that the frequency at the beginning of the sweep plays a more decisive role. Thus, for a given frequency range, a downward FM starts with a higher frequency than the upward sweep and can therefore be perceived as more dangerous. This explanation would be in line with the dominant role of frequency in the discrimination.

It is possible that the choice of frequency over direction resulted from a more salient difference along the frequency dimension than the direction dimension, since the steps we used were not necessary comparable across dimensions. To test this possibility we narrowed the frequency difference and trained mice to discriminate sweeps of partially 
overlapping frequency range and opposite direction (6 to $12 \mathrm{kHz}$ upward vs. 8 to $4 \mathrm{kHz}$ downward FMs; $n=7$ ). Average generalization gradients revealed a similar pattern as before: animals discriminated the two sounds well and generalized mainly along the dimension of frequency range (Figure 2-1D). A two-way ANOVA revealed a significant main effect of frequency range $(F(3,48)=96.77, p=0)$ and a significant frequency $\mathrm{x}$ direction interaction $(F(3,48)=4.75, p=0.006))$, but no main effect of sweep direction $(F(1,48)=3.34, p=0.074)$.

Physiological studies have found a systematic representation of preferred sweep direction along the tonotopic axis at both the cortical and subcortical level, such that lowfrequency neurons prefer upward sweeps and high-frequency neurons prefer downward sweeps (Hage et al., 2003; Heil et al., 1992; Zhang et al., 2003). To further confirm that the frequency range dominance was not caused by the particular choice of frequency-direction combination, we tested animals trained with sweeps of frequency ranges that were modulated in the preferred direction (9 to $18 \mathrm{kHz}$ downward vs. 6 to $3 \mathrm{kHz}$ upward FMs, N =9). Our results showed that mice generalized again only along the dimension of frequency range (Figure 2-1E). A two-way ANOVA for generalization gradients revealed a significant main effect of frequency range $(F(3,64)=61.33, p=0)$, but no main effect of sweep direction $(F(1,64)=3.82, p=0.06)$ or frequency $\mathrm{x}$ direction interaction $(F(3,64)=$ $0.46, p=0.71)$.

Previous studies have shown that FM direction discrimination was limited by the sweep duration (Gaese et al., 2006; Nabelek and Hirsh, 1969; Schouten, 1985). Up to this point we had used sweeps of $20 \mathrm{~ms}$ duration based on discriminable durations in mice (Ehret, 1992). Since we use $20 \mathrm{~ms}$ sound stimuli in the above experiments, we now trained and tested mice with sweep duration at $40 \mathrm{~ms}$ ( 9 to $18 \mathrm{kHz}$ downward vs. 6 to $3 \mathrm{kHz}$ upward FMs; $n=7)$. Our results demonstrated that an increase in sweep duration did not change the generalization pattern (Figure 2-1F). The two-way ANOVA revealed a significant main effect of frequency range $(F(3,56)=51.48, p=0)$, but no main effect of sweep direction $(F(1,56)=3.65, p=0.06)$ or frequency $\mathrm{x}$ direction interaction $(F(3,56)=2.06, p=0.12)$.

Taken together, these results suggest that the dimension of frequency is perceived as more relevant and dominant than the dimension of sweep direction. It confirms the hypothesis that hierarchy also exist in the processing of low-level multidimensional information. And the perception of the frequency range of FMs is in a higher hierarchy than the perception of the sweep direction. 


\subsubsection{Hierarchical organization of FMs perception in mice}

To investigate the hierarchical organization of FMs perception, we then tested another three groups of mice, again using sounds that combined two of four dimensions (frequency range, direction, velocity of modulation, and duration). As mentioned before, we chose specific values based on behavioral studies on single dimension discrimination, as well as the properties of neurons in the auditory system. For the velocity of FMs, we chose 50 and 100 octave/s, which are velocities within the range (25-250 octaves/s) of velocities that elicit good responses in neurons (Hage and Ehret, 2003). For the duration of FMs, we used 20 and $80 \mathrm{~ms}$, since mice had a categorical short-long boundary at around $25 \mathrm{~ms}$ (Ehret, 1992)

First, we trained mice to discriminate sounds that differed in sweep velocity and direction but had the same center frequency of $8 \mathrm{kHz}$ (100 octave/sec upward FM pips as the safe sound vs. 50 octave/sec downward FM pips as the conditioned sound; $n=8$ ). Average generalization gradients showed that mice made their decision mainly based on the sweep direction, but not velocity (Figure 2-2A). A two-way ANOVA analysis revealed a significant main effect of sweep direction $(F(1,56=116.21, p=0)$, a significant main effect of sweep velocity $(F(3,56)=4.67, p=0.006)$, but no interaction $(F(3,56)=1.27, p=$ 0.30). Overall, the effect of sweep velocity on animals' performance was less pronounced than the effect of sweep direction, which suggests that sweep direction is higher in the hierarchy than sweep velocity. This result replicated our finding from before that the perception of sound dimensions was hierarchically organized. 
A

B
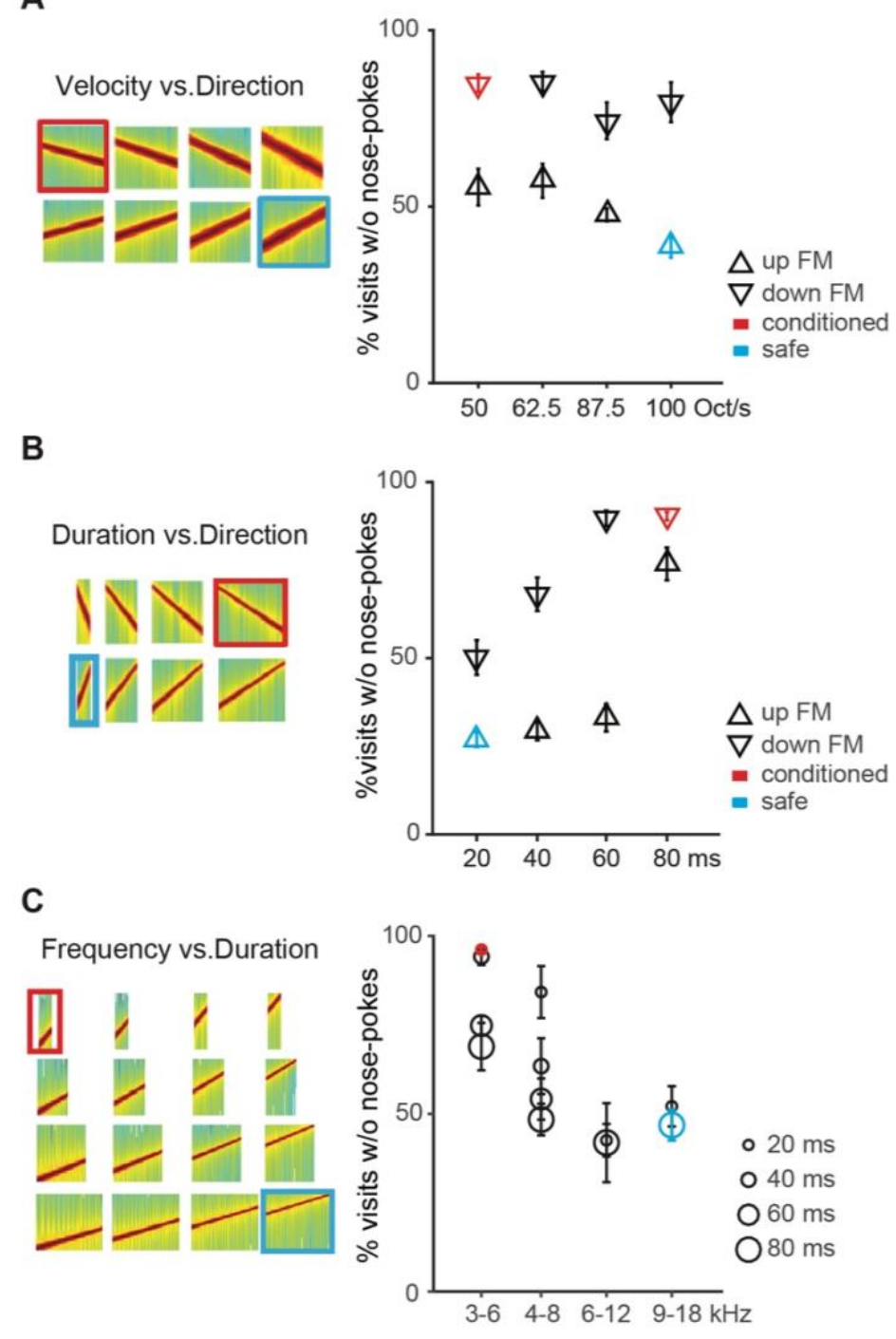

Figure 2-2 Multidimensional generalization of FMs

(A-left) Spectrograms of FMs used in the generalization test for the combination of velocity and direction dimensions. The safe (blue) and conditioned (red) sounds were marked. (A-right) Average generalization gradient for the dimension combination of sweep velocity and direction revealed that generalization was mainly along the direction dimension. (B) Same as (A) for the combination of sweep duration and direction dimensions. Average generalization gradients (right) revealed an effect on behavior of both dimensions. (C) Same as $(\mathbf{A}, \mathbf{B})$ for the combination of frequency range and duration dimensions. Average generalization gradients (right) showed that both dimensions controlled animals' behavior.

For mice tested with the combined dimensions of duration and sweep direction (Figure 2-2B; $20 \mathrm{~ms} 4-16 \mathrm{kHz}$ upward FM pips as safe vs. $80 \mathrm{~ms}$ 16-4 kHz downward FM pips as conditioned; $n=10$ ), response changed monotonically as a function of changes in both sound dimensions. It is worth noting that changing the duration of the FM of fixed frequency range also alters the sweep velocity. Here, the sweep velocities for the safe and the conditioned sound were 100 and 25 Oct/s respectively, which were more different than 
before. Since we observed much weaker effect of sweep velocity on generalization before, we can therefore rule out the possibility that mice used the velocity dimension instead of the duration dimension for making decision in the duration-direction task. A two-way ANOVA revealed a significant main effect of sound duration $(F(3,72)=43.93, p<0.0001)$, a significant main effect of sweep direction $(F(1,72)=120.26, p<0.0001)$, and a significant duration $\mathrm{x}$ direction interaction $(F(3,72)=9.04, p<0.0001)$. This result indicates that mice could generalize along both dimensions simultaneously and the dimensions of sweep duration and direction may be on the same hierarchical level of auditory perception.

Finally, we investigated how mice generalized along the combined dimensions of frequency range and sound duration ( 9 to $18 \mathrm{kHz} 80 \mathrm{~ms}$ FM pips as safe vs. 3 to $6 \mathrm{kHz} 20$ ms FM pips as conditioned; $n=8$ ). We found that both dimensions exerted strong control over the animals' behavior (Figure 2-2C). A two-way ANOVA revealed a significant main effect of frequency range $(F(1,84)=25.08, p=0)$, a significant main effect of duration $(F(1,84)=16.98, p=0.0001)$ and a significant interaction between the two dimensions $(F(5,84)=2.86, p=0.02)$.

Taken together, the results indicate that the sound dimensions can be perceived independent from each other. In addition, and following the postulate of the reverse hierarchy theory that information processing along the sensory pathways is hierarchically organized and high-level information is accessed first (Ahissar et al., 2009), we could infer the hierarchical organization of FMs perception in which the dimension of frequency is high up, followed by sound duration and sweep direction, and the dimension of sweep velocity is down in the hierarchy.

\subsubsection{Mice had the flexibility to be trained to do unidimensional categorization.}

We next examined whether the mice that showed generalization gradients along both tested dimensions can be trained to ignore one of the dimensions. Here, for mice trained with the combined dimensions of direction and duration, we subsequently trained half of them with an additional conditioned sound (the 2 nd conditioned tone) that was the same in direction as the initial conditioned tone but differed from that in duration (Figure 2-3A right, $n=5$ ). Thus the safe and the conditioned tones differed only in the direction dimension. After the 2 nd conditioning, the added conditioned tone, which had been treated 
like the safe tone, was now avoided by the mice (Figure $2-3 \mathrm{~A} ; 88 \%$ of avoidance, $\mathrm{d}^{\prime}=$ 1.45). Learning not only affected the new conditioned sound, but also novel sounds in between the safe and conditioned ones (Figure 2-3A). The generalization gradients was now mainly along the direction dimension. A two-way ANOVA for performance of the retrained mice revealed a significant main effect of sweep direction $(F(1,32)=283.03, p=$ $0)$, but no effect of duration $(F(3,32)=2.43, p=0.08)$ or interaction between those two dimensions $(F(3,32)=2.63, p=0.07)$.
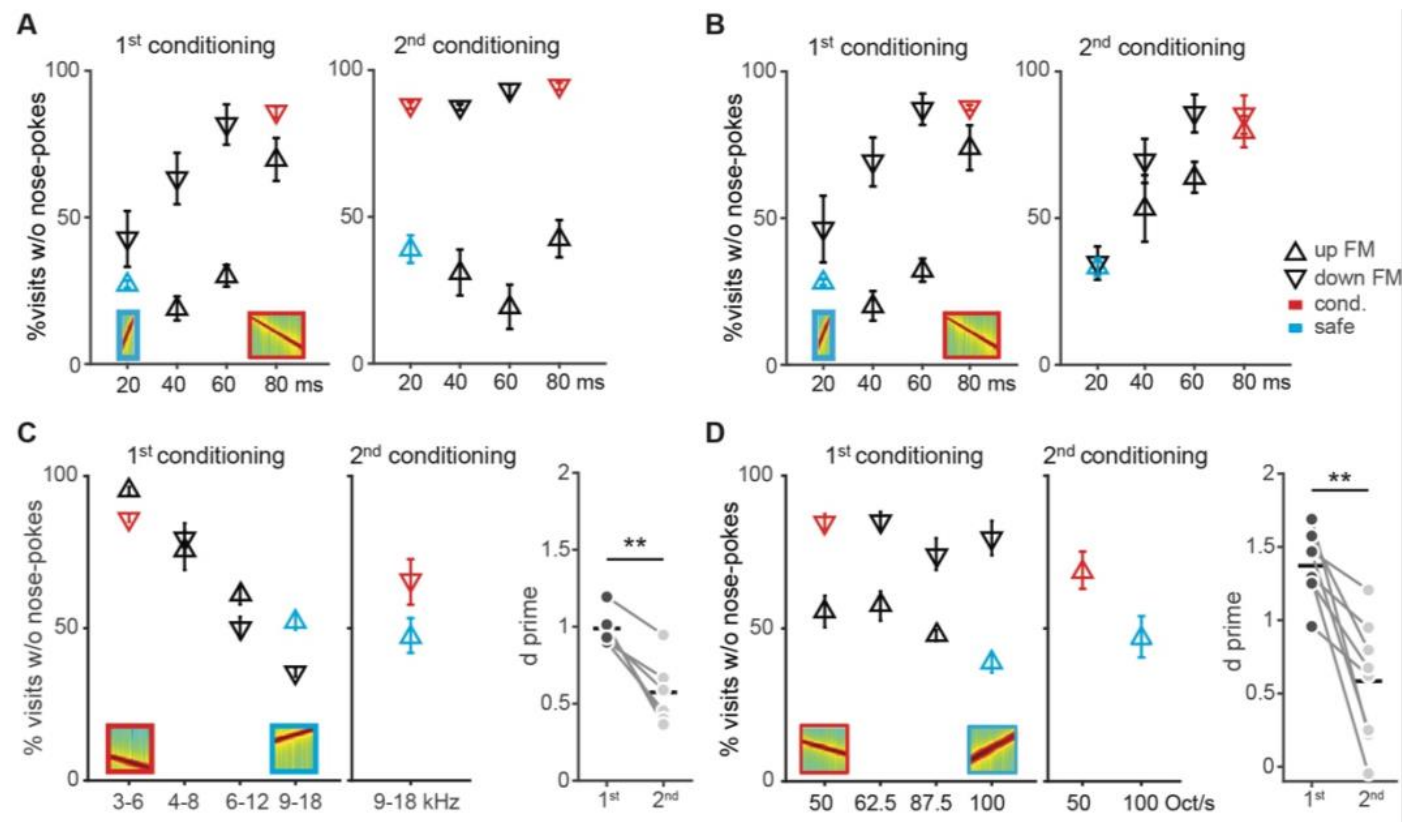

Figure 2-3 Mice can be trained to do unidimensional categorization.

(A-B) Average generalization gradients after the 1 st conditioning (left) and the 2 nd conditioning (right). (A) Mice initially generalized along both direction and duration dimensions (left), and after training, shifted generalization to be only along the direction dimension (right). (B) Same as (A) for mice initially trained with combination of direction and duration (left) and subsequently trained to do duration discrimination (right). (C) Average generalization gradients for mice initially trained with the combination of frequency and direction dimensions (left). Discrimination performance for mice after 2 nd training to discriminate FMs direction (middle). d' after the 1 st and 2 nd conditioning for individual mice. (D) Same as (C) for mice initially trained with the combination of sweep direction and velocity dimensions and subsequently trained to do velocity discrimination.

Similarly, for the other half of the mice that were initially trained with the combined dimensions of direction and duration, we added an additional conditioned tone to make the safe and the conditioned tones differed only in the duration dimensions (Figure 2-3B). Interesting, the avoidance to the new conditioned tone was not largely changed since it had been avoided after the first conditioning (from $74 \%$ to $79 \%$; d'2nd $=1.24$ ), but animals' responses to novel sounds in between the safe and conditioned ones changed. Mice now 
mainly generalized along the duration dimension (Figure 2-3B right). A two-way ANOVA revealed a significant main effect of sweep duration $(F(3,24)=15.73, p=0)$, a weaker but significant main effect of direction $(F(1,24)=4.41, p=0.046)$, but no interaction between those two dimensions $(F(3,24)=0.77, p=0.524)$. Thus, changes in the task conditions can shift the behavior from one where both dimensions are used (bidimensional generalization) to one in which one dimension is ignored (unidimensional categorization).

We further tested if it is possible to train mice that initially generalized mainly along one of the two tested dimensions to sort stimuli based on their non-preferred dimension. , Animals trained with frequency range and direction, where the low frequency downward pip was the conditioned sound and the high frequency upward pip was the safe sound, were now trained with a high frequency downward sound as conditioned (Figure 2-3C middle). Unlike what we observed before that regardless of their previous response to the new conditioned sound mice learnt to avoid it and reached good discrimination (d' above 1), here, the 2 nd conditioning led to an increase in avoidance to the new conditioned sound (from $35 \%$ to $65 \%$ ) but their discrimination was not as good as the 1 st conditioning (Figure 2-3C right; paired t-test for d', $p=0.002$ ). This is consistent with the finding in the visual modality that the processing of lower-level information is slower and more difficult (Ahissar and Hochstein, 1997). Similar result was observed in mice previously trained with the combined dimensions of direction and velocity (Figure 2-3D). Retraining the mice to do velocity discrimination increased avoidance to the new conditioned sound (from $56 \%$ to $69 \%$ ) but weaker performance (Figure 2-3D right; paired t-test for d', $p=0.003$ ).

In summary, mice could be flexibly trained to use single dimension as the behavioral relevant ones, however, the performance was weaker for the non-preferred dimensions.

\subsubsection{When the discrimination task was along the non-preferred dimension, learning becomes more localized.}

To further characterize the interaction between dimensions of different hierarchical level, we examined the effect of training naïve mice with the non-preferred dimension on their responses to stimuli varying along both preferred and non-preferred dimensions. In particular, we accessed whether mice can generalize only along the trained dimension (non-preferred) and ignore the other one (preferred). It is believe that task using the nonpreferred dimension recruits lower level representations, which have the same specificity 
character as the lower level populations that underlie it (Ahissar et al., 2009). Direction selectivity in the auditory system has been found to be tonotopically organized with neurons of low characteristic frequency being sensitive to upward sweeps (Heil et al., 1992; Kuo and Wu, 2012; Zhang et al., 2003). We expected, therefore, direction discrimination would be frequency specific.

Mice were trained to discriminate FM pairs of opposite direction but the same frequency range (Figure 2-4A, 5-10 kHz upward FM pips as safe vs. 10- $5 \mathrm{kHz}$ downward FM pips as conditioned, $n=8$ ). Generalization was later measured with FMs of different frequency range and different direction $(8-4 \mathrm{kHz}$ downward FM pips and 6-12 kHz upward FM pips). As we expected, learning did not generalize across the frequency range as revealed by strong avoidance to both tested sounds (Figure 2-4B). Subsequent conditioning to a FM of lower frequency range (8-4 kHz downward FM pips) led to direction discrimination across wider frequency range (Figure 2-4C). But learning was still limited to the trained frequency range, as reflected by no categorical responses to the FMs of frequency range beyond the trained one (Fig. 4B; 3-6 kHz upward and $18-9 \mathrm{kHz}$ downward FM pips). Therefore, learning along the non-preferred dimension was more specific, suggesting fine neural representation where learning occurred.

A

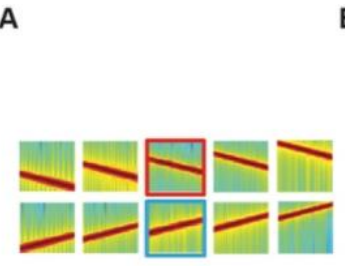

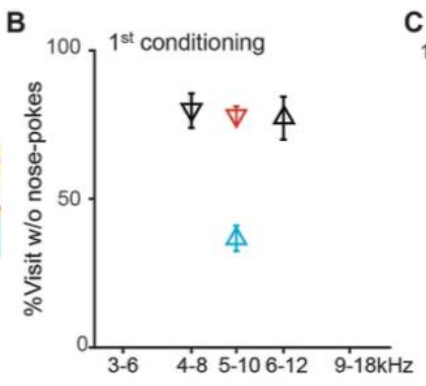

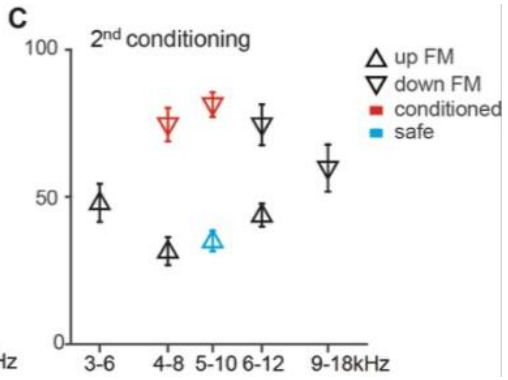

Figure 2-4 Generalization along the non-preferred dimension was localized

(A) Spectrograms of FMs used in the test. The safe (blue) and conditioned (red) sound used in the $1_{\text {st }}$ conditioning were marked. (B) Average generalization gradients after the 1 st conditioning with FMs differed in direction. (C) Average generalization gradients after the 2 nd conditioning. (A-C) The safe (blue) and conditioned (red) sound were marked.

\subsubsection{Hierarchical organization of 'envelope' perception in mice}

The 'fine-structure' and 'envelope' are two important components of acoustic stimuli. In the experiments in which we studied the hierarchical organization of FMs perception, we mainly focused on the 'fine-structure' dimensions, which were determined by the fast pressure variations. In the following experiments we investigated how the 'envelope', 
which is the contour of this fine-structure, was perceived. Since amplitude modulations and periodic envelope are important characteristic for acoustic communication signals (Leaver and Rauschecker, 2010), here, we trained and tested mice with 100\% sinusoidal AM stimulus (differing in dimensions of carrier frequency and modulation rate, Figure 2-5A left) or with periodic envelopes (differing in dimensions of carrier frequency and repetition rate, Figure 2-5B left). The carrier frequencies differed in 1 octave, based on results described in chapter 1. We mainly focused on AMs with low modulation rate (below $20 \mathrm{~Hz}$ ), as it has been found in gerbils that AM encoding is temporal (synchrony code) for low modulation rate but spatial (rate-place code), for high modulation rate (Schulze and Scheich, 1999). We chose AMs of 5 or $20 \mathrm{kHz}$ ( $\Delta \mathrm{F}$ of 2 octaves) modulation rate, since behaviorally mice have been shown to discriminate AMs differing in 1 octave modulation rate (Kurt and Ehret, 2010). As neurons in AC selectively prefer stimulus rates ranging from 2 to $15 \mathrm{~Hz}$ (Kilgard and Merzenich, 1999), we trained mice with repetition rates between 2 and $8 \mathrm{~Hz}$ ( $\Delta \mathrm{F}$ of 2 octaves).

A
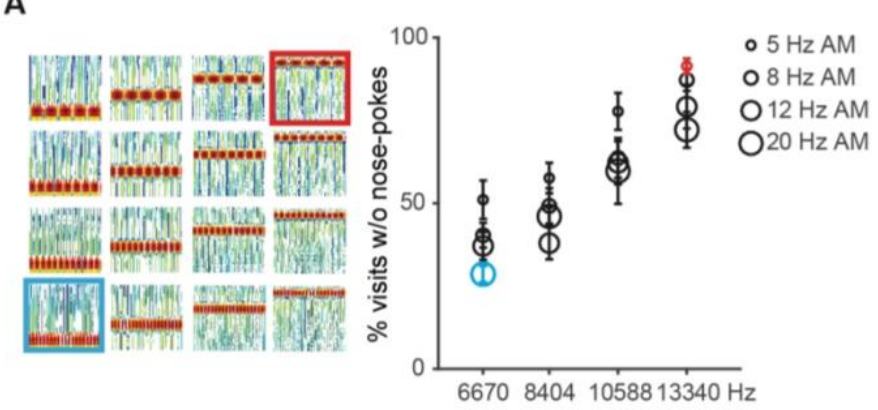

B

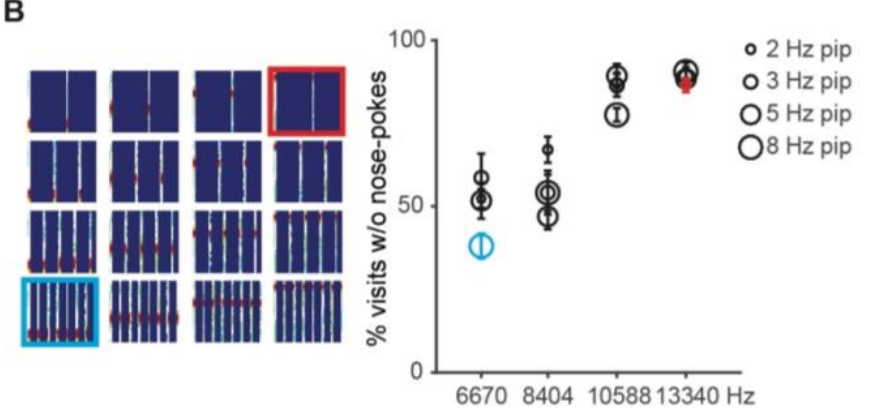

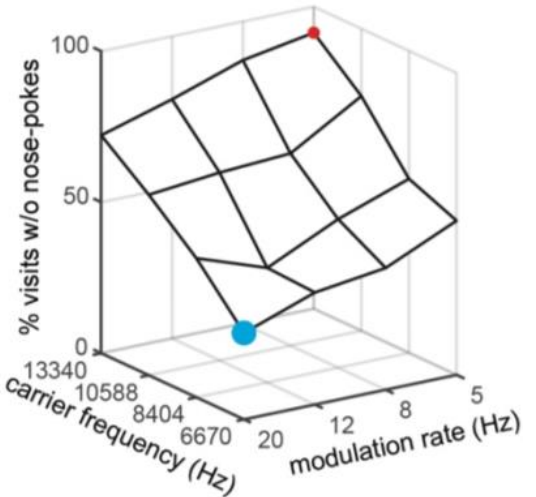

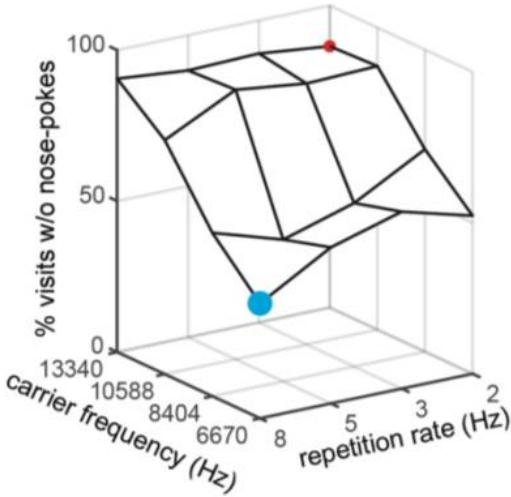

Figure 2-5 Bidirectional generalization of periodic sound envelopes

(A-left) Spectrograms of FMs used in the test. (A-middle and right) Average generalization gradients plotting the mean response proportion to each of the stimuli presented in the task for the combination of tone frequency and AMs rate dimensions. The same information is plotted as a circle plot (middle) and as a surface plot (right). (B) Same as (A) for the combination of ton 
frequency and tone-pip repetition rate dimensions. For each figure, the safe and conditioned sounds were marked in blue and red, respectively.

Figure 2-5A and B show how animals in each group responded to trained and novel sounds after training respectively. We found that in both groups performance was influenced by both tested dimensions, but was more strongly impacted by the dimension of carrier frequency. Alternation in carrier frequency elicited bigger changes in performance compared with changes the dimension of periodicity rate.

For mice trained with AM, a two-way ANOVA revealed a significant main effect of carrier frequency $(F(3,112)=53.15, p=0)$, a significant main effect of modulation rate $(F(3,112)=8.98, p=0)$, but no interaction between those two dimensions $(F(9,112)=0.47$, $p=0.89$ ). This suggests that the processing of the carrier frequency and the AM modulation rate may be of the same hierarchy but parallel processes. Indeed, discrimination of pure tone frequency, on the one hand, and of modulation rates of an AM pure tone, on the other, involves different neural and perceptual processes (Langner, 1992; Langner et al., 1997, 2002).

For mice retrained with periodic envelops, a two-way ANOVA revealed a significant main effect of carrier frequency $(F(3,80)=92.59, p=0)$, a significant main effect of repetition rate $(F(3,80)=3.18, p=0.028)$ and significant interaction between those two dimensions $(F(9,80)=2.38, p=0.019)$. This indicates that the processing of the carrier frequency and the repetition rate may be of the same hierarchy and with overlapped neural representation in the auditory system.

\subsubsection{The combination of sound dimensions used in the task influenced task performance}

In the visual modality, learning becomes more specific in difficult tasks in which lowerlevel processing is required (Ahissar and Hochstein, 1997). To assess whether this is also true for the relative low-level auditory dimensions, we compared learning for mice trained to discriminate stimuli based on single dimension or a combination of two dimensions. To compare learning, we calculated the average percentage of visits without nose-pokes in blocks of 4 visits starting after the first conditioned visit (Figure 2-6A). As described in chapter 1, we did this separately for conditioned visits (red), safe visits that followed a conditioned visit (blue), and safe visits that preceded one (gray). The division of safe visits intended to test the effect of a conditioned visit on the safe ones. 
Three groups of mice were compared for unidimensional discrimination learning. The tone discrimination data were from mice described in chapter 1 that were trained with $\Delta \mathrm{F}$ of 1 octave. For direction discrimination, mice described in Figure 2-4B were used. These are mice that were trained to discriminate between FM sounds of identical frequency range (5-10 kHz) but opposite direction of modulation. The third group was conformed of mice trained to do FM duration discrimination with 4-16 kHz upward FM pips and either $20 \mathrm{~ms}$ long in the case of the safe sound and $80 \mathrm{~ms}$ long in the case of the conditioned sound. We found that learning to discriminate tone frequency or duration was faster and easier than direction (Figure 2-6 B-C). A quantification of the $d$ ' values revealed significant differences between tone frequency or duration discrimination and direction discrimination (Figure 2-6E; unpaired t-test, pfreq-dir $<0.0001$, pdura-dir $=0.0002$ ), but no difference between the former frequency and duration discrimination (Figure 2-6E; unpaired t-test, pfreq-dura $=0.34)$. This finding matches the inference based on the bidimensional generalization we described before that the direction dimension is of lower hierarchy than the frequency and duration dimension. Therefore, learning becomes harder with lowerlevel dimensions. 

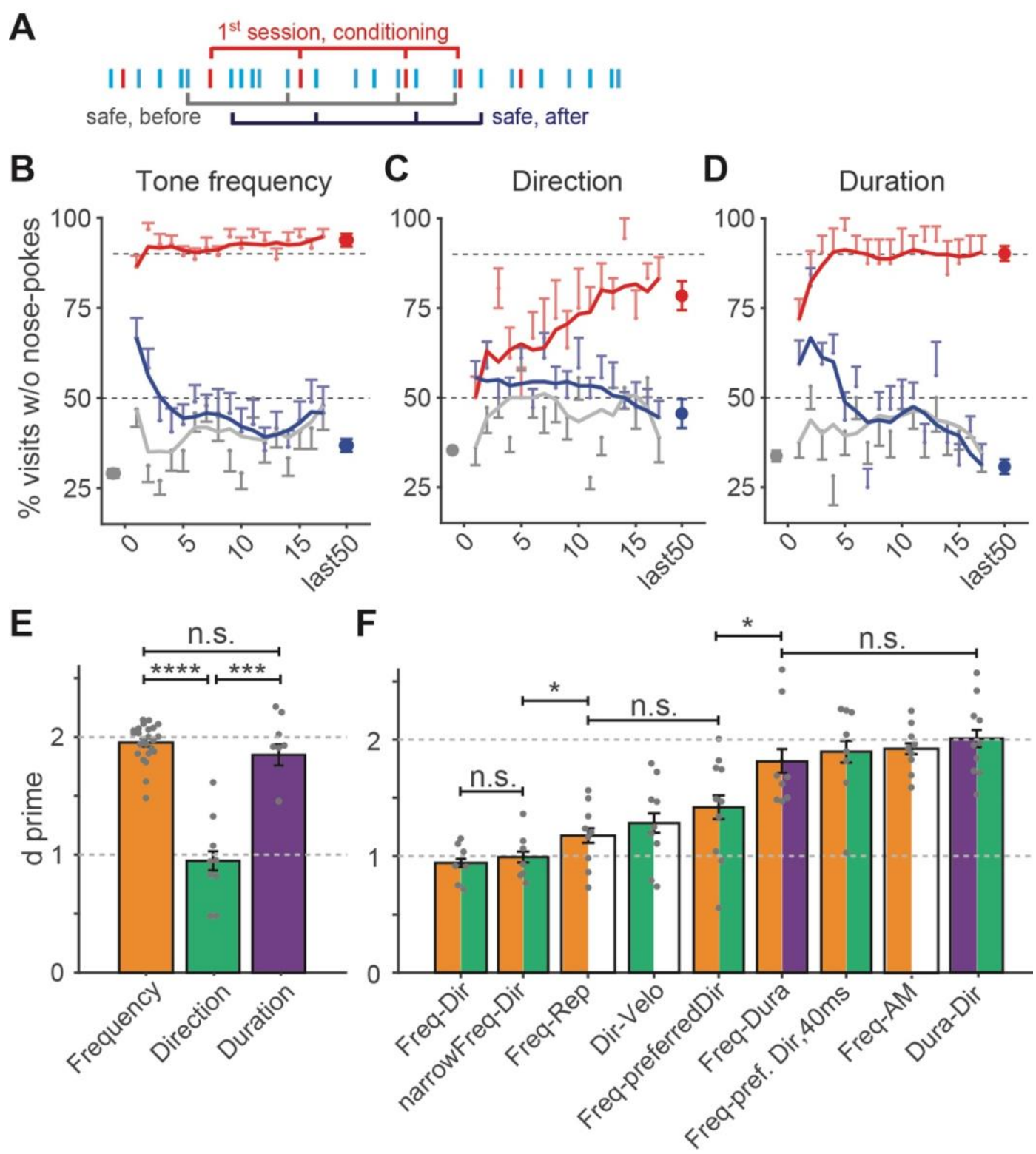

Figure 2-6 Learning curves and discrimination comparison

(A) Schema of learning curve analysis in blocks: average of blocks of 4 visits starting after the first conditioned visit (red) separately for safe visits (light blue) that follow conditioned visits (blue brackets), safe visits that precede conditioned visits (gray brackets), and conditioned visits (black brackets). (B-D) Performance analysis during single dimension discrimination learning. Baseline performance (black dot) calculated as the mean response over the last 50 safe visits before the first conditioned visit (safe-only phase). Mean performance in each session was plotted for safe visits following conditioned visits (dark blue), safe visits preceding conditioned visits (gray), and conditioned visits (red). Learning curves were smoothed using a 5 moving average filter. (E) d' values of individual mice trained to do unidimensional discrimination. (F) d' values of individual mice trained to discriminate acoustic stimuli differing in 2 dimensions. Performance is ordered by increasing d' value. Three group arise with comparable d' values ('n.s.'). The transition from one group to another are significantly different (denoted by asterisk).

Average d' values for all 9 combinations of dimensions are shown in Figure 2-6F. We found that discrimination level, the d', depended on the combination of dimensions used in 
the task. The combination of frequency and frequency-dependent non-preferred direction (Figure 2-6F left 1 and 2; Figure 2-7 A, B) elicited weaker discrimination performance. Their d' did not differ significantly (Kruskal-Wallis H-test analysis of variance, $p=0.64$ ). For Figure 2-6F from left to right, the next 3 combinations (Figure 2-7 C-E) and the last 4 combinations (Figure 2-7 F-I) did not differed significantly from each other (H-test, pmiddles $=0.30$, pright $4=0.44)$. The d' of the left combinations differed significantly from the d' of the middle combinations ( $\mathrm{H}$-test, $p=0.003$ ), as did the middle and right combinations $(\mathrm{H}-$ test, $p<0.0001)$.It is interesting to note that the dimension combinations that induced good performance (the right-most 4 in Figure 2-6F) were either with duration dimension or tested with relative long sound duration, thus indicating the important role of duration in sound perception. Indeed it had been found that in rats the discrimination of sweep direction was better for FMs of longer duration (Gaese et al., 2006). Increasing duration, though, did not override the dominance of frequency over direction discrimination during the generalization test in our hands, but it did speed up the initial discrimination between safe and conditioned tones that differed in both frequency and direction. 

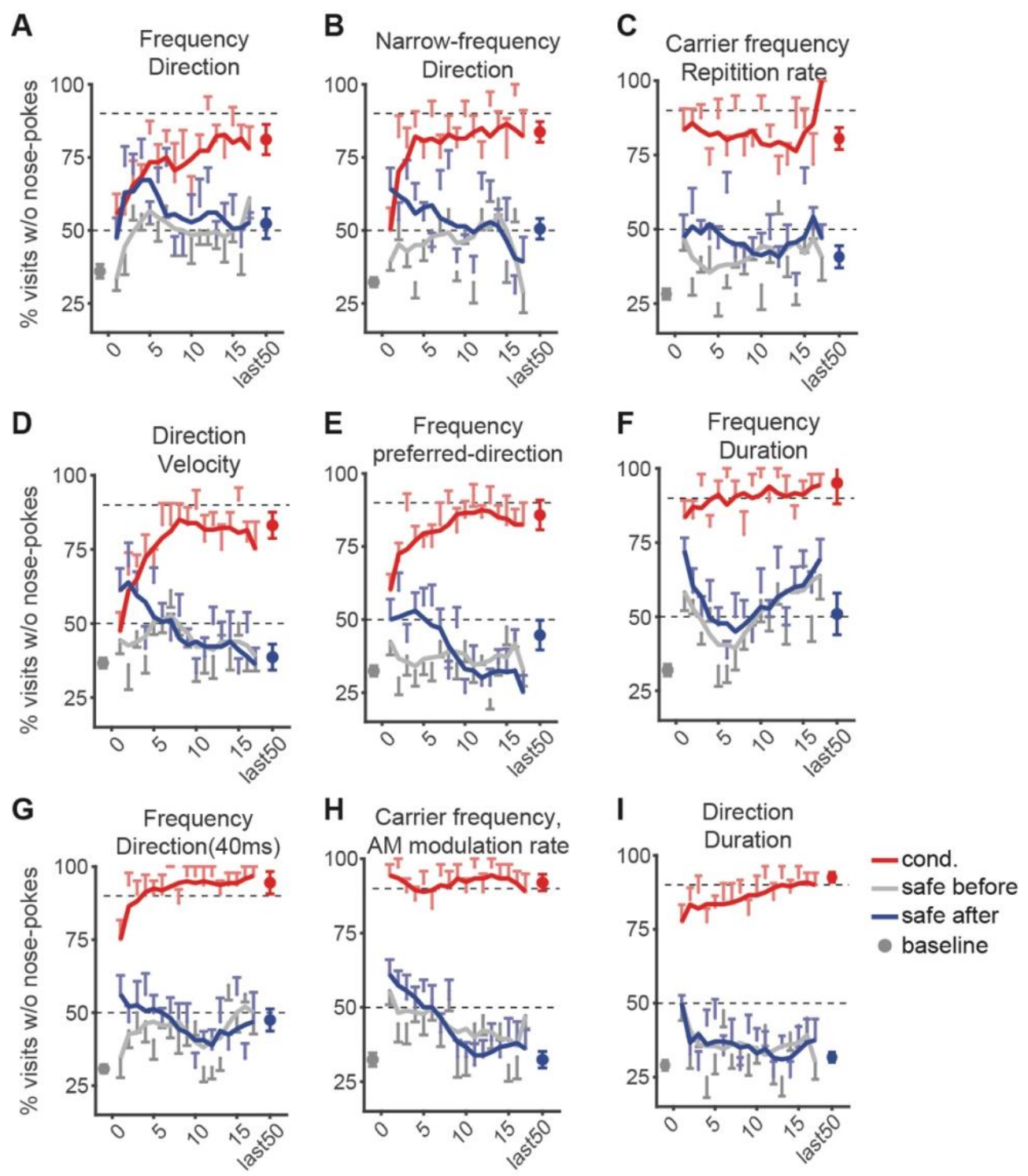

Figure 2-7 Learning curves for discrimination of stimuli that differed in $\mathbf{2}$ dimensions

(A-I) Performance during discriminate learning across acoustic stimuli that differed in 2 dimensions. Nine dimension combinations were tested separately in 9 groups of mice. Baseline performance (black dot) calculated as the mean response over the last 50 safe visits before the first conditioned visit (safe-only phase). Mean performance in each session was plotted for safe visits following conditioned visits (dark blue), safe visits preceding conditioned visits (gray), and conditioned visits (red). Learning curves were smoothed using a 5 moving average filter.

When envelope periodicity (Figure 2-7 C, and $\mathrm{H}$ ) was part of the sound, only when the periodicity was amplitude modulated did performance compare to that of mice trained with the dimension of tone frequency (Figure 2-6B). When the periodicity was in the repetition rate we saw deteriorated discrimination (Figure 2-6E, F; unpaired t-test, pfreq, freq-rep $<$ 0.0001, prreq, freq-AM $=0.454)$. Since we found an interaction between the frequency and the repetition rate, but not with $\mathrm{AM}$ rate, deteriorated performance may be a result of a distraction effect by the interaction between dimensions. 
Finally, we compared performance of mice trained with the dimension combination of frequency range and sweep direction but with different degree of dimension dissimilarity (Figure 2-7 A, B, E, G). As described in chapter 1, we found that the distance between stimuli influenced discrimination performance. But what is interesting is that although the direction dimension seemed to exert no effect on generalization gradients, changes in the direction dimension influenced learning. Pairing the frequency range with its preferred direction, which was found in physiological studies across species (Heil et al., 1992; Kuo and $\mathrm{Wu}, 2012$; Zhang et al., 2003), facilitated learning (Figure 2-6F). This indicates a correlation between behavior and physiology.

\subsection{Discussion}

Our study on multidimensional generalization in auditory modality in mice, revealed that the processing of acoustic dimensions is hierarchical organized and that whether acoustic dimensions are perceived independently of each other or combined depends on the two dimensions that are tested.

We show that mice could be trained to do absolute discrimination with complex tones, like FMs and AMs, differing along two acoustic dimensions. They were able to generalize learning to novel tones, reflected by either avoiding or approaching novel sounds similarly to the 'conditioned' or the 'safe' sound respectively. The generalization can happen along only one of the two tested dimensions, i.e. the frequency. Mice, however, had the ability to generalize to their non-preferred dimension, i.e. sweep direction, if they were actively trained to discriminate only along that dimension. Nevertheless, learning along the nonpreferred dimension was limited to a narrow range within the preferred dimension. Thus, our data support the hierarchical organization of acoustic feature perception. We found that when frequency range was one of the dimensions used, generalization occurred mainly across this dimension. Frequency may, thus, be high up in the hierarchy. More generally, our finding suggests that the perception of dimensions of the complex sound can be independent from each other. 


\subsubsection{Hierarchical organization of the low-level acoustic dimension processing}

Hierarchical processing of sensory information has been intensively studied in the visual modality (Grill-Spector and Malach, 2004; Hegdé, 2008; Hochstein and Ahissar, 2002; Markov and Kennedy, 2013). The influential reverse hierarchy theory posed by Ahissar and Hochstein (Ahissar and Hochstein, 1997) point out that along the cortical hierarchy, only the high-level abstract inputs are prioritized for perception. Later it has been suggested that this theory also underlies the auditory modality, comparing fine spectrotemporal acoustic features with abstract phonological representations (Ahissar et al., 2009; Nahum et al., 2008). Here, our data suggests that similar rule may also apply to the processing and perception of relative low-level dimensions, i.e. fine spectrotemporal acoustic features, in mice. For example, learning to discriminate along the frequency range led to faster learning than to discriminate along the sweep direction. For mice trained to discriminate stimuli that had dissimilarities on both dimensions, their decision was based only on the frequency range. Besides, the generalization of direction discrimination learning was limited to the frequency range of trained sounds. These results point to the same conclusion that frequency range was in a higher-level of the hierarchy than sweep direction.

Within the range of our tested dimensions, frequency seems to be high up the hierarchy, which is consistent with other studies in rodents. It been found in rats that discrimination along the frequency dimension is easier than those along the modulation rate of AM (Kurt and Ehret, 2010) and the loudness dimension (Polley, 2006). The study of wiggling call perception in untrained mice shows that both frequency and duration are critical to elicit maternal responsiveness (Ehret and Riecke, 2002; Geissler and Ehret, 2002), which matches our inference that frequency range and duration were at comparable levels of the hierarchy. Although among the acoustic features we tested, frequency is at the highest level of the hierarchy, it is possible that there are other higher-level sound features. Indeed, some studies in human report that the processing of sound texture, i.e. the collection of acoustic features, relies only on time-averaged statistics, but not on its fine temporal details (McDermott et al., 2013). 


\subsubsection{Neurophysiological consideration of dimension perception}

How is the hierarchical processing of acoustic feature reflected in the functional and anatomical organization of the auditory system? Tonotopy (spatial map of frequency) of auditory neuronal responses, which originates through mechanical properties of the basilar membrane in the cochlear, is preserved throughout the auditory pathway (Brugge, 1992). This may reflect a computational need to process different frequency components separately (Schreiner et al., 2000). However, to date no systematic sweep direction, velocity or duration gradients have been observed in AC or IC (Atencio and Schreiner, 2010; Hage and Ehret, 2003), although direction or velocity selectivity have been found in subgroups of neurons in AC and IC (Hage and Ehret, 2003; Heil et al., 1992). Direction selectivity, for example, has been found to be mapped differently depending on tonotopy (Heil et al., 1992; Kuo and Wu, 2012; Zhang et al., 2003). This is evident in both AC and IC where neurons responding optimally for low frequencies prefer upward FMs and neurons tuned to high frequencies fire more for downward FMs. Velocity selectivity, on the other hand, seems to vary systemically within each iso-frequency lamina and independently of tonotopy (Hage et al., 2003; Schreiner et al., 2000). It is therefore plausible that the auditory system can process the direction and velocity dimensions, only once it has processed the frequency dimension. Besides, our finding that learning frequency-dependent non-preferred direction is harder than learning the frequencypreferred direction combination discrimination, suggests a relationship with the physiology.

The temporal components are also extracted and represented throughout the auditory system (Barton et al., 2012; Brewer and Barton, 2016; Langner et al., 1997). Recent findings have found evidence for a topographical distribution of AM modulation rate that is orthogonal to the frequency tonotopic axis in AC and IC (Langner et al., 1997; Schreiner and Langner, 1988). However, there seems no organized topography of tone-pip modulation rate (repetition rate) (Kilgard and Merzenich, 1999). We found that the frequency dimension was on the same processing hierarchy as AM and tone-pip modulation rate dimensions, but only interacted with the later one, which therefore may relate to the physiology.

The auditory midbrain (IC) is the first station within the auditory pathway with neurons being selective for sound duration, sweep direction or sweep velocity (Brand et al., 2000; Hage et al., 2003; Kuo and Wu, 2012; Langner et al., 2002). It is believed that some sound features has already been extracted in IC (Malmierca, 2004; Winer and Schreiner, 2005). However, neuronal responses to different sound features are various in IC, i.e. almost all 
neurons show frequency selectivity but only half of them show sweep direction or velocity selectivity (Hage and Ehret, 2003), suggesting that the processes of feature extracting are hierarchical: from neurons that encode only one acoustic feature to neurons that encode multiple features that may be important for feature interaction. Thus, the hierarchical organization for low-level features we observed here may not be resulted from sequential feature extraction along different auditory stations, but due to the dynamic representation of each auditory feature in an early station, probably the IC.

\subsubsection{Methodology considerations}

To our knowledge, our experiment constitutes the first successful use of multidimensional generalization to infer the dimensional interaction and hierarchical organization of auditory information processing, which may yield important insights into the processes underlying auditory object processing and categorization. Multidimensional generalization has been studied almost exclusively with visual stimuli and mainly in humans and pigeons (Shepard, 1991; Soto and Wasserman, 2010). In agreement with these studies in the visual modality (Shepard, 1987; Soto and Wasserman, 2010; Warren, 1954), our results show that the shape of the multidimensional generalization gradient varies depending on the exact dimensions used in the task, indicating that the generalization relies strongly on the pre-wired topography of the system.

A concern is the fact that the salience of stimuli difference along each tested dimension may not be comparable. We did find that for the combination of frequency and direction dimensions, increasing the salience of the non-preferred dimension (direction) did not change generalization gradient but indeed improved discrimination performance. It might be necessary to test the other dimension combinations to check whether the effect of salience is the same for combination of equal-hierarchy dimensions.

It should be noted that our result may not be applied to complex natural sounds, like animal vocalization and human speech, whose processing is likely underlain by more complex neuronal mechanisms. For speech in particular, fluctuations in the temporal duration are common (Miller et al., 1986), but our auditory system is able to normalize the duration dimension to stably perceive speech (Newman and Sawusch, 1996). Thus the important role of sound duration may not hold, at least in the range of fluctuations that are typical of speech. 
To conclude, we found that the perception of acoustic dimensions can be segregated or integrated depending on the combination of dimensions, which relates to the physiology of the auditory system. We showed that processing of acoustic features is hierarchical organized. We observed that low-level dimensions were less or not at all generalized after discrimination learning and the ability to discriminate along these dimensions was more specific. 


\section{Chapter 3 Auditory learning modulates neuronal activity in the Inferior Colliculus}

\subsection{Introduction}

How sensory information is transformed into meaningful percepts and used to make decisions is one of the most fundamental questions in neuroscience. To achieve this transformation, sound features must be extracted and behavioral relevant information has to be interpreted. In the auditory system, the primary auditory cortex, like the visual cortex in the visual system, is commonly believed to process sound features of the acoustic stimuli (Chechik and Nelken, 2012; Chechik et al., 2006). During behavior, the organism has the ability to selectively attend to different aspects of the same sounds (Polley, 2006), which leads to the hypothesis that the auditory cortex can be selectively modulated by taskdemands. Indeed, a number of studies in awake behaving animals have shown that neural activity in the auditory cortex strongly depends on the behavioral states of the animal such as the level of task-engagement, arousal, locomotion, and learning, both in the auditory cortex (Bagur et al., 2018; Fritz et al., 2003; Otazu et al., 2009; Rodgers and DeWeese, 2014; Scheich et al., 2011; Schneider et al., 2018; Shimaoka et al., 2018), and the visual cortex (Dadarlat and Stryker, 2017; Dipoppa et al., 2018; Shimaoka et al., 2018; Vinck et al., 2015).

According to these results, the auditory cortex is viewed as the first station where behavioral relevant auditory objects emerge and are accessed during behavior. However, a recent study in head-fixed ferret have demonstrated that the receptive field of the inferior colliculus (IC) is also modified during behavioral engagement and adding the subcortical area to the hierarchy of processing behavioral relevant information (Slee and David, 2015). Indeed, the IC, as the first convergence station in the auditory circuit (Malmierca, 2004; Malmierca et al., 2002), receives feedback projections from the cortex (Winer, 2006), as well as innervations from the contralateral brain (Winer and Schreiner, 2005). The IC is, therefore, a good candidate as the interface between the periphery, the auditory cortex and other cortical areas, which can be modulated by behavior. So far, whether and how auditory learning and perception modulate activity in the auditory midbrain of freely moving animals is not well understood. 
To address this issue, we compared IC neuronal activity in freely behaving mice in different behavioral contexts: during behaviorally relevant sound exposure, during a puretone frequency discrimination task (conditioning) and during passive listening. We recorded the single unit and multiunit activity using individually moveable tetrodes implanted in the left IC. We examined the effect of behavioral contexts on spontaneous activity, sound-driven activity and overall response gain. We also addressed whether there was a persistent effect on neural activity in IC induced by behavior.

\subsection{Results}

\subsubsection{Pure-tone frequency discrimination task in the Soundterrace}

We first established a pure-tone frequency discrimination task in the Soundterrace which allows awake recording from behaving animals (Figure 0-4; see Methods). A series of trials was initiated by a mouse entering the corner of the Soundterrace. Each trial had duration of 13 to 17 seconds and a sound stimulus of a given frequency was presented from the speaker above the corner, indicating whether the mouse should nose-poke or not. The sound stimuli consisted of $30 \mathrm{~ms}$ pure tone pips that were repeated at $3 \mathrm{~Hz}$. Each nosepoke during the safe tone presentation was followed by an $8 \mu$ water-reward, while nosepoking during the conditioned tone presentation was punished with an aversive air-puff and no reward.

To establish and optimize the task, we first trained 8 non-implanted mice in the behavioral paradigm. The behavioral performance was quantified by calculating the daily fraction of trials with nose-pokes for each tone. To quantify discrimination, d' value, a standard measurement of discriminability adopted from signal detection theory, which reflected the separation between the mean of the correct target responses and the false alarms, was calculated. Mice were initially trained in the exposure phase in which only the safe tone, of $7000 \mathrm{~Hz}$ tone frequency, was presented. Mice showed an average baseline of $75 \%$, such that in $75 \%$ of the safe trials the animals nose-poked. After four days of exposure phase, conditioned trials, in which $14000 \mathrm{~Hz}$ conditioned tone was presented, were introduced. On average, mice reached good discrimination, defined by having d' value above 1, after two days of conditioning (Figure 3-1A). Animals' performance was stable after learning. They nose-poked in $88 \%$ of the safe trials and $30 \%$ of the conditioned trials, and had an average d' at 1.71. A three-way ANOVA for performance during conditioning day 3 to 5 revealed a main effect of trial type $(F(1,37)=153.93, p=0)$, but 
no effect of animals $(F(7,37)=1.99, p=0.081)$ or conditioning days $(F(2,37)=0.64, p=$ 0.53). Thus, our training paradigm allowed animals to learn the pure-tone discrimination relatively fast and stable.

A

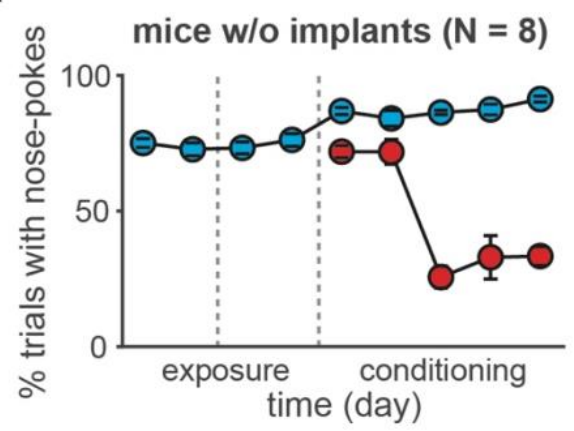

C

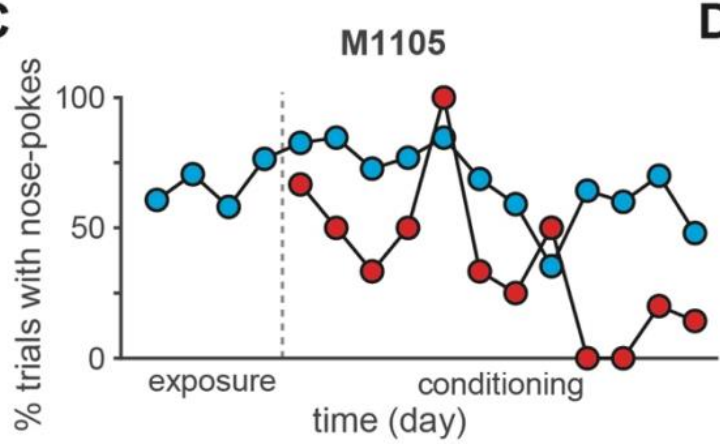

B

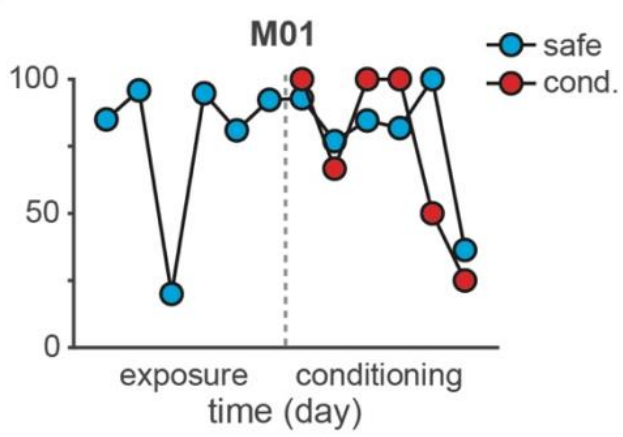

D

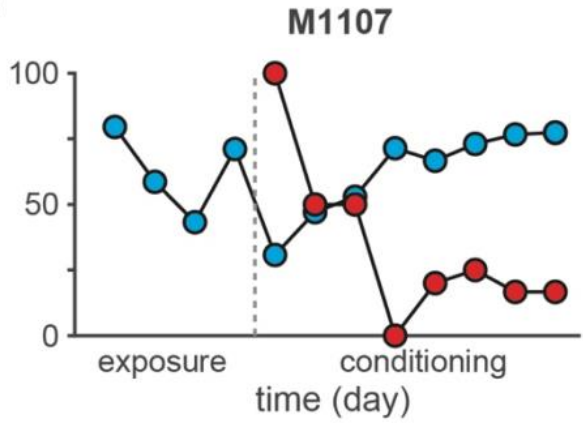

Figure 3-1 Pure-tone discrimination training in the Soundterrace

(A) Average daily performance for non-implanted mice expressed as the fraction of trials with nose-pokes for the safe (blue) and conditioned (red) trials. (B-D) Same as (A) for individual mice with chronic implants numbered as M01, M1105 and M1107, respectively.

To record the neural activity, including single-unit and multiunit (MUA) responses, we implanted 4 individually moveable tetrodes in the left inferior colliculus (IC) of C57BL/6j mice. After one week of recovery, implanted mice were water restrained for two weeks and its neural activity was recorded when the animal was freely moving in the arena of the Soundterrace. The aim was to optimize the quality of the recording, as well as to characterize the receptive field of the recording sites before behavioral training. The sounds used for subsequent discrimination task were chosen based on two criteria: the frequency distance between the to-be-discriminated tones was at least 0.75 octave; and more than half of the recording sites responded to either of the tones during the optimization period. The performance of mice with chronic implants during discrimination training is shown in Figure 3-1B-D. Compared to naïve mice, it took longer time for mice with chronic implants to reach good and stable discrimination: 8 days for mouse M1105 
and 3 days for mouse M1107 (Figure 3-1C, D; average d' across the last four conditioning days, $\mathrm{d}^{\prime} \mathrm{m} 1105=1.57, \mathrm{~d}^{\prime} \mathrm{m} 1107=1.62$ ). One mouse did not learn the task since it responded to the safe and conditioned tone similarly (Figure 3-1B; $\mathrm{d}^{\prime} \mathrm{m} 01=0.15$ ). Training was terminated when this mouse stopped nose-poking and made less than 5 visits in a half-hour session. On average, mice with chronic implants received about 50 trials per session. For each session, the activity of the same set of units was recorded during the active behavior (engaged) and during passive listening before and after behavior (passive). In the passive listening, pure tone pips with various frequency-intensity combinations were used to map the frequency-intensity tonal receptive field for each recording site.

\subsubsection{Task engagement increases spontaneous activity in IC}

To examine how neural activity in the IC depended on the behavioral state of the animal, we first compared the spontaneous activity when the mouse was passively listening with spontaneous activity when the mouse was actively engaged in the discrimination task (Figure 3-2). In agreement with previous findings in the auditory cortex (Rodgers and DeWeese, 2014; Scott et al., 2007), increase in spontaneous activity was observed in most of single units (Figure 3-2A, B; $n=39$ units, 2 mice; paired t-test, $p=0.0003$ ). For neural population activity, we found that most multiunits also showed increased spontaneous activity during task engagement (Figure 3-2C; $n=100,3$ mice; paired t-test, $p<0.0001$ ). To quantify the effect of task engagement across population, we calculated the modulation index as (Activity2-Activity1)/ (Activity2+Activity1). Negative modulation index indicates suppression in activity, and vice versa. The modulation index was positive for both singleunit and multiunit measurement, indicating that engaging in a discrimination task increased the number of baseline spikes (Figure 3-2D, F left). There was no change in spontaneous activity when the comparison was between the passive condition before and after task engagement (Figure 3-2D, E right; $p_{\mathrm{D}}=0.35, p_{\mathrm{E}}=0.11$ ), suggesting that the increase in spontaneous activity during task engagement may not be a result of non-stationary recording or thirsty level. 

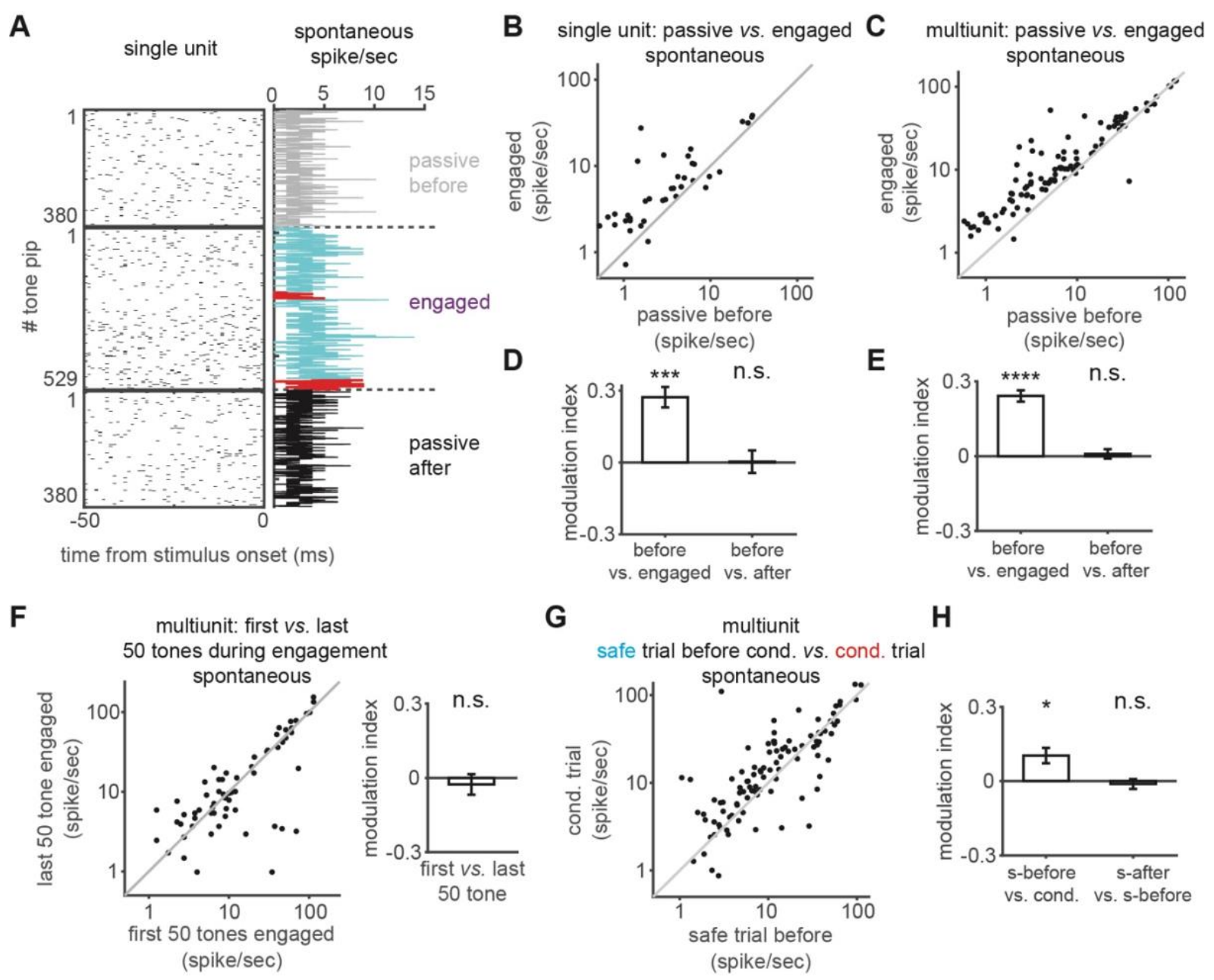

H

Figure 3-2 Task engagement increased spontaneous activity in IC

(A) Example raster plots (left) and PSTH (right) of single unit spontaneous responses preceding each stimulus onset for engaged (middle) and passive (top and bottom) conditions. Spontaneous activity recorded during the safe (blue) and conditioned (red) trial was marked respectively. (B-C) Population responses of single units and multiunits respectively. The data are presented as scatter plots comparing spontaneous activity in the passive listening before task engagement with activity in task engagement across population. (D-E) Modulation index calculated as (Activity2-Activity 1 )/ (Activity2+Activity1) for single unit and multiunit spontaneous activity, respectively. Spontaneous activity during engaged (left) and passive after engaged (right) condition was compared with activity during passive listening before task engagement. (F) Population responses (left) comparing multiunit spontaneous activity during the first with the last 50 engaged tone pips and modulation index (right). (G) Population responses comparing multiunit spontaneous activity during the safe trial before a conditioned trial with activity during the conditioned trial and modulation index $(\mathbf{H}-$ left). (H-right) Modulation index comparing multiunit spontaneous activity during the safe trial before with after a conditioned trial.

In some units, we observed a gradual increase in spontaneous activity during task engagement (Figure 3-2A). To verify if this was statistical significant at the population level, we compared spontaneous activity between the first and the last 50 tone presentation during the behavior (Figure 3-2F). There was no significant change overall (paired t-test, $p$ 
$=0.52$ ), indicating that the effect of task engagement on spontaneous activity was not accumulating throughout the task.

We next examined whether the spontaneous activity was influenced by the sound presentation during task engagement. Comparing activity during the safe trial immediate before a conditioned trial with activity during the conditioned trial, we found that spontaneous activity was transiently enhanced during conditioned trials (Figure 3-2G). There was no change in spontaneous activity between the safe trials before and after the conditioned trial.

Since we observed increase of spontaneous across the conditioning phase even when the mouse has not learnt the discrimination, it is possible that the increase was a result of other factors, like the change in locomotion between behavioral state. It has been reported by previous studies that locomotion influences neural activities in the sensory system (Dadarlat and Stryker, 2017; Niell and Stryker, 2010). And indeed, the locomotion of the animal was different between the passive and engaged conditions. When mouse was performing the task in the corner, it ambulated less than when it was in the arena. For this reason, we examined whether task-related changes in spontaneous activity can be observed during the exposure phase in which the animal was not required to discriminate sound stimuli because only the safe sound was presented. We found no significant change in spontaneous activity in the exposure phase comparing passive listening with task engagement (Figure 3-3A-D). It was confirmed with both single unit (Figure 3-3A, C; $n=$ 18 units, 2 mice; paired t-test, pbefore-engaged $=0.12$, $p$ before-after $=0.56)$ and multiunit (Figure 3-3B, D; $n=47$ units, 3 mice; paired t-test, pbefore-engaged $=0.15$, pbefore-after $=0.80$ ) measurements, indicating that the increase in spontaneous activity observed when the mouse was engaging in a discrimination task was not result from locomotion change, arousal, water reward, or purely sound-exposure. 

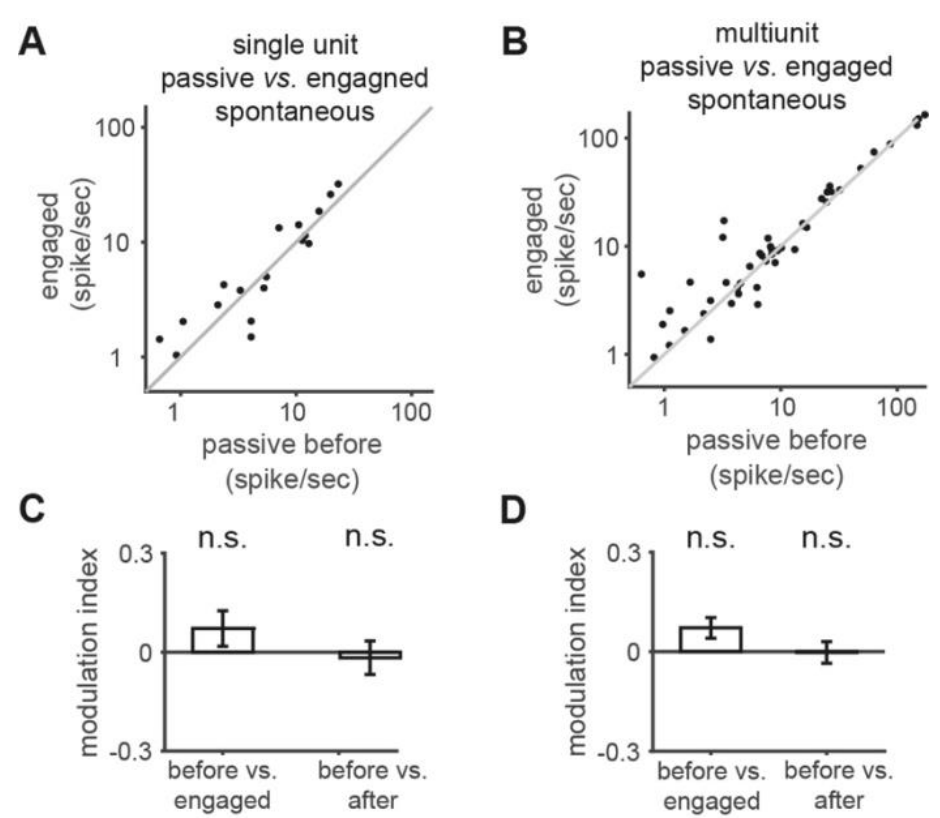

Figure 3-3 Spontaneous activity was not changed during exposure phase

(A-B) Population spontaneous activity of single units and multiunits respectively. (C-D) Modulation index. Spontaneous activity during engaged (left) and passive after engaged (right) condition was compared with activity during passive listening before task engagement.

In summary, engaging in an auditory discrimination task increased the spontaneous activity in the inferior colliculus. Our data rule out the possibilities that this increase was caused by non-stationary recording, arousal or locomotion. It may, therefore, be induced by an enhancement of attention to the frequency feature of the stimuli.

\subsubsection{Task engagement selectively suppressed the sound-evoked responses to the conditioned tone}

We next examined how task engagement modulated sound-evoked activity in IC. Interestingly, we observed single units that showed increased evoked responses, without baseline-activity subtraction, to the safe tone (Figure 3-4A), while the same unit showed decreased evoked responses to the conditioned tone (Figure 3-4D). A similar effect was also observed in multiunit measurements (Figure 3-4B, E). Due to insufficient responsive single units for population analysis, the statistical analysis was done using multiunit data. We found that most of the multiunits increased the evoked responses to the safe tone (Figure 3-4C; $n=75$ unit, 3 mice; paired t-test, $p<0.0001$ ), whereas, their responses to the conditioned tone were slightly suppressed (Figure 3-4F; $n=64$ unit, 3 mice; paired t-test, $p$ $=0.016)$. 

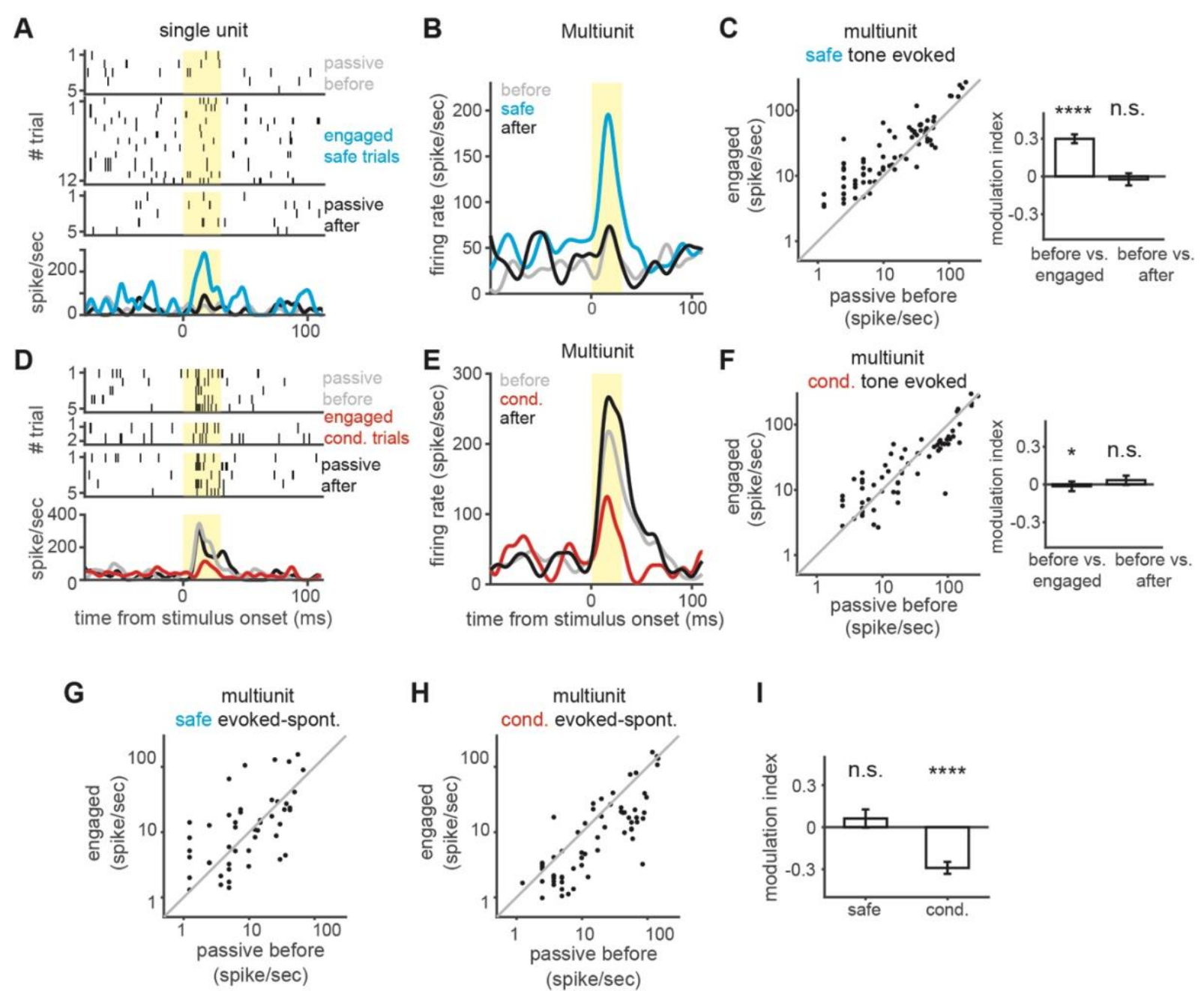

I

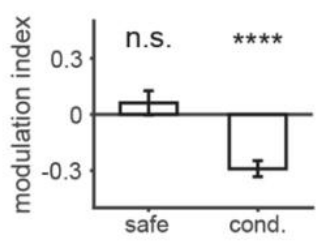

Figure 3-4 task engagement selectively suppressed sound-evoked activity

(A) Example raster plots (top) and PSTH (bottom) for single unit sound-evoked responses to the safe tone(yellow bar) during conditioning phase showing enhancement in the engaged condition (blue) relative to passive condition (grey and black). (B) Example PSTH of multiunit responses to the safe tone (yellow bar) during conditioning phase showing enhancement in the engaged condition (blue) relative to passive condition (grey and black). (C-left) Population evoke responses to the safe tone of multiunits. (C-right) Modulation index. Sound-driven activity during engaged (left) and passive after engaged (right) condition was compared with activity during passive listening before task engagement. (D-F) Same as (A-C) for responses to the conditioned tone. (G) Population baseline-subtracted evoke responses to the safe tone of multiunits. (H) Same as $(\mathbf{G})$ for responses to the conditioned tone. (I) Modulation index for comparison in $(\mathbf{H}, \mathbf{G})$, respectively.

We now took into account the enhancement of spontaneous activity described above and subtracted baseline activity from the evoked response. The baseline-subtracted evoked responses to the safe tone did not change overall (Figure 3-4G; paired t-test, $p=0.081$ ), while the baseline-subtracted responses to the conditioned tone remained suppressed (Figure 3-4H; paired t-test, $p<0.0001$ ). These changes suggest that the signal-to-noise 
ratio between sound-evoked and spontaneous activity decreased for both the safe and the conditioned tone, however, this decrease was more pronounced for the latter.

Taken together, we found that task engagement induced a robust suppression in evoked responses to the conditioned sound, but no overall change in evoked responses to the safe sound. This differential modulation resulted from changes in baseline to evoked activity relationship, such that for the safe sound both of them were enhanced while for the conditioned sound the spontaneous activity was enhanced and the evoke slightly suppressed.

\subsubsection{Global increase in gain and signal-to-noise ratio after a discrimination task}

Auditory experience has been shown to result in plasticity in adult auditory cortical and subcortical structures (see Irvine, 2018 for reviews). In the auditory cortical structures, long-term plasticity induced by auditory learning has been observed in a variety of associative learning procedures (Bieszczad and Weinberger, 2010; David et al., 2012; Engineer et al., 2004; Polley, 2006; Weinberger, 2007). Rapid task-related changes has also been observed in the cortex (Fritz et al., 2003). Short-term plasticity that lasted for a few hours have been reported in the subcortical auditory processing regions after classical conditioning, including the medial geniculate body (MGB) (Edeline and Weinberger, 1991) and inferior colliculus (IC) (Gao and Suga, 1998). Recently, our own lab found that learning can also induce long-term plasticity, in the form of a widespread increase of sound-evoked activity, in IC (Cruces-Solís et al., 2018). We therefore examined whether modulation of neural activity induced by task engagement was accompanied by longlasting plasticity occurring after the task. To differentiate the effect of learning and task performing, we separated the units recorded during learning from those recorded when the mouse had learned the task.

For multiunits recorded when the mouse had already learnt the task, population analysis revealed significant enhancement in overall absolute sound-evoked activity (Figure 3-5A, D-overall evoked; paired t-test, $p=0.018$ ) and spontaneous activity (Figure 3-5B, Dspontaneous; paired t-test, $p=0.028$ ). The stronger modulation in overall sound-evoked activity, therefore, led to an increase in overall signal-to-noise ratio (SNR; Figure 3-5C, DSNR; paired t-test, $p=0.009)$. To further understand whether this was a widespread effect across the receptive field of each unit, we calculated the tuning curves as normalized 
average firing rate across intensity evoked by stimuli of $30 \mathrm{~ms}$ duration as a function of distance in octave to its best frequency (BF). We found that the increase in overall soundevoked activity was homogeneous distributed across frequencies (Figure 3-5E), indicating a widespread enhancement of evoked activity. Most of the multiunits had a BF between 7 $\mathrm{kHz}$ (the frequency of the safe tone) and $14 \mathrm{kHz}$ (the frequency of the conditioned tone; Figure $3-5 \mathrm{~F}$ left). The BF tended to shift upwards in the direction towards the conditioned frequency, but the shift lacked statistical significance (Figure 3-5F; paired t-test, $p=0.17$ ). We further characterized the relationship between the receptive field and the modulation index of the responses. If the modulation occurred mainly in a sub-group of neurons that responded to the task-related stimuli, we would expect to observe a decrease in modulation index with the dissimilarity between the BF of the neuron and the task frequencies. However, there was no correlation between the modulation index and this dissimilarity (Figure 3-5G; correlation coefficients, $r=0.12, p=0.47$ ). Therefore, task engagement induced an overall increase in response gain and signal-to-noise ratio, which was not related to the receptive field properties of IC neuron.

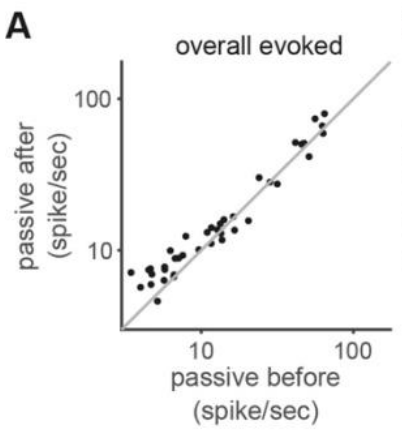

E

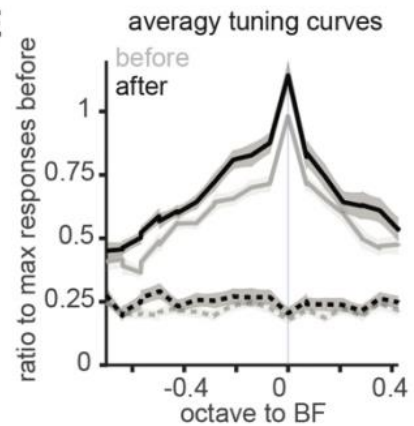

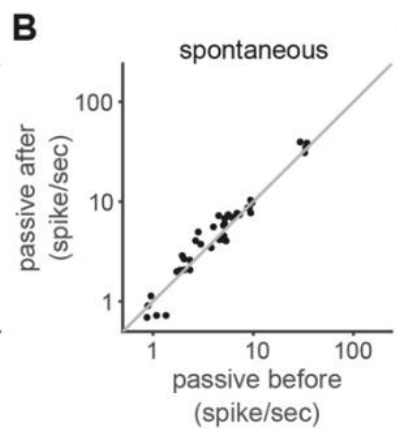

$\mathbf{F}$

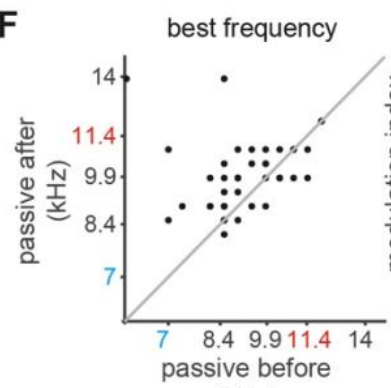
$(\mathrm{kHz})$
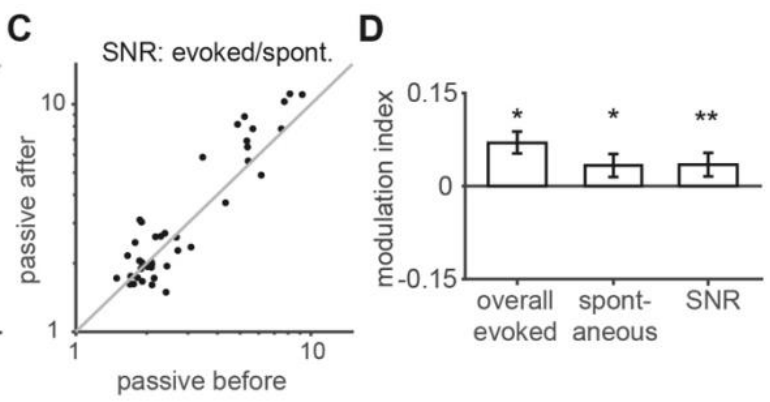

G
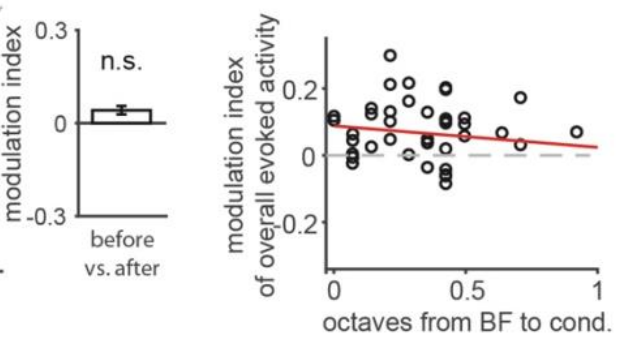

Figure 3-5 Increase in global gain and signal-to-noise ratio induced by engaging in a discrimination task

(A-C) Population analysis for multiunit absolute overall evoked activity, spontaneous activity and signal-to-noise ratio showing increased gain in the passive conditions after the task engagement relative to the passive condition before. (D) Modulation index for comparisons in (A-C) respectively. (E) Normalized average tuning curves for multiunit responses as a function of frequency distance to its BF in passive listening before (grey) and after (black) the task engagement. 
Absolute evoked activity and spontaneous activity was presented as solid line and dash line respectively. Tuning curves were normalized to the peak evoked responses in passive listening before task engagement. (F) Population analysis (left) and modulation index (right) for BFs comparing two passive listening conditions. (G) The (passive before versus after) modulation index plotted for each multiunit as a function of frequency distance between its BF to the conditioned frequency. There was no correlation between those two (correlation coefficients, $r=0.12, p=0.47$ ).

For units recorded during conditioning, when the mouse had not yet learnt to discriminate, there was no change in either overall sound-evoked activity or spontaneous activity (Figure 3-6A-C; paired t-test, pevoked $=0.73, p_{\text {spont. }}=0.31$ ). Thus, in our task, learning did not elicit persistent change in sound-evoked or spontaneous activity in IC.
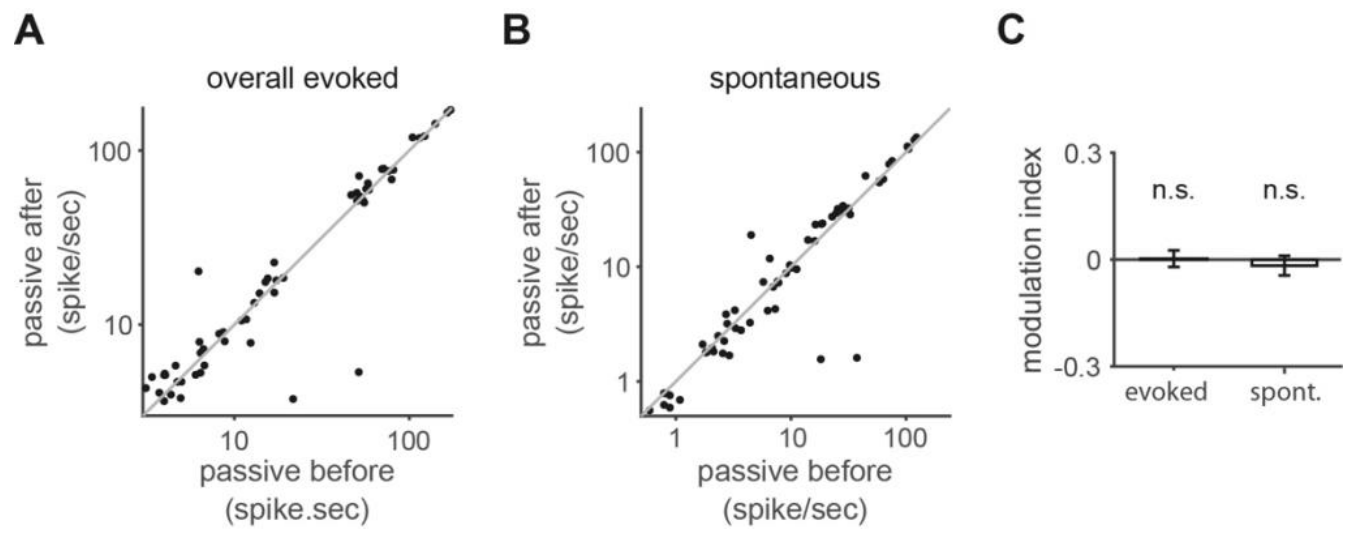

Figure 3-6 Lack of changes in global gain evoked by discrimination training

(A-B) Population analysis for multiunit absolute overall evoked activity and spontaneous activity showing no change in gain in the passive conditions after discrimination training relative to the passive condition before. (C) Modulation index for comparisons in (A, B) respectively.

Sound exposure can induce long-lasting response gain changes in the inferior colliculus of anesthetized animals (Cruces-Solís et al., 2018). To test whether associating one sound with reward elicited persistent change in response gain, we performed the same analysis for the exposure phase. Here, no overall change in sound-evoked activity was observed in the IC of freely moving mice (Figure 3-7A-B). 
A

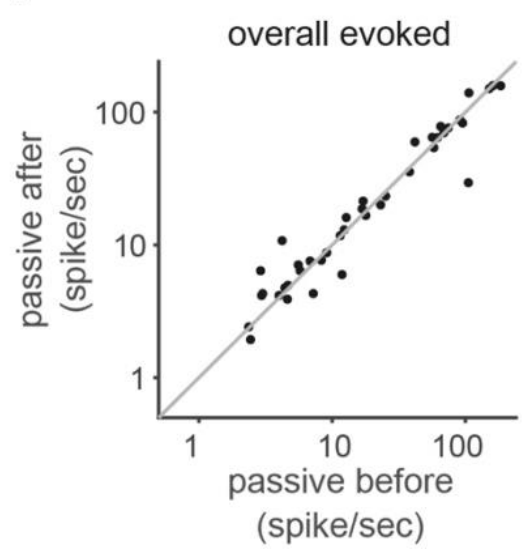

B

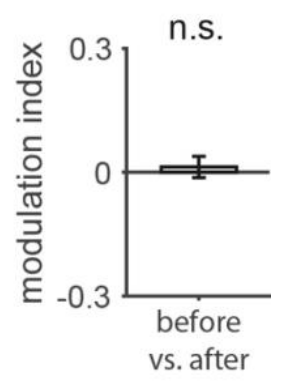

Figure 3-7 Lack of changes in global gain evoked by acoustic stimuli associated with reward

Population analysis (A) and modulation index (B) for multiunit absolute overall evoked activity showing no change in the passive conditions after exposure relative to the passive condition before.

Taken together, successfully performing an auditory discrimination task elicited persistent change in global gain and signal-to-noise ratio, which was not dependent on learning or sound exposure.

\subsection{Discussion}

In this study, we found that engagement in a discrimination task modulated neural activity in the IC of freely behaving mice in a state-dependent manner. Comparing responses during active discrimination with responses during passive listening, we found that task engagement reshaped the relation between the spontaneous and sound-evoked activity, with an increase in spontaneous activity but a bidirectional modulation of soundevoked activity. For stimuli paired with reward, an enhancement of evoked responses together with the increase in spontaneous activity led to no overall change in baselinesubtracted evoked responses. However, for stimuli associated with punishment, a weak suppression of evoked responses together with the increase in spontaneous activity resulted in a robust suppression of the baseline-subtracted evoked activity. Comparing responses during passive listening before and after task-engagement, we found that the task-related plasticity was accompanied by a persistent change in global gain during subsequent passive listening. 


\subsubsection{What is the cause of the effect associated with task engagement?}

Increased arousal is a good candidate to explain the changes during task engagement. It has been shown in many studies that the increase in arousal increases cortical excitability, including the spontaneous and evoked activity (Duque and Malmierca, 2015; Edeline et al., 2001; Otazu et al., 2009). In our results, the effect of increased arousal may contribute to the increase in spontaneous activity and absolute evoked responses to the safe tone. However, this may not explain the selective suppression effect on evoked responses to the conditioned tone. Thus, at least some components of task-related plasticity observed in our study might be independent of the level of arousal. To dissociate the effect of arousal and task-engagement, it would be useful to measure arousal state during recording. Pupil diameter could be used as an index. However, it might be difficult to conduct in freely moving animals.

Since mice in our study were not restrained, one interpretation of our results is that the change in locomotion during behavior induces elevation in neural firing (Dadarlat and Stryker, 2017; Niell and Stryker, 2010; Vinck et al., 2015). However, the exposure phase shared similar locomotion pattern as the conditioning phase, and yet it did not lead to changes in activity suggesting that the task-engagement effect is not a result of locomotion change. This result also rules out the effect of reward (Brosch et al., 2011; Shuler and Bear, 2006), sound exposure (Cruces-Solís et al., 2018), or expectation (Rummell et al., 2016; Schneider et al., 2018).

The differential modulation of evoked responses to the safe and conditioned tone may be explained by selective-attention mechanisms. Selective attention is reported to differentially modulate neural responses during auditory discrimination task (Edeline and Weinberger, 1993; Fritz et al., 2005, 2007), which maximizes the difference in neural responses to the to-be-discriminated sounds to achieve effective discrimination. A recent finding further shows that the differential changes are depended on the behavioral readout that is associated with each stimuli (David et al., 2012). For stimuli indicating a 'go' behavior, responses to this stimulus tended to decrease, whereas response to stimuli indicating a 'no-go' behavior was increased. This may explain why we observed both enhancement and suppression in IC evoked activity induced by discrimination task engagement, but only suppression was observed previously in auditory detection task (Slee and David, 2015). However, what we observed followed an opposite pattern that the responses to the 'no-go' stimuli were suppressed. One interpretation is that it reflects a differential modulation on surrounding neurons that fire suboptimal to the target stimulus 
(David et al., 2012). Because we optimized the stimuli such that both of them elicited response in more than half of the tetrodes, most of the neurons from which we recorded were not optimally driven by the safe or the conditioned stimuli, but were tuned to frequencies between them (Figure 3-5F). This can be addressed by recording from neurons cover a wider range of receptive fields in the future.

\subsubsection{Mechanisms of modulation induced by task engagement}

Our central finding that engaging in an auditory discrimination task selectively suppresses responses to the conditioned tone was partially the result from an increase in population-level spontaneous activity. Spontaneous activity has been shown to influence the sensory-evoked activity (Bennett et al., 2013; Ferezou and Deneux, 2017; Haider et al., 2007; Seger and Peterson, 2013) and therefore play an active role in sensory processing (Carcea et al., 2017; Luiz et al., 2017; Xu et al., 2012). Carcea et al. (2017) found a decrease in spontaneous activity after self-initiation of an auditory task, which was important for sound perception as suggested by optogenetic disruption. Experience-related change in spontaneous activity has been reported in sensory system (Bagur et al., 2018; Otazu et al., 2009; Xu et al., 2012). Particularly, it is been observed in the visual cortex that training rats with repeated visual stimuli elicited recalls of the same sequential firing pattern in the spontaneous activity during the followed resting period (Xu et al., 2012). Therefore, spontaneous activity may reflect an internal representation. Here, we show modulation of spontaneous activity may also act as a mechanism attributing to the taskspecific effect on the IC activity. Previously, we found changes in spontaneous activity in the IC of exposed, not conditioned, animals that resulted in local effects on signal-to-noise ratio (Cruces-Solis et al., 2018). Thus, regulation of spontaneous activity seems to be a common mechanism underlying sensory gating in the IC of behaving animals.

The neural mechanism underlying the changes in IC during task engagement is not yet clear. The dense corticocollicular projection (Winer and Schreiner, 2005) which has been shown to have modulatory effect on IC activity (Suga and Ma, 2003; Zhang et al., 1997), may serve for these changes. However, the similar level of task-related plasticity in the lateral/dorsal cortex (DCIC) of IC and the central nucleus (CNIC) contradicts this hypotheses (Slee and David, 2015). Since anatomically the Corticocollicular projection to DCIC is denser than CNIC, one would expect to observe stronger changes in the former one. 
The overall change in spontaneous activity may suggest the engagement of the neuronmodulatory system. The cholinergic, noradrenergic, serotonergic innervations of IC (Ayala and Malmierca, 2015; Klepper and Herbert, 1991; Nevue et al., 2016; Schofield, 2010) have been shown to influence sensory coding (Ayala and Malmierca, 2015; Gittelman et al., 2013). In addition, the differential modulation in responses to the safe and conditioned tone Corticofugal projection could be resulted from a top-down control from the frontal cortical areas, of which activity has been shown to be influenced by decision.

In classical conditioning, many stations, such as IC and cochlear nuclei, in the auditory system receive direct US somatosensory projections (Poremba et al., 1997). It is possible that the changes during conditioning may be attributed to the perception of the aversive airpuff. Then one would expect to see stronger modulation during training but weaker effect after learning, since then the mouse receives fewer air-puffs after learning. However, when we analyzed the effect of task engagement separately for those two stages (before vs. after learning), we did not observe differential modulation pattern.

\subsubsection{The persistent plasticity in periods immediately following behavior}

We found that task engagement induced persistent change in IC global gain and response signal-to-noise ratio in periods immediately after. It is worth noting that the modulation pattern was different from what we observed during the task in which the modulation in evoked activity was stimuli-specific. As suggested by Slee et al, this may be caused by a sustained high level of arousal or reflected a task-demanded memory trace (Slee and David, 2015). If we assume that the level of arousal during the exposure phase, the learning and post-learning conditioning phase are similar, since they all required the mouse to actively move into the corner and nose-poke to get water, we should observe similar effect of arousal on IC. However, the persistent plasticity was only observed after mouse reached good discrimination, thus ruling out the effect by change in arousal.

It is interesting to note that we did not observe persistent change during learning, which contradicts previous finding that learning can induce plasticity in IC using classical conditioning paradigms (Gao and Suga, 1998). One interpretation is that it may result from different training paradigm. In classical conditioning, learning usually occurred after a single trial training (Bouton and Moody, 2004). However, in our discrimination task, the mice learned the association between each stimulus and its valence, reaching a stable behavioral performance level in which they could respond to each stimulus accordingly, at 
least after two days of training. For both mice with and without chronic implant, the change in performance was not gradual, but occurred very sudden. The neural basis for the changes underlying this longer-term training may be distinct from the short-term training in classic conditioning.

\subsubsection{Technological consideration}

We've obtained nice single-unit recordings using chronic implants. However, the overall quality of spikes, signal-to-noise ratio, and stability of chronic recording was not optimal. The spikes were not as large and well isolated as those obtained from acute recordings using the same electrodes. And quality of spikes decreased with time from implantation. Usually after a month of recording, it was very hard to record spikes with good SNR. We reason that this might have been partially caused by the inflammation around the implanted electrodes and the growth of scar tissue around implanted tetrode tips. Similar issues have been reported by others using the same technique in the auditory system of mice. Therefore, it may be worth to move to record from head-fixed mouse instead of chronic implants in the future.

Taken together, our data are consistent with previous findings in head-fixed ferrets where IC responses to a target sound were suppressed (Slee and David, 2015). In our task, responses to the target sound were diminished relative to baseline as a result of increased spontaneous activity (Figure 3-4). Previously, the lab found changes in spontaneous activity in the IC of exposed, not conditioned, animals that resulted in local effects on signal-to-noise ratio (Cruces-Solis et al., 2018). While the function of the task-related suppression is unclear, regulation of spontaneous activity seems to be a common mechanism underlying sensory gating in the IC of behaving animals. The IC is, therefore, sensitive to the relevance of sound and to task-performance. 


\section{Chapter 4 Acoustic consequence of movement is the key factor for IC plasticity induced by relevant sound exposure}

\subsection{Introduction}

Perception relies on actively generating predictions of the brain's input based on innate or learnt knowledge (Schröger et al., 2015). The coding of predictive information depends on forming associations between two events, such as a sound stimulus and a foot shock. How behavioral outcomes can be predicted by a sensory stimulus has been extensively studied in different sensory modalities, including auditory (Irvine, 2018a; Soto et al., 2014; Weinberger, 2007). Learning of predictions can lead to long-lasting plasticity in the auditory cortex. However, how a sensory stimulus can be predicted by a self-generated event, i.e. pressing a button and the doorbell ringing, is not well understood. Studies in human and a variety of animals have shown that sensory responses to predictable selfgenerated sensory event are attenuated compared to external sensations (Blakemore et al., 1999; Eliades and Wang, 2003; Phillips et al., 2017; Rummell et al., 2016; Sato, 2008), which in turn changes one's perception, i.e. we can't tickle ourselves. Studies on movement-related auditory stimulus further show attenuation of self-initiated sounds in the auditory cortex (Carcea et al., 2017; Rummell et al., 2016; Schneider et al., 2018). Little is known whether the attenuation also followed by long-lasting plasticity in the auditory system.

In a recent study from the lab, mice were kept in a natural environment, the Audiobox (TSE, Germany), and were exposed to sounds that were played as long as the mouse was inside the water-corner (Cruces-Solís et al., 2018). The exposure sound was, therefore, context-specific since it only occurred in the corner, water-associated since the corner was the only place where water was available, movement-predictable since entering the corner triggered the sound, and temporally sparse since visits to the corner were typically more than a minute apart. This type of exposure resulted in a long-lasting change in neuronal response gain and frequency coding in the inferior colliculus of anesthetized mouse. Sound-evoked response was increased in a frequency-unspecific manner. The BFs of neurons along the dorsal-ventral axis shifted towards higher frequencies, resulting in a global shift in tonotopy. A different pattern of plasticity was observed in IC of mice that 
had been exposed to a sound with the same temporal pattern but randomly in the homecage of the Audiobox (random group). The control, the random and the normal exposure group differed in sound exposure, water presence, and movement-predictability. Yet, because of the complexity of the exposure context it is important to further understand were the predictability of the sound comes from and the importance of the water presence in the corner. Movement-related attenuation has been found to influence neuronal responses in multiple stations of the auditory pathway (Eliades and Wang, 2003; Singla et al., 2017), including the inferior colliculus (Suga and Shimozawa, 1974). Therefore, we hypothesized that the plasticity in the IC after this sound exposure was mainly triggered by the movement-sound association.

Here, we examined the effect of three possible factors, water association, contextspecificity, and movement-predictability, on plasticity induced by sound exposure in the Audiobox. Following the same experimental design as in Cruces-Solis et al. (2018), we studied how response gain and frequency coding were changed by manipulations in each of the above factors. Besides, we investigated how sound exposure and self-initiation modulate neural activity in the inferior colliculus of freely behaving mouse.

\subsection{Results}

To dissociate possible factors that may induce IC plasticity, we exposed five groups of mice in the Audiobox with different sound exposure paradigm (see Method; Figure 4-1A, B). It is important to note that none of the animals were conditioned, but only exposed. The "control" and "normal exposure" group were trained the same as in the previous study of our lab, i.e. the control group lived in the Audiobox for the same length of time as other groups without hearing $16 \mathrm{kHz}$ tone pips coming out of a speaker and the normal exposure group heard a fixed $16 \mathrm{kHz}$ tone for the duration of each visit to the water corner (CrucesSolís et al., 2018). The "no-water exposure" group was exposed to a $16 \mathrm{kHz}$ tone as the normal exposure group, but differing from all other groups they had access to water in the food area. The "unpredictable exposure" group heard a pure tone of $16 \mathrm{kHz}$ in only $80 \%$ of the visits and in these visits, the sound presentation started randomly 5 to 25 seconds after the mouse entered the corner. Therefore, the timing of sound exposure was not triggered by the animal and could not be predicted. The last group, "entry-only exposure" group, heard a fixed pure tone of $16 \mathrm{kHz}$ only during the first 3 seconds after entering the corner. For this group, sound exposure was predictable, since it was only triggered by the animal 
movement. Then, we acutely recorded from the left inferior colliculus of anesthetized animals that had been in the Audiobox for 9 to 15 days (exposed to sounds for 6 to 12 days, since the first 3 days in all groups involved habituation to the Audiobox without sound exposure).

In this set of experiments, electrophysiology was performed acutely after sound exposure in the Audiobox. To study exposure-triggered plastic changes as they happened we used a different approach. In another experiment, we recorded from freely behaving animals that were undergoing sound exposure in a home-build shuttle box or doing passive listening in their home-cage (see Method; Figure 4-7A; Figure 4-8A). We compared IC activity during passive listening in the home cage and when it was exposed to sound in the shuttle box. We then compared ongoing activity in IC during predictable sound exposure with one during unpredictable (random) sound exposure. 

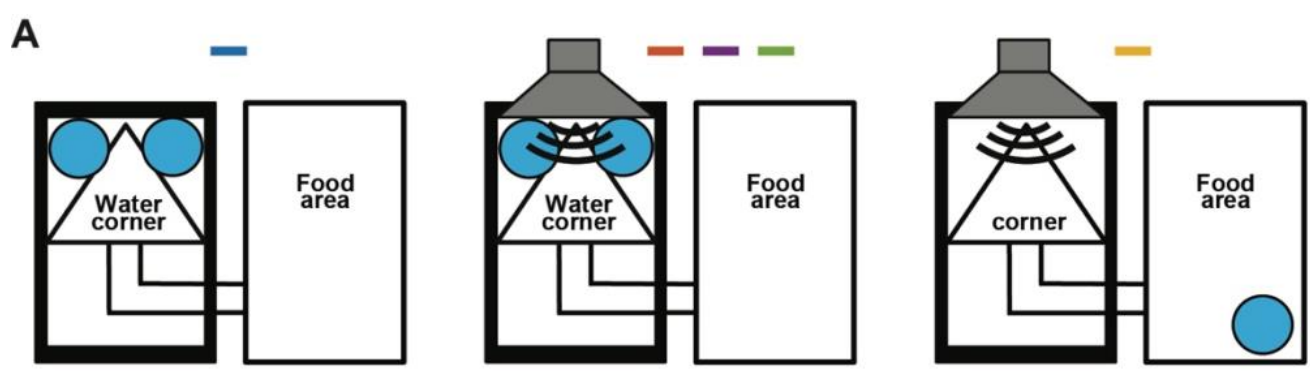

B

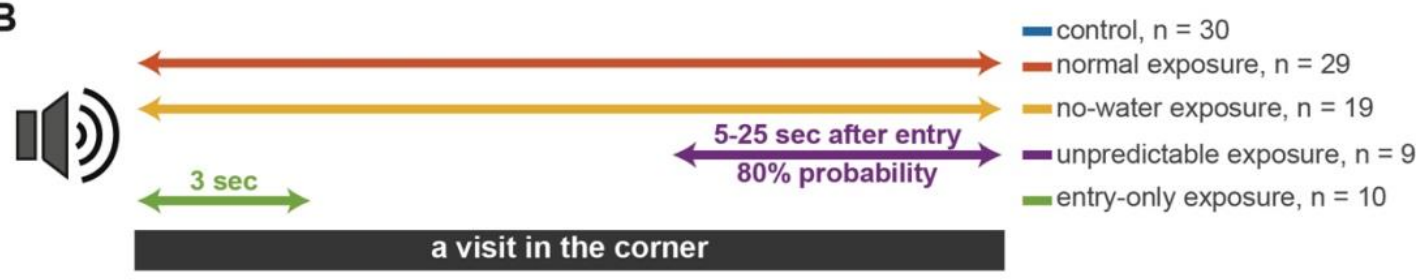

C

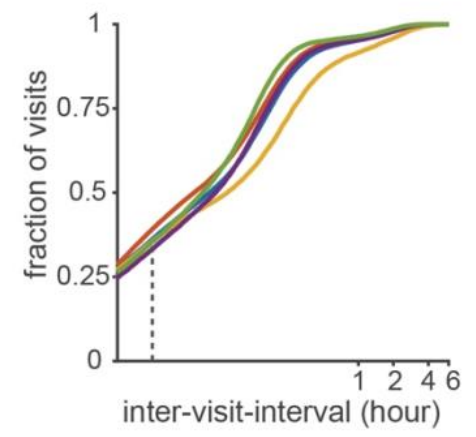

$\mathbf{E}$

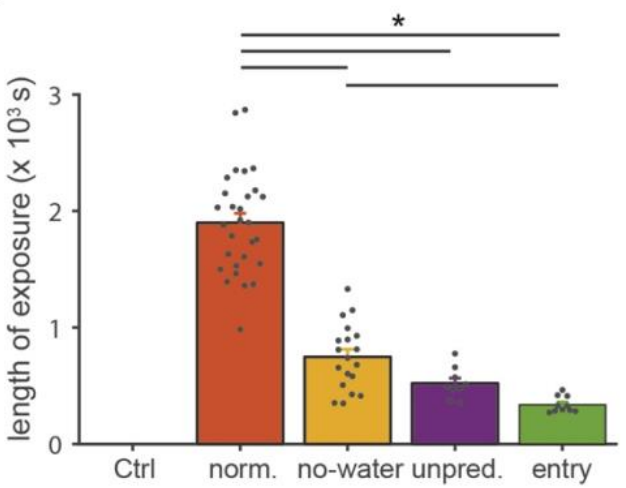

D

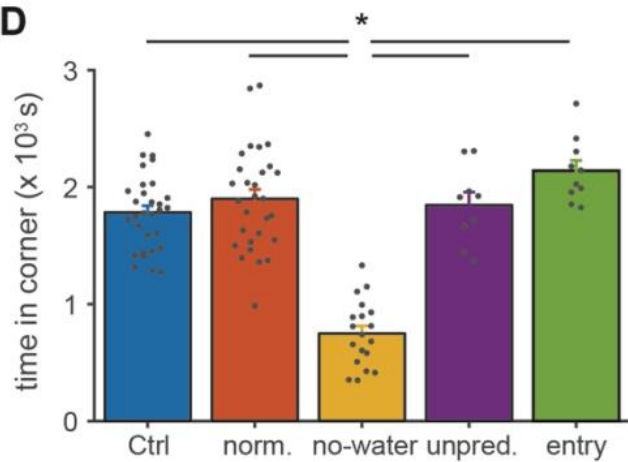

$\mathbf{F}$

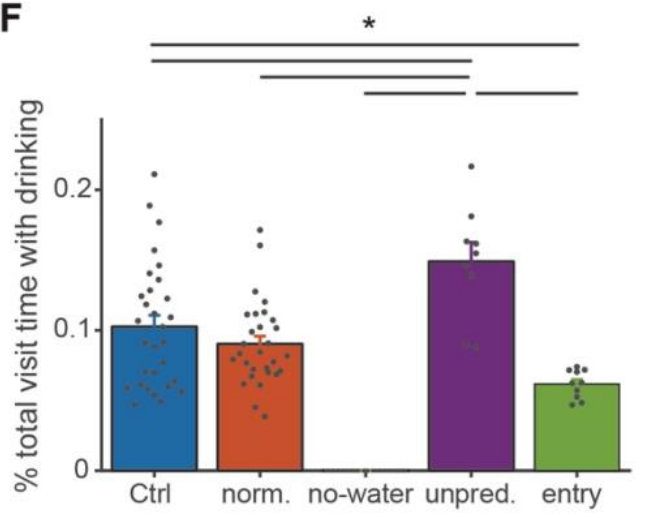

Figure 4-1 Sound exposure in the Audiobox

(A) Schematic representation of the Audiobox. Color bar on top of each scheme indicates the groups of mice. Food was available in the food area. Water was available in the corner (except nowater exposure group) or in the food area (no-water exposure group). (B) Schematic representation of sound exposure protocol. Sound exposure occurred in the corner for the duration of each visit (normal and no-water exposure group), with a silent interval of random length after entering the corner in $80 \%$ of the visits (unpredictable group), only for 3 seconds after entering the corner (entry-only exposure group), or not at all (control group). (C) Cumulative distribution of the intervisit-interval. The dotted lines indicate the fraction of visits with less than 1 minute inter-visit interval. (D) Average daily time spent in the corner of individual mice. The no-water group spent significant lower time in the corner daily (ANOVA, $F(4,92)=41.82, p<0.0001$. Corrected pair comparison, $\mathrm{p} *<0.05$ ). (E) Average daily duration of sound exposure of individual mice (ANOVA, $F(3,63)=88.66, p<0.0001$. Corrected pair comparison, $\mathrm{p} *<0.05)$. (F) Average daily percentage 
of total visit time spend on drinking (ANOVA, $F(3,74)=9.96, p<0.0001$. Corrected pair comparison, $\mathrm{p} *<0.05)$.

\subsubsection{Sound exposure in the corner elicits IC plasticity independent of water association}

We first replicated the main groups of a previous study in the lab (Cruces-Solís et al., 2018) and compared a control group with a group that received 'normal exposure'. Consistent with previous data, sound exposure for the duration of each visit (normal exposure) had no effect on the animals' behavior. Compared with control animals, mice in the "normal exposure" group show comparable inter-visit-interval (Figure 4-1C, blue vs. red), daily time spent in the corner (Figure 4-1D, blue $v s$. red) and average fraction of drinking time while in the corner (Figure 4-1F, blue vs. red). It is worth noting that for both control and normal exposure group, about $90 \%$ of the time when the mouse was in the corner it was not drinking water.

Since our main concern was that plasticity induced by normal exposure might have been dependent on the association between the water and the sound, since both were present in the corner, we also exposed mice in the Audiobox with $16 \mathrm{kHz}$ sound exposure for the duration of each visit but with no water in the corner (no-water exposure). Behaviorally, even though there was no water, mice still visited to the corner out of curiosity. As expected, their visits were temporally sparser and the total time spent in the corner was shorter than in the normal exposure group (Figure 4-1C, D, red vs. yellow).

After exposure to $16 \mathrm{kHz}$ sound for 6-12 days, we acutely recorded from the left inferior colliculus of anesthetized animals. We recorded multiunit activity using multielectrode arrays with 16 sites separated by $50 \mu \mathrm{m}$. The electrode was dorsoventrally inserted in IC along the tonotopic axis. Each mouse had two recording sessions: one with the top recoding site on the dura, and another one with the top recoding site at a depth of $500 \mu \mathrm{m}$. Therefore, the whole recording covered a range of depths between 0 and $1250 \mu \mathrm{m}$ (see Method; Figure 0-9). Depths more superficial than $100 \mu \mathrm{m}$ were considered to be putative dorsal cortex based on their distinct physiology (Barnstedt et al., 2015). The remaining was considered to be in the central nucleus. The tonal receptive field (TRF) was characterized for each recording site by presenting $30 \mathrm{~ms}$ pure tones with different frequency-intensity combinations.

The plastic changes we observed in the normal-exposed group replicated those of Cruces-Solis et al. (Cruces-Solís et al., 2018). An increase in sound-evoked responses was 
evident in the tuning curves comparing normal-exposure with control animals (Figure 4-1A; blue $v s$. red). We show tuning curves at a depth of 400,650 , and $900 \mu \mathrm{m}$, as $650 \mu \mathrm{m}$ was the depth with a best frequency (BF) of $16 \mathrm{kHz}$ for control animals. As before the increase was not limited to the depths that best responded to the exposed frequency but was, instead, frequency-unspecific.

The pattern was similar for animals exposed in the corner when water was only accessible in the home-cage. Taking the water out of the corner did not abolish the increase in responses induced by sound exposure (Figure 4-2A), suggesting the association between the $16 \mathrm{kHz}$ exposure sound and having access to water was not the cause of change in response gain. Quantification of average evoked response across tone frequencies confirmed that the enhancement of evoked responses in both normal and no-water exposure animals relative to control was homogenous along the IC tonotopic axis (Figure 4-2B). A two-way ANOVA revealed a main effect of group $(F(2,763)=21.47, p=0)$ and a main effect of depth $(F(25,763)=19.15, p=0)$, but no group $\mathrm{x}$ depth interaction $(F(50$, $763)=0.38, p=1)$. We found the evoked responses were stronger in recordings at higher depths (Figure 4-2B).

Experience in rodents suggests that increasing the probe-shank diameter from 20 to 200 $\mu \mathrm{m}$ results in a rapidly decrease in the unit recording quality, which may be caused by compression or damage to the dendrites of the surrounding neurons (Claverol-Tinture and Nadasdy, 2004). The multichannel electrode array (A1x16-3mm-50-177, NeuroNexus) used in our study has an uneven distribution of shank diameters that is $33 \mu \mathrm{m}$ at the tip and gradually increased to $123 \mu \mathrm{m}$ at the top site. The sudden drop in evoked response from 750 to $800 \mu \mathrm{m}$ could also be explained by this (Figure 4-2B), since the recordings from 800-1150 $\mu \mathrm{m}$ depths was using the top panel of the probe that had wider shank diameter. Nevertheless, as all the recording from different animals was done in the same way, the change in recording quality with depth could not explain our finding that sound exposure in the corner elicited homogeneous enhancement in sound-evoked responses along the IC tonotopic axis and this increase did not depend on water association. 

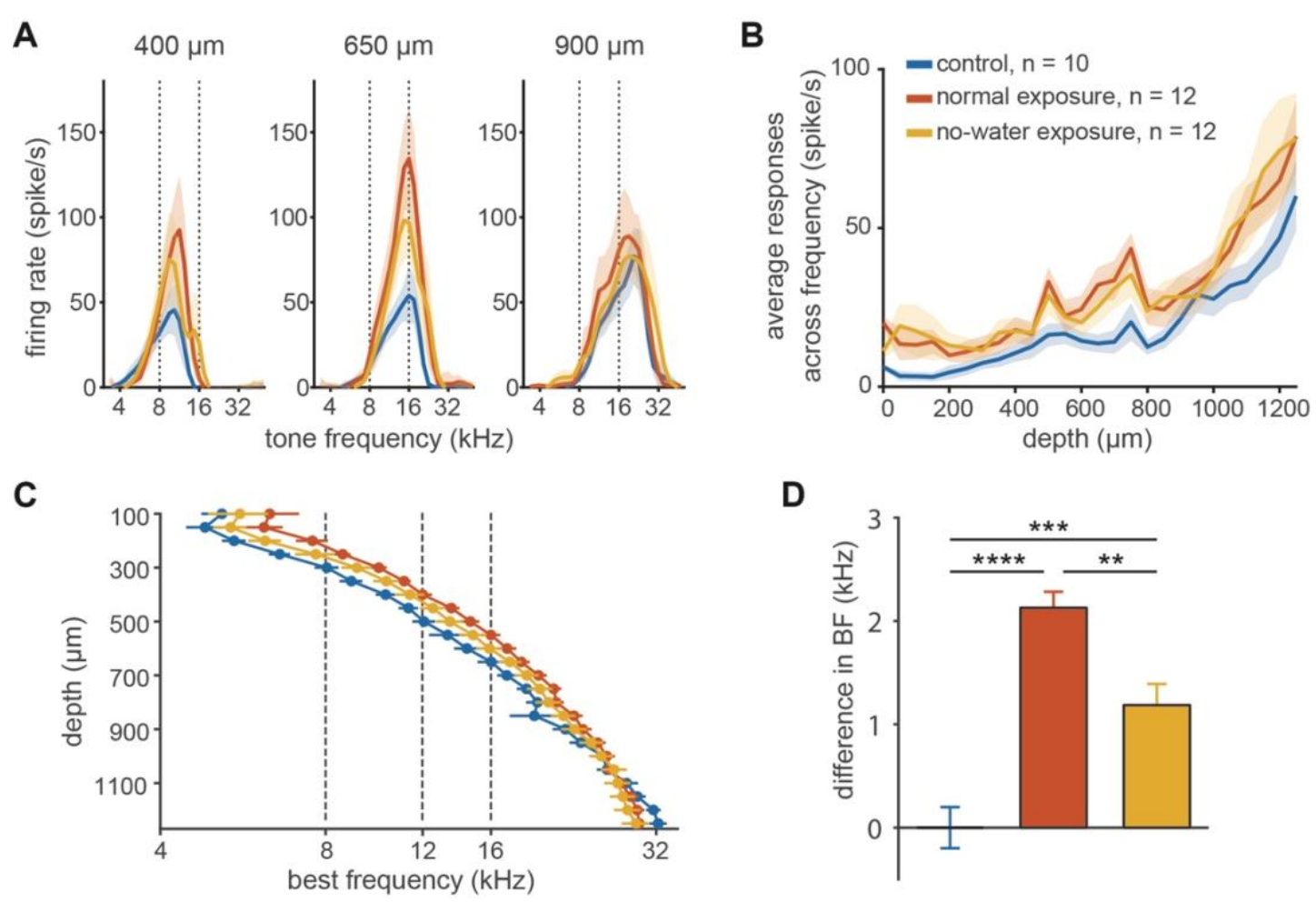

Figure 4-2 Plasticity induced by sound exposure was independent of water association

(A) Average tuning curves of sound-evoked activity at $70 \mathrm{~dB}$ for depth of 400,650 and $900 \mu \mathrm{m}$ in IC for control (blue), normal exposure (red) and no-water exposure (yellow) group. (B) Average evoked responses across frequency at $70 \mathrm{~dB}$ as a function of recording depth. The normal exposure and no-water exposure group showed stronger evoked response relative to the control group. A two-way ANOVA revealed a main effect of group $(F(2,763)=21.47, p=0)$ and a main effect of depth $(F(25,763)=19.15, p=0)$, but no interaction $(F(50,763)=0.38, p=1)$. Multi comparison confirmed significant difference between control and exposed groups ( $p_{c t r l, \text { norm. }}=0, p_{c t r l}$, no-water $=0$, pnorm., no-water $=1)$. $(\mathbf{C})$ Average best frequency $(\mathrm{BF})$ as a function of depths (two-way ANOVA, group $F(2,761)=6.88, p=0.001$, depth $F(25,761)=211.4, p=0$, interaction $F(59,761)=0.85, p$ $=0.77)$. (D) Average difference in BF with respect to the average BF of control group across the depths between 100 and $900 \mu \mathrm{m}$ (ANOVA, group $F(2,517)=28.94, p=0$. Multi comparison, $p_{c t r l}$, norm. $=0, p_{\text {ctrl, no-water }}=0.0001$, p porm., no-water $\left.=0.002\right)$.

We overserved a shift in BFs induced by normal sound exposure compared with the control group (Figure 4-2C), which was again a direct replication of previous results of the lab (Cruces-Solís et al., 2018). The shift in BFs was not towards the exposed frequency (16 $\mathrm{kHz}$ ) but towards higher frequencies. Besides, since here we recorded from a wider range of depths (Cruces-Solís et al. recorded from depths of 0-750 $\mu \mathrm{m}$ ), we could conclude the shift in BFs was homogeneous also at deeper sites, frequency-unspecific but might not extend throughout the inferior colliculus since it is not present at depths higher than 1000 ums. Similar pattern of BF shift was observed in no-water animals (Figure 4-2C), supporting our previous finding that water association was not the cause of plasticity induced by sound exposure in IC. Further quantification of changes in BF relative to the 
control across recording depths between 100 and $900 \mu \mathrm{m}$ confirmed our observation. The BF shift in no-water group was not as strong as the normal group, indicating water association may not be the cause of this shift but can strengthen it.

Cruces-Solis et al. showed that sound-exposure in the corner resulted in a local increase in spontaneous activity in the region adjacent to that tuned to the exposed frequency and a frequency-unspecific widening in bandwidth, without a change in response reliability (Cruces-Solís et al., 2018). To address whether we can replicate those changes in our study and also to further examine the effect of water association, we calculated response reliability, spontaneous activity and bandwidth. We divided recording sites based on their BFs into 3 regions: a "tuned" region with sites of $\mathrm{BF}$ around $16 \mathrm{kHz}(14.7-17.5 \mathrm{kHz})$, an "adjacent-" region with sites of BF tuned to 11.3-13.5 kHz and an "adjacent+" region with sites of $\mathrm{BF}$ tuned to $19-22.6 \mathrm{kHz}$ (Figure 4-3A). For each region, response reliability (defined as the percentage of trials had at least one spike in 0-80 ms window after stimulus onset) changed as a function of tone frequency in a similar way as the tuning curve that was high around tuned frequencies (Figure 4-3B). Consistent with previous results, increase in response gain was not accompanied by changes in peak response reliability (Figure 4-3C). However, spontaneous activity was higher for the normal exposure group in all regions and not only in the adjacent region as Cruces-Solis et al. (2018) found (Figure 4-3D). The inconsistence in the results cound have resulted from the fact that spontaneous activity in the inferior colliculus is rather variable (Figure 4-3D; spontaneous activity shows higher variability than other values). Our results show a frequency-unspecific increase in IC evoked and spontaneous activity induced by sound exposure. 
A

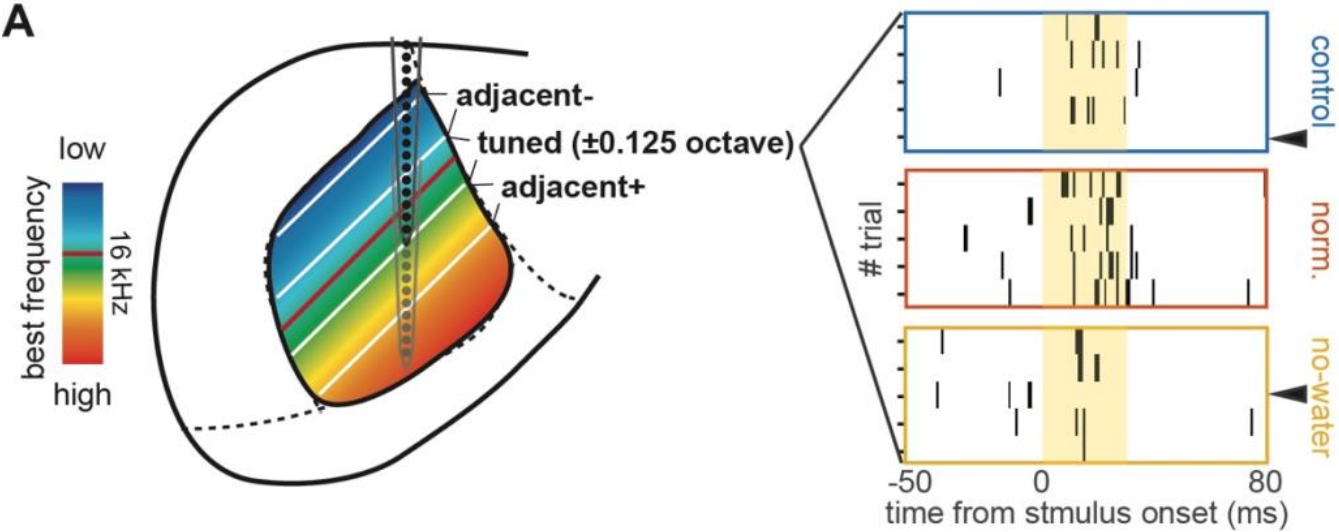

B
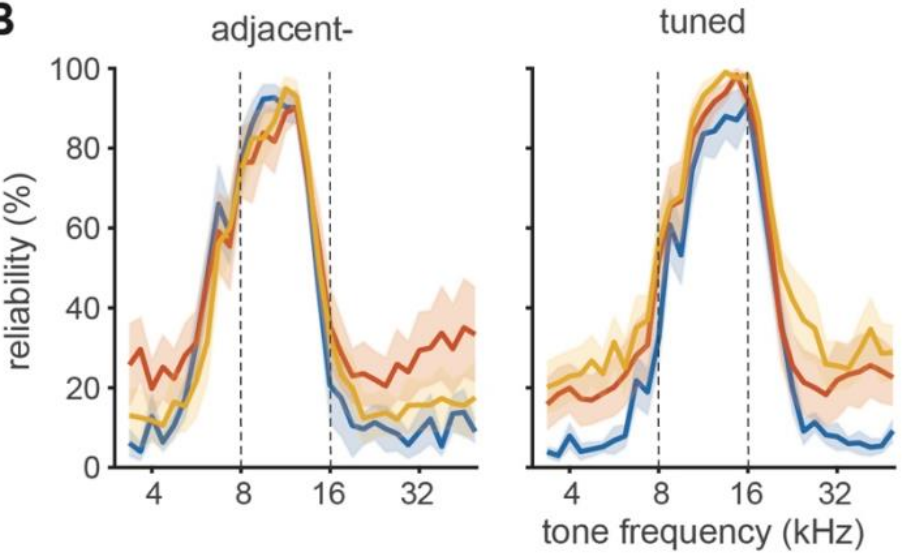

adjacent+

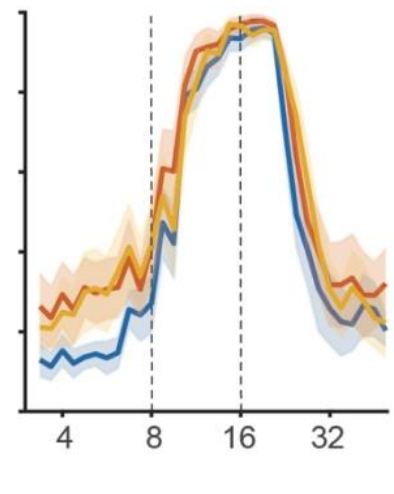

C

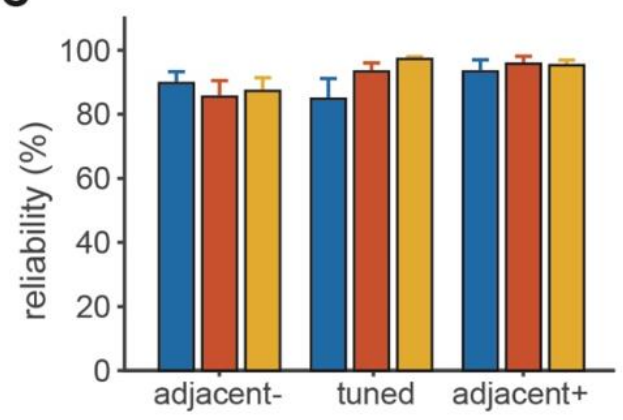

D

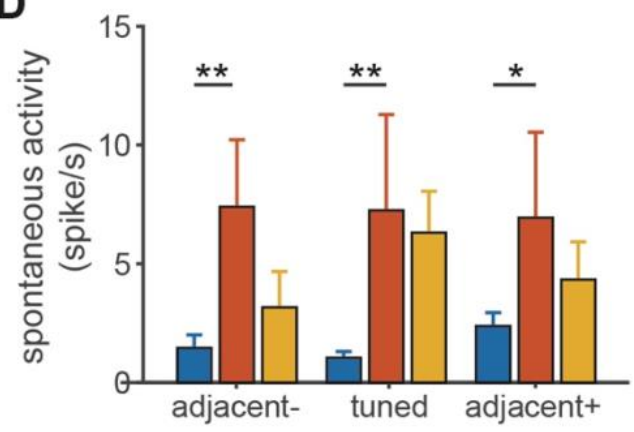

E

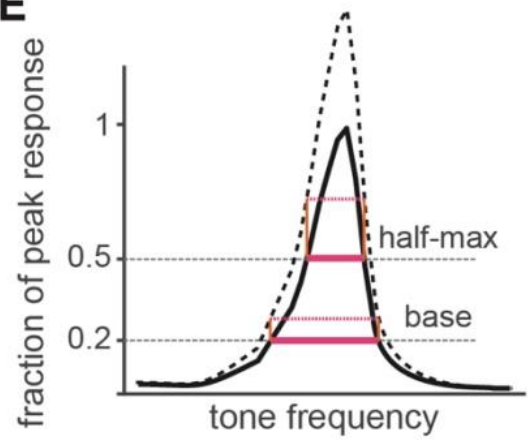

Figure 4-3 The effect of sound exposure was independent of water association

(A-left) Schematic representation of recording from dorsal adjacent (top, adjacent-), tuned (middle) and ventral adjacent (bottom, adjacent+) regions. (A-right) Example raster plots showing responses to $16 \mathrm{kHz}$ tone in the tuned region for each group. Sound stimuli are indicated by yellow bar. Trials without evoked spikes are indicated by black triangle. (B) Average response reliability as a function of tone frequency for each group in the adjacent (left, right) and tuned (middle) 
regions. (C) Average response reliability to the respective $\mathrm{BF} \pm 0.25$ octaves for adjacent and tuned regions (adjacent-, group $F(2,31)=0.23 p=0.8$; tuned, group $F(2,31)=2.92 p=0.07$; adjacent+, group $F(2,31)=0.24 p=0.79)$. (D) Average spontaneous activity for adjacent and tuned regions (adjacent- ANOVA, group $F(2,89)=6.05, p=0.004$; tuned ANOVA, group $F(2,152)=5.16, p=$ 0.007; adjacent+ ANOVA, group $F(2,212)=4.11, p=0.02$; multi comparison: ${ }^{*} \mathrm{p}<0.05,{ }^{* *} \mathrm{p}<$ $\left.0.01,{ }^{* * *} \mathrm{p}<0.001,{ }^{* * * *} \mathrm{p}<0.0001\right)$. For $(\mathbf{B}-\mathrm{D})$ number of recording sites: $n_{\text {ctrl, adjacent }}=24, n_{\text {ctrl, tuned }}=$ $46, n_{\text {ctrl, adjacent }}=51 ; n_{\text {norm., adjacent }}=33, n_{\text {norm., tuned }}=52, n_{\text {norm., adjacent }+}=88 ; n_{\text {ctrl, adjacent }}=35, n_{\text {ctrl, tuned }}=$ $57, n_{\mathrm{ctrl} \text {, adjacent }}=76$. (E-left) Schematic representation of base bandwidth and half-max bandwidth of the tuning curve (solid line). If the increase in response gain was homogenous across frequencies (dash line), there was no change in bandwidth. (E-middle) Average base bandwidth as a function of sound intensity (two-way ANOVA, group $F(2,1008)=2.43, p=0.09$, intensity $F(4,1008)=$ 86.2, $p=0$, interaction $F(8,1008)=0.85, p=0.56)$. (E-right) Same as $(\mathbf{E}$-middle) for half-max bandwidth (two-way ANOVA, group $F(2,1008)=11.09, p=0$, intensity $F(4,1008)=55.66, p=0$, interaction $F(8,1008)=0.49, p=0.86$; multi comparison, $p_{c t r l, \text { norm. }}=0, p_{c t r l, \text { no-water }}=0.0003$, pnorm., $_{\text {n }}$ no-water $=0.89)$.

If the increase in gain was homogenous throughout the tuning curve, we would expect to observe no change in bandwidth of tuning curve (Figure 4-3E left). However, both base and half-max bandwidth were decreased in the exposed groups (both normal and no-water group) compared with the control group (Figure 4-3E middle, right), and with a more robust change in half-max bandwidth (Figure 4-3E right). It suggests that the soundevoked responses to the tuned frequencies selectively increased more than the responses to the non-tuned frequencies. Therefore, increase in response gain was accompanied by an increase of the sharpness of tuning curve, reflecting an increase in neuronal discriminationability. This result was not consistent with the data from Cruces-Solís et al. where they found the bandwidhth increased in the normal exposure group with respect to control (Cruces-Solís et al., 2018). Removing the water from the corner led to the same changes in reliability, spontaneous activity or bandwidth, again suggesting that the presence of water in the corner did not play a role in plasticity but that rather the predictability fo the sound was the key parameter.

Overall, our results replicated the main findings previously reported in the lab on IC plasticity induced by sound exposure in the corner of the Audiobox. We confirmed that sound exposure elicited enhancement in overall response gain and a global shift in BF. This plasticity did not depend on the association between the sound exposure and having access to water. Besides, since no-water group had short exposure time than normal group (Figure 4-1E), the results also indicate that the duration of sound exposure did not influence the effect on IC plasticity. 


\subsubsection{Exposure to unpredictable sound in the corner does not elicit plasticity in the inferior colliculus}

We next examined the effect of exposure with unpredictable sound in the corner. Is the sound-context association alone enough for the generation of IC plasticity? We trained mice in the Audiobox and exposed them to a pure tone of $16 \mathrm{kHz}$ that was not, as before, strongly associated with the entry of the animals in the corner: the sound appeared only in $80 \%$ of the visits and never as the animal entered the corner but, instead, randomly 5-25 seconds after. Therefore, the timing of sound exposure could not be predicted by the animal's movement. Behaviorally, making the exposure sound unpredictable did not change the temporal pattern of the visit, reflected by no change in inter-visit-interval (Figure 4-1C) or average daily time spend in the corner (Figure 4-1D). The daily duration of sound exposure decreased (Figure 4-1E), since the sound was not played throughout each visit. Interestingly, the unpredictable group spent more time on drinking compared with other groups (Figure 4-1F).

Compared with the control group that was trained and recorded in parallel, the unpredictable group did not show changes in sound-evoked activity (Figure 4-4A, B; group $F(1,394)=0.66, p=0.42$ ). The average BFs were also comparable across depth (Figure 4-4C). Quantification of the mean difference in BF with respect to the control group across the depths between 100 and $900 \mu \mathrm{m}$ confirmed that there was no shift in BFs in the unpredictable group (Figure 4-4C inserted). Further analysis on response reliability (Figure 4-4D, E), spontaneous activity (Figure 4-4F) as well as tuning bandwidth (Figure 4-4G) revealed no significant change between the control and the unpredictable group. Therefore, unpredictable sound exposure and sound-context association did not elicit plasticity in IC. 

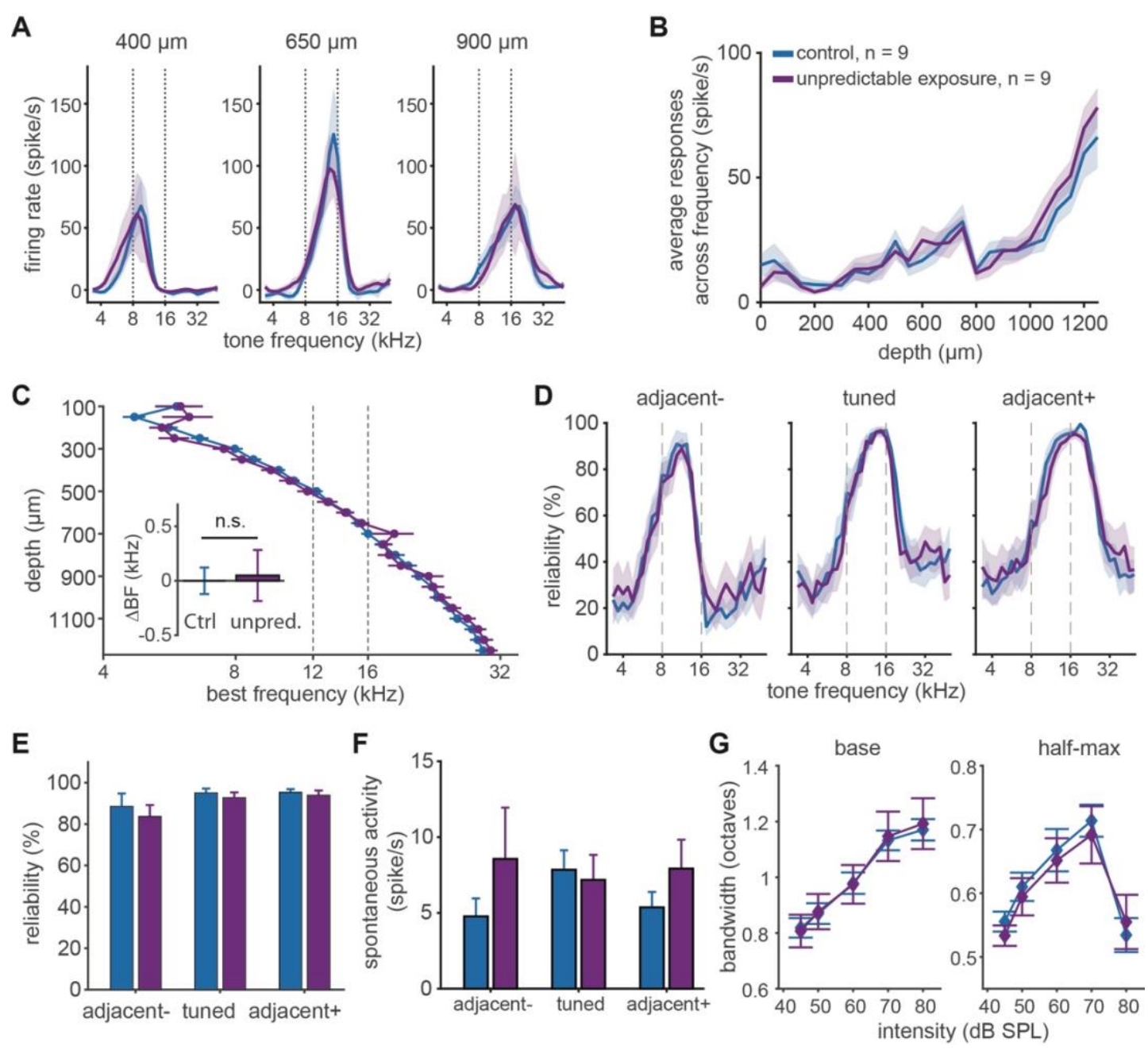

Figure 4-4 Unpredictable sound exposure did not elicit plasticity in the inferior colliculus

(A) Average tuning curves of sound-evoked activity at $70 \mathrm{~dB}$ for depth of 400,650 and $900 \mu \mathrm{m}$ in IC for control (blue) and unpredictable exposure (purple) group. (B) Average evoked responses across frequency at $70 \mathrm{~dB}$ as a function of recording depth (two-way ANOVA, group $F(1,394)=$ $0.66, p=0.42$, depth $F(25,394)=21.85, p=0$, interaction $F(25,394)=0.51, p=0.98) .(\mathbf{C})$ Average best frequency (BF) as a function of depths (two-way ANOVA, group $F(1,394)=1.39, p$ $=0.24$, depth $F(25,394)=32.4, p=0$, interaction $F(25,394)=0.78, p=0.77)$. Inserted figure: average difference in $\mathrm{BF}$ with respect to the average $\mathrm{BF}$ of control group across the depths between 100 and $900 \mu \mathrm{m}$ (unpaired t-test, $p=0.86$ ). (D) Average response reliability as a function of tone frequency for each group in the adjacent (left, right) and tuned (middle) regions. (E) Average response reliability to the respective $\mathrm{BF} \pm 0.25$ octaves for adjacent and tuned regions (unpaired ttest for each region: padjacent $=0.57$, ptuned $=0.50$, padjacent $+=0.62) .(\mathbf{F})$ Average spontaneous activity for adjacent and tuned regions (unpaired t-test for each region: padjacent $=0.17, p_{\text {tuned }}=0.87$, padjacent + $=0.30$ ). For $(\mathbf{D}-\mathbf{F})$ number of recording sites: $n_{\text {ctrl, adjacent }}=30, n_{\text {ctrl }, \text { tuned }}=51, n_{\text {ctrl, adjacent }}=43 ; n_{\text {unpred, }}$ adjacent $=26, n$ unpred., tuned $=47, n$ unpred., adjacent $=44$. $($ G-left $)$ Average base bandwidth as a function of sound intensity (two-way ANOVA, group $F(1,564)=0.01, p=0.92$, intensity $F(4,564)=16.77, p$ $=0$ ). (G-right) Same as (G-left) for half-max bandwidth (two-way ANOVA, group $F(1,564)=$ $0.65, p=0.42$, intensity $F(4,564)=16.91, p=0)$. 


\subsubsection{Sound exposure as a consequence of movement is the key factor for IC plasticity induced by sound exposure}

Comparing the normal exposure with no-water exposure revealed that the effect of sound-exposure on IC plasticity did neither depend on water association nor daily exposure time. Comparing the control group with the group with unpredictable sound exposure, ruled out that the plasticity resulted from sound-context association, further suggesting the plasticity in IC was caused by the association between sound and the animal's movement. To confirm this, we trained the mice in the Audiobox and exposed them with sound starting immediate after entering the corner but for only 3 seconds (entry-only group). Thus, the exposure sound could be predicted strongly by the animal's movement. Behaviorally, this sound exposure had no effect on the animals' behavior compared with normal exposure, reflected by no change in inter-visit-interval (Figure 4-1C), average daily time spend in the corner (Figure 4-1D) or percentage of drinking time (Figure 4-1F). The daily duration of sound exposure was, as expected, lower compared to other groups (Figure 4-1E).

Physiologically, the entry-only group show comparable sound-evoked activity as the normal exposure group that was trained and recorded in parallel (Figure 4-5A, B; group $F(1,394)=0.66, p=0.42)$. The average BFs were also not changed between groups (Figure 4-5C). Further analysis on response reliability (Figure 4-5D, E), spontaneous activity (Figure 4-5F) as well as tuning bandwidth (Figure 4-5G) revealed no significant change between the entry-only and the normal exposure group, with the exception that the spontaneous activity was higher in the adjacent+ region for the entry-only group. Taken together, our results indicate that sound exposure as a consequence of animal's movement was the key factor for changes observed in the inferior colliculus. 
A

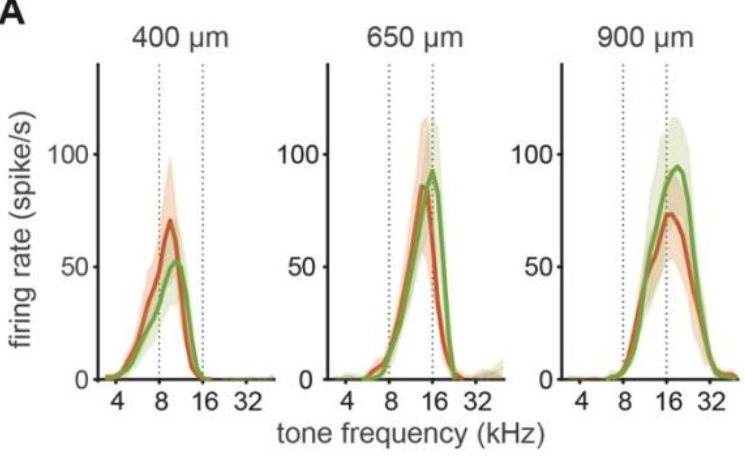

B

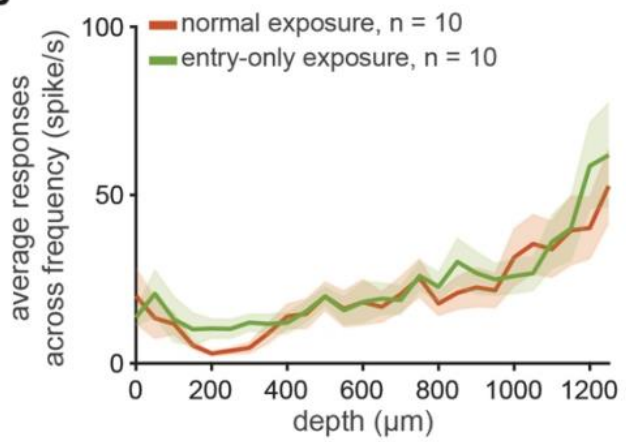

C

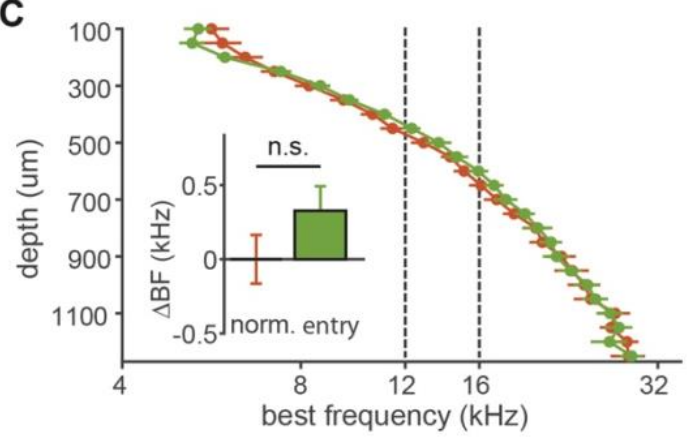

E

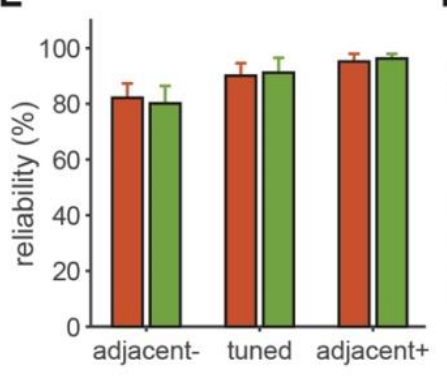

$\mathbf{F}$

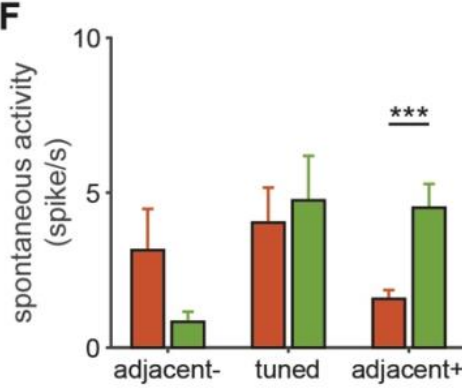

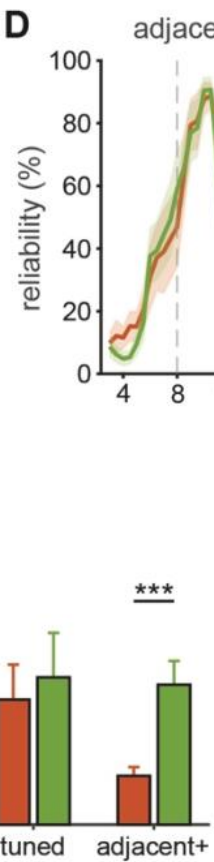

uned

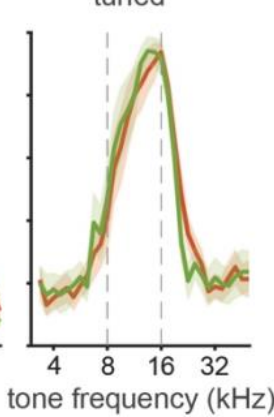

adjacent+
G

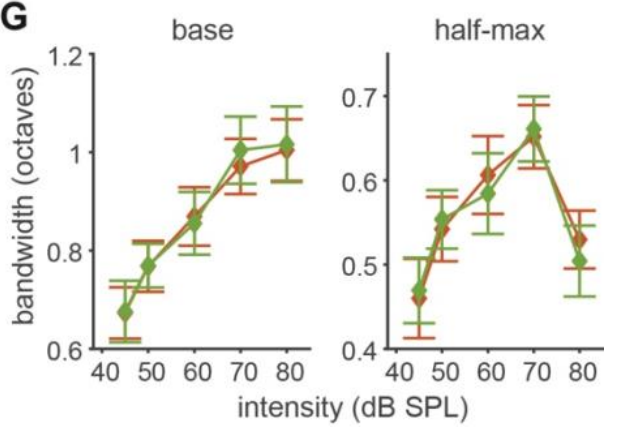

Figure 4-5 Movement-related sound exposure elicited plasticity in IC that was comparable to that of normal exposure

(A) Average tuning curves of sound-evoked activity at $70 \mathrm{~dB}$ for depth of 400,650 and $900 \mu \mathrm{m}$ in IC for normal (red) and entry-only (green) exposure group. (B) Average evoked responses across frequency at $70 \mathrm{~dB}$ as a function of recording depth (two-way ANOVA, group $F(1,447)=2.07, p$ $=0.15$, depth $F(25,447)=7.53, p=0$, interaction $F(25,448)=0.35, p=1) .(\mathbf{C})$ Average best frequency (BF) as a function of depths (two-way ANOVA, group $F(1,441)=1.3, p=0.25$, depth $F(25,441)=107.29, p=0$, interaction $F(25,441)=0.37, p=1)$. Inserted figure: average difference in $\mathrm{BF}$ with respect to the average $\mathrm{BF}$ of control group across the depths between 100 and $900 \mu \mathrm{m}$ (unpaired t-test, $p=0.16$ ). (D) Average response reliability as a function of tone frequency for each group in the adjacent (left, right) and tuned (middle) regions. (E) Average response reliability to the respective $\mathrm{BF} \pm 0.25$ octaves for adjacent and tuned regions (unpaired t-test for each region: padjacent $=0.81$, ptuned $=0.88$, padjacent $+=0.75)$. $(\mathbf{F})$ Average spontaneous activity for adjacent and tuned regions (unpaired t-test for each region: padjacent $_{-}=0.11, p_{\text {tuned }}=0.58$, padjacent ${ }^{+}=$ $0.0004)$. For (D-F) number of recording sites: $n_{\text {norm., adjacent }}=31, n_{\text {norm., tuned }}=58, n_{\text {norm., adjacent }}=54$; $n_{\text {entry, adjacent }}=27, n_{\text {entry, tuned }}=54, n_{\text {entry, adjacent }+}=65$. (G-left) Average base bandwidth as a function of sound intensity (two-way ANOVA, group $F(1,632)=1.25, p=0.26$, intensity $F(4,632)=40.64, p$ $=0$, interaction $F(4,632)=0.16, p=0.96)$. (G-right) Same as $(\mathbf{G}$-left $)$ for half-max bandwidth 
(two-way ANOVA, group $F(1,628)=0.35, p=0.56$, intensity $F(4,628)=19.97, p=0$, interaction $F(4,628)=0.53, p=0.71)$.

To sum up, we compared one of the main indexes that were affected by normal sound exposure, average BFs of each depth, for all tested groups (Figure 4-6A). Quantification of BF shift relative to control (Figure 4-6B) confirmed our finding that sound exposure could elicit global shift in BFs along the IC tonotopic axis only when the sound was a consequence of the animals' movement (predictable).
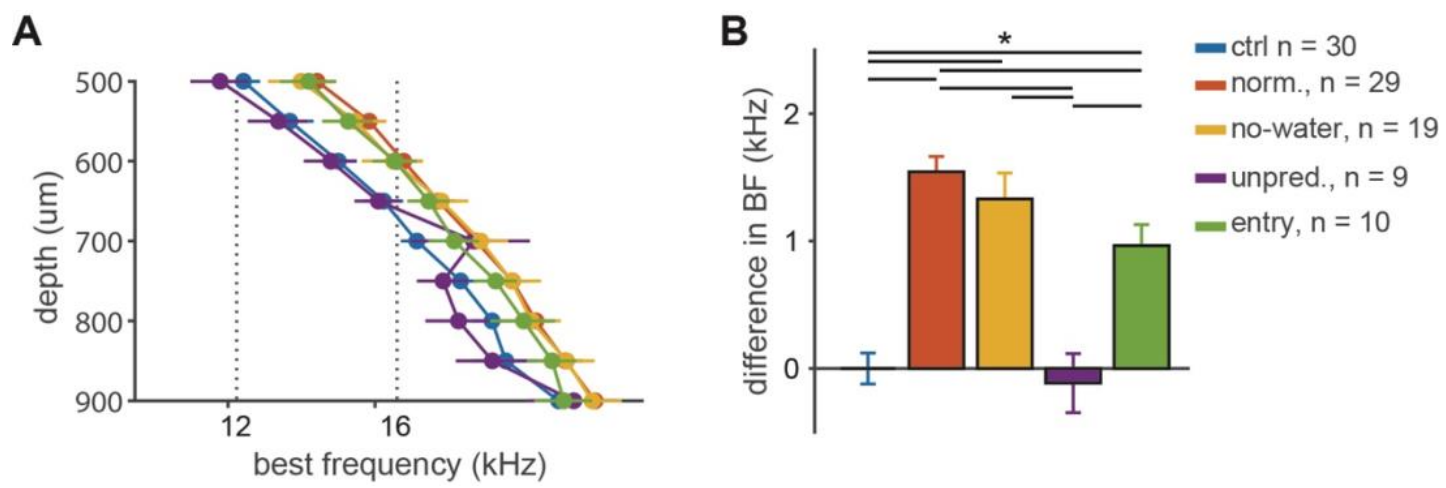

Figure 4-6 Predictable sound exposure elicited a global suprathreshold shift in BF

(A) Average best frequency (BF) as a function of depths (two-way ANOVA, group $F(4,1648)=$ $7.44, p=0$, depth $F(25,1648)=392.34, p=0$, interaction $F(100,1648)=0.8, p=0.93)$. (B) Average difference in BF with respect to the average BF of control group across the depths between 100 and $900 \mu \mathrm{m}$ (ANOVA, group $F(4,725)=26.29, p=0$, depth $F(16,725)=1.08, p=$ 0.37 , interaction $F(64,725)=0.46, p=1$. Multi comparison, ${ }^{*} p<0.05$.

Between replications, we found that the level of evoked activity, spontaneous activity, and tuning bandwidth varied. For the control group, the second replication (Figure 4-3 control vs. Figure 4-4 control) showed higher evoked and spontaneous activity, as well as wider bandwidth for sound intensity between 70-80 dB. For the normal exposure group, the second replication (Figure 4-3 norm. vs. Figure 4-5 norm.) showed lower evoked activity and spontaneous activity, as well as narrower bandwidth. The reason for this variation in replication is not clear. Animals within a replication and group were relatively consistent with each other. The variability could be caused by the variation between different batches of animals, differences in the recording probes which were reused within replications but not across. Thus, here we mainly did comparison between groups that were tested in parallel. However, as can be seen later, when averaging across replications the differences between groups held. 


\subsubsection{Sound exposure does not change sound-evoked or spontaneous activity in IC of freely behaving mouse}

To further test how the stimulus predictability affects neural activity in IC of the awake animal, we built a home-made shuttle box and designed a sound exposure paradigm with different degrees of predictability (Figure 0-6; see Method). The shuttle box has two compartments separated by an opening. The mouse initiated a predictable sound by crossing the division and entering the other side of the box. The predictable sound was played by a speaker close to the side being entered. As long as the animal was on one side, a random sound was played on that side every 10 to $40 \mathrm{~s}$. Thus, the mouse could not predict the stimulus onset. The predictable and random sounds were FMs with duration between 4 and 8 seconds of the same frequency range (either $5-20 \mathrm{kHz}$ or $6.5-26 \mathrm{kHz}$ ). For sounds played on the same side, the predictable and random sound had the same duration (either 20 or $80 \mathrm{~ms}$ ) but differed in sweep direction (Table $0-1$ ). We chose duration and sweep direction as two variables according to our behavioural findings (chapter 2) that discrimination of stimuli differing in those two dimensions was based on the perception of both dimensions. The four sounds used in our experiment, therefore, should not be automatically categorized based on their similarity on either sound feature. We implanted individually moveable tetrodes in the left inferior colliculus of naïve mice, like we did for the awake recordings described in chapter 3. Neural activity, including multiunit activity and local field potentials (LFPs), were recorded after recovery from surgery. For each daily session, the activity from the same set of units was recorded during the sound exposure (engaged) and during passive listening (passive) before and after the exposure (Figure 4-7A). During passive listening, pure tone pips with various frequency-intensity combinations were used to map the frequency-intensity tonal receptive field for each recording site. 


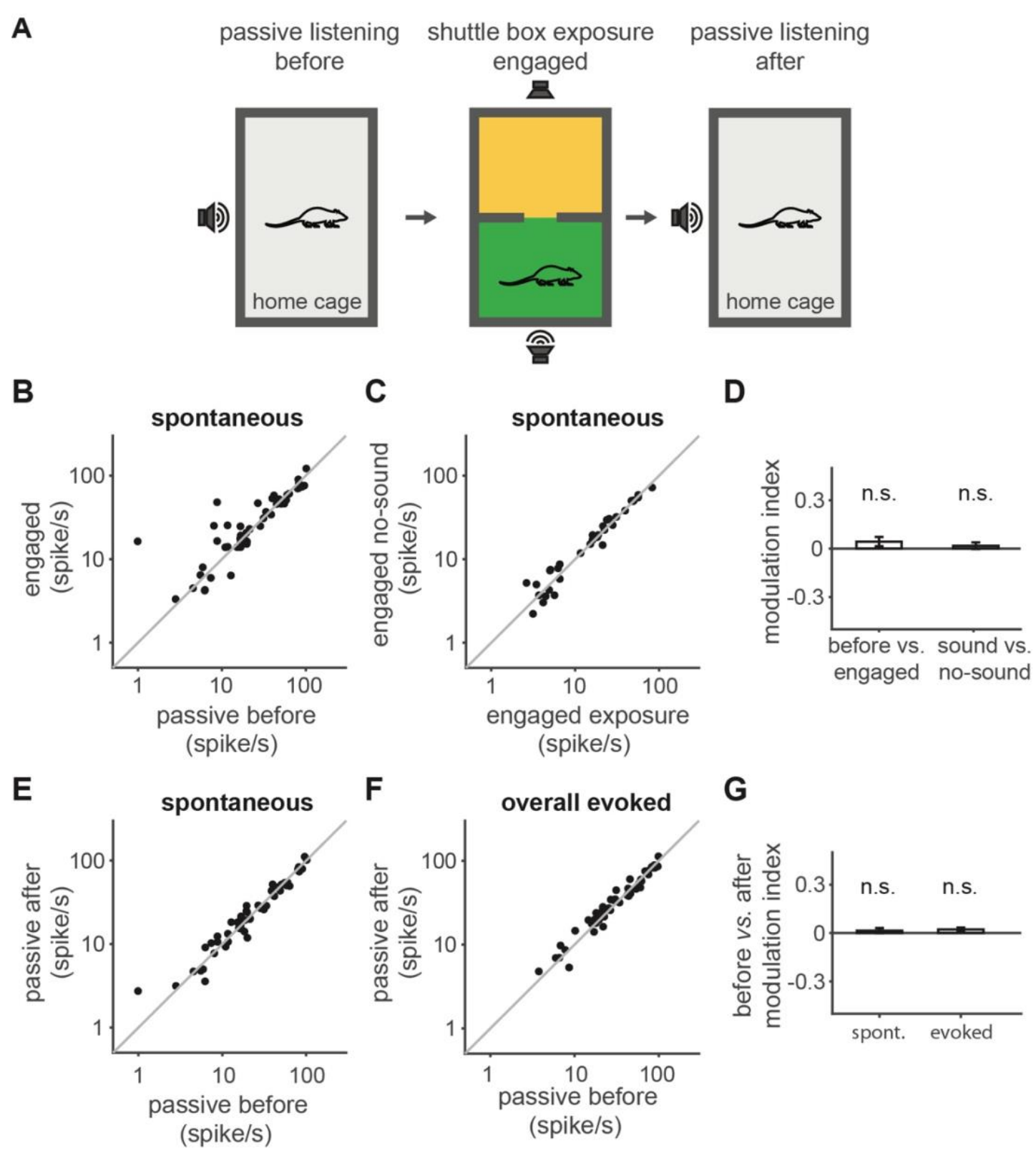

Figure 4-7 Sound exposure of FMs did not elicit change in response gain in IC of freely behaving mouse

(A) Schematic representation of daily recording paradigm. Mice had passive listening sessions immediately before and after sound exposure in the shuttle box. (B) Population spontaneous activity of multiunits comparing passive with engaged condition. (C) Same as (B) comparing during sound exposure with no-sound presentation in the shuttle box. (D) Modulation index for (B, C), respectively. (E) Same as (B) comparing passive condition before with after sound exposure. (F) Population overall sound-evoked activity of multiunits comparing passive before with after sound exposure. (G) Modulation index for $(\mathbf{E}, \mathbf{F})$, respectively. For (B-G), $n=52,3$ mice.

We first compared spontaneous activity when the mouse was in the shuttle box with activity when the mouse was passively listening (Figure 4-7B). Consistent with our findings that exposure to a safe sound in the sound terrace did not lead to changes in spontaneous activity, spontaneous activity was not changed by sound exposure in the 
shuttle box (Figure 4-7B, D-left; paired t-test, $p=0.28$ ). Considering the fact that sound presentation occurred only for some period when the mouse was in the shuttle box, we compared the spontaneous activity during sound exposure with activity when the mouse was in the shuttle box but not hearing any sound from a speaker. Again, there was no change in spontaneous activity in this comparison (Figure 4-7C, D-right; paired t-test, $p=$ $0.53)$.

We next examined whether there was a persistent change in neural activity immediately after sound exposure. We found that there was no change in either sound-evoked activity or spontaneous activity between the passive condition before and after the sound exposure session (Figure 4-7E-G; paired t-test, pevoed $=0.94$, p $_{\text {spont }}=0.47$ ).

To conclude, in freely behaving mouse, sound exposure in the shuttle box neither affected spontaneous activity, nor elicited persistent change in either sound-evoked or spontaneous activity in the period immediate after.

\subsubsection{Movement that initiates sound exposure suppresses ongoing local field potential in IC}

We further examined whether ongoing activity in IC was modulated by the crossing movement that was associated with predictable sound exposure in the shuttle box (Figure 4-8A). 

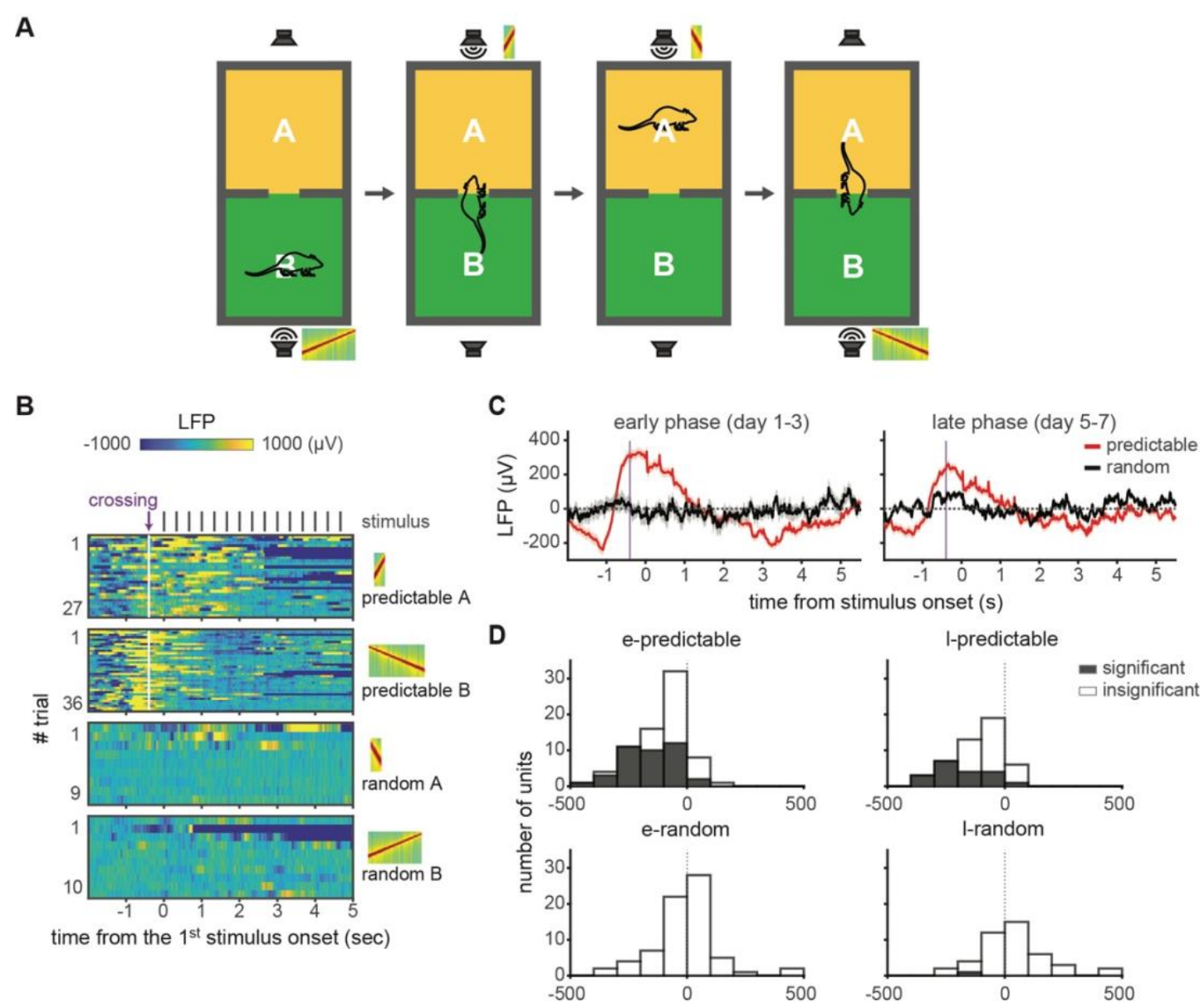

Figure 4-8 Self-initiation decreased ongoing local field potential in the inferior colliculus

(A) Schematic representation of sound exposure paradigm in the shuttle box. (B) Example LFP from one recording site during a sound exposure session. Trials were grouped based on the sound presented (spectrogram for each sound is shown on the right) and aligned to the 1 st stimulus onset (at 0 , the onset of each stimulus is shown as gray bar on the top). White line indicates the putative crossing movement which initiates the sound. (C) Average LFP during sound exposure of the predictable (red) and random (black) sounds during the early (left; $n=24,2$ mice) and late phase (right; $n=24,2$ mice). Trials were aligned to the $1_{\text {st }}$ stimulus onset. Vertical line indicates putative crossing movement. (D) Histogram plots the ongoing LFP change (0-1.2s vs. 1.5-4.2 sec) in each recording site for predictable (top panel) and random sounds (bottom panel) during the early (left panel) and late phase (right panel). Filled bars indicate sites showing significant changes $(p<0.05$, paired t-test; e-predictable $n=41 / 72,3$ mice; e-random $n=0 / 72,3$ mice; 1-predictable $n=19 / 48,2$ mice; 1-random $n=1 / 48,2$ mice).

We observed that during predictable, but not random, sound presentation the ongoing LFPs gradually became negative (Figure 4-8B, example LFPs from one site). The fluctuation in LFP was not caused by sound presentation, as it started prior to sound onset (Figure 4-8B, sound onset was at 0). The time course of this fluctuation was about 7 seconds. Starting at 2 seconds before sound onset, the LFP went down for about $1 \mathrm{sec}$, then went up and reached the positive peak when the mouse was crossing. After sound onset, the LFP gradually decreased, reached its negative peak after 3 seconds, and then gradually 
went back to its baseline. To investigate how this fluctuation developed with time, we analyzed LFPs separately for exposure day 1-3 (early phase) and day 5-7 (late phase). Average ongoing LFPs shows the same pattern of fluctuation in both early and late phase, but the magnitude of LFP change was weaker in the late phase (Figure 4-8C). Based on the time course of the LFP deflection, we quantified the modulation of LFPs by calculating the difference in mean LFP between the time window of 0-1.2 sec and 1.5-4.2 sec after sound onset. For both phases, most sites showed a negative change only during presentation of the predictable sound. In the early phase, mean LFP was changed during predictable sound exposure in $41 / 72$ (56.9\%; 3 mice, 3 days) recording sites, whereas during random sound exposure in $0 / 72(0 \% ; 3$ mice, 3 days) sites. In the late phase, the modulation was significant in 19/48 (39.6\%; 2 mice, 3 days) sites during predictable sound exposure, while $1 / 48$ (2.1\%; 2 mice, 3 days) during random sound exposure. Therefore, this confirmed our observation that the modulation effect only occurred during predictable sound presentation and was stronger in the early phase. The stronger modulation during the early phase may reflect a learning process to establish sound-movement association.

\subsection{Discussion}

Previous findings of the lab revealed that exposure to a sound that was not associated with either reward or punishment resulted in long-term plasticity in the inferior colliculus (Cruces-Solís et al., 2018). Plasticity in the inferior colliculus has been shown in bats and mice, but only for relative short time windows (Gao and Suga, 1998; Slee and David, 2015). Our results first show long-lasting plasticity can also happen in the auditory midbrain after context-sound association. In the experiment presented here, we further investigated the role of IC played on predictive information coding. The complexity of the environment in which animals are exposed makes the changes more naturalistic but at the same time require that we carefully disentangle the different contextual aspects that might have contributed to the predictability of the sound. To address this, we subjected mice to different sound exposure paradigms in which sounds were associated with different variables, such as water presence, context, or the animal's movement, in the same natural environment as before. We first replicated previous findings that sound exposure increased sound-evoked response grain and shifted the tonotopic map in the IC in a frequencyunspecific manner. Comparing the effect of different sound exposures paradigm, we found the plasticity was independent of water association or the duration of daily sound exposure, 
but strongly relied on the association between movement and the exposure sound that enabled prediction about sound onset. Furthermore, in freely behaving mice, the ongoing LFP fluctuated during the exposure to movement-initiated (predictable onset) sounds, but not random sounds that were presented independently of the animal's movement. However, we did not observe change in neither evoked nor spontaneous activity during sound exposure in freely moving animals. Together, our results demonstrate that the inferior colliculus is important in encoding the movement-sound association, which are of great importance in sensory filtering.

\subsubsection{Comparison with previous studies of the lab}

The sound-exposure induced plasticity observed in the IC largely replicated previous results of the lab with the same experimental setting (Cruces-Solís et al., 2018). We again observed a frequency-unspecific increase in response gain and BF shift. We could, therefore, confirm that the IC is involved in long-term learning of sparse contextassociated predictability. However, there were aspects of the results that differed form those published before, even though the experimental paradigm was replicated as exactly as possible. One such disagreement was reflected in the change in spontaneous activity. The global enhancement in sound-evoked response gain was accompanied with a global increase in spontaneous activity, while Cruces-Solís et al. found that the increase of spontaneous activity was specific for the region adjacent to that tuned to the exposure frequency $(16 \mathrm{kHz})$. It is worth noting that the variability of spontaneous activity was high in both studies, suggesting a inhomogeneous distribution of spontaneous activity in the IC. Indeed, others have shonw that trial-to-trial variability usually decreased when stimulus starts (He, 2013), indicating higher variability in spontaneous than evoked activity. Thus the difference between the two findings could have resulted from a difference in neuron population that was recorded. Another disagreement with previous findings from the lab was in the change in tuning bandwidth. In my hands, sound exposure decreased tuning bandwidth, particularly the half-max bandwidth, suggesting a sharpening of the tuning curve.

We recorded from a wider range of depths as in the previous study of the lab (0-125 um vs 0-750 ums), and found that the shift in BFs was limited to depths between 100 and 900 $\mu \mathrm{m}$. However, as described previously (Cruces-Solís et al., 2018), within this range the shift was homogeneous in size across depths, and always upwards. It remains to be 
investigated what is the benefit of this global BF shift. It has been reported frequently that associative learning, like classical conditioning, can cause stimulus-specific shift in the tonotopic map in the auditory system (Weinberger, 2004). Pairing tone and electric shock induced increase in response to the conditioned tone, while the response to the other frequencies decreased, resulting in a shift in the neuron's BF towards the conditioned frequency. However, the function of this type of plasticity in perception and learning is not yet well understood. The environment is complex, organisms need to constantly learn and update learnt information. If the plasticity is context-dependent, the organism can adapt quickly according to its current context (Carcea et al., 2017; Osmanski and Wang, 2015; Otazu et al., 2009). Long term plasticity results in memory traces in the cortex, but the direct link between this change in tonotopy and memory has not yet been established (Irvine, 2007). Further studies on understand the time window of how the plasticity that we observed in the IC developed and how long will it last when predictable sound exposure stopped will bring some hint to those questions.

We found changes in neither evoked nor spontaneous activity during or after sound exposure in freely behaving mice. Possible reasons for the discrepancies in results compared with anesthetized mice could be: 1) the plasticity observed in anesthetized mice was occurred after at least 6 days of sound exposure in the Audiobox (Cruces-Solís, 2016); 2) although movement-initiation was important in inducing plasticity, the effect of sound exposure could also be a result combination of multiple factors, like the enriched environment and movement association, which may not appeared in our awake experiment setting; 3) for freely behaving mice, FMs was used as exposure sound, which could evoke more neurons in the IC compared to pure tones of single frequency (Kuo and $\mathrm{Wu}, 2012$ ). Therefore, the change in tonotopy may not be visible; 4) Due to lack of direct comparison of evoked responses between engaged and passive conditions, it is not clear whether there was modulation of evoked activity during sound exposure;. Thus, we may not observe changes in IC within a daily session. Further recordings to track single neuron activity across days will help to characterize how plasticity in IC developed.

Whether and how these changes we observed influence perception and behavior is not yet well understood. Previous data from the lab showed that sound exposure led to a selective decrease in frequency discrimination acuity, a finding that was consistent with the increase in tuning bandwidth observed in the same study (Cruces-Solís et al., 2018). In my experiments, sound exposure did not change frequency discrimination acuity measured using startle reflex protocol (data not shown), which is in turn consistent with my finding 
that the tuning base bandwidth was not increased after exposure but rather decreased. In general, there are some inconsistences in the results compared with data from Cruces-Solís et al.. However, our data are very consistent within all the groups, like the level of activity, bandwidth, and BFs. Not enough is known about the patterns of plasticity in the IC. It is possible that we are recording from a slightly different region in the IC, for example in a more rostal column, that results in different pattern in plasticity. Further experiments to record from different areas of IC may shine light on how homogeneous the plasticity is in different subdivisions of IC.

\subsubsection{The movement-related plasticity in IC}

To our knowledge, our study is the first one to investigate whether associating a sound stimulus with self-movement elicits long-term plasticity in the auditory subcortical area, the inferior colliculus. It has been shown in numerous studies in both humans and a variety of animals that auditory responses to self-generated auditory event are attenuated compared to external sensations (Eliades and Wang, 2003; Phillips et al., 2017; Rummell et al., 2016; Sato, 2008). The suppression can not only be caused during vocalization (Eliades and Wang, 2003) but also during non-vocal behavior, including locomotion and whisking (Schneider et al., 2014). A more flexible form of movement-related suppression has also been found in the auditory cortex, i.e. associating movement with an temporalrelated external sound (expected) (Carcea et al., 2017; Rummell et al., 2016; Schneider et al., 2018). The mechanism of prediction has been suggested and confirmed by research in mice that shows the cortical responses to the "unexpected" movement-related sound are less attenuated (Eliades and Wang, 2008; Knolle et al., 2013; Rummell et al., 2016). Our finding that prediction-related plasticity in IC further indicates the specific attenuation of self-related sounds may also underlie long-lasting changes in the auditory system which may encode predictive information.

Our finding that the movement-sound association can lead to global increase in soundevoked activity seems to contradict the attenuation effect observed in the cortex during sound presentation. As the main ascending projections from the IC, the colliculogenicular projection is made of both glutamatergic and GABAergic projections (Ito et al., 2009; Ono et al., 2017). In rats, about $45 \%$ of neurons in the CNIC that projects to the MGB are GABAergic (Peruzzi et al., 1997). Those neurons have a unique morphology structure (larger in diameter) and have dense glutamatergic inputs that distinct from the other 
GABAergic neurons (Ito and Oliver, 2012; Ito et al., 2009; Ono et al., 2017). The functional role of those projections is not yet clear. One interpretation could be that the increase in activity in IC selectively affects these inhibitory projections to the thalamus, and thus result in decreased responses in the cortex. Indeed, it has been reported previously that predictable sound exposure induces changes in IC excitation/inhibition balance (Cruces-Solís et al., 2018). Another possibility could be that the increase in response can also further innervate stronger response in the thalamus. It has been found in awake rats, that engagement in an auditory task suppressed cortical evoked response, whereas the activity in the auditory thalamus was enhanced (Otazu et al., 2009).

Our results in freely behaving mice show a deflection in ongoing LFPs in the IC during presentation of predictable sounds. It seems to be consistent with previously mentioned studies in the cortex (Eliades and Wang, 2008; Knolle et al., 2013; Rummell et al., 2016), i.e. become negative during predictable sound presentation and the change starts prior to the onset of movement. However, we did not observe changes in ongoing spiking activity (data not shown). The LFPs can merge from multiple sources, including dendrite, soma, spine or axon (Buzsáki et al., 2012). Since we did not observe changes in spiking activity, the deflection of LFP may reflect a subthreshold excitatory-inhibitory imbalance (Friston et al., 2015).

So far, our data are still preliminary, we cannot completely rule out the possibility that the deflection in LFP was attributed to the movement, but not movement-related expectation. However, the stronger deflection observed in the early phase of the exposure, which cannot be explained by pure-movement mechanism, suggests this may related to learning of the movement-sound association.

To conclude, our result extends the role of IC in encoding movement-related auditory information, which to our knowledge has not been reported before. We found that to temporally associate sound stimuli as a consequence of self-movement was essential for the long-term plasticity in the IC induced by relevant sound exposure. Additionally, we found that movement that was associated with sound elicited changes in ongoing LFP of freely behaving animals prior to sound onset, supporting our finding in anesthetized animals that IC was sensitive to the movement-sound association. Movement-related modulation in the sensory system is often believed to have an filter effect caused by expectation of coming stimuli (Rummell et al., 2016; Schneider et al., 2018). However, researches are mainly focused in the sensory cortex, and these modulations are described 
as a short-term change. Here, we showed both short-term and long-term plasticity occurred in IC, thus placing IC at the center of processing of movement-related information. 


\section{CONCLUSIONS}

In this project, we aimed to better understand how sensation and memory interact to generate behavioral responses. On one hand, we studied animal's behavior during and after associative learning to infer the computation of memory processes; on the other hand, we investigated the role of the auditory midbrain, the IC, in associative learning and memory.

The first two chapters addressed the interaction between sensation and memory from a purely behavioral point of view. First, we questioned the influence of the physical properties of the stimuli on discrimination and generalization, with the aim to infer the constrain imposed by sensation on behavioral outcomes. We used a memory-based task to explore how the stimulus' physical properties, in this case tone frequency, affected auditory discrimination and generalization in mice. As expected, the physical distance between safe and conditioned sounds had a constraining effect on discrimination. As the safe-to-conditioned $\Delta F$ s decreased, performance deteriorated rapidly even though we used $\Delta$ Fs significantly larger than the reported frequency limens. Overall the data suggest that memory-based discriminations along a single stimulus dimension are inherently harder than those depended on relative-comparison and that there is a high overlap between the memory traces of the relevant stimuli. Given the relatively wide tuning in the auditory subcortical areas (Egorova et al., 2001; Taberner and Liberman, 2005), it is plausible that memory-based discriminations rely on wide sensory filters of subcortical regions. Then, we asked whether mice perceive sounds as a combination of features (frequency, direction of modulation, duration) or whether they can perceive the different stimulus features independently. We used a memory-based task to study the processing of multi-dimensional sounds. The idea behind this experiment was to infer the computation of complex sound processing through multidimensional generalization. We found that generalization of stimuli differing in two dimensions can be biased towards one dimension, such that variations in the other are ignored, or can be influenced by both dimensions. Whether one or both dimensions were generalized, depended on the combination of dimensions used during training. Certain sound features, like the sound frequency, were easier to learn and more likely to be generalized than other sound features, like sweep direction. This suggests a complex and maybe hierarchical processing pathway of multidimensional stimuli. Our 
result offers insights for future studies in choosing the optimal acoustic stimuli for both behavioral and physiological studies.

The inferior colliculus, based on its anatomical and physiological characteristics, seems a good candidate to be the first station where sensation interacts with memory. Early studies in head-fixed bats found that short-term plasticity occurred in the inferior colliculus after tone-shock pairing (Gao and Suga, 1998). Recently our lab found that long-term plasticity also happened in the IC of anesthetized mouse after context-associated sound exposure, in which sound was associated with neither reward nor punishment, supporting the view that IC is important in filtering auditory information. Our result extends the role of IC in encoding movement-related auditory information, which to our knowledge has not been reported before. We found that to temporally associate sound stimuli as a consequence of self-movement was essential for the long-term plasticity in the IC induced by sound exposure. Similar to previous data (Cruces-Solís et al., 2018), we observed increase in evoked and spontaneous activity in IC, as well as a stimuli-unspecific shift in BFs along the tonotopic axis. Additionally, we found that movement that was associated with sound, elicited changes in ongoing LFP of freely behaving animals prior to sound onset, supporting our finding in anesthetized animals that IC was sensitive to movementsound association. Movement-related modulation in sensory system is often believed to be an filter effect caused by expectation of coming stimuli (Rummell et al., 2016; Schneider et al., 2018). But researches are mainly centred in the sensory cortex, and these modulations are described as a short-term change. Here, we showed both short-term and long-term plasticity occurred in IC, placing IC at the centre of processing of movement-related information. Besides, we found neural activity in IC of freely behaving mice can also be modulated during reinforcement learning task in which sound stimuli was associated with either reward or punishment. Sound-evoked response to rewarded-stimulus was overall not changed, while evoked responses to the conditioned stimuli were suppressed. The suppression in response to the target sound is consistent with previous findings in headfixed ferrets during performance in an auditory detection task (Slee and David, 2015). Modulation of baseline and evoked activity by task engagement has been thoroughly demonstrated in the cortex. Our finding that similar patterns can be observed in the auditory midbrain suggests that subcortical structures play a more important role in associative learning than previously thought. Together with the result that we found increase in spontaneous activity in the IC of exposed, not conditioned, animals. Regulation 
of spontaneous activity seems to be a common mechanism underlying sensory gating in the IC. The IC is, therefore, sensitive to the relevance of sounds and to task-performance.

To sum up, both behavioral and physiological results point to the same conclusion that auditory subcortical structures is important in interpreting sound meaning, and thus play a crucial role in the interaction between sensation and memory. 


\section{REFERENCES}

Ahissar, M., and Hochstein, S. (1997). Task difficulty and the specificity of perceptual learning. Nature 387, 401-406.

Ahissar, M., Nahum, M., Nelken, I., and Hochstein, S. (2009). Reverse hierarchies and sensory learning. Philos. Trans. R. Soc. B Biol. Sci. 364, 285-299.

Ahrens, S., Jaramillo, S., Yu, K., Ghosh, S., Hwang, G.R., Paik, R., Lai, C., He, M., Huang, Z.J., and Li, B. (2015). ErbB4 regulation of a thalamic reticular nucleus circuit for sensory selection. Nat. Neurosci. 18, 104-111.

Åhs, F., Miller, S.S., Gordon, A.R., and Lundström, J.N. (2013). Aversive learning increases sensory detection sensitivity. Biol. Psychol. 92, 135-141.

Aizenberg, M., and Neimark Geffen, M. (2013). Bidirectional effects of aversive learning on perceptual acuity are mediated by the sensory cortex. Nat. Neurosci. 16, 994996.

Aluisi, F., Rubinchik, A., and Morris, G. (2018). Animal Learning in a Multidimensional Discrimination Task as Explained by Dimension-Specific Allocation of Attention Flavia. 12, 356.

Amitay, S., Irwin, A., and Moore, D.R. (2006). Discrimination learning induced by training with identical stimuli. Nat. Neurosci. 9, 1446-1448.

Ashby, F.G., and Rosedahl, L. (2017). A neural interpretation of exemplar theory. Psychol. Rev. 124, 472-482.

Atencio, C.A., and Schreiner, C.E. (2010). Laminar Diversity of Dynamic Sound Processing in Cat Primary Auditory Cortex. J. Neurophysiol. 103, 192-205.

Atiani, S., Elhilali, M., David, S. V., Fritz, J.B., and Shamma, S.A. (2009). Task difficulty and performance induce diverse adaptive patterns in gain and shape of primary auditory cortical receptive fields. Neuron $61,467-480$.

Ayala, Y.A., and Malmierca, M.S. (2015). Cholinergic Modulation of StimulusSpecific Adaptation in the Inferior Colliculus. J. Neurosci. 35, 12261-12272.

Babaev, O., Botta, P., Meyer, E., Müller, C., Ehrenreich, H., Brose, N., Lüthi, A., and Krueger-Burg, D. (2016). Neuroligin 2 deletion alters inhibitory synapse function and anxiety-associated neuronal activation in the amygdala. Neuropharmacology 100, 56-65.

Bagur, S., Averseng, M., Elgueda, D., David, S., Fritz, J., Yin, P., Shamma, S., Boubenec, Y., and Ostojic, S. (2018). Go/No-Go task engagement enhances population representation of target stimuli in primary auditory cortex. Nat. Commun. 9, 2529. 
Bakin, J.S., and Weinberger, N.M. (1990). Classical conditioning induces CS-specific receptive field plasticity in the auditory cortex of the guinea pig. Brain Res. 536, 271-286.

Bang, S.J., Allen, T.A., Jones, L.K., Boguszewski, P., and Brown, T.H. (2008). Asymmetrical stimulus generalization following differential fear conditioning. Neurobiol. Learn. Mem. 90, 200-216.

Barberini, C.L., Morrison, S.E., Saez, A., Lau, B., and Salzman, C.D. (2012). Complexity and competition in appetitive and aversive neural circuits. Front. Neurosci. 6 , 170.

Barnstedt, O., Keating, P., Weissenberger, Y., King, A.J., and Dahmen, J.C. (2015). Functional Microarchitecture of the Mouse Dorsal Inferior Colliculus Revealed through In Vivo Two-Photon Calcium Imaging. J. Neurosci. 35, 10927-10939.

Barton, B., Venezia, J.H., Saberi, K., Hickok, G., and Brewer, A.A. (2012). Orthogonal acoustic dimensions define auditory field maps in human cortex. Proc. Natl. Acad. Sci. U. S. A. $109,20738-20743$.

Basavaraj, S., and Yan, J. (2012). Prepulse Inhibition of Acoustic Startle Reflex as a Function of the Frequency Difference between Prepulse and Background Sounds in Mice. PLoS One 7, e45123.

Beaton, R., and Miller, J.M. (1975). Single cell activity in the auditory cortex of the unanesthetized, behaving monkey: correlation with stimulus controlled behavior. Brain Res. 100, 543-562.

Beitel, R.E., Schreiner, C.E., Cheung, S.W., Wang, X., and Merzenich, M.M. (2003). Reward-dependent plasticity in the primary auditory cortex of adult monkeys trained to discriminate temporally modulated signals. Proc. Natl. Acad. Sci. 100, 11070-11075.

Bennett, C., Arroyo, S., and Hestrin, S. (2013). Subthreshold mechanisms underlying state-dependent modulation of visual responses. Neuron 80, 350-357.

Beyeler, A., Namburi, P., Glober, G.F., Simonnet, C., Calhoon, G.G., Conyers, G.F., Luck, R., Wildes, C.P., and Tye, K.M. (2016). Divergent Routing of Positive and Negative Information from the Amygdala during Memory Retrieval. Neuron 90, 348-361.

Bieszczad, K.M., and Weinberger, N.M. (2010). Representational gain in cortical area underlies increase of memory strength. Proc. Natl. Acad. Sci. 107, 3793-3798.

Bitterman, Y., Mukamel, R., Malach, R., Fried, I., and Nelken, I. (2008). Ultra-fine frequency tuning revealed in single neurons of human auditory cortex. Nature 451, 197201.

Blakemore, S.J., Frith, C.D., and Wolpert, D.M. (1999). Spatio-temporal prediction 
modulates the perception of self-produced stimuli. J. Cogn. Neurosci. 11, 551-559.

Blough, D.S. (1969). Generalization gradient shape and summation in steady-state tests. J. Exp. Anal. Behav. 12, 91-104.

Blundell, J., Tabuchi, K., Bolliger, M.F., Blaiss, C.A., Brose, N., Liu, X., Südhof, T.C., and Powell, C.M. (2009). Increased anxiety-like behavior in mice lacking the inhibitory synapse cell adhesion molecule neuroligin 2. Genes, Brain Behav. 8, 114-126.

Bouton, M.E., and Moody, E.W. (2004). Memory processes in classical conditioning. Neurosci. Biobehav. Rev. 28, 663-674.

Brand, A., Urban, A., and Grothe, B. (2000). Duration Tuning in the Mouse Auditory Midbrain.

Brewer, A.A., and Barton, B. (2016). Maps of the Auditory Cortex. Annu. Rev. Neurosci. 39, 385-407.

Brosch, M., Selezneva, E., and Scheich, H. (2011). Representation of reward feedback in primate auditory cortex. Front. Syst. Neurosci. 5, 5.

Brosch, M., Selezneva, E., and Scheich, H. (2015). Neuronal activity in primate auditory cortex during the performance of audiovisual tasks. Eur. J. Neurosci. 41, 603-614.

Brugge, J.F. (1992). An Overview of Central Auditory Processing. (Springer, New York, NY), pp. 1-33.

Buzsáki, G., Anastassiou, C.A., and Koch, C. (2012). The origin of extracellular fields and currents--EEG, ECoG, LFP and spikes. Nat. Rev. Neurosci. 13, 407-420.

Caporale, N., and Dan, Y. (2008). Spike Timing-Dependent Plasticity: A Hebbian Learning Rule. Annu. Rev. Neurosci. 31, 25-46.

Carcea, I., Insanally, M.N., Froemke, R.C., Carcea, A.I., Insanally, M.N., and Froemke, R.C. (2017). Dynamics of cortical activity during behavioral engagement and auditory perception. Nat. Commun. 8, 1-12.

Casseday, J.H.H., Fremouw, T., and Covey, E. (2002). The Inferior Colliculus: A Hub for the Central Auditory System. (Springer, New York, NY), pp. 238-318.

Chambers, C., Akram, S., Adam, V., Pelofi, C., Sahani, M., Shamma, S., and Pressnitzer, D. (2017). Prior context in audition informs binding and shapes simple features. Nat. Commun. 8, 15027.

Chavez, C.M., McGaugh, J.L., and Weinberger, N.M. (2013). Activation of the basolateral amygdala induces long-term enhancement of specific memory representations in the cerebral cortex. Neurobiol. Learn. Mem. 101, 8-18.

Chechik, G., and Nelken, I. (2012). Auditory abstraction from spectro-temporal 
features to coding auditory entities. Proc. Natl. Acad. Sci. U. S. A. 109, 18968-18973.

Chechik, G., Anderson, M.J., Bar-Yosef, O., Young, E.D., Tishby, N., and Nelken, I. (2006). Reduction of Information Redundancy in the Ascending Auditory Pathway. Neuron 51, 359-368.

Chen, M., and Bargh, J.A. (1999). Consequences of Automatic Evaluation: Immediate Behavioral Predispositions to Approach or Avoid the Stimulus. Personal. Soc. Psychol. Bull. 25, 215-224.

Chen, C.-H., Lee, P.-W., Liao, H.-M., and Chang, P.-K. (2017). Neuroligin 2 R215H Mutant Mice Manifest Anxiety, Increased Prepulse Inhibition, and Impaired Spatial Learning and Memory. Front. Psychiatry 8, 257.

Clause, A., Nguyen, T., and Kandler, K. (2011). An acoustic startle-based method of assessing frequency discrimination in mice. J. Neurosci. Methods 200, 63-67.

Claverol-Tinture, E., and Nadasdy, Z. (2004). Intersection of Microwire Electrodes With Proximal CA1 Stratum-Pyramidale Neurons at Insertion for Multiunit Recordings Predicted by a 3-D Computer Model. IEEE Trans. Biomed. Eng. 51, 2211-2216.

Cruces-Solís, H. (2016). Neuronal correlates of implicit learning in the mammalian midbrain Hugo Cruces Solís.

Cruces-Solís, H., Jing, Z., Babaev, O., Rubin, J., Gür, B., Krueger-Burg, D., Strenzke, N., and de Hoz, L. (2018). Auditory midbrain coding of statistical learning that results from discontinuous sensory stimulation. PLOS Biol. 16, e2005114.

Cruikshank, S.J., Edeline, J.M., and Weinberger, N.M. (1992). Stimulation at a site of auditory-somatosensory convergence in the medial geniculate nucleus is an effective unconditioned stimulus for fear conditioning. Behav. Neurosci. 106, 471-483.

Dadarlat, M.C., and Stryker, M.P. (2017). Locomotion Enhances Neural Encoding of Visual Stimuli in Mouse V1. J. Neurosci. 37, 3764-3775.

David, S. V., Fritz, J.B., and Shamma, S.A. (2012). Task reward structure shapes rapid receptive field plasticity in auditory cortex. Proc. Natl. Acad. Sci. 109, 2144-2149.

Davis, M. (1992). The Role of the Amygdala in Fear and Anxiety. Annu. Rev. Neurosci. 15, 353-375.

Delhommeau, K., Micheyl, C., Jouvent, R., and Collet, L. (2002). Transfer of learning across durations and ears in auditory frequency discrimination. Percept. Psychophys. 64, 426-436.

Dibbets, P., and Evers, E.A.T. (2017). The Influence of State Anxiety on Fear Discrimination and Extinction in Females. Front. Psychol. 08, 347. 
Dipoppa, M., Ranson, A., Krumin, M., Pachitariu, M., Carandini, M., and Harris, K.D. (2018). Vision and Locomotion Shape the Interactions between Neuron Types in Mouse Visual Cortex. Neuron 98, 602-615.e8.

Doucet, J.R.R., Molavi, D.L.L., and Ryugo, D.K.K. (2003). The source of corticocollicular and corticobulbar projections in area Te1 of the rat. Exp. Brain Res. 153, 461-466.

Dunsmoor, J.E., and Paz, R. (2015). Fear Generalization and Anxiety: Behavioral and Neural Mechanisms. Biol. Psychiatry 78, 336-343.

Duque, D., and Malmierca, M.S. (2015). Stimulus-specific adaptation in the inferior colliculus of the mouse: anesthesia and spontaneous activity effects. Brain Struct. Funct. 220, 3385-3398.

Edeline, J.-M., and Weinberger, N.M. (1991). Thalamic short-term plasticity in the auditory system: Associative retuning of receptive fields in the ventral medial geniculate body. Behav. Neurosci. 105, 618-639.

Edeline, J.-M., and Weinberger, N.M. (1993). Receptive field plasticity in the auditory cortex during frequency discrimination training: Selective retuning independent of task difficulty. Behav. Neurosci. 107, 82-103.

Edeline, J.M., Dutrieux, G., Manunta, Y., and Hennevin, E. (2001). Diversity of receptive field changes in auditory cortex during natural sleep. Eur. J. Neurosci. 14, 18651880 .

Egorova, M., Vartanyan, I., and Ehret, G. (2006). Frequency response areas of mouse inferior colliculus neurons: II. Critical bands. Neuroreport 17, 1783-1786.

Ehret, G. (1975). Frequency and intensity difference limens and nonlinearities in the ear of the housemouse (Mus musculus). J. Comp. Physiol. ? A 102, 321-336.

Ehret, G. (1976). Critical bands and filter characteristics in the ear of the housemouse (Mus musculus). Biol. Cybern. 24, 35-42.

Ehret, G. (1992). Categorical perception of mouse-pup ultrasounds in the temporal domain. Anim. Behav. 43, 409-416.

Ehret, G., and Merzenich, M.M. Complex sound analysis (frequency resolution, filtering and spectral integration) by single units of the inferior colliculus of the cat. Brain Res. 472, 139-163.

Ehret, G., and Merzenich, M.M. (1985). Auditory midbrain responses parallel spectral integration phenomena. Science 227, 1245-1247.

Ehret, G., and Riecke, S. (2002). Mice and humans perceive multiharmonic 
communication sounds in the same way. Proc. Natl. Acad. Sci. U. S. A. 99, 479-482.

Eliades, S.J., and Wang, X. (2003). Sensory-Motor Interaction in the Primate Auditory Cortex During Self-Initiated Vocalizations. J. Neurophysiol. 89, 2194-2207.

Eliades, S.J., and Wang, X. (2008). Neural substrates of vocalization feedback monitoring in primate auditory cortex. Nature 453, 1102-1106.

Engineer, N.D., Percaccio, C.R., Pandya, P.K., Moucha, R., Rathbun, D.L., and Kilgard, M.P. (2004). Environmental Enrichment Improves Response Strength, Threshold, Selectivity, and Latency of Auditory Cortex Neurons. J. Neurophysiol. 92, 73-82.

Fay, R.R. (1974). Auditory frequency discrimination in vertebrates. J. Acoust. Soc. Am. 56, 206-209.

Ferezou, I., and Deneux, T. (2017). Review: How do spontaneous and sensory-evoked activities interact? Neurophotonics 4, 031221.

Fields, H.L., Hjelmstad, G.O., Margolis, E.B., and Nicola, S.M. (2007). Ventral Tegmental Area Neurons in Learned Appetitive Behavior and Positive Reinforcement. Annu. Rev. Neurosci. 30, 289-316.

Florentine, M. (1983). Intensity discrimination as a function of level and frequency and its relation to high-frequency hearing. J. Acoust. Soc. Am. 74, 1375-1379.

Friston, K.J., Bastos, A.M., Pinotsis, D., and Litvak, V. (2015). LFP and oscillationswhat do they tell us? Curr. Opin. Neurobiol. 31, 1-6.

Fritz, J., Shamma, S., Elhilali, M., and Klein, D. (2003). Rapid task-related plasticity of spectrotemporal receptive fields in primary auditory cortex. Nat. Neurosci. 6, 1216-1223.

Fritz, J.B., Elhilali, M., and Shamma, S.A. (2005). Differential Dynamic Plasticity of A1 Receptive Fields during Multiple Spectral Tasks. J. Neurosci. 25, 7623-7635.

Fritz, J.B., Elhilali, M., David, S. V., and Shamma, S.A. (2007). Does attention play a role in dynamic receptive field adaptation to changing acoustic salience in A1? Hear. Res. 229, 186-203.

Gaese, B.H., King, I., Felsheim, C., Ostwald, J., and von der Behrens, W. (2006). Discrimination of direction in fast frequency-modulated tones by rats. J. Assoc. Res. Otolaryngol. 7, 48-58.

Galambos, R., Sheatz, G., and Vernier, V.G. (1956). Electrophysiological correlates of a conditioned response in cats. Science (80-. ). 123, 376-377.

Gao, E., and Suga, N. (1998). Experience-dependent corticofugal adjustment of midbrain frequency map in bat auditory system. Proc. Natl. Acad. Sci. U. S. A. 95, 1266312670. 
Gao, E., and Suga, N. (2000). Experience-dependent plasticity in the auditory cortex and the inferior colliculus of bats: role of the corticofugal system. Proc. Natl. Acad. Sci. U. S. A. $97,8081-8086$.

Geissler, D.B., and Ehret, G. (2002). Time-critical integration of formants for perception of communication calls in mice. Proc. Natl. Acad. Sci. U. S. A. 99, 9021-9025.

Ghirlanda, S., and Enquist, M. (2003). A century of generalization. Anim. Behav. 66, $15-36$.

Gittelman, J.X., Perkel, D.J., and Portfors, C. V. (2013). Dopamine Modulates Auditory Responses in the Inferior Colliculus in a Heterogeneous Manner. J. Assoc. Res. Otolaryngol. 14, 719-729.

Grill-Spector, K., and Malach, R. (2004). THE HUMAN VISUAL CORTEX. Annu. Rev. Neurosci. 27, 649-677.

Grimault, N., Micheyl, C., Carlyon, R.P., Bacon, S.P., and Collet, L. (2003). Learning in discrimination of frequency or modulation rate: generalization to fundamental frequency discrimination. Hear. Res. 184, 41-50.

Gruters, K.G., and Groh, J.M. (2012). Sounds and beyond: multisensory and other nonauditory signals in the inferior colliculus. Front. Neural Circuits 6, 96.

Hage, S.R., and Ehret, G. (2003). Mapping responses to frequency sweeps and tones in the inferior colliculus of house mice. Eur. J. Neurosci. 18, 2301-2312.

Hage, S.R., Nter Ehret, G.È., and Ehret, G.G. (2003). Mapping responses to frequency sweeps and tones in the inferior colliculus of house mice. Eur. J. Neurosci. 18, 2301-2312.

Haider, B., Duque, A., Hasenstaub, A.R., Yu, Y., and McCormick, D.A. (2007). Enhancement of visual responsiveness by spontaneous local network activity in vivo. J. Neurophysiol. 97, 4186-4202.

He, B.J. (2013). Spontaneous and task-evoked brain activity negatively interact. J. Neurosci. 33, 4672-4682.

Heffner, H., and Masterton, B. (1980). Hearing in Glires: Domestic rabbit, cotton rat, feral house mouse, and kangaroo rat. J. Acoust. Soc. Am. 68, 1584-1599.

Hegdé, J. (2008). Time course of visual perception: Coarse-to-fine processing and beyond. Prog. Neurobiol. 84, 405-439.

Heil, P., Langner, G., and Scheich, H. (1992). Processing of frequency-modulated stimuli in the chick auditory cortex analogue: evidence for topographic representations and possible mechanisms of rate and directional sensitivity. J Comp Physiol A 171, 583-600.

Hensch, T.K. (2005). Critical period plasticity in local cortical circuits. Nat. Rev. 
Neurosci. 6, 877-888.

Herbert, H., Klepper, A., and Ostwald, J. (1997). Afferent and efferent connections of the ventrolateral tegmental area in the rat. Anat. Embryol. (Berl). 196, 235-259.

Hochstein, S., and Ahissar, M. (2002). View from the top: Hierarchies and reverse hierarchies in the visual system. Neuron 36, 791-804.

de Hoz, L., and Nelken, I. (2014). Frequency Tuning in the Behaving Mouse: Different Bandwidths for Discrimination and Generalization. PLoS One 9, e91676.

Hu, H. (2016). Reward and Aversion. Annu. Rev. Neurosci. 39, 297-324.

Irvine, D.R.F. (2007). Auditory cortical plasticity: does it provide evidence for cognitive processing in the auditory cortex? Hear. Res. 229, 158-170.

Irvine, D.R.F. (2018a). Plasticity in the auditory system. Hear. Res. 362, 61-73.

Irvine, D.R.F. (2018b). Auditory perceptual learning and changes in the conceptualization of auditory cortex. Hear. Res. 366, 3-16.

Ito, T., and Oliver, D.L. (2012). The basic circuit of the IC: tectothalamic neurons with different patterns of synaptic organization send different messages to the thalamus. Front Neural Circuits 6, 48.

Ito, T., Bishop, D.C., and Oliver, D.L. (2009). Two Classes of GABAergic Neurons in the Inferior Colliculus. J. Neurosci. 29.

Janak, P.H., and Tye, K.M. (2015). From circuits to behaviour in the amygdala. Nature 517, 284-292.

Jaramillo, S., and Zador, A.M. (2011). The auditory cortex mediates the perceptual effects of acoustic temporal expectation. Nat. Neurosci. 14, 246-251.

Jaramillo, S., Borges, K., and Zador, A.M. (2014). Auditory Thalamus and Auditory Cortex Are Equally Modulated by Context during Flexible Categorization of Sounds. J. Neurosci. 34, 5291-5301.

Jen, P.H.H., Chen, Q.C.C., and Sun, X.D.D. (1998). Corticofugal regulation of auditory sensitivity in the bat inferior colliculus. J. Comp. Physiol. A. 183, 683-697.

Jiang, D.-Y., Wu, Z., Forsyth, C.T., Hu, Y., Yee, S.-P., and Chen, G. (2018). GABAergic deficits and schizophrenia-like behaviors in a mouse model carrying patientderived neuroligin-2 R215H mutation. Mol. Brain 11, 31 .

Johnson, J.H., Turner, C.W., Zwislocki, J.J., and Margolis, R.H. (1993). Just noticeable differences for intensity and their relation to loudness. J. Acoust. Soc. Am. 93, 983-991.

Johnson, K.O., Hsiao, S.S., and Yoshioka, T. (2002). Review: Neural Coding and the Basic Law of Psychophysics. Neurosci. 8, 111-121. 
Kelly, J.B., Glenn, S.L., and Beaver, C.J. (1991). Sound frequency and binaural response properties of single neurons in rat inferior colliculus. Hear. Res. 56, 273-280.

Kilgard, M.P., and Merzenich, M.M. (1999). Distributed representation of spectral and temporal information in rat primary auditory cortex. Hear. Res. 134, 16-28.

Kim, J.J., and Jung, M.W. (2006). Neural circuits and mechanisms involved in Pavlovian fear conditioning: a critical review. Neurosci. Biobehav. Rev. 30, 188-202.

King, J., Insanally, M., Jin, M., Martins, A.R.O.R.O., D’amour, J.A., Froemke, R.C.C., D'amour, J.A., and Froemke, R.C.C. (2015). Rodent auditory perception: Critical band limitations and plasticity. Neuroscience 296, 55-65.

Kittel, M., Wagner, E., and Klump, G.M. (2002). An estimate of the auditory-filter bandwidth in the Mongolian gerbil. Hear. Res. 164, 69-76.

Klepper, A., and Herbert, H. (1991). Distribution and origin of noradrenergic and serotonergic fibers in the cochlear nucleus and inferior colliculus of the rat. Brain Res. 557, 190-201.

Klink, K.B., Dierker, H., Beutelmann, R., and Klump, G.M. (2010). Comodulation Masking Release Determined in the Mouse (Mus musculus) using a Flanking-band Paradigm. J. Assoc. Res. Otolaryngol. 11, 79-88.

Knolle, F., Schröger, E., and Kotz, S.A. (2013). Prediction errors in self- and externally-generated deviants. Biol. Psychol. 92, 410-416.

Kobrina, A., Toal, K.L., and Dent, M.L. (2018). Intensity difference limens in adult CBA/CaJ mice (Mus musculus). Behav. Processes 148, 46-48.

Kuo, R.I., and Wu, G.K. (2012). The Generation of Direction Selectivity in the Auditory System. Neuron 73, 1016-1027.

Kurt, S., and Ehret, G. (2010). Auditory discrimination learning and knowledge transfer in mice depends on task difficulty. Proc. Natl. Acad. Sci. U. S. A. 107, 8481-8485.

Laming, D. (2010). Fechner's law: where does the log transform come from? Seeing Perceiving 23, 155-171.

Langner, G. (1992). Periodicity coding in the auditory system. Hear. Res. 60, 115-142.

Langner, G., Sams, M., Heil, P., and Schulze, H. (1997). Frequency and periodicity are represented in orthogonal maps in the human auditory cortex: evidence from magnetoecephalography. J. Comp. Physiol. A. 181, 665-676.

Langner, G., Albert, M., and Briede, T. (2002). Temporal and spatial coding of periodicity information in the inferior colliculus of awake chinchilla (Chinchilla laniger). Hear. Res. 168, 110-130. 
Laufer, O., and Paz, R. (2012). Monetary Loss Alters Perceptual Thresholds and Compromises Future Decisions via Amygdala and Prefrontal Networks. J. Neurosci. 32, 6304-6311.

Laufer, O., Israeli, D., and Paz, R. (2016). Behavioral and Neural Mechanisms of Overgeneralization in Anxiety. Curr. Biol. 26, 713-722.

Leaver, A.M., and Rauschecker, J.P. (2010). Cortical Representation of Natural Complex Sounds: Effects of Acoustic Features and Auditory Object Category. J. Neurosci. 30, 7604-7612.

LeDoux, J.E. (2000). Emotion Circuits in the Brain. Annu. Rev. Neurosci. 23, 155-184.

LeDoux, J.E. (2014). Coming to terms with fear. Proc. Natl. Acad. Sci. 111, 2871-2878.

Li, W., Howard, J.D., Parrish, T.B., and Gottfried, J.A. (2008). Aversive learning enhances perceptual and cortical discrimination of indiscriminable odor cues. Science 319 , $1842-1845$.

Lissek, S., Rabin, S., Heller, R.E., Lukenbaugh, D., Geraci, M., Pine, D.S., and Grillon, C. (2010). Overgeneralization of Conditioned Fear as a Pathogenic Marker of Panic Disorder. Am. J. Psychiatry 167, 47-55.

Liu, E.H., Mercado, E., Church, B.A., Orduña, I., and Orduña, I. (2008). The easy-tohard effect in human (Homo sapiens) and rat (Rattus norvegicus) auditory identification. 122.

Ludwig, K.A., Miriani, R.M., Langhals, N.B., Joseph, M.D., Anderson, D.J., and Kipke, D.R. (2009). Using a Common Average Reference to Improve Cortical Neuron Recordings From Microelectrode Arrays. J. Neurophysiol. 101, 1679-1689.

Luiz, A., Lockmann, V., Afonso, F., Mourão, G., Flávio, M., and Moraes, D. (2017). Auditory fear conditioning modifies steady-state evoked potentials in the rat inferior colliculus. J Neurophysiol 118, 1012-1020.

Malmierca, M.S. (2004). The Inferior Colliculus: A Center for Convergence of Ascending and Descending Auditory Information. Neuroembryology Aging 3, 215-229.

Malmierca, M.S., Merchán, M.A., Henkel, C.K., and Oliver, D.L. (2002). Direct projections from cochlear nuclear complex to auditory thalamus in the rat. J. Neurosci. 22, 10891-10897.

Markov, N.T., and Kennedy, H. (2013). The importance of being hierarchical. Curr. Opin. Neurobiol. 23, 187-194.

Marsh, R.A., Fuzessery, Z.M., Grose, C.D., and Wenstrup, J.J. (2002). Projection to the inferior colliculus from the basal nucleus of the amygdala. J. Neurosci. 22, 10449-10460. 
McDermott, J.H., Schemitsch, M., and Simoncelli, E.P. (2013). Summary statistics in auditory perception. Nat. Neurosci. 16, 493-498.

McShefferty, D., Whitmer, W.M., and Akeroyd, M.A. (2015). The just-noticeable difference in speech-to-noise ratio. Trends Hear. 19.

Mercado, E., Orduña, I., and Nowak, J.M. (2005). Auditory Categorization of Complex Sounds by Rats (Rattus norvegicus). J. Comp. Psychol. 119, 90-98.

Miller, G.A. (1994). The magical number seven, plus or minus two: some limits on our capacity for processing information. 1956. Psychol. Rev. 101, 343-352.

Miller, J.L., Green, K.P., and Reeves, A. (1986). Speaking Rate and Segments: A Look at the Relation between Speech Production and Speech Perception for the Voicing Contrast. Phonetica 43, 106-115.

Miller, J.M., Sutton, D., Pfingst, B., Ryan, A., Beaton, R., and Gourevitch, G. (1972). Single cell activity in the auditory cortex of Rhesus monkeys: behavioral dependency. Science $177,449-451$.

Mitani, A., Shimokouchi, M., and Nomura, S. (1983). Effects of stimulation of the primary auditory cortex upon colliculogeniculate neurons in the inferior colliculus of the cat. Neurosci. Lett. 42, 185-189.

Moshitch, D., Las, L., Ulanovsky, N., Bar-Yosef, O., and Nelken, I. (2006). Responses of neurons in primary auditory cortex (A1) to pure tones in the halothane-anesthetized cat. J. Neurophysiol. 95, 3756-3769.

Nabelek, I., and Hirsh, I.J. (1969). On the Discrimination of Frequency Transitions. J. Acoust. Soc. Am. 45, 1510-1519.

Nahum, M., Nelken, I., and Ahissar, M. (2008). Low-Level Information and HighLevel Perception: The Case of Speech in Noise. PLoS Biol. 6, e126.

Nevue, A.A., Felix, R.A., and Portfors, C. V. (2016). Dopaminergic projections of the subparafascicular thalamic nucleus to the auditory brainstem. Hear. Res. 341, 202-209.

Newman, R.S., and Sawusch, J.R. (1996). Perceptual normalization for speaking rate: Effects of temporal distance. Percept. Psychophys. 58, 540-560.

Nieh, E.H., Kim, S.-Y., Namburi, P., and Tye, K.M. (2013). Optogenetic dissection of neural circuits underlying emotional valence and motivated behaviors. Brain Res. 1511, 73-92.

Niell, C.M., and Stryker, M.P. (2010). Modulation of Visual Responses by Behavioral State in Mouse Visual Cortex. Neuron 65, 472-479.

Ohl, F.W., Scheich, H., and Freeman, W.J. (2001). Change in pattern of ongoing 
cortical activity with auditory category learning. Nature 412, 733-736.

Ono, M., Bishop, D.C., and Oliver, D.L. (2017). Identified GABAergic and Glutamatergic Neurons in the Mouse Inferior Colliculus Share Similar Response Properties. J. Neurosci. 37, 8952-8964.

Ortiz, J.A., and Wright, B.A. (2009). Contributions of procedure and stimulus learning to early, rapid perceptual improvements. J. Exp. Psychol. Hum. Percept. Perform. 35, 188194.

Osmanski, M.S., and Wang, X. (2015). Behavioral Dependence of Auditory Cortical Responses. Brain Topogr. 28, 365-378.

Otazu, G.H., Tai, L.-H.H., Yang, Y., and Zador, A.M. (2009). Engaging in an auditory task suppresses responses in auditory cortex. Nat. Neurosci. 12, 646-654.

Parente, D.J., Garriga, C., Baskin, B., Douglas, G., Cho, M.T., Araujo, G.C., and Shinawi, M. (2017). Neuroligin 2 nonsense variant associated with anxiety, autism, intellectual disability, hyperphagia, and obesity. Am. J. Med. Genet. Part A 173, 213-216.

Peruzzi, D., Bartlett, E., Smith, P.H., and Oliver, D.L. (1997). A monosynaptic GABAergic input from the inferior colliculus to the medial geniculate body in rat. J. Neurosci. 17, 3766-3777.

Phillips, M.G., Lenzi, S.C., and Geerts, J.P. (2017). Cortical Predictive Mechanisms of Auditory Response Attenuation to Self-Generated Sounds. J. Neurosci. 37, 5393-5394.

Poli, E., and Angrilli, A. (2015). Greater general startle reflex is associated with greater anxiety levels: a correlational study on 111 young women. Front. Behav. Neurosci. 9, 10.

Polley, D.B. (2006). Perceptual Learning Directs Auditory Cortical Map Reorganization through Top-Down Influences. J. Neurosci. 26, 4970-4982.

Poremba, A., Jones, D., and Gonzalez-Lima, F. (1997). Metabolic Effects of Blocking Tone Conditioning on the Rat Auditory System. Neurobiol. Learn. Mem. 68, 154-171.

Potashner, S.J.J., Dymczyk, L., and Deangelis, M.M.M. (1988). D-aspartate uptake and release in the guinea pig spinal cord after partial ablation of the cerebral cortex. J. Neurochem. 50, 103-111.

Reed, A., Riley, J., Carraway, R., Carrasco, A., Perez, C., Jakkamsetti, V., and Kilgard, M.P. (2011). Cortical Map Plasticity Improves Learning but Is Not Necessary for Improved Performance. Neuron 70, 121-131.

Regan, D., and Beverley, K.I. (1985). Postadaptation orientation discrimination. J. Opt. Soc. Am. A. 2, 147-155.

Resnik, J., and Paz, R. (2014). Fear generalization in the primate amygdala. Nat. 
Neurosci. 18, 188-190.

Resnik, J., Sobel, N., and Paz, R. (2011). Auditory aversive learning increases discrimination thresholds. Nat. Neurosci. 14, 791-796.

Rodgers, C.C.C.C., and DeWeese, M.R.R.R. (2014). Neural Correlates of Task Switching in Prefrontal Cortex and Primary Auditory Cortex in a Novel Stimulus Selection Task for Rodents Chris. Neuron 82, 1157-1170.

Rohrbaugh, M., Brennan, J.F., and Riccio, D.C. (1971). Control of two-way shuttle avoidance in rats by auditory frequency and intensity. J. Comp. Physiol. Psychol. 75, 324330.

Rummell, B.P., Klee, J.L., and Sigurdsson, T. (2016). Attenuation of Responses to Self-Generated Sounds in Auditory Cortical Neurons. J. Neurosci. 36, 12010-12026.

Rutkowski, R.G., and Weinberger, N.M. (2005). Encoding of learned importance of sound by magnitude of representational area in primary auditory cortex. Proc. Natl. Acad. Sci. U. S. A. 102, 13664-13669.

Rybalko, N., Šuta, D., Nwabueze-Ogbo, F., and Syka, J. (2006). Effect of auditory cortex lesions on the discrimination of frequency-modulated tones in rats. Eur. J. Neurosci. $23,1614-1622$.

Sagi, D. (2011). Perceptual learning in Vision Research. Vision Res. 51, 1552-1566.

Salzman, C.D., and Fusi, S. (2010). Emotion, Cognition, and Mental State Representation in Amygdala and Prefrontal Cortex. Annu. Rev. Neurosci. 33, 173-202.

Sato, A. (2008). Action observation modulates auditory perception of the consequence of others' actions. Conscious. Cogn. 17, 1219-1227.

Schechtman, E., Laufer, O., and Paz, R. (2010). Negative Valence Widens Generalization of Learning. J. Neurosci. 30, 10460-10464.

Scheich, H., Brechmann, A., Brosch, M., Budinger, E., Ohl, F.W., Selezneva, E., Stark, H., Tischmeyer, W., and Wetzel, W. (2011). Behavioral semantics of learning and crossmodal processing in auditory cortex: The semantic processor concept. Hear. Res. 271, $3-15$.

Schneider, D.M., Nelson, A., and Mooney, R. (2014). A synaptic and circuit basis for corollary discharge in the auditory cortex. Nature 513, 189-194.

Schneider, D.M., Sundararajan, J., and Mooney, R. (2018). A cortical filter that learns to suppress the acoustic consequences of movement. Nature 561, 391-395.

Schofield, B.R. (2010). Projections from auditory cortex to midbrain cholinergic neurons that project to the inferior colliculus. Neuroscience 166, 231-240. 
Schouten, M.E.H. (1985). Identification and discrimination of sweep tones. Percept. Psychophys. 37, 369-376.

Schreiner, C.E., and Langner, G. (1988). Periodicity coding in the inferior colliculus of the cat. II. Topographical organization. J. Neurophysiol. 60, 1823-1840.

Schreiner, C.E., and Langner, G. (1997). Laminar fine structure of frequency organization in auditory midbrain. Nature 388, 383-386.

Schreiner, C.E., Read, H.L., and Sutter, M.L. (2000). Modular Organization of Frequency Integration in Primary Auditory Cortex. Annu. Rev. Neurosci. 23, 501-529.

Schröger, E., Marzecová, A., and SanMiguel, I. (2015). Attention and prediction in human audition: a lesson from cognitive psychophysiology. Eur. J. Neurosci. 41, 641-664.

Schulze, H., and Scheich, H. (1999). Discrimination learning of amplitude modulated tones in Mongolian gerbils. Neurosci. Lett. 261, 13-16.

Schulze, H., Hess, A., Ohl, F.W., and Scheich, H. (2002). Superposition of horseshoelike periodicity and linear tonotopic maps in auditory cortex of the Mongolian gerbil. Eur. J. Neurosci. 15, 1077-1084.

Scott, B.H., Malone, B.J., and Semple, M.N. (2007). Effect of behavioral context on representation of a spatial cue in core auditory cortex of awake macaques. J. Neurosci. 27, 6489-6499.

Seger, C.A., and Peterson, E.J. (2013). Categorization=decision making+generalization. Neurosci. Biobehav. Rev. 37, 1487-1200.

Shalev, L., Paz, R., and Avidan, G. (2018). Visual Aversive Learning Compromises Sensory Discrimination. J. Neurosci. 38, 2766-2779.

Shepard, R.N. (1987). Toward a universal law of generalization for psychological science. Science 237, 1317-1323.

Shepard, R.N. (1991). Integrality versus separability of stimulus dimensions: From an early convergence of evidence to a proposed theoretical basis. Percept. Struct. Essays Honor Wendell R. Garner 53-71.

Shibata, K., Sagi, D., and Watanabe, T. (2014). Two-stage model in perceptual learning: toward a unified theory. Ann. N. Y. Acad. Sci. 1316, 18-28.

Shimaoka, D., Harris, K.D., and Carandini, M. (2018). Effects of Arousal on Mouse Sensory Cortex Depend on Modality. Cell Rep. 22, 3160-3167.

Shuler, M.G., and Bear, M.F. (2006). Reward timing in the primary visual cortex. Science $311,1606-1609$.

Singla, S., Dempsey, C., Warren, R., Enikolopov, A.G., and Sawtell, N.B. (2017). A 
cerebellum-like circuit in the auditory system cancels responses to self-generated sounds. Nat. Neurosci. 20, 943-950.

Skottun, B.C., Bradley, A., Sclar, G., Ohzawa, I., and Freeman, R.D. (1987). The effects of contrast on visual orientation and spatial frequency discrimination: a comparison of single cells and behavior. J. Neurophysiol. 57, 773-786.

Skottun, B.C., Shackleton, T.M., Arnott, R.H., and Palmer, A.R. (2001). The ability of inferior colliculus neurons to signal differences in interaural delay. Proc. Natl. Acad. Sci. U. S. A. $98,14050-14054$.

Slee, S.J., and David, S. V (2015). Rapid Task-Related Plasticity of Spectrotemporal Receptive Fields in the Auditory Midbrain. J. Neurosci. 35, 13090-13102.

Smith, N.A., and Schmuckler, M.A. (2008). Dial A440 for absolute pitch: Absolute pitch memory by non-absolute pitch possessors. J. Acoust. Soc. Am. 123, EL77-EL84.

Smith, J.D., Zakrzewski, A.C., Johnson, J.M., Valleau, J.C., and Church, B.A. (2016). Categorization: The View from Animal Cognition. Behav. Sci. (Basel, Switzerland) 6, 12.

Soto, F.A., and Wasserman, E.A. (2010). Integrality/separability of stimulus dimensions and multidimensional generalization in pigeons. J. Exp. Psychol. Anim. Behav. Process. 36, 194-205.

Soto, F.A., Gershman, S.J., and Niv, Y. (2014). Explaining compound generalization in associative and causal learning through rational principles of dimensional generalization. Psychol. Rev. 121, 526-558.

Spiering, B.J., and Ashby, F.G. (2008). Initial training with difficult items facilitates information integration, but not rule-based category learning. Psychol. Sci. 19, 1169-1177.

Stone, H., and Bosley, J.J. (1965). Olfactory Discrimination and Weber's Law. Percept. Mot. Skills 20, 657-665.

Suga, N. (2012). Tuning shifts of the auditory system by corticocortical and corticofugal projections and conditioning. 36.

Suga, N., and Ma, X. (2003). Multiparametric corticofugal modulation and plasticity in the auditory system. Nat. Rev. Neurosci. 4, 783-794.

Suga, N., and Shimozawa, T. (1974). Site of neural attenuation of responses to selfvocalized sounds in echolocating bats. Science 183, 1211-1213.

Suga, N., Gao, E., Zhang, Y., Ma, X., and Olsen, J.F.F. (2000). The corticofugal system for hearing: recent progress. Proc. Natl. Acad. Sci. U. S. A. 97, 11807-11814.

Suga, N., Xiao, Z., Ma, X., and Ji, W. (2002). Plasticity and corticofugal modulation for hearing in adult animals. Neuron 36, 9-18. 
Sun, X., Chen, Q.C.C., and Jen, P.H.H. (1996). Corticofugal control of central auditory sensitivity in the big brown bat, Eptesicus fuscus. Neurosci. Lett. 212, 131-134.

Syka, J., Rybalko, N., Brožek, G., and Jilek, M. (1996). Auditory frequency and intensity discrimination in pigmented rats. Hear. Res. 100, 107-113.

Taberner, A.M., and Liberman, M.C. (2005). Response Properties of Single Auditory Nerve Fibers in the Mouse. J. Neurophysiol. 93, 557-569.

Talwar, S.K., and Gerstein, G.L. (1998). Auditory frequency discrimination in the white rat. Hear. Res. 126, 135-150.

Terman, M. (1970). Discrimination of auditory intensities by rats1. J. Exp. Anal. Behav. $13,1333756$.

Townsend, G., Peloquin, P., Kloosterman, F., Hetke, J.F., and Leung, L.S. (2002). Recording and marking with silicon multichannel electrodes. Brain Res. Brain Res. Protoc. 9, 122-129.

Varoqueaux, F., Jamain, S., and Brose, N. (2004). Neuroligin 2 is exclusively localized to inhibitory synapses. Eur. J. Cell Biol. 83, 449-456.

Varoqueaux, F., Aramuni, G., Rawson, R.L., Mohrmann, R., Missler, M., Gottmann, K., Zhang, W., Südhof, T.C., and Brose, N. (2006). Neuroligins Determine Synapse Maturation and Function. Neuron 51, 741-754.

Verzijden, M.N., Etman, E., van Heijningen, C., van der Linden, M., and ten Cate, C. (2007). Song discrimination learning in zebra finches induces highly divergent responses to novel songs. Proc. R. Soc. B Biol. Sci. 274, 295-301.

Vinck, M., Batista-Brito, R., Knoblich, U., and Cardin, J.A.A. (2015). Arousal and Locomotion Make Distinct Contributions to Cortical Activity Patterns and Visual Encoding. Neuron 86, 740-754.

Wang, L., Gillis-Smith, S., Peng, Y., Zhang, J., Chen, X., Salzman, C.D., Ryba, N.J.P., and Zuker, C.S. (2018). The coding of valence and identity in the mammalian taste system. Nature 558, 127-131.

Warren, J.M. (1954). Perceptual dominance in discrimination learning by monkeys. J. Comp. Physiol. Psychol. 47, 290-292.

Wasserman, E.A., and Miller, R.R. (1997). WHAT'S ELEMENTARY ABOUT ASSOCIATIVE LEARNING? Annu. Rev. Psychol. 48, 573-607.

Watanabe, T., and Sasaki, Y. (2015). Perceptual Learning: Toward a Comprehensive Theory. Annu. Rev. Psychol. 66, 197-221.

Watanabe, T., Náñez, J.E., and Sasaki, Y. (2001). Perceptual learning without 
perception. Nature 413, 844-848.

Weinberger, N.M. (2004). Specific long-term memory traces in primary auditory cortex. Nat. Rev. Neurosci. 5, 279-290.

Weinberger, N.M. (2007). Associative representational plasticity in the auditory cortex: A synthesis of two disciplines. Learn. Mem. 14, 1-16.

Westheimer, G., Shimamura, K., and McKee, S.P. (1976). Interference with lineorientation sensitivity. J. Opt. Soc. Am. 66, 332-338.

Wetzel, W., Wagner, T., Ohl, F.W., and Scheich, H. (1998). Categorical discrimination of direction in frequency-modulated tones by Mongolian gerbils. Behav. Brain Res. 91, 29-39.

Winer, J.A. (2006). Decoding the auditory corticofugal systems. Hear. Res. 212, 1-8.

Winer, J.A., and Schreiner, C.E. (2005). The Inferior Colliculus.

$\mathrm{Wu}$, Y., and Yan, J. (2007). Modulation of the receptive fields of midbrain neurons elicited by thalamic electrical stimulation through corticofugal feedback. J. Neurosci. 27, 10651-10658.

Xu, S., Jiang, W., Poo, M.-M., and Dan, Y. (2012). Activity recall in a visual cortical ensemble. Nat. Neurosci. 15, 449-455, S1-2.

Yan, J., and Ehret, G. (2001). Corticofugal reorganization of the midbrain tonotopic map in mice. Neuroreport 12, 3313-3316.

Yan, J., and Ehret, G. (2002). Corticofugal modulation of midbrain sound processing in the house mouse. Eur. J. Neurosci. 16, 119-128.

Zanker, J.M. (1995). Does Motion Perception Follow Weber's Law? Perception 24, $363-372$.

Zhang, L.I., Tan, A.Y.Y., Schreiner, C.E., and Merzenich, M.M. (2003). Topography and synaptic shaping of direction selectivity in primary auditory cortex. Nature 424, 201205.

Zhang, Q., Liu, H., McGee, J., Walsh, E.J., Soukup, G.A., and He, D.Z.Z. (2013). Identifying MicroRNAs Involved in Degeneration of the Organ of Corti during AgeRelated Hearing Loss. PLoS One 8, e62786.

Zhang, Y., Suga, N., and Yan, J. (1997). Corticofugal modulation of frequency processing in bat auditory system. Nature 387, 900-903. 


\section{ACKNOWLEDGMENTS}

To begin with I would like to thank my supervisor Dr. Livia de Hoz, for having me in the lab and all the support, guidance and encouragement through the years. Thank you for always being there whenever needed, and being such a kind, patient, and great supervisor.

I would like to thank Prof. Nave for the opportunity to work in such a great environment and support on my work.

I would like to thank Prof. Gütig for the valuable advices and financial support. I would also like to thank the members of my thesis committee: Prof. Gollisch and Prof. Gail, thank you for the fruitful feedback during discussions and guide throughout the project.

I want to thank Dilja Krueger-Burg for sharing the Neuroligin 2 mice and the interesting discussions; To Iva Tzvetanova for the help with the confocal microscope; to Burak Bali for his help with the behavioral experiments; to Harry Scherer for all the technical support; to the Neurogenetics department for creating such a nice environment; to all the stuff members in the MPI-em for providing numerous support.

I would like to thank all the members of the Lab: Sharlen Moore, Swati Subramanian, Philipp van Kronenberg, Linus Milinski and Hugo Cruces, for the scientific and nonscientific discussions, the amazing international food, and making such a nice atmosphere in the lab.

A special thank to Micheal Hörner, Sandra Drube and the IMPRS Neuroscience program for all the assistance and counselling.

I would like to thank all my friends in Göttingen, especially the class 2012/13 of the neuroscience program, for making the experience here so great and unforgettable.

Last, but not least, I would like to thank my family. Thank you, baba and mama, for the unconditional love and being always supportive. I also thank mammy, daddy, my cousin and my aunts for the care, love and support. Grandma, thank you for loving me and always care my life. I hope I made you proud. Stella, my daughter, thank you for bringing so much joy, love and happiness in my life. I would like to thank my husband, Xueqiu, for everything, I will not achieve what I have now without you. 


\section{List of abbreviations}

\begin{tabular}{|c|c|}
\hline A1 & primary auditory cortex \\
\hline $\mathbf{A C}$ & auditory cortex \\
\hline $\mathbf{A M}$ & amplitude modulation \\
\hline ASR & acoustic startle response \\
\hline BF & best frequency \\
\hline CNIC & central nucleus of the inferior colliculus \\
\hline CS & conditioned stimulus \\
\hline DCIC & dorsal cortex of the inferior colliculus \\
\hline ECIC & external cortex of the inferior colliculus \\
\hline FAR & false alarm rate \\
\hline FM & frequency modulation \\
\hline GABAergic & gamma-aminobutyric acid-ergic \\
\hline IC & inferior colliculus \\
\hline JNDs & just-noticeable-differences \\
\hline KO & knockout \\
\hline LFP & local field potential \\
\hline LTP & long-term potentiation \\
\hline MGB & medial geniculate body \\
\hline MUA & multiunit activity \\
\hline Nlgn2 & neuroligin 2 \\
\hline PPI & pre-pulse inhibition \\
\hline PSTH & peri-stimulus time histogram \\
\hline $\mathbf{R F}$ & receptive field \\
\hline SNR & signal-to-noise ratia \\
\hline SOC & superior olivary complex \\
\hline STRF & spectro-temporal receptive field \\
\hline TRF & tonal receptive field \\
\hline VTA & ventral tegmental area \\
\hline
\end{tabular}




\section{List of figures}

Figure 0-1 Absolute-judgement based discrimination protocol and Audiobox apparatus. . 13 Figure 0-2 Acoustic startle apparatus for frequency discrimination acuity measurement. . 19 Figure $0-3$ Individually moveable tetrodes implantation ................................................ 22

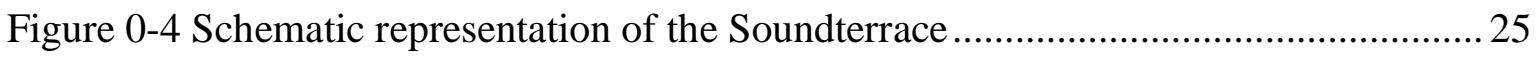

Figure 0-5 Auditory discrimination task in the Soundterrace ........................................26

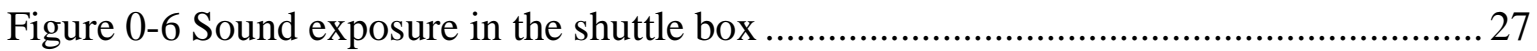

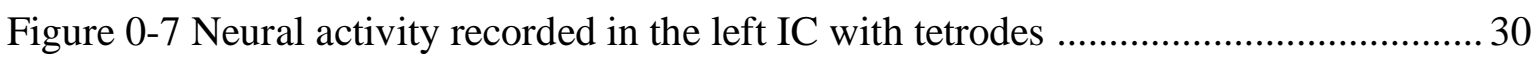

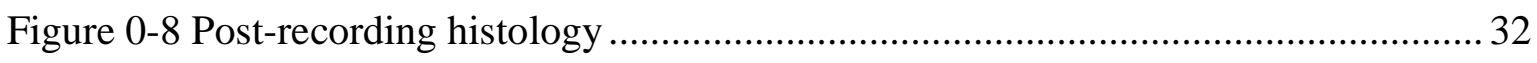

Figure 0-9 Schematic representation of recording with silicon probe in the left IC ........... 34

Figure 1-1 Discrimination training and generalization testing in the Audiobox ................. 40

Figure 1-2 Individual performance and visit length analysis .......................................... 42

Figure 1-3 Discrimination performance deteriorated as the safe-to-conditioned $\Delta \mathrm{F}$

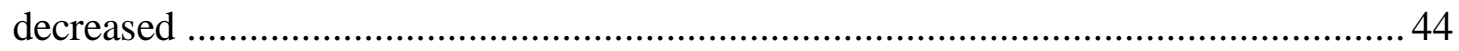

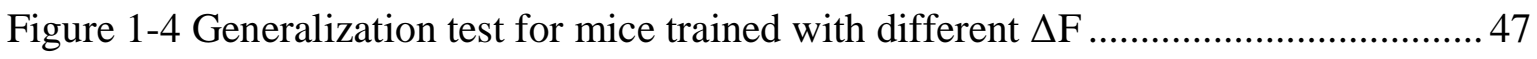

Figure 1-5 Asymmetrical generalization gradients in mice trained with small $\Delta \mathrm{Fs}$.......... 48

Figure 1-6 Neuroligin 2 knockout mice showed impaired discrimination but normal

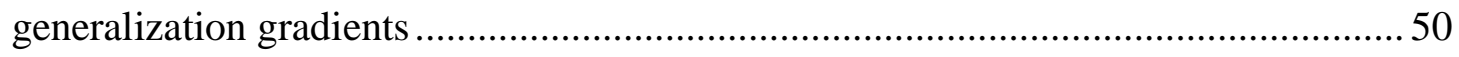

Figure 1-7 The direction of conditioning along the frequency axis affected learning, but not

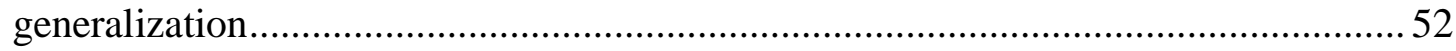

Figure 1-8 Previous task experience did not facilitate discrimination but shifted generalization gradients . .53

Figure 1-9 Retraining to another conditioned tone shifted psychometric threshold ...........56

Figure 1-10 Audiobox learning led to $\Delta \mathrm{F}$-specific increase in discrimination acuity around the conditioned tone 58

Figure 2-1 Generalization of FMs was along the frequency dimension, but not the direction

Figure 2-2 Multidimensional generalization of FMs. .73

Figure 2-3 Mice can be trained to do unidimensional categorization. ............................... 75

Figure 2-4 Generalization along the non-preferred dimension was localized.................... 77

Figure 2-5 Bidirectional generalization of periodic sound envelopes .............................. 78

Figure 2-6 Learning curves and discrimination comparison ........................................ 81

Figure 2-7 Learning curves for discrimination of stimuli that differed in 2 dimensions .... 83 
Figure 3-1 Pure-tone discrimination training in the Soundterrace .................................. 91

Figure 3-2 Task engagement increased spontaneous activity in IC ................................ 93

Figure 3-3 Spontaneous activity was not changed during exposure phase ....................... 95

Figure 3-4 task engagement selectively suppressed sound-evoked activity.......................96

Figure 3-5 Increase in global gain and signal-to-noise ratio induced by engaging in a

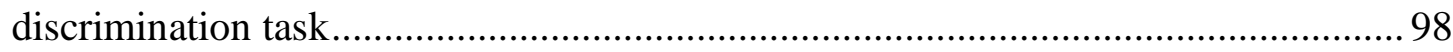

Figure 3-6 Lack of changes in global gain evoked by discrimination training .................. 99

Figure 3-7 Lack of changes in global gain evoked by acoustic stimuli associated with reward 100

Figure 4-1 Sound exposure in the Audiobox 108

Figure 4-2 Plasticity induced by sound exposure was independent of water association . 111

Figure 4-3 The effect of sound exposure was independent of water association

Figure 4-4 Unpredictable sound exposure did not elicit plasticity in the inferior colliculus

Figure 4-5 Movement-related sound exposure elicited plasticity in IC that was comparable to that of normal exposure 118

Figure 4-6 Predictable sound exposure elicited a global suprathreshold shift in BF 119

Figure 4-7 Sound exposure of FMs did not elicit change in response gain in IC of freely behaving mouse

Figure 4-8 Self-initiation decreased ongoing local field potential in the inferior colliculus 


\section{List of tables}

Table 0-1 Acoustic stimuli used for sound exposure in the shuttle box.

29 
Herewith I declare, that I prepared the PhD Thesis "Auditory associative learning and its neural correlates in the auditory midbrain" on my own and with no other sources and aids than quoted.

Göttingen, 29.11.2018

Chi Chen 